\title{
A FRAMEWORK FOR LinkED DATA QuaLity BASED ON \\ Data Profiling and RDF Shape INDUCTION
}

Author: NANDAna Mihindukulasooriya

Supervisor: Dr. Raúl García Castro

Supervisor: Prof. Dr. Asunción Gómez Pérez

February 2020 



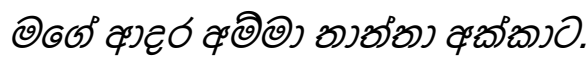
A Miriam. 

There are no small problems. Problems that appear small are large problems that are not understood. Santiago Ramón y Cajal, Advice for a Young Investigator, 1897 


\section{Acknowledgements}

Finally, the day has come to write the acknowledgements, the last page to write before I deposit the thesis. Now is the time to take a deep breath and remember everyone who has helped me during this journey. It was not an easy road, especially with the unexpected health issues of both my parents. I would not have made it without the help of many kind people who went out of their way to help me get to this goal.

First and foremost, I would like to thank my parents, without whose tireless efforts and dedication I would have never made this far. Their love, heartfelt blessings, and encouragement kept me going during the most challenging moments. Also, I want to thank my sister, the best sister in the world, who is giving my parents the care and camaraderie they deserve while I was away. Tambien quiero agradecerle a Miriam, Luciano, Maria, Vanesa y Pedro, mi familia en España, por su apoyo y cariño.

Muchísimas gracias Asun por todo tu apoyo, ayudas y consejos y por confíar en mi. Desde mi primer día en OEG, me has ayudado con todo, no solo académicamente, sino siempre que tuve problemas con cosas burocráticas. Igualmente quiero agradecerle a Raul que me ha enseñado a como hacer las cosas bien tanto en la investigación como en la tesis. De ti, he aprendido un montón. Gracias Oscar por todas tus ayudas y consejos para seguir en este mundo de web semántica y también por presentarme a mucha gente del area. Muchas gracias Mariano y Víctor por todas nuestras colaboraciones juntos, vuestra amistad y por ayudarme siempre en cualquir cosa que necesitaba.

Estuve siempre con mucho gusto en el equipo de $4 \mathrm{~V}$, con quien más trabajé durante los últimos días de la tesis. Gracias Víctor, Jorge, Elena y tambien a Nieves, Jesus, Arantza, Eduardo, Sergio, Raquel, Iker, Alfredo y otros por muchos recuerdos inolvidables de este proyecto, reuniones, viajes, cenas.

Quiero también darle las gracias a mis compañeros de OEG por los buenos tiempos que pase en España. Siempre disfruté mucho de nuestras discusiones de comida con Mariano, Freddy, Raul, Ahmed, Andrea, Lupe, Elena, Olga, Maria y todos los que subian a CAIT. Siempre han surgido muchas "crazy idea" allí como TourismKG. También gracias al equipo de hackatones con Carlos, Esteban, Fernando; fué un auténtico placer trabajar con vosotros.

Muchísimas gracias Ana, Jose Angel, Victoria y Almudena por ayudarme con las 
tareas administrativas. También Raúl Alcázar por tu ayuda con los temas técnicos. Gracias Idafen por los logos.

Quiero agradecer a todos compañeros y ex-compañeros, Alba, Alejandro Carrera, Alejandro Llaves, Alexander Garcia, Almudena, Alvaro, Andres, Angelito, Boris, Dani Vila, Daniel Garijo, David Chaves, Diego Collarana, Edna, Elvira, Emilio, Francisco, Francisco Martin, Francisco Siles, Ghis, Idafen, Irlan, Javier Bajo, JeanPaul, José Luis, Jose Mora, Juan, Julia, Kartik, Luis Vilches, Lupe, Mari-Carmen, Maria Poveda, María Navas, María Pérez, Mariano Fernández, Miguel Ángel, Miguel Esteban, Nelson, Olga, Pablo, Paco Yedro, Paola, Patricia, Pedro del Pozo, Pierre, Rafa, Riccardo, Rosangelis, Samuel, Socorro, Vicky, Víctor y otros.

I would like to give special thanks to Rifat, Filip, Giuseppe, Mariano, Dimitris, Maria, Jeremy with whom I got the opportunity to have productive collaborations. Thank you, Giuseppe and Oshani, for your valuable feedback on the thesis.

My internship at IBM Research was a turning point in my research career. I would like to thank Oktie, Alfio, Sarthak, Micheal, Gaetano, Nicolas, Faisal, and Mariano Rodriguez for your friendship and helping me out to make the research stay as productive as possible. It only took three months for me to fall in love with the Knowledge Induction Team and the interesting challenges solved by the group.

During the thesis, I got the great opportunity to work with the W3C community. I would like to thank Phil Archer, Sandro, Andrei, Eric from W3C, Arnaud, Alexandre, Ashok, Bart, Cody, David Wood, Henry, John, Kingsley, Olivier, Roger, Ruben, Serena, Sergio, Steve Speicher, and Ted from Linked Data Platform WG, and Amrapali, Antoine, Jeremy, and Riccardo from Data on the Web Best Practices WG for the interesting discussions and collaborations.

I can not forget my friends in Sri Lanka, Ratiyala, Chamara, Tharindu, Sujith, Pras and many others, who were there whenever I needed any help. Studying abroad, I was at ease knowing that I can always rely on you in case I needed help. Also special mention to my cousins Janaka ayya, Duminda ayya, Anura ayya and others for helping my parents whenever they needed something.

Ahora me doy cuenta que es difícil poner nombres porque siempre se te olvida alguien, pero quiero dar las gracias a todas las personas que de una forma u otra me han ayudado con las pequeñas y grandes cosas en este camino.

Por último, quiero agradecer a quien más me ha soportado en este largo viaje y siempre ha estado a mi lado hasta el final. Muchísimas gracias Miriam por tu cariño, paciencia y comprensión, por apoyarme siempre tanto en los momentos buenos como en los malos. Gracias por ser tan buena persona. Sin ti todo esto hubiera sido más difícil.

The work in this thesis has been supported by the FPI grant (BES-2014-068449) under the project $4 \mathrm{~V}$ of the Spanish Ministry of Education, Culture and Sport. I thank the ministry for their generous support. 


\section{Abstract}

In the era of digital transformation, where most decision-making and artificial intelligence (AI) applications are becoming data-driven, data is becoming an essential asset. Linked Data, published in structured, machine-readable formats, with explicit semantics using Semantic Web standards, and with links to other data, is even more useful. The Linked (Open) Data cloud is growing with millions of new triples each year. Nevertheless, as we discuss in this thesis, such vast amounts of data bring several new challenges in ensuring the quality of Linked Data.

The main goal of this thesis is to propose novel and scalable methods for automatic quality assessment and repair of Linked Data. The motivation for it is to significantly reduce the manual effort required by current quality assessment and repair, and to propose novel methods suitable for large-scale Linked Data sources such as DBpedia or Wikidata. The main hypothesis of this work is that data profiling metrics and automatic $R D F$ Shape induction can be used to develop scalable and automatic quality assessment and repair methods.

In this context, the following main contributions are delivered in this thesis:

- $L D Q M$, a Linked Data Quality Model for representing Linked Data quality in a standard manner and LD Sniffer, a tool based on LDQM for validating accessibility of Linked Data. LDQM contains 15 quality characteristics, 89 base measures, 23 derived measures, and 124 quality indicators.

- Loupe, a framework for Linked Data profiling that includes the Loupe Extended Dataset Description Model and a suite of Linked Data profiling tools. The model consists of 84 Linked Data profiling metrics useful for quality assessment and repair tasks. Loupe tools have been used to evaluate 26 thousand datasets containing 34 billions of triples and Loupe contributed to the winning system of ISWC Semantic Web Challenge 2017. The Loupe Web portal has been visited more than 40,000 times by $\sim 3000$ unique visitors from 87 countries.

- An automatic RDF Shape induction method that follows a data-driven approach to induce integrity constraints using data profiling metrics as features. The proposed method achieved an $\mathrm{F} 1$ of $98.81 \%$ in deriving maximum cardinality 
constraints, an $\mathrm{F} 1$ of $97.30 \%$ in deriving minimum cardinality constraints, and an $\mathrm{F} 1$ of $95.94 \%$ in deriving range constraints.

- Four methods for automatic quality assessment and repair using RDF Shapes and data profiling metrics. They are motivated by several practical use cases that cover both Linked Data generation process and output and also cover both public and enterprise data. The four methods include (a) a method for detecting inconsistent mappings, (b) a method for detecting and eliminating noisy triples produced by open information extraction tools, (c) a method to repair links in RDF data, and (d) a method to complete type information in Linked Data. Each method demonstrates a high performance ( $\sim 90 \%$ and above) in their respective tasks.

Several research projects, such as $4 \mathrm{~V}$, 3Cixty, BNE, and MappingPedia have already exploited the contributions of the thesis. In conclusion, we show that Linked Data research problems can learn from older paradigms, such as relational data. Through validating nine hypotheses related to the objectives of this thesis, we demonstrate that data profiling metrics can be used to develop scalable automatic methods for Linked Data quality assessment and repair with high accuracy. 


\section{Resumen}

En la era de la transformación digital, donde la mayoría de las aplicaciones de toma de decisiones e inteligencia artificial (IA) están siendo impulsadas por los datos, los datos se han convertido en un recurso esencial. Linked Data (los datos enlazados), publicados en formatos estructurados y legibles por máquinas, con una semántica explícita que utiliza estándares web semánticos y con enlaces a otros datos, hacen que los datos sean aún más útiles. La nube de los datos enlazados está creciendo, con millones de tripletas nuevas cada año. Sin embargo, como discutimos en esta tesis, esta enormes cantidad de datos trae consigo varios retos nuevos para garantizar la calidad de los datos enlazados.

El objetivo principal de esta tesis es proponer métodos novedosos y escalables para la evaluación automática de la calidad y la reparación de los datos enlazados. La motivación de este trabajo es reducir significativamente el esfuerzo manual requerido actualmente en las tareas de evaluación y reparación, y proponer nuevos métodos adecuados para fuentes a gran escala de los datos enlazados que contienen millones de tripletas como DBpedia o Wikidata.

La hipótesis principal de este trabajo es que las métricas de data profiling (perfiles de datos) y la inducción automática de $R D F$ Shapes puede utilizarse para desarrollar métodos escalables y automáticos de evaluación y reparación de calidad de los datos. Las siguientes contribuciones se presentan en esta tesis:

- $L D Q M$, un modelo para representar la calidad de los datos enlazados de manera estándar y LD Sniffer, una herramienta basada en LDQM que valida la accesibilidad de los datos enlazados. LDQM contiene 15 características de calidad, 89 medidas básicas, 23 medidas derivadas y 124 indicadores de calidad.

- Loupe, un framework para la creación de perfiles de los datos enlazados que incluye un modelo extendido de descripción de conjuntos de datos enlazados y un grupo de herramientas de creación de perfiles de los datos enlazados. Este modelo tiene 84 métricas de perfiles de los datos enlazados útiles para la evaluación y reparación de la calidad de los datos. Loupe se ha utilizado para evaluar 26 mil conjuntos de datos que contienen 34 mil millones de tripletas y ha contribuido al sistema ganador en el ISWC Semantic Web Challenge 2017. 
El portal web de Loupe ha sido visitado más de 40.000 veces por más de 3.000 visitantes de 87 países.

- Un método de inducción automático de RDF Shapes que sigue un enfoque basado en datos para inducir restricciones de integridad para datos RDF utilizando el perfil de datos. El método propuesto logró un F1 de 98.81\% en derivar restricciones de cardinalidad máxima, un F1 de $97.30 \%$ en derivar restricciones de cardinalidad mínima y un F1 de $95.94 \%$ en derivar restricciones de rango.

- Cuatro métodos para la evaluación y reparación automática de la calidad utilizando $R D F$ Shapes y métricas de perfiles de datos. Estos métodos fueron motivados por varios casos de uso práctico que cubren tanto el proceso de generación de datos enlazados, así como por los resultados con datos públicos y empresariales. Los cuatro métodos incluyen (a) un método para detectar mapeos inconsistentes, (b) un método para detectar y eliminar tripletas ruidosas extraídas por herramientas abiertas de extracción de información, (c) un método para reparar enlaces en datos RDF, y (d) Un método para completar la información de tipo en datos enlazados. Cada método demuestra un alto rendimiento ( $\sim 90 \%$ y superior) en sus respectivas tareas.

Varios proyectos de investigación como 4V, 3Cixty, BNE y MappingPedia ya han explotado las contribuciones de esta tesis. En conclusión, mostramos que la investigación de los datos enlazados puede aprender de paradigmas más antiguos como los datos relacionales. Mediante la validación de nueve hipótesis relacionadas con los objetivos de esta tesis, demostramos que las métricas de perfilado de datos pueden utilizarse para desarrollar métodos automáticos escalablesde de gran precisión para la evaluación y reparación de la calidad de Linked Data. 


\section{Contents}

Acknowledgements $\quad$ i

Abstract $\quad$ iii

$\begin{array}{lll}\text { Resumen } & \text { V }\end{array}$

List of Figures $\quad$ xi

List of Tables $\quad$ xiii

1 Introduction $\quad 1$

1.1 Linked Data Quality Lifecycle . . . . . . . . . . . . . . . . 6

1.2 Linked Data Quality Model . . . . . . . . . . . . . . . . . . 10

1.3 Data Profiling . . . . . . . . . . . . . . . . . . . . . . . . . . . . . . . . 11

1.4 RDF Shape Induction . . . . . . . . . . . . . . . . . . . 12

1.5 Methods for Quality Assessment and Repair . . . . . . . . . . . . . . 15

1.6 Main Contributions . . . . . . . . . . . . . . . . . . . 16

1.7 Derived Publications . . . . . . . . . . . . . . . 17

1.8 Registered Software . . . . . . . . . . . . . . . . . . . 21

1.9 Awards . . . . . . . . . . . . . . . . . . . . . . . . . . . . . . . . . . . . 22

1.10 Research Stays . . . . . . . . . . . . . . . . . . . . . 22

1.11 Work in Research and Innovation Projects and Standardization Bodies 23

1.12 Structure of the Document . . . . . . . . . . . . . . 25

2 State of the Art $\quad 26$

2.1 Linked Data Quality Model . . . . . . . . . . . . . . . 26

2.2 Linked Data Profiling . . . . . . . . . . . . . . . . . . . . . . . 33

2.3 RDF Shape Induction . . . . . . . . . . . . . . . . . 34

2.4 Methods for Linked Data Quality Assessment and Repair . . . . . . . 36

2.4.1 Linked Data Quality Assessment Tools . . . . . . . . . . . . . 36

2.4.2 Linked Data Repair Tools . . . . . . . . . . . . . . . 39 
2.5 Conclusions . . . . . . . . . . . . . . . . . . . . . 40

3 Work Objectives 42

3.1 Thesis Objectives . . . . . . . . . . . . . . . . . . . . . . 42

3.2 Open Research Problems . . . . . . . . . . . . . . . . . . . . 44

3.3 Contributions to the State of the Art . . . . . . . . . . . 48

3.4 Restrictions, Assumptions, and Hypotheses . . . . . . . . . . . 52

3.5 Evaluation Plan . . . . . . . . . . . . . . . . . . . . . 55

3.6 Research Methodology . . . . . . . . . . . . . . . . . . . 57

3.6.1 Methodological and Technological Inputs . . . . . . . . . 57

3.6.2 Description of the Research Process . . . . . . . . . . . . . 60

4 Linked Data Quality Model $\quad 62$

4.1 Preliminaries . . . . . . . . . . . . . . . . . . . 63

4.1 .1 Quality Models . . . . . . . . . . . . . . . . . . 63

4.1.2 Linked Data Quality Specification . . . . . . . . . . . 66

4.1.3 Existing Quality Specification and Assessment Ontologies . . . 67

4.2 Quality Model for Linked Data . . . . . . . . . . . . . . . . . 68

4.2.1 Identification of Base Measures . . . . . . . . . . . . 72

4.2 .2 Identification of Indicators . . . . . . . . . . . . . . 74

4.2.3 Specification of Relationships Between Measures . . . . . . . . 75

4.2.4 Alignment with the Quality Model . . . . . . . . . . . . 77

4.3 Ontological Representation of the Quality Model . . . . . . . . . . . . 80

4.3 .1 Conceptual Model . . . . . . . . . . . . . . . . . . . . 80

4.3.2 Extensions to Existing Ontologies . . . . . . . . . . . . 81

4.3.3 Representing the Linked Data Quality Model . . . . . . . . . 84

4.4 LD Sniffer . . . . . . . . . . . . . . . . . . . . . . . 8 85

4.5 An Example . . . . . . . . . . . . . . . . . . . . . 8 87

4.6 Future Work . . . . . . . . . . . . . . . . . . . . . . . . . . . . 89

5 Linked Data Profiling Framework $\quad 90$

5.1 Linked Data Profiling Use Cases . . . . . . . . . . . . . . . . . . . . 92

5.2 Linked Data Profiling Tasks . . . . . . . . . . . . . . . . . . . 93

5.2 .1 RDF Triple Analysis (T1) $\ldots \ldots \ldots \ldots \ldots$

5.2 .2 Vocabulary Usage Analysis (T2) . . . . . . . . . . . . . . 97

5.2 .3 Network Metrics Analysis (T3) . . . . . . . . . . . . . . . . 99

5.2 .4 Triple Pattern Analysis (T4) . . . . . . . . . . . . . . . . 101

5.2.5 Value Distribution Analysis (T5) . . . . . . . . . . . . 104

5.2 .6 Language Analysis (T6) . . . . . . . . . . . . . . . 106

5.3 Loupe Linked Data Profiling Model . . . . . . . . . . . . . . . . 107

5.4 Loupe Linked Data Profiling Tools . . . . . . . . . . . . . . 107 
5.4.1 Linked Data Profiling Toolkit . . . . . . . . . . . . . . . 108

5.4 .2 Loupe Web Portal . . . . . . . . . . . . . . . . . . . . . 111

5.4 .3 Loupe REST API . . . . . . . . . . . . . . . . . . . . . . . 112

5.4 Profiling Results Repository . . . . . . . . . . . . . . . 113

5.5 Future Work . . . . . . . . . . . . . . . . . . . 116

$6 \quad$ RDF Shape Induction $\quad 117$

6.1 RDF Shapes . . . . . . . . . . . . . . . 120

6.2 Generic Shape Induction Approach . . . . . . . . . . . . . . . . 124

6.2.1 Task Formulation . . . . . . . . . . . . . . . . . . . 127

6.2.2 Feature Engineering . . . . . . . . . . . . . . . . . 131

6.2.3 Gold Standard Preparation . . . . . . . . . . . . . . 137

6.2.4 Algorithm Selection . . . . . . . . . . . . . . . . . 139

6.2.5 Constraints and RDF Shape Generation . . . . . . . . . . . 140

6.3 Future Work . . . . . . . . . . . . . . . . . . . . . . . . . . . 142

7 Methods for Quality Assessment and Repair 143

7.1 Detection of inconsistent RDF mappings . . . . . . . . . . . . . . . . 144

7.1 .1 Use Case . . . . . . . . . . . . . . . . . . . 144

7.1.2 Approach .......................... 146

7.1 .3 Inconsistent Mappings . . . . . . . . . . . . . . . . . . 147

7.1.4 Inconsistent Mapping Prediction Method . . . . . . . . . . . . 149

7.1.5 Training the Prediction Model . . . . . . . . . . . . . . 151

7.1.6 Usage of the Prediction Model . . . . . . . . . . . . . . . . . 151

7.1 .7 Improving the Model . . . . . . . . . . . . . . . . . . . . . 154

7.2 Noise Detection in Dataset Generation . . . . . . . . . . . . . . 156

7.2.1 Use Case . . . . . . . . . . . . . . . . . . . . . 156

7.2 .2 Approach . . . . . . . . . . . . . . . . 157

7.2 .3 Types of Noise . . . . . . . . . . . . . . . . . . 158

7.2 .4 Generation of RDF Data . . . . . . . . . . . . . . . 159

7.2 .5 Noise Detection Method . . . . . . . . . . . . . . . . . 161

7.3 A Method for Repairing Links in Data . . . . . . . . . . . . . . . 162

7.3.1 Use Case . . . . . . . . . . . . . . . . . . 162

7.3.2 Approach .................... 166

7.3.3 Things as Strings . . . . . . . . . . . . . . . . 167

7.3.4 Link Reparation Technique in Data . . . . . . . . . . . . . . 169

7.3.5 Identification of Candidate Properties . . . . . . . . . . . . . . 174

7.3.6 Literal to IRI Conversion . . . . . . . . . . . . . . . . . . 175

7.4 Type Prediction using Binary Classifiers . . . . . . . . . . . . . 176

7.4 .1 Use Case . . . . . . . . . . . . . . . . . . 176

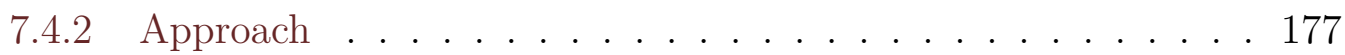


7.4.3 Feasibility analysis . . . . . . . . . . . . . . . 178

7.4.4 Binary Classifiers as Type Predictors . . . . . . . . . . . . . . 180

7.4.5 Feature Engineering . . . . . . . . . . . . . . . . 181

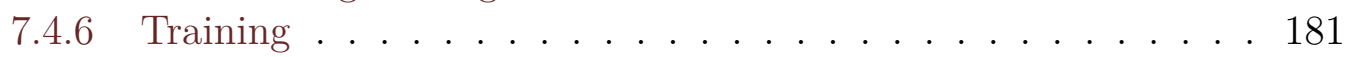

8 Evaluation $\quad 183$

8.1 Linked Data Quality Model (LDQM) . . . . . . . . . . . . . . . 184

8.1.1 Properties of the Quality Model . . . . . . . . . . . . . . . 184

8.1.2 Usefulness and Applicability of the Quality Model . . . . . . . 186

8.2 A Framework for Linked Data profiling (Loupe) . . . . . . . . . . . . 191

8.2.1 Expressivity of the Loupe Extended Dataset Description Model 191

8.2.2 Scalability of the Loupe Tools . . . . . . . . . . . . . . . 198

8.3 RDF Shape Induction Method . . . . . . . . . . . . . . . . . . 205

8.4 Methods for Quality Assessment and Repair . . . . . . . . . . . . . . 211

8.4.1 Prediction of Inconsistent Mappings . . . . . . . . . . . . . . . 212

8.4.2 Noise Detection in Information Extraction . . . . . . . . . . . 218

8.4.3 Repairing Hidden Links . . . . . . . . . . . . . . . . . . . . . 221

8.4.4 Type Prediction Method using Binary Classifiers . . . . . . . 225

9 Conclusions and Future Work 231

9.1 Review of Main Contributions . . . . . . . . . . . . . . . . 231

9.2 Future Work . . . . . . . . . . . . . . . . . . 236

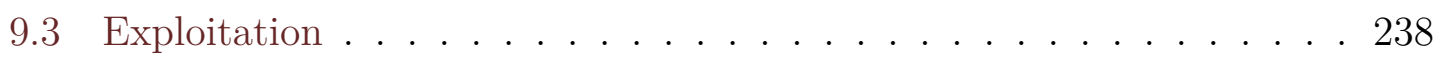

9.3.1 Linked Data Profiling Framework . . . . . . . . . . . . . . . 238

9.3.2 RDF Shape Induction Method . . . . . . . . . . . . . . . 243

$\begin{array}{ll}\text { Bibliography } & 245\end{array}$ 


\section{List of Figures}

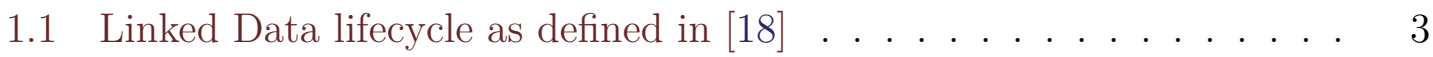

1.2 Linked Open Data (LOD) Cloud as of March 2019 . . . . . . . . . 5

1.3 Linked Data quality lifecycle . . . . . . . . . . . . . . . 7

1.4 Linked Data generation and exploitation . . . . . . . . . . . . 15

3.1 Thesis overview . . . . . . . . . . . . . . . . . . . 58

4.1 Aspects of Linked Data quality . . . . . . . . . . . . . . . . 70

4.2 Data model (RDF) levels . . . . . . . . . . . . . . . . . . . . . . . . . . . . . 71

4.3 Subset of the Linked Data quality model . . . . . . . . . . . . . . 78

4.4 The conceptual model . . . . . . . . . . . . . . . . . . 81

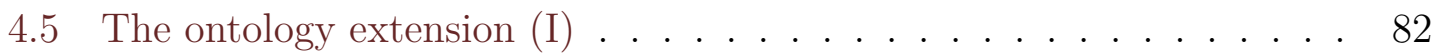

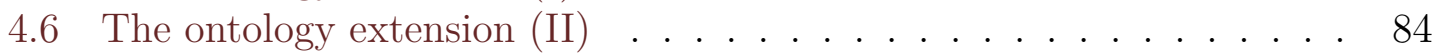

4.7 LD Sniffer web interface . . . . . . . . . . . . . . . . . . . . . . . . . . . 85

4.8 LD Sniffer docker container . . . . . . . . . . . . . . 86

5.1 Relationship between Linked Data profiling tasks, metrics, and use cases 94

5.2 An example of in-degree and out-degree metrics . . . . . . . . . . . 100

5.3 An example of 3 components in an RDF graph . . . . . . . . . . . . 100

5.4 An example of abstract triple patterns . . . . . . . . . . . . . . . 102

5.5 The Loupe conceptual model . . . . . . . . . . . . . . . . . . . . . . . 108

5.6 Architecture of Loupe Linked Data profiling tools . . . . . . . . . . . 110

5.7 Loupe web portal . . . . . . . . . . . . . . . . . . . . . . . . . . . . . . . . . 111

5.8 Loupe API documentation . . . . . . . . . . . . . . . . 113

6.1 RDF Shape induction using Linked Data profiling metrics . . . . . . 117

6.2 SHACL-Core components . . . . . . . . . . . . . . . . . . 122

6.3 A method for RDF Shape induction. . . . . . . . . . . . . . 127

6.4 Histogram of object types of dbo:Person-dbp:birthPlace . . . . . . . 136

6.5 String length distribution of foaf:Person-foaf:name . . . . . . . . 138

7.1 Detecting inconsistent mappings . . . . . . . . . . . . 147 
7.2 Data from correct and consistent mappings . . . . . . . . . . . . . 148

7.3 A screenshot from domain expert annotator web app . . . . . . . . 155

7.4 Information extraction output . . . . . . . . . . . . . 156

7.5 The noise detection workflow . . . . . . . . . . . . . . . . 157

7.6 Types of noise . . . . . . . . . . . . . . . . . . . . . 158

7.7 Noise detection workflow . . . . . . . . . . . . . . . . 161

7.8 RDF graphs with (a) literals and (b) IRIs . . . . . . . . . . . . . . 164

7.9 Examples of an entity relation and a non-entity relation . . . . . . . 165

7.10 Link repair method workflow . . . . . . . . . . . . . . . . . 166

7.11 Type prediction method . . . . . . . . . . . . . . 177

8.1 Example of dataset evaluation results . . . . . . . . . . . . . . 188

8.2 Average dereferenceability of dbo:Person/dbo:Place instances . . . . . 189

8.3 Distribution of HTTP status codes for non-dereferenceable IRIs . . . 191

8.4 Execution time (in seconds) Vs dataset size (in millions of triples) . . 200

8.5 Log of avg time per triple (in milliseconds) Vs dataset size (in millions of triples . . . . . . . . . . . . . . . . . . . . . 201

8.6 Correlation between dataset size and execution time . . . . . . . . . . 201

8.7 Execution time (in seconds) Vs total vocabulary terms . . . . . . . . 202

8.8 Execution time (in seconds) Vs total vocabulary terms . . . . . . . . 203

8.9 Correlation between the total number of vocabulary terms and execution time. . . . . . . . . . . . . . . . . . 203

8.10 Execution time (in seconds) Vs number of classes . . . . . . . . . . 205

8.11 Execution time (in seconds) Vs number of properties . . . . . . . 205

8.12 Selected projection to 2D of the 4D optimized PCA space. Blue crosses are Incorrect predictions. The ellipse shows a high concentration of Incorrect predictions that could explain the good classification of the model. . . . . . . . . . . . . . . . . . . . . . . 219

8.13 Ranking of component sizes in the original graph. Components with more than 100 nodes are labelled with the number of nodes. . . . . . 225

8.14 Identifying Holidays in a knowledge graph. Right) in a mix of Holidays and Persons, and Left) in a mix of Holidays, Persons and Events. Notice that the colors for instances of Holiday and Person differ in each graph. . . . . . . . . . . . . . . . . . . . . . . . 229 


\section{List of Tables}

1.1 A summary of publications . . . . . . . . . . . . . . . . 17

1.2 Registered software . . . . . . . . . . . . . . . . . 21

2.1 Linked Data quality assessment tools . . . . . . . . . . . . . 37

4.1 Linked Data quality model characteristics and indicators. . . . . . . . 79

5.1 Linked Data profiling use cases . . . . . . . . . . . . . . . . . . . . . . 92

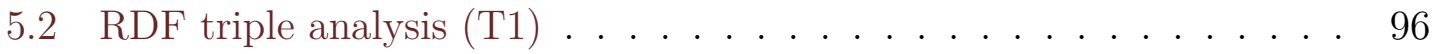

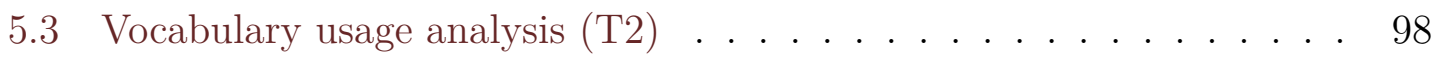

5.4 Network metrics analysis $(\mathrm{T} 3)$. . . . . . . . . . . . . . . . . . 101

5.5 Triple pattern analysis $(\mathrm{T} 4)$. . . . . . . . . . . . . . . . . . . . . . . . . . . . . . . . 102

5.6 dbo:Movie-dbo:length value distribution . . . . . . . . . . . . . . . 104

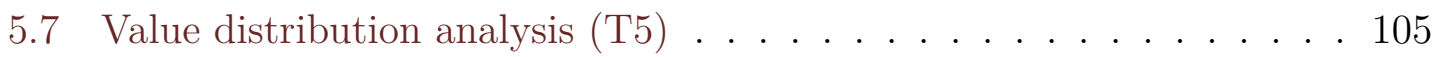

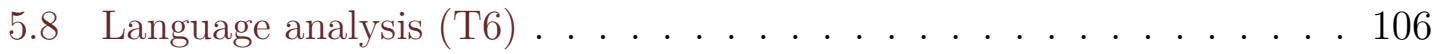

5.9 Overview of Loupe profiling results repository . . . . . . . . . . . . . 114

5.10 Most widely used vocabularies in datasets . . . . . . . . . . . . . . . 114

5.11 Most common instances of classes . . . . . . . . . . . . . . . . . . . . . . . . . . . 115

5.12 Most commonly used properties . . . . . . . . . . . . . 115

6.1 Constraints in SHACL . . . . . . . . . . . . . . . . . . . . . 124

6.2 Minimum and maximum cardinality levels . . . . . . . . . . . . 128

6.3 Object node kind examples . . . . . . . . . . . . . . . . . . . . . . . 129

6.4 Value type examples . . . . . . . . . . . . . . . . . . . 129

6.5 String-based constraint examples . . . . . . . . . . . . . . 130

6.6 Cardinality counts for dbo:Sport-dbo:union class-property. . . . . . . . 131

6.7 dbo:Sport-dbo:union P1 to P13 statistical measures from the raw cardinality distribution . . . . . . . . . . . . . . . . . . 132

6.8 dbo:Sport-dbo:union P14 to P20 statistical measures from distinct cardinality values distribution . . . . . . . . . . . . . 133 
6.9 dbo:Sport-dbo:union P20 to P30 statistical measures from cardinality percentage distribution . . . . . . . . . . . . . . . 133

6.10 Object node type information . . . . . . . . . . . . . . . . . 134

6.11 Type of dbo:Person-dbp:birthPlace objects . . . . . . . . . . . . 135

6.12 Datatypes of dbp:Person-dbp:deathDate literals . . . . . . . . . . 135

6.13 Frequency distribution of schema:Language/dbo:iso6393Code property. 137

6.14 Frequency distribution of foaf:Person-foaf:name property. . . . . . . . 137

7.1 Quality assessment and repair use cases . . . . . . . . . . . . . . 143

7.2 Data from an incorrect mapping . . . . . . . . . . . . . . . . . . 149

7.3 A snippet from English-Spanish mapping annotation . . . . . . . . . 152

7.4 Summary of the results of applying the predictive model (EN-ES literals) to the IRIs dataset . . . . . . . . . . . . . . . . 153

7.5 Detailed accuracy data and confusion matrix for the predictive model (EN-ES literals) on the IRIs dataset . . . . . . . . . . . . . . . 153

7.6 Summary of the prediction accuracy for other language-pairs. . . . . 154

7.7 Class-property profile definition . . . . . . . . . . . . . . . . . . 172

7.8 Top entity relations with strings in LOD Cache . . . . . . . . . . . 174

7.9 Top entity relations with strings in WDC . . . . . . . . . . . . 175

7.10 Top types of entities as strings in DBpedia . . . . . . . . . . . . . . 175

7.11 Literal to IRI conversion . . . . . . . . . . . . . . 176

8.1 List of errors found in non-dereferenceable IRIs . . . . . . . . . . . . 190

8.2 Domains with most non-dereferenceable IRIs . . . . . . . . . . . . . . 190

8.3 State-of-the-art dataset description models . . . . . . . . . . . . . . . 192

8.4 Summary of metrics coverage . . . . . . . . . . . . . . . . 193

8.5 T1 - RDF triple analysis . . . . . . . . . . . . . . . . 194

8.6 T2. Vocabulary usage analysis . . . . . . . . . . . . . . . 195

8.7 T3. Network metrics analysis . . . . . . . . . . . . . . 196

8.8 T4. Triple pattern analysis . . . . . . . . . . . . . . . 197

8.9 T5. Value distribution analysis . . . . . . . . . . . . . . . 197

8.10 T6. Language analysis . . . . . . . . . . . . . . . . . . . . . 198

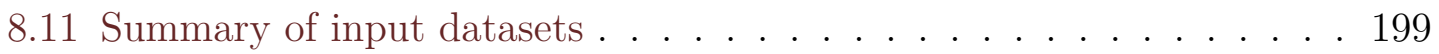

8.12 Summary of vocabulary term counts . . . . . . . . . . . . . . . 201

8.13 Summary of classes used and properties used in each dataset . . . . . 204

8.14 Cardinality class imbalances . . . . . . . . . . . . . . 207

8.15 Integrity constraints performance measure for English DBpedia . . . 208

8.16 Cardinality constraints performance measure for Spanish DBpedia . . 209

8.17 Cardinality constraints performance measures for 3cixty . . . . . . . . 209

8.18 Range constraints performance measure for the English DBpedia dataset.210

8.19 Range constraints performance measure for the Spanish DBpedia dataset.211 
8.20 Range constraints performance for the 3Cixty dataset. . . . . . . . . 211

8.21 English-Spanish literals results . . . . . . . . . . . . . . . . 215

8.22 Summary of classifiers output. . . . . . . . . . . . . . . . . 216

8.23 Summary of the results of applying the predictive model (EN-ES

literals) to the IRIs dataset . . . . . . . . . . . . . . . 216

8.24 Detailed accuracy data and confusion matrix for the predictive model

(EN-ES literals) on the IRIs dataset . . . . . . . . . . . . . . . 217

8.25 Summary of the prediction accuracy for other language-pairs . . . . . 217

8.26 Principal Components Analysis (PCA): Effect of reducing the number of PCA attributes on the accuracy of the predictive model . . . . . 218

8.27 Experimental results . . . . . . . . . . . . . . . . . . . 220

8.28 Candidate identification performance . . . . . . . . . . . . . . . . . . . . . . . . 223

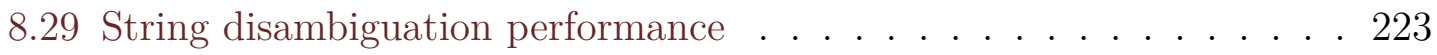

8.30 Improvements in connectivity . . . . . . . . . . . . . . . . 224

8.31 Possible predictions of type prediction model for class X . . . . . . . 226

8.32 Datasets used in the experiments . . . . . . . . . . . . . . . 227

8.33 Results of experiment I . . . . . . . . . . . . . . . . . . . . . . . . . . . . . . . . . . . . . . . . . . . . . 228

8.34 Results of experiment II . . . . . . . . . . . . . . . . . . . . . . . 229

8.35 Results of experiment III . . . . . . . . . . . . . . . . . 230 


\section{Chapter 1}

\section{Introduction}

The World Wide Web (WWW) has made a significant impact in the world because it changed the way we share information by allowing people to publish documents into a global information space that is accessible by millions of people. Furthermore, because these Web documents has the capability to embed hypertext links which enabled readers of these documents to traverse to other related documents to find more contextual information. Since its inception, the Web contains a large volume of valuable information generated by organisations such as companies, libraries and other educational institutions, as well as individual web users. For instance, Wikipedia ${ }^{1}$, a collaborative crowd-sourced encyclopedia, contains more than 45 million pages in 301 languages and is growing each day with a lot of new information.

Similarly, social-media and other platforms of user-generated content are on the rise. Nevertheless, not all of the information shared on the Web is of high quality. For example, the rise of Fake News [12] provides good evidence that the growth of quantity does not necessarily entail quality, and it could even be the contrary. Thus, to effectively use the data available on the Web and the information such data provides, it is essential to have quality assessment techniques so that high-quality data can be identified and used while low-quality data can be either eliminated or improved to satisfy better necessary quality standards.

In the current era of digital transformation, data, from which useful information is derived, plays a vital role both in academia as well as in industry. Though during its early years the Web was mainly used to share documents intended for human readers, this gradually changed with the advancement of information technology, especially in areas such as artificial intelligence. Currently, data represented in a way that can be easily processed and consumed by machines has become an important part of the Web leading to the Web of Data [30, 33].

In a recent article, The Economist magazine stated that the world's most valuable

\footnotetext{
${ }^{1}$ https://www.wikipedia.org/
} 
Chapter 1. Introduction

resource is no longer oil, but data ${ }^{2}$ (the phrase, "data is the new oil", was credited initially to Mathematician Clive Humby back in 2006). The increasing capabilities to process, transfer, and store huge volumes of data with powerful computing infrastructure and fast network connections have made big data a key source of new economic value and innovation [125]. One of the crucial factors of data to become useful is their quality. This is because the quality of the results of most data-driven models and applications, including machine learning and deep learning will depend on the quality of the data being used. Most applications of data follow the "garbage in, garbage out" pattern, so if the input data is concerned by quality issues, then that will lead to undesirable results.

The Resource Description Framework (RDF) [52] is a graph-based data model. It is published by the World Wide Web Consortium (W3C) as a W3C recommendation, and it is widely used in Semantic Web and Linked Data applications. RDF graphs contain triples which are statements about relationships between two resources. These statements represent facts about certain entities in a semantically rich manner.

The Linked Data principles ${ }^{3}$, which were introduced by Tim Berners-Lee, promote publishing structured data and interlinking them in a machine-readable manner using Web standards. Linked Data, along with the other Semantic Web technologies, allows data to be interlinked and reused across organisational boundaries instead of being data silos used by a single organisation. Linked data has several advantages over other data paradigms [161], namely: (a) global identifiers for data that can be accessed using the Web infrastructure and typed links between data from different applications; (b) the graph-based RDF data model that allows consuming and merging data from different sources without having to do complex structural transformations; and (c) explicit semantics of data expressed in RDF Schema or OWL ontologies which can be aligned or mapped to data models of other applications using techniques such as ontology matching. However, along with these benefits, Linked Data introduces a new set of challenges for data quality concerning different aspects [208, 235, 237].

Several Linked Data Life Cycles have been proposed over the years in the Semantic Web literature. The one proposed by Villazon-Terrazas et al. [226] is an iterative, incremental life cycle consisting of five phases: (a) specify phase (in which activities such as URI design, analysis of the data sources, and definition of provenance information are performed) (b) model phase (in which suitable ontologies are identified and reused to create a model to represent the data) (c) generate phase (in which data sources are transformed into RDF, linked to other datasets) (d) publish phase (in which data and metadata are published in compliance with the Linked Data standards), and finally (e) exploit phase (in which data is used by downstream

\footnotetext{
${ }^{2}$ https : //Www. economist. com/leaders/2017/05/06/the-worlds - most - valuableresource-is - no- longer-oil - but - data

${ }^{3}$ https://wWw.w3.org/DesignIssues/LinkedData.html
} 
applications). Similarly, Atemezing et al. [16] proposed a life cycle consisting of five phases (a) dataset and ontology selection, (b) data conversion, (c) data protection, (d) data interlinking, and (e) data publication. The one proposed by Hyland and Wood [112] follows a similar approach with seven steps including (a) identify, (b) model, (c) name, (d) describe, (e) convert, (f) publish, and (g) maintain. It is important to note that these lifecycles do not mention quality assurance or repair as one of their phases. As empirical studies [46, 58, 104, 110, 111, 121, 158] show, this has lead to datasets that are mostly concerned quantity of data generated than the quality of data. The Linked Data Lifecycle proposed by Auer et al. [18] is the first one to include quality assessment and repair as two separate phases in the lifecycle. It consists of 8 phases including (a) extraction, (b) storage/querying, (c) manual revision/authoring, (d) interlinking/fusion, (e) classification/enrichment, (f) quality assessment, (g) evolution/repair, (h) search/browsing/exploration as illustrated in Figure 1.1.

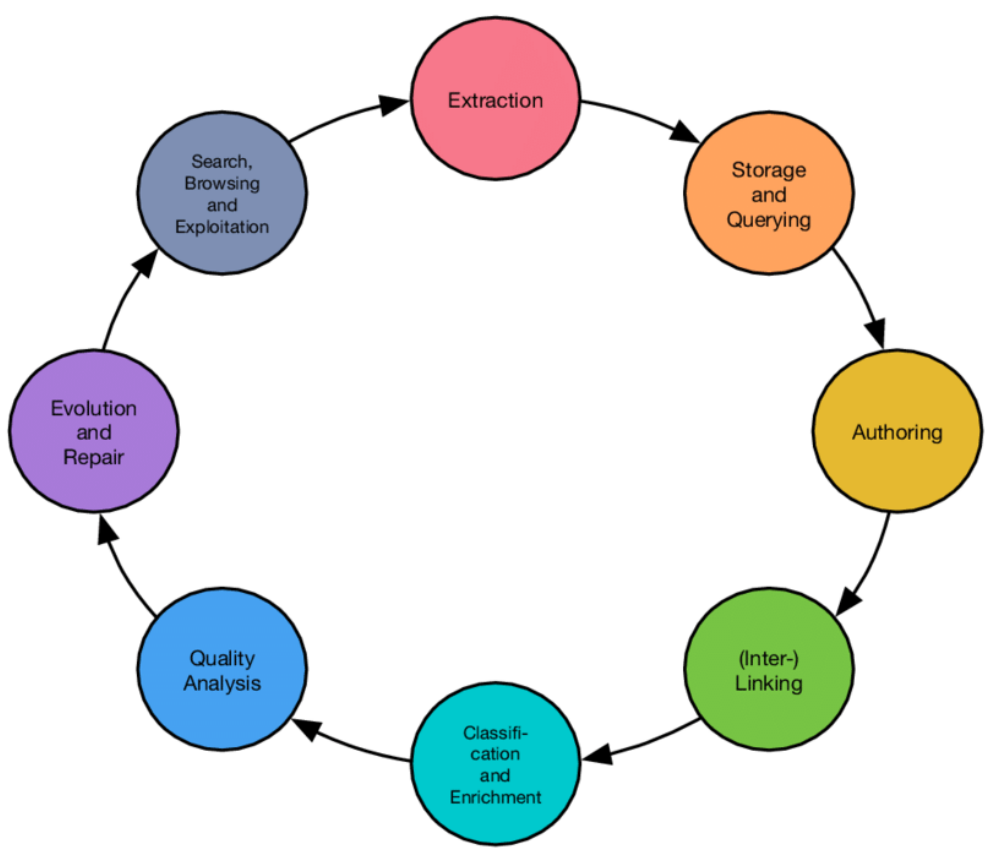

Figure 1.1: Linked Data lifecycle as defined in [18]

As discussed before, the process of Linked Data generation generally includes data transformation from original sources, mapping data to several vocabularies or ontologies, and fusion and interlinking of data from different data sources. Each of these steps can open the door to possible data quality issues in many different aspects [58, 111, 235], such as dereferenciability of resource identifiers (IRI) [58, 59, 
166] or vocabularies used to describe data and their semantic accuracy [148, 188], conciseness [34, 117], consistency [29, 122, 174], completeness [116]. Furthermore, the process of Linked Data generation generally includes data transformation steps, mapping data to several vocabularies or ontologies and fusing data from different data sources, which opens the door to possible data quality issues [62, 63, 128, 189, 200].

The outcome of such Linked Data generation processes is generally an RDF dataset that is accessible through the Web using their IRIs. DBpedia [17], Wikidata [227], Freebase [38], and Yago [197] are examples of a few encyclopedic Linked Data knowledge bases that are widely used. Among themselves, they contain roughly around 5 billion triples. Furthermore, the Linked Data Cloud project ${ }^{4}$ (see Figure 1.2) lists 1,239 datasets (as of March 2019) of different sizes. Similarly, LOD Laundromat ${ }^{5}$ [24], which provides a repository of cleaned datasets lists $650 \mathrm{~K}$ datasets containing 28 billion unique triples [76]. Web Data Commons [175], which is a dataset created by extracting structured data from the Common Crawl web corpus containing 2.45 billion pages, consist of 44.2 billion triples in different formats such as Microdata ${ }^{6}$, JSON-LD ${ }^{7}$, or $\mathrm{RDFa}^{8}$. These statistics provide an indication of the sheer amount of Linked Data generated and the need for scalable approaches for assuring the quality of such data.

Quality is well recognised as a crucial need across domains (e.g., civil engineering [181], software engineering [130]), and in order to provide high-quality products and services, the specification and evaluation of quality are of high importance [25]. Similarly, data is a pivotal asset in many domains such as medicine, education or government, and the importance of data quality has led to different data quality legislations such as the US Data Quality Act [182] or the BCBS 239 Data Quality Mandate [51]. Furthermore, the success of business processes where decision making is based on data analytics depends mainly on the quality of data [114]. While the literature describes different definitions of data quality, the general notion is that data quality is tightly connected to the intended use and usefulness of data. The ISO defines data quality as a "key component of the quality and usefulness of information derived from that data" [114], while Juran and Godfrey define it as a "fit for intended use in operations, decision-making, and planning" [129]. Nevertheless, usefulness is not the criterion for evaluating data quality. There are some intrinsic characteristics, such as consistency or conciseness [235] that have to be considered. Following this discussion, with an increasing amount of data available on the Web as Linked Data, the quality of Linked Data datasets is of great concern.

\footnotetext{
${ }^{4}$ https: //lod-cloud.net/

${ }^{5}$ http : //lodlaundromat.org/

${ }^{6}$ https: //WwW.w3.org/TR/microdata/

${ }^{7}$ https : //WwW.w3.org/TR/j son - ld/

${ }^{8}$ https : //WwW.w3 . org/TR/html - rdfa/
} 


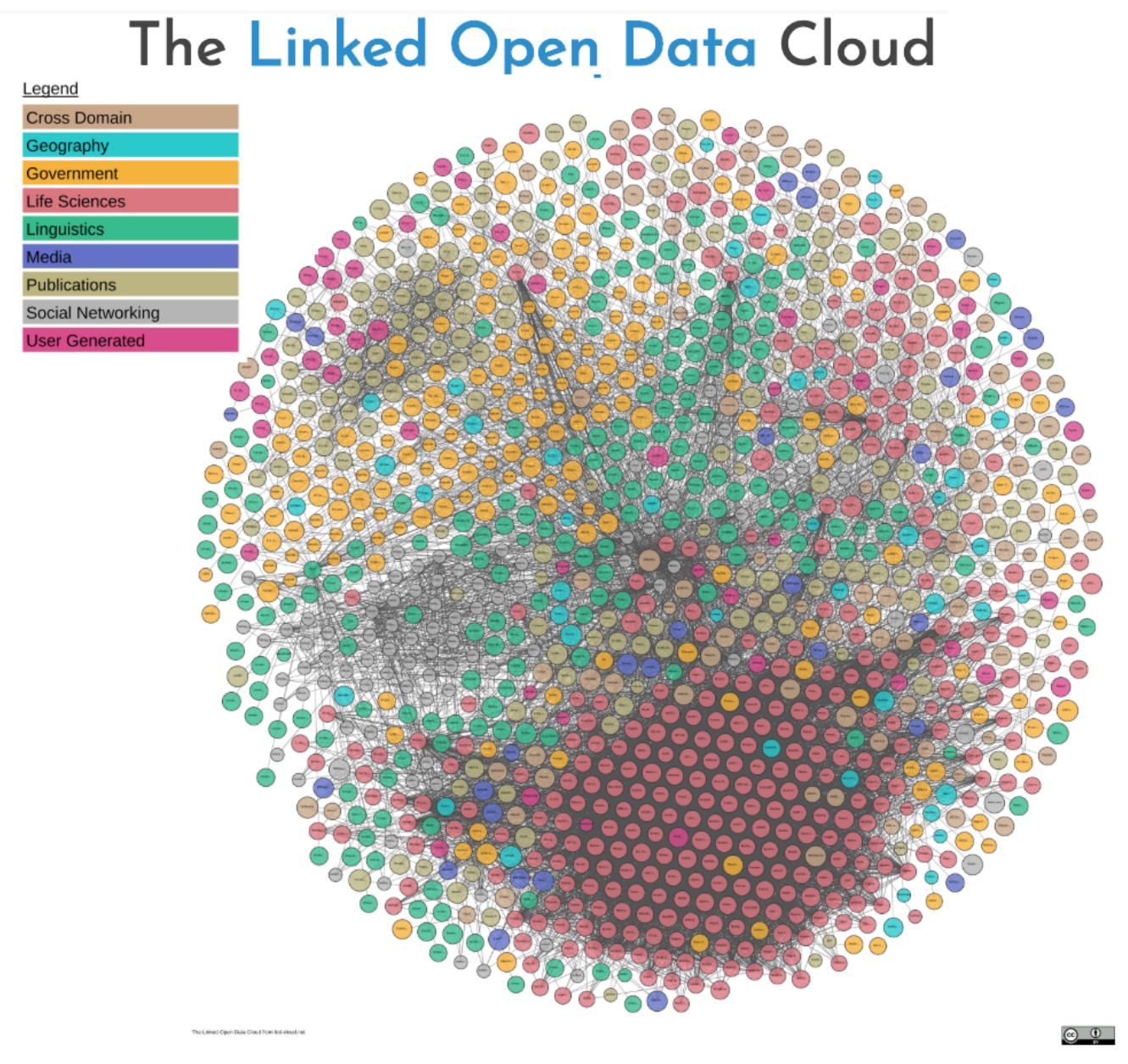

Figure 1.2: Linked Open Data (LOD) Cloud as of March 2019

Furthermore, it is not enough to perform the quality assessment only on the generated outputs of the Linked Data. As the process of Linked Data generation can involve many phases such as ontology definition, data conversion and population, interlinking, publication on Web, it might require quality assessment to be preformed at each of these phases. For instances, the quality issues related to mappings might be identifying efficiently by evaluating the data conversion phase.

During the last decade, there has been a massive growth of Linked Data with the big enthusiasm in creating a Web of Data by publishing data in a manner that can be easily understood by software programs. However, it is evident that a systematic process for ensuring the quality of data has not been followed in Linked Data generation and publication resulting increase mainly in quantity but not in quality $[46,58,104,110,111,121,158]$. As a consequence, validating the quality of Linked Data and their potential repair has become an important topic in the Linked 
Chapter 1. Introduction

Data research community.

To address this need, several Linked Data quality dimensions and metrics have been proposed over the years [221, 235] and some standards languages such as Shapes Constraint Language (SHACL) and Shape Expressions (ShEx)[93] have been established. Furthermore, several promising tools such as Sieve [157], RDF Unit [142], LOD Laundromat [24], Luzzu [57], Triplecheckmate[141] and others have been developed. Nevertheless, as we discuss in the following sections, there are still gaps in state of the art in Linked Data quality assessment. As we will discuss in detail in the following sections, we need standard quality metrics that are comparable across different datasets. Furthermore, some of the quality assessment techniques require large manual effort and do not scale to large datasets. While there is a large number of research studies that are focused on quality assessment, the research of repair techniques is still rather limited. More discussion on open research problems are presented in detail in Section 3.2.

The main focus of this thesis is to advance the state of the art of the Linked Data quality assessment and repair research by proposing new methods and techniques.

\subsection{Linked Data Quality Lifecycle}

In the previous section, we discussed several Linked Data lifecycle definitions[16, 18, $112,226]$ consisting of several phases such as data extraction, transformation, storage, publication, interlinking, and enrichment. Only one of them [18] explicitly included quality assessment and repair phases. In contrast to [18], rather than having quality assessment and repair at the end of the Linked Data generation lifecycle, we propose that quality assessment and repair can be performed in all different phases of the lifecycle. Furthermore, we propose that the quality analysis can be considered to have a lifecycle on its own, as illustrated in Figure 1.3. This proposed Linked Data quality lifecycle applies equally to ontologies, instance data, as well as metadata as represented in the diagram.

The first phase of the quality lifecycle is to define a quality model with a set of metrics that can be used to access the quality of Linked Data for a given use case. Thus, a need for a quality model arises from the early stages of the quality lifecycle [229]. A quality model is defined through a set of specific quality characteristics, quality measures, and through the relationships between these characteristics and measures. To this extent, it represents a specification of quality-related information [114]. Quality models are essential for providing consistent terminology and guidance for quality assessment and are the basis for the evaluation of Linked Data for a given use case. This is especially significant for the integration of evaluation results and benchmarking, which is one important aspect of evaluation [91, 136], and without a quality model, it is sometimes difficult to integrate evaluation results, to perform 
benchmarking, or to select datasets according to their quality.

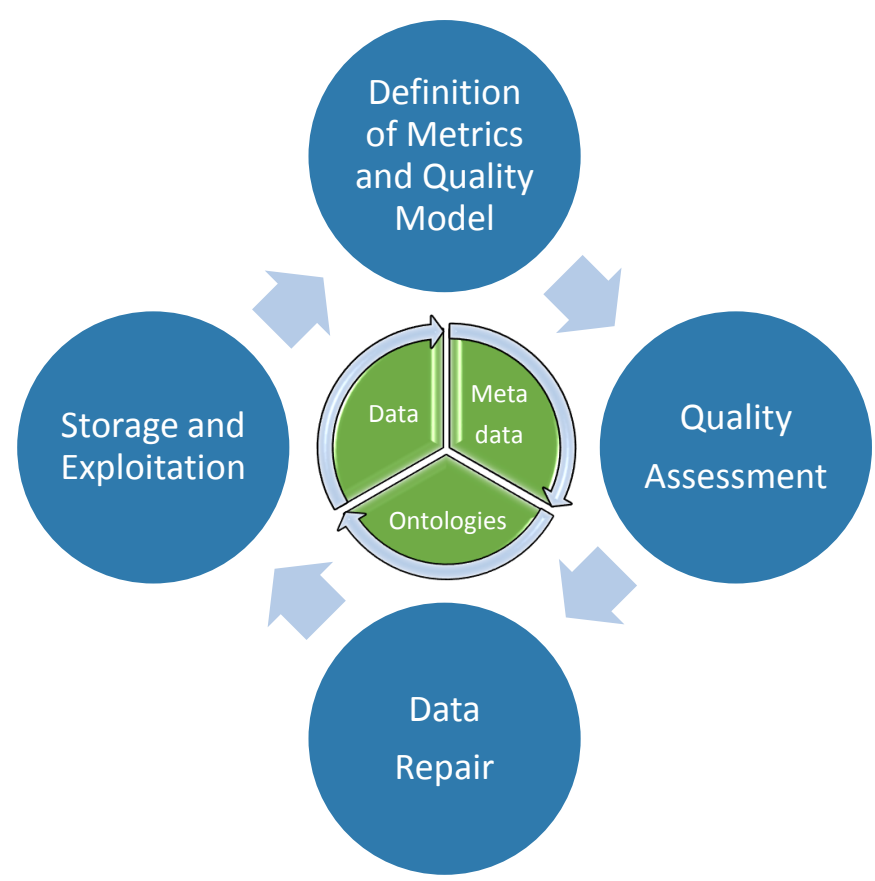

Figure 1.3: Linked Data quality lifecycle

The next step of the Linked Data Quality Lifecyle is to perform the quality assessment. Quality can be assessed in different ways, for example, manually evaluating the data with some criteria such as checklists or automatically with the help of automated tools [240]. Manual evaluations can yield good results with respect to the accuracy of the evaluation but such evaluations require large amounts of human labour as these evaluations can be time-consuming [176]. Thus, such methods could be useful for small datasets but not feasible for large datasets. Automated techniques with tool support scale much better with minimal human efforts for large datasets, but the current automated techniques are mostly used for less complex tasks such as identifying syntax errors [24]. It is easier to build an automated tool for detecting syntax errors in a large dataset compared to building an automated tool for detecting if a given fact in the data is accurate and up-to-date. Thus, it is challenging to develop automated tools for tasks that require complex human knowledge.

Furthermore, crowd-sourcing techniques can be used to scale the tasks with the help of a large pool of human users so that manual techniques can be used with larger datasets [5, 141, 224, 236]. Nevertheless, this requires the human users to have certain general knowledge or in some cases expert knowledge depending on the quality issues expected to be assessed [233]. Thus, quality assessment of large datasets still 
Chapter 1. Introduction

remains a challenging task.

Once quality assessment is performed on a dataset and the quality problems are identified, the next stage of the Linked Data quality lifecycle is to clean and repair the data [102]. This step supports the evolution of data and allows the data to become more useful. There are several strategies for repairing data. On the one hand, one approach is to detect the erroneous data and eliminate them. For example, given an RDF dataset, any triples that contain syntax errors can be identified and removed from the dataset so that the resulting dataset will be free of syntax errors [24]. However, in this case, there will be a loss of information because the repair process can remove a significant amount triples that contained syntax errors.

On the other hand, another approach is to detect the errors and attempt to repair them by correcting the syntax errors without removing those triples. In this case, the information contained in the erroneous triples will not be lost as those triples will remain in the dataset once they are corrected. Choosing the right strategy for repairing Linked Data and resolving the quality issues of Linked Data also remains a challenging task.

Once the quality assessment and repair tasks are performed on data, the clean data can be stored and exploited for their intended purposes. Not only the cleaned data but also the quality assessment results as well as the intermediate data produced by the quality assessment procedures can be useful for future quality assessments [73]. Thus, all such useful information could be stored and exploited effectively.

By analysing the current literature (please refer to Chapter 2), we have identified several challenges related to the Linked Data Quality Lifecycle. There is a lack of a quality model for Linked Data that specifies a set of quality characteristics and metrics related to those quality characteristics in the domain of Linked Data with a unique terminology as a reference for data quality specification and evaluation. Furthermore, there is existing work on defining data quality in general by standardisation bodies such as the International Organisation for Standardisation (ISO) or the World Wide Web Consortium (W3C). Nevertheless, existing quality characteristics and metrics for Linked Data found in the literature [235] are not aligned with the models proposed by such standardisation initiatives.

In order to develop automated quality assessment techniques that scale to large amounts of data, data profiling is widely used in other paradigms such as relational data [3]. Data profiling information can provide valuable inputs to quality assessment techniques, but the current Linked Data profiling techniques $[2,4,28,75,133,149,214]$ do not extract information with the level of detail required for quality assessment techniques. For example, currently Linked Data profiling is aimed at providing metadata for tasks such as dataset summarization [214], identification of the domain of a dataset [4], visualization [149], 
revealing the redundancy for data compression [75]. Furthermore, by analysing the state-of-the-art dataset description models such as VoID [132] or DCAT [152], it is evident that the current RDF dataset description models fail to represent fine-grained information about the vocabularies used and frequent patterns in datasets.

Quality assessment and repair techniques often require a set of validation constraints [93]. One type of such validation constraints are identified as RDF Shapes [93, 210]; they can be used to derive a variety of techniques for data quality assessment and repair. Nevertheless, it is a tedious task to generate these validation constraints manually for larger datasets. One possible approach to address this challenge is to automatically induce validation constraints using data profiling and analysing patterns in data. Nevertheless, there is a lack of methodologies for inducing integrity constraints from data and generating validation inputs, such as RDF Shapes.

As we will discuss in Chapter 2, comparatively there is less research done on repairing Linked Data compared to assessing Linked Data. Even the promising Linked Data cleaning tools such as LOD laundromat [24] focus mainly on low-level issues, such as eliminating syntax errors. However, repairing Linked Data is as important as assessing them. Currently, there is a lack of repair techniques for data quality issues in Linked Data.

With this background, the general objective of this thesis is to propose novel and scalable methods for automatic quality assessment and repair of Linked Data. The motivation for proposing automatic methods and techniques is to significantly reduce the manual effort required in Linked Data quality assessment and repair tasks, thus proposing techniques suitable for large-scale Linked Data sources containing millions of triples.

To this end, four main objectives of the thesis are defined as:

- O1: The first objective of the thesis is to provide means for representing quality assessment results along with all associated metadata in a comparable manner using well-established standards. More concretely, the goal is to propose a quality model for Linked Data quality specification, adhering to the ISO standard for data quality [114] and the W3C Data Quality Vocabulary [10] so that the quality assessment results about distinct datasets can be published in a standard and interoperable manner. By using a quality model, Linked Data quality assessment results produced by many different parties and tools will be comparable. Furthermore, such models will make it possible to measure and describe the improvements made by repair techniques in a standard manner. 
- O2: The second objective is to identify information that could be automatically generated on large-scale datasets and could be used for quality assessment and repair tasks. More concretely, the goal is to improve RDF data profiling techniques focusing on the Linked Data quality assessment and repair use cases. For that, the current RDF data profiling techniques can be extended to include fine-grained profiling information that is needed for quality assessment. Furthermore, the goal is to propose an RDF dataset description model for representing data profiling information, dataset statistics, and other information about patterns in data with a sufficient level of detail. Such data profiling techniques and dataset description models will enable the use of data profiling information in Linked Data quality assessment and repair.

- O3: The third objective is to propose methods for automatically deriving Linked Data validation constraints for large datasets. More concretely, the goal is to develop an automatic RDF Shape Induction algorithm to generate constraints using data-driven approach without requiring manual effort and to combine the aforementioned constraints into RDF Shapes. Data validation constraints and RDF Shapes are induced using machine learning techniques over data profiling metrics. These RDF Shapes will provide the necessary inputs for automatic data quality assessment and repair techniques.

- O4: The fourth objective is to develop methods for automatic quality assessment and repair using data validation constraints represented as RDF Shapes. Such methods and techniques will reduce the manual effort required for quality assessment and repair and will enable efficient quality assessment and repair of large-scale Linked Data datasets such as DBpedia.

\subsection{Linked Data Quality Model}

As discussed in the previous section, the quality model is a key element of performing any quality assessment in a way that the assessment results are useful and interoperable. The ISO standardisation organisation recognised the need for a quality model for data and produced the ISO 25012 quality model [114]. However, the ISO data quality model can be regarded as very general by design, so that it can be widely used with different data models and representations. As a consequence, it does not include the particularities that are specific to Linked Data. For instance, it covers high-level quality dimensions such as accessibility or consistency but does not cover Linked Data specific aspects such as URI dereferenceability or disjoint classes. Thus, one of the objectives of this thesis is to propose a quality model taking into account 
all the aspects relevant to Linked Data in compliance with the ISO 25012 quality model.

The $\mathrm{W} 3 \mathrm{C}$ has also recognised the need for having a unified ontology for describing data quality and produced the W3C Data Quality Vocabulary (DQV) ${ }^{9}$ within the W3C Data on the Web Best Practices Working Group. Nevertheless, similar to the ISO model, DQV also aims to be a lightweight upper-level ontology suitable for any type of data on the Web (e.g., CSV, XML, HTML, RDF, etc.); thus it is designed to be generic. As a consequence, it does not provide details for any specific type of data. Furthermore, it only provides a base meta-framework for describing quality metrics and measures but does not define concrete quality metrics, which are expected to appear in quality models. Thus, the Linked Data quality model proposed in this thesis uses DQV as the base ontology for representing the quality model elements as Linked Data and extends it with the concrete quality metrics relevant to Linked Data.

Even though a large number of existing quality metrics are found in literature as discussed in the Linked Data quality assessment survey by Zaveri et al. [238], due to the lack of a quality model, those metrics can not be used in quality assessment processes in an interoperable way because the meaning of each of those metrics is not presented in a uniform manner. For instance, due to the lack of global identifiers for each metric, it will be difficult for a quality metadata consumer to know exactly which metrics were used in a given evaluation. Furthermore, the evaluation results will not be comparable as there will ambiguity between the definitions of similar metrics by different authors. Thus, the first of the objectives of this thesis is to propose a comprehensive quality model for Linked Data that provides global identifiers following the Linked Data principles and uniform definitions for the existing quality metrics using a Linked Data Quality Model ontology.

\subsection{Data Profiling}

In domains such as relational data, data profiling is widely used as a preliminary step for quality assessment and cleansing. Data profiling for relational data is defined as "the activity of creating small but informative summaries of a database" [126]. According to such definition, those summaries could include both simple statistics such as the number of records in a given table or in the whole database, as well as information derived from complex statistics, such as functional dependencies in data or the type of distribution of values in a given column of a table. In more general terms, data profiling is defined as "the set of activities and processes to determine the metadata about a given dataset" [3]. In this thesis, we focus on Linked Data profiling

\footnotetext{
${ }^{9}$ http : //www.w3 . org/TR/vocab-dqv/
} 
and the models that can represent the information generated by data profiling.

There are a large number of popular commercial tools for profiling relational data, for example, IBM's InfoSphere Information Analyzer ${ }^{10}$ or Microsoft's SQL Server Integration Services (SSIS) ${ }^{11}$. The profiling tasks in relational data, which are based on the tabular structure of relational data, are generally classified into three main categories [3]: single-column analysis, multiple column analysis, and dependency analysis. Single column analysis consists of analysing the values of an individual column for tasks such as checking the number of unique values, the number of null values, value distributions, value ranges, and common patterns. Multiple column analysis consists of tasks such as discovering correlations and association rules among different columns or clustering of similar columns. Dependency analysis consists of tasks such as identification of columns with key values or identification of foreign keys.

Even though there are several RDF data profiling tools and vocabularies [2, 4, $28,75,133,149,214]$ for representing profiling information, we do not observe data profiling being widely used in a similar way as in the relational data domain. More specifically, we argue that data profiling is under-utilised for quality assessment and repair of Linked Data. In her PhD thesis, Spahiu concludes that the current profiling tools are limited in reporting some basic statistics, after thoroughly analysing the state-of-the-art tools in Linked Data profiling [213]. Our hypothesis is that the current data profiling techniques lack the expressivity to represent the fine-grained details necessary for data quality assessment and repair use cases, and this is hindering the use for data quality. Thus, the second objective of the thesis is to improve RDF data profiling techniques focusing on the Linked Data quality assessment and repair use cases by extending the current RDF data profiling techniques to include the fine-grained profiling information that is needed for quality assessment.

\subsection{RDF Shape Induction}

RDF has proven to be a good model for data integration due to its flexibility (i.e., triples from different graphs can be easily fused together irrespective of their vocabularies used to describe them and similarly, the entities in different datasets can be easily linked to each other). There are a number of applications using RDF either for data storage or as an interoperability layer [193]. However, this flexibility also brings inherited challenges to the quality assessment and validation of data represented using the RDF model. The lack of an explicit schema in RDF data is the main reason behind this [180]. As we discuss later, ontology languages such as

\footnotetext{
${ }^{10}$ https://WWw. ibm. com/us - en/marketplace/infosphere-information-analyzer

${ }^{11}$ https : //docs . microsoft. com/es - es/sql/integration-services/sql-serverintegration-services
} 
RDF Schema (RDFS) [43] and Web Ontology Language (OWL) [109] are designed to be computational logic-based languages that allow to perform reasoning and infer new knowledge rather than schema-languages that are designed for validation.

Nevertheless, Semantic Web-related approaches, such as the RDF model are more suited for knowledge representation and reasoning. The most prominent expressive knowledge representation and reasoning methods are based on Description Logics [20]. In particular, OWL is based on Description Logics and it is a recommended standard by the World Wide Web Consortium (W3C) for modelling ontologies. Description Logics (DLs), in turn, bear a first-order predicate logic semantics. DLs are monotonic and adhere to the Open World Assumption (OWA). This means that any missing information will be interpreted as unknown information rather than false. In other words, "absence of information is not information about absence" [184]. Because of this any negations drawn from a knowledge base must be based on information explicitly present in the knowledge base rather than based on missing information.

On the other hand, under Closed World Assumption (CWA) all non-provable expressions are assumed to be false. OWA makes it challenging to perform certain validation tasks [210]. For example, a minimum cardinality constraint is not generally violated under OWA because there is always a possibility that a triple exists somewhere which will fulfil the cardinality requirement (unless such triple explicitly makes the model inconsistent).

The main reason for ontological languages to be not suitable as the descriptions of validation constraints is the fact that they are designed for inferring new knowledge using known axioms (considering all of them to be true) rather than for validating data based on the axioms. Even though reasoners can find inconsistencies in data, a reasoner and a validator have different functions, i.e., a reasoner is used for inferring new knowledge while a validator is used finding violations against a set of constraints. The underpinning principles used in Web Ontology Language (OWL) [211], such as the use of Open World Assumption and Non-Unique Name Assumption, can lead to unexpected results in a validator [210].

For example, assume that there is a constraint that a country can have only one president. If a reasoner find two distinct triples, one stating that Barak Obama is the president of United States, and the other stating that Donald Trump is the president (which is quite possible if one triple is not up-to-date and temporal validity is not correctly modelled in data), it will not find any inconsistency with only this information. That is because under the Non-Unique Name Assumption, it is possible for a single entity to have multiple names. Thus, rather than finding an inconsistency, it will incorrectly infer that Barak Obama and Donald Trump are the same person.

Most traditional data modelling paradigms such as relational databases follow a process commonly known as schema on write [64]. In such cases, a comprehensive schema with the conditions and constraints that should be held is defined before 
loading any data and a validation process is performed before every data write to ensure that the write operation does not change the integrity of the stored data. In contrast, most RDF triple stores and applications follow a more flexible model that is known as schema on read $[64,151]$. In this approach, generally only syntactic level validations are performed during data writes, and the schema of the data evolves depending on the type of data being inserted. Even though schema-on-read makes it possible to insert data with diverse schemas into RDF stores, this dynamic nature of the schema makes it harder to have a concrete set of validation constraints for a given dataset. Thus, for RDF Data, it is valuable to have approaches that can automatically discover the schema of the data at any given time.

Validation against a predefined schema to ensure that a given dataset is consistent is one of the key steps in most conventional data storage and publishing paradigms, for instance, validation against DDL constraints in SQL databases or XML Schema or RelaxNG in XML documents [193]. For RDF data, typically OWL or RDF Schema based ontologies are used for doing this kind of validations. When an ontology is available with TBox axioms that define the conceptualization of the domain, a reasoner can be used to verify whether the dataset is consistent with the domain by verifying the axioms defined in the ontology.

However, most of the ontologies do not have rich axioms that could help to detect inconsistencies in data. Most of the large knowledge bases, such as DBpedia, lack a definition of integrity constraints and it is a tedious task to create these constraint definitions from scratch manually. Furthermore, most of the schema information about RDF data is only available in the form of OWL ontologies that are most suited for entailment rather than validation. However, most practical use cases that utilise RDF data need to validate the data according to a set of integrity constraints. The use of ontologies for validating RDF data leads to several problems: (a) the mismatch between intended purposes, inference (for which the ontologies are designed for) and validation, and (b) the lack of information useful for validation in most of the ontologies. Thus, it is necessary to represent the constraints for validating data in a more suitable language.

Our third objective is to utilise the profiling information to address the challenge of inducting RDF Shapes and then to represent the induced shapes in a language suitable for representing constraints for data validation. To this end, we propose a data-driven approach by applying machine learning techniques on profiled RDF data to automatically generate integrity constraints. Then all constraints related to a given class are combined to generate an RDF Shape that is a collection of constraints. The proposed approach is defined in a generic way that applies to any type of constraints. Aligned with the main objective of this thesis, the goal of generating RDF Shapes from data is to use them for quality assessment and repair. 


\subsection{Methods for Quality Assessment and Repair}

As discussed in the Linked Data Quality Lifecycle, quality assessment and repair are two of the most important aspects of the data quality life cycle. On the one hand, data quality is multi-dimensional and a large variety of quality dimensions [190], characteristics, and measures can be defined for analysing Linked Data quality. On the other hand, data quality can be defined as "fitness for intended use in operations, decision-making, and planning" [129] putting the focus on the use case in which the data will be utilised. For instance, the data to be used for making decisions in a medical application might need a precision much higher than the data to be used in a social media application for entertainment. In this thesis, we apply quality assessment techniques to several use cases covering the different phases of the lifecycle of Linked Data generation and exploitation.

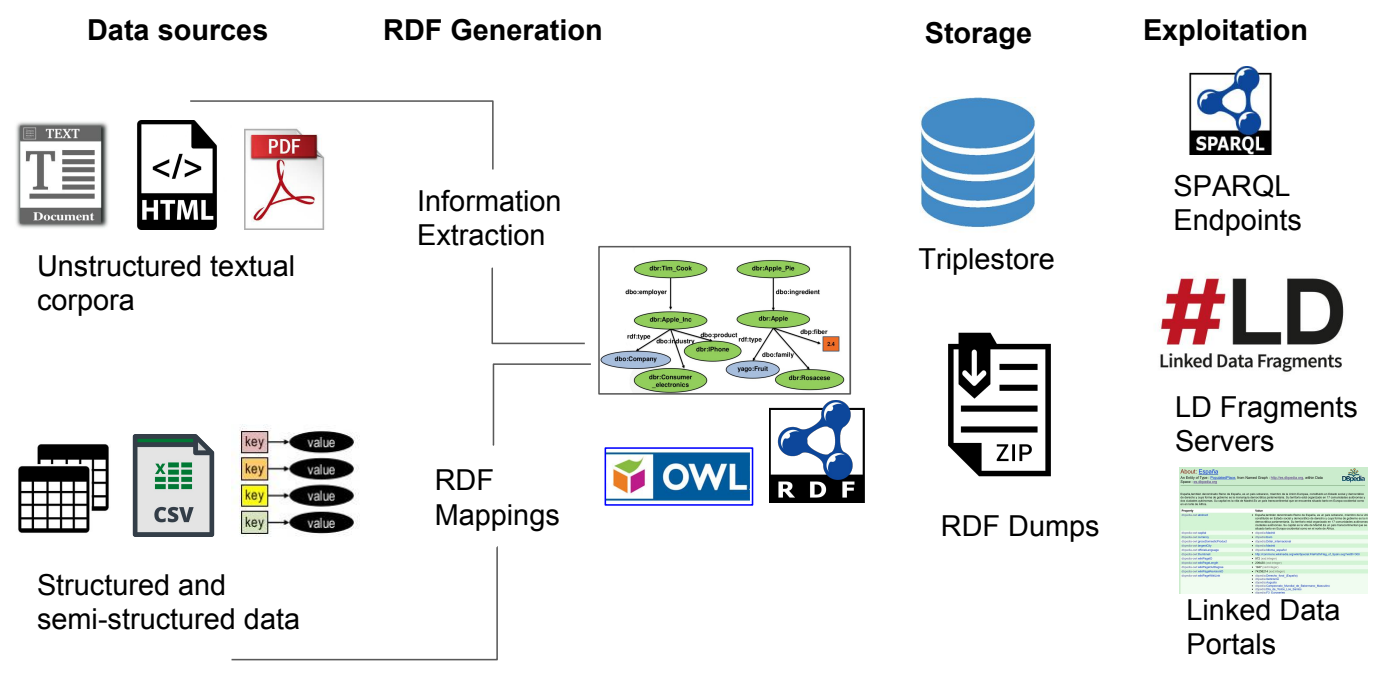

Figure 1.4: Linked Data generation and exploitation

Figure 1.4 illustrates an overview of the common steps involved in Linked Data generation and exploitation. Generally, Linked Data represented in RDF are generated using some non-RDF data sources. These data sources can be either in unstructured textual form, for example, a corpus with textual documents such as text files, PDF files, or web pages or in a structured or semi-structured form, for example, a relational database, a set of CSV files or key-value pairs. With unstructured text, information extraction techniques are used to extract facts in the form of triples (subject, predicate, 
Chapter 1. Introduction

object) which can then be represented using RDF by converting them to RDF terms. Examples of such information extraction tools include Stanford OpenIE [14], Fred [90], TextRunner [21], NELL [48] or commercial tools such as IBM's Statistical Information and Relation Extraction (SIRE) ${ }^{12}$. With structured data and semi-structured data, RDF mappings are used for mapping the data to RDF terms. One popular example is the DBpedia Extraction Framework [17] which maps the key value pairs in the Infoboxes of Wikipedia articles into RDF using a set of mappings defined for each of the Infobox templates. More recent RDF knowledge bases such as Wikidata [227] are created by users directly editing the knowledge graph using a user-friendly interface. It is possible that quality issues are introduced in the RDF generation phase and such issues can be identified either by analysing the generation process or the generated RDF data and corrective measures can be taken. In this thesis, one of the objectives is to propose quality assessment and repair techniques that can identify errors in the Linked Data lifecycle and repair them.

The RDF data generated by the aforementioned approaches can be stored either in files as RDF dumps or in other storage mediums such as triple stores. Such data can be accessed through Linked Data portals, SPARQL endpoints, or via Linked Data Fragments servers. Each of those points can be used for data quality assessment. In this thesis, we propose different techniques using such different access points. Furthermore, these techniques explore different dimensions of quality, such as accuracy, consistency, completeness, or timeliness.

\subsection{Main Contributions}

The goal of this thesis is to propose novel and scalable methods for automatic quality assessment and repair of Linked Data. To this end, four main objectives are defined:

The first objective is this thesis is to provide means for representing quality assessment results along with all associated metadata in a comparable manner using well-established standards. To this extent, this thesis has contributed to the advance of the current state of the art by providing:

C1. LDQM, A Linked Data Quality Model for representing Linked Data quality in a standard manner.

The second objective of this thesis is to identify information that could be automatically generated on large-scale datasets and could be used in quality assessment and repair methods. To this extent, this thesis presents new advances by providing the following:

\footnotetext{
${ }^{12}$ https: //researcher. watson. $i b m$. com/researcher/view_group. php?id=2223
} 
C2. Loupe, A framework for Linked Data profiling which consists of an extended dataset description model which captures data profiling metrics and a set of tools for automatically calculating those metrics.

The third objective of this thesis is to propose techniques for automatically deriving Linked Data validation constraints for large datasets. To this extent, this thesis presents new advances by providing the following:

C3. An automatic RDF shape induction method which follows a data-driven approach using Machine Learning algorithms and Linked Data profiling metrics are used as features.

The fourth objective of this thesis is to develop methods for automatic quality assessment and repair. To this extent, this thesis presents new advances by providing the following:

C4. A set of methods for automatic quality assessment and repair using Linked Data profiling metrics and RDF Shapes that includes quality assessment and repair of mapping, repairing inconsistencies, identifying and removing noisy data, and completing type information.

\subsection{Derived Publications}

Parts of the work presented in this Ph.D. thesis were published in various peerreviewed scientific publications belonging to conferences and journals well-recognised and specialised in the research of Semantic Web and Linked Data. This section lists such publications while Table 1.1 shows a summary of the publications related to each of the aforementioned contributions of the thesis.

Table 1.1: A summary of publications

\begin{tabular}{|l|l|}
\hline Contribution & $\begin{array}{l}\text { Related Publications } \\
\text { (listed in Section 1.7) }\end{array}$ \\
\hline C1. Linked Data Quality Model & $\mathrm{J} 3, \mathrm{~W} 8, \mathrm{PD} 3$ \\
\hline C2. Linked Data Profiling Framework & $\mathrm{C} 7, \mathrm{~W} 4, \mathrm{~W} 5, \mathrm{~W} 6, \mathrm{PD} 2, \mathrm{PD} 4$ \\
\hline C3. RDF Shape Induction & $\mathrm{J} 1, \mathrm{~J} 2, \mathrm{C} 1, \mathrm{~W}$, W3 \\
\hline C4. Quality Assessment and Repair Methods & $\begin{array}{l}\mathrm{J} 4, \mathrm{C} 2, \mathrm{C} 3, \mathrm{C} 4, \mathrm{C} 5, \mathrm{C} 6, \mathrm{~W} 1, \\
\mathrm{~W} 5, \mathrm{~W} 7, \mathrm{PD} 1\end{array}$ \\
\hline
\end{tabular}


Chapter 1. Introduction

\section{- Journal publications (JCR)}

J1 M. Rashid, M. Torchiano, G. Rizzo, N. Mihindukulasooriya, O. Corcho, R. García-Castro. A Quality Assessment Approach for Evolving Knowledge Bases. Semantic Web, Volume 10. No 2. January 2019. pp. 349-383.

DOI: https://doi.org/10.3233/SW- 180324

J2 M. Rashid, G. Rizzo, M. Torchiano, N. Mihindukulasooriya, O. Corcho, R. García-Castro. Completeness and Consistency Analysis for Evolving Knowledge Bases. Journal of Web Semantics, Volume 54. January 2019. pp. 48-71.

DOI: https : //doi .org/10.1016/j . websem . 2018.11.00

J3 F. Radulovic, N. Mihindukulasooriya, R. García-Castro, and A. GómezPérez. A Comprehensive Quality Model for Linked Data. Semantic Web Journal. Volume 9. No. 1. November, 2017. pp. 3-24.

DOI: https : //doi . org/10.3233/SW- 170267

J4 N. Mihindukulasooriya, R. García-Castro, M. Esteban-Gutierrez, and A. Gómez-Pérez. A Survey of RESTful Transaction Models: One Model Does not Fit All. Journal of Web Engineering. Volume 15. No. 1. March, 2016. pp. 140-169.

DOI: https://dl.acm.org/doi/abs/10.5555/3177203.3177209

\section{- Conference publications}

C1 N. Mihindukulasooriya, M. Rashid M., G. Rizzo, R. García-Castro, O. Corcho, and M. Torchiano. RDF Shape Induction using Knowledge Base Profiling. In Proceedings of SAC 2018: Symposium on Applied Computing , Pau, France, April 9-13, 2018 (SAC 2018), 8 pages.

DOI: https : //doi .org/10.1145/3167132.3167341

C2 M. Rico, N. Mihindukulasooriya, D. Kontokostas, H. Paulheim., S. Hellmann, and A. Gómez-Pérez. Predicting Incorrect Mappings: A DataDriven Approach Applied to DBpedia. In Proceedings of SAC 2018: Symposium on Applied Computing, Pau, France, April 9-13, 2018 (SAC 2018), 8 pages.

DOI: https : //doi .org/10 . 1145/3167132.3167164

C3 M. Glass, A. Gliozzo, O. Hassanzadeh, N. Mihindukulasooriya, G. Rossiello. Inducing Implicit Relations from Text Using Distantly Supervised Deep Nets. In Proceedings of the 17th International Semantic Web Conference, October 8-12, 2018 (ISWC 2018), pp 38-55.

DOI: https : //doi .org/10 . 1007/978-3-030-00671-6_3 
C4 N. Mihindukulasooriya N., M. Rico, I. Santana-Pérez, R. GarcíaCastro, and A. Gómez-Pérez. Repairing Hidden Links in Linked Data: Enhancing the quality of RDF knowledge graphs. In K-CAP 2017: Knowledge Capture Conference, 2017, Austin, TX, USA. 8 pages.

DOI: https://doi.org/10.1145/3148011.3148020

C5 M. Rico, N. Mihindukulasooriya, and A. Gómez-Pérez. Data-Driven RDF Property Semantic-Equivalence Detection Using NLP Techniques. In the 20th International Conference on Knowledge Engineering and Knowledge Management (EKAW 2016). Bologna, Italy, pp. 797-804.

DOI: https : //doi .org/10. 1007/978-3-319-49004-5_5

C6 N. Mihindukulasooriya, R. García-Castro, A. Gómez-Pérez. A Distributed Transaction Model for Read-Write Linked Data Applications. In the 15th International Conference on Web Engineering. Rotterdam, Netherlands. (ICWE 2015), pp. 631-634.

DOI: https ://doi.org/10 . 1007/978-3-319-19890-3_45

C7 N. Mihindukulasooriya, M. Rico, R. García-Castro, and A. GómezPérez. An Analysis of the Quality Issues of the Properties Available in the Spanish DBpedia. In Conference of the Spanish Association for Artificial Intelligence (CAEPIA 2015), Albecete, Spain. pp. 198-209.

DOI: https : //doi.org/10.1007/978-3-319-24598-0_18

\section{- Workshop publications}

W1 N. Mihindukulasooriya and M. Rico. Type Prediction of RDF Knowledge Graphs using Binary Classifiers with Structural Data. In the $1^{\text {st }} 1$ st International Workshop on Knowledge Graphs on Travel and Tourism in ICWE Workshops and Tutorials. Caceres, Spain. (2018). pp. 1 - 9.

DOI: https : //doi .org/10.1007/978-3-030-03056-8_27

W2 M. Rashid, G. Rizzo, M. Torchiano, N. Mihindukulasooriya, O. Corcho. Knowledge Base Evolution Analysis: A Case Study in the Tourism Domain. In the $1^{\text {st }}$ 1st International Workshop on Knowledge Graphs on Travel and Tourism in ICWE Workshops and Tutorials. Caceres, Spain. (2018). pp. $268-278$.

DOI: https : //doi.org/10.1007/978-3-030-03056-8_26

W3 M. Rashid, G. Rizzo, N. Mihindukulasooriya, M. Torchiano and O. Corcho. KBQ - A Tool for Knowledge Base Profiling and Quality Assessment Using Temporal Analysis. In the $1^{\text {st }}$ International Workshop on Quality Engineering Meets Knowledge Graph (QEKGraph2017) in K-CAP2017 Workshops and Tutorials. Austin, United States. (2017). pp. 
Chapter 1. Introduction

58-63.

DOI: http : //ceur-ws . org/Vol - 2065/paper13.pdf

W4 N. Mihindukulasooriya, R. García-Castro, F. Priyatna, E. Ruckhaus and N. Saturno. A Linked Data Profiling Service for Quality Assessment. In the $4^{\text {th }}$ Workshop on Linked Data Quality co-located with ESWC 2017, (2017), pp. 88-93.

DOI: https : //doi . org/10 . 1007/978-3-319-70407-4_42

W5 N. Mihindukulasooriya, M. Poveda-Villalón, R. García-Castro, and A. Gómez-Pérez. Collaborative Ontology Evolution and Data Quality-An Empirical Analysis. In Proceedings of OWL: Experiences and Directions and Reasoner Evaluation, (2017). pp. 95-114.

DOI: https : //doi . org/10 . 1007/978-3-319-54627-8_8

W6 N. Mihindukulasooriya, M. Poveda-Villalón, R. García-Castro, A. Gómez-Pérez. An RDF Dataset Description Model for Expressing Vocabulary Usage Patterns. In Proceedings of the W3C Smart Descriptions Smarter Vocabularies Workshop. Amsterdam, Netherlands. (2016), pp.2024

DOI: https: //WwW.w3.org/2016/11/sdsvoc/SDSVoc16_paper_7

W7 N. Mihindukulasooriya, G. Rizzo, R. Troncy, O. Corcho, R. GarcíaCastro. A Two-Fold Quality Assurance Approach for Dynamic Knowledge Bases: The 3cixty Use Case. In Proceedings of the $1^{\text {st }}$ International Workshop on Completing and Debugging the Semantic Web co-located with ESWC2016, (2016), pp. 1-12.

DOI: http: //ceur-ws . org/Vol - 1586/codes2.pdf

W8 N. Mihindukulasooriya, R. García-Castro. Describing LDP Applications with the Hydra Core Vocabulary. In Proceedings of the $3^{\text {rd }}$ International Workshop on Services and Applications over Linked APIs and Data co-located with ESWC2015, (2015), pp. 33-36.

DOI: http : / / ceur-ws . org/Vol - 1359/paper5.pdf

\section{- Posters and Demos}

PD1 N. Mihindukulasooriya, O. Hassanzadeh, S. Dash and A. Gliozzo. Towards Comprehensive Noise Detection in Automatically-Created Knowledge Graphs. Demo at the $16^{\text {th }}$ International Semantic Web Conference (ISWC2017), (2017).

DOI: http : / ceur-ws . org/Vol - 1963/paper589.pdf 
PD2 F. Priyatna, E. Ruckhaus, N. Mihindukulasooriya, O. Corcho and N. Saturno MappingPedia: A Collaborative Environment for R2RML Mappings. Demo at $14^{\text {th }}$ Extended Semantic Web Conference (ESWC2017), (2017), pp. 1-4.

DOI: https://doi.org/10.1007/978-3-319-70407-4_22

PD3 N. Mihindukulasooriya, R. García-Castro, A. Gómez-Pérez. LD Sniffer: A Quality Assessment Tool for Measuring Accessibility of Linked Data. Demo in $20^{\text {th }}$ International Conference on Knowledge Engineering and Knowledge Management (EKAW2016), (2016), pp. 1-4.

DOI: https : //doi .org/10 . 1007/978-3-319-58694-6_20

PD4 N. Mihindukulasooriya, M. Poveda-Villalón, R. García-Castro, A. Gómez-Pérez. Loupe-An Online Tool for Inspecting Datasets in the Linked Data Cloud. Demo at the $14^{\text {th }}$ International Semantic Web Conference (ISWC2015), (2015), pp. 1-4.

DOI: http : //ceur-ws .org/Vol - 1486/paper_113.pdf

\subsection{Registered Software}

During this thesis, two software products were created and the corresponding intellectual property rights are enrolled at the regional registry of intellectual property of the Community of Madrid, Spain. Table 1.2 shows the details of the registration.

\begin{tabular}{|l|l|}
\hline & $\begin{array}{l}\text { Loupe, a set of tools for Linked Data profiling. } \\
\text { Application No: M-006795/2018 } \\
\text { Registration No: } 16 / 2019 / 1000 \\
\text { Date: 16/10/2018 } \\
\text { Authority: Registro General de la Propiedad Int- } \\
\text { electual, Comunidad de Madrid }\end{array}$ \\
$\begin{array}{l}\text { LD Sniffer, a tool for evaluating the assessibility } \\
\text { of Linked Data. } \\
\text { Application No: M-006794/2018 } \\
\text { Registration No: } 16 / 2019 / 999 \\
\text { Date: } 16 / 10 / 2018 \\
\text { Authority: Registro General de la Propiedad Int- } \\
\text { electual, Comunidad de Madrid }\end{array}$ \\
\hline
\end{tabular}

Table 1.2: Registered software 
Chapter 1. Introduction

\subsection{Awards}

The work derived from this thesis, contributed to the following awards:

- An FPI grant (BES-2014-068449) from the Spanish Ministry of Education, Culture and Sport (Ministerio de Educación, Cultura y Deporte) funded this thesis. It was associated with the project $4 V$ (TIN2013-46238-C4-2-R).

- $1^{\text {st }}$ place in 2017 Semantic Web Challenge at the $16^{\text {th }}$ International Semantic Web Conference. It consisted of two tasks, fact extraction and fact checking, and the Socrates team, which author was part of, won the first place in both tasks. The author contributed to the solution with data profiling and quality assessment work.

- Best paper award at the $4^{\text {th }}$ Workshop on Linked Data Quality (LDQ 2017) for the publication $W_{4}$.

- Winners of the "Urban Planning and Mobility" category at the $2^{\text {nd }}$ Open Data Hackathon at Cáceres, Spain. The author contributed with data profiling work.

- Finalists and special mention at the Fujitsu Open Data Hackathon for Smart Cities. The author contributed to the project with data profiling work.

\subsection{Research Stays}

During the development of this Ph.D. thesis, a research stay took place at the following institution:

- IBM Research AI at IBM Thomas J. Watson Research Center in New York, USA from the $1^{\text {st }}$ of May 2017 to the $31^{\text {st }}$ of August of 2017. During this research stay, the author worked on detecting quality issues in automatically created knowledge bases, under the supervision of Dr. Alfio Massimiliano Gliozzo (Manager, Knowledge Induction, IBM Watson) and Dr. Oktie Hassanzadeh (Chair, IBM Research Professional Interest Community on Knowledge). The author's work contributed to the system ${ }^{13}$ that won 2017 Semantic Web Challenge. Furthermore, the joint work done led to publication PD1. This research visit was funded by the travel grant (EEBB-I-2017-12726) from the Spanish Ministry of Education, Culture and Sport (Ministerio de Educación, Cultura y Deporte).

\footnotetext{
${ }^{13}$ https : //Www. ibm. com/blogs/research/2017/11/knowledge - base - construction - iswc $2017 /$
} 


\subsection{Work in Research and Innovation Projects and Standardization Bodies}

During the development of this thesis, the author has participated in the following research and innovation projects contributing to the tasks related to the thesis:

- Datos 4.0: Challenges and Solutions (TIN2016-78011-C4-4-R), a project aimed at addressing the challenges of the fourth revolution in data management, which is characterised not only by the huge volume of data, but also of the complexity and heterogeneous nature and, above all, by demanding usage scenarios which need the efficient production of useful and quality knowledge, obtained from the raw data, using from linked open data to sophisticated exploitation processes. The Linked Data Quality Model (C1), Linked Data Profiling Framework (C2), RDF Shape Induction method (C3), and Linked Data quality assessment and repair techniques (C4) provide valuable contributions to develop solutions to the challenges identified in this project.

- 4V: Volumen, Velocidad, Variedad y Validez en la gestin innovadora de datos (TIN2013-46238-C4-2-R), a project aimed at providing techniques for innovative management of big data characterised by volume, velocity, variety, and validity. One of the main objectives of the project was to provide novel techniques for quality assessment and repair of Linked Data. The Linked Data Quality Model (C1) developed in this thesis was used in this project.

- Application of the IBM Watson Ecosystem for providing Technology Intelligence services to Small and Medium Enterprises (IBM Shared University Research), a project aimed at designing and implementing an innovative technology intelligence service for technology-based small and medium enterprises (SMEs) based on the IBM Watson system.

- LIDER (FP7-610782), a project that proposed Linked Data as an enabler of cross-media and multilingual content analytics for enterprises across Europe. Linked Data profiling techniques that are developed as the second contribution of the thesis (C2) provided valuable information for content analytics.

- ALM iStack, a project aimed at developing an open-source Application Lifecycle Management platform that automates and supports the whole software development lifecycle by integrating the best existing open-source tools.

Further, during this thesis, the author participated in several W3C Working Groups providing feedback based on the work on this thesis. Main contributions include: 
- Dataset Exchange Working Group (DXWG). The DXWG is charted with the objective of revising the Data Catalog Vocabulary, DCAT, taking account of related vocabularies and the extensive work done in developing a number of its application profiles. The proposed DCAT 1.1 vocabulary $^{14}$ is related to the data profiling and quality aspects of the work presented in this thesis (C2), and the author actively participated in the group discussions and weekly teleconference providing feedback and submitting use cases and requirements ${ }^{15}$ based on the Linked Data profiling use cases and tasks that are discussed in (Chapter 5).

- Data on the Web Best Practices Working Group (DWBP WG). The DWBP WG was chartered with the objective of developing the open data ecosystem facilitating better communication between developers and publishers; providing guidance to publishers that will improve consistency in the way data is managed, thus promoting the re-use of data; and fostering trust in the data among developers. In this context, one of the deliverables of the working group was Data Quality Vocabulary $(D Q V)^{16}$ which is a vocabulary for describing the quality of a dataset, either by the dataset publisher or by a broader community of users. The author of the thesis significantly contributed to the vocabulary based on analysis of Linked Data quality issues that are discussed in this thesis. Furthermore, the Linked Data Quality Model presented in this thesis (Chapter 4) extends DQV with Linked Data specific aspects.

- Linked Data Platform Working Group (LDP WP). The LDP WG was chartered with the objective of producing a W3C Recommendation for HTTPbased (RESTful) application integration patterns using read/write Linked Data. In the context of enterprise read-write Linked Data applications, the validation of RDF data that are produced and consumed by the applications plays a key role. The IBM's submission of the OSLC Resource Shapes[209] specification which eventually led to the W3C Shapes Constraint Language (SHACL) [137] specification was motivated by the LDP use cases. The author of this thesis participated actively in the LDP WG and authored the documents: Linked W3C Data Platform 1.0 Primer [47] and Linked Data Platform Best Practices and Guidelines [160].

\footnotetext{
${ }^{14}$ https : //Www.w3.org/TR/vocab-dcat - 2/

${ }^{15}$ https://WWw.w3.org/TR/dcat-ucr/\#ID4

${ }^{16}$ https://www.w3 . org/TR/vocab-dqv/
} 


\subsection{Structure of the Document}

The rest of this document is organised as follows:

- Chapter 1 provides an introduction to the thesis and a summary of the contributions of the thesis, associated research stays, and the relations to research and innovation projects and standardisation initiatives.

- Chapter 2 analyses the state of the art in topics related to the research questions of the thesis.

- Chapter 3 enumerates the open research problems identified in the state of the art, presents the objectives and contributions of the thesis, and defines the assumptions, hypotheses and restrictions. Furthermore, this chapter also describes the research methodology followed in the development of the thesis.

- Chapter 4 presents LDQM, a comprehensive quality model for Linked Data, and provides a set of metrics for assessing Linked Data quality and the methodology that was used for developing the model. This chapter covers the first objective (O1) of the thesis and its corresponding contributions (C1).

- Chapter 5 presents a framework for Linked Data profiling including a set of Linked Data profiling tasks, a tool suite for Linked Data profiling, and an extended dataset description model for representing profiling metrics. This chapter covers the second objective (O2) of the thesis and its corresponding contributions (C2).

- Chapter 6 presents a method for RDF Shape Induction using Linked Data profiling metrics. This chapter covers the third objective (O3) of the thesis and its corresponding contributions (C3).

- Chapter 7 presents a set of Linked Data quality assessment and repair methods using Linked Data profiling metrics and RDF Shapes. This chapter covers the fourth objective $\left(\mathrm{O}_{4}\right)$ of the thesis and its corresponding contributions $\left(\mathrm{C}_{4}\right)$.

- Chapter 8 presents the evaluation, in which, the contributions of Chapter 4 to 7 are evaluated.

- Chapter 9 describes several use cases where the contributions of the thesis were used, draws some conclusions and presents ideas for future work. 


\section{Chapter 2}

\section{State of the Art}

The work presented in thesis focuses on proposing novel methods and techniques for automatic quality assessment and repair of Linked Data. This chapter presents the existing work that is related to the topic of this thesis, with the purpose of providing an overview of the current state of the art in the research areas related to this thesis and of identifying the open research problems in those research areas. Because the work proposed in thesis is guided by four objectives as it will be discussed in Chapter 3, we cover a wide range of related work related to the next chapters of the thesis in different sections of the thesis and identify the limitations with respect to the objectives of this thesis.

Section 2.1 discusses the work related to the Linked Data Quality Model; Section 2.2 discusses the work related to data profiling; Section 2.3 discusses the work related to RDF Shape Induction. Finally, Section 2.5 presents the conclusions of the analysis of state of the art.

\subsection{Linked Data Quality Model}

Data quality dimensions and metrics In the Linked Data research literature, a large number of quality dimensions and metrics for assessing Linked Data are proposed. These dimensions and metrics are quite diverse since different authors focus on different aspects of Linked Data, i.e., on different characteristics (e.g., trust, completeness, accuracy) and on different measures for these characteristics (e.g., missing links, an indication of attribution, semantically incorrect values). Furthermore, some authors have developed methodologies and tools for Linked Data evaluation, which are also characterized by high diversity in evaluated dimensions and metrics.

Gamble and Goble [89] focus on evaluating trust of Linked Data datasets. Their approach is based on decision networks which allow modelling relationships between different variables based on probabilistic models. Furthermore, they discuss several 
dimensions of data quality:

- Quality dimension, which is assessed against some quality standard and which intends to provide specific measures of quality

- Trust dimension, which is assessed independent of any standard and is intended to asses the reputation

- Utility dimension, which intends to assess whether the data fit the purpose and satisfy the needs of users

Gil and Arts [95] focus their work on the reputation (trust) of web resources. The main sources of trust assessment according to these authors are direct experience and user opinions, which are expressed through reliability (based on credentials and performance of the resources) and credibility (users view of the truthfulness of information). The trust is represented with a web of trust, where nodes represent entities and edges are trust metrics that one entity has towards the other. Furthermore, the authors identified a number of factors that influence user opinion of trust. Those factors include topic, context and criticality, popularity, authority, experience, recommendations from others, relation to other resources, provenance, user expertise, bias, incentive, existence of limited resources, agreement, specificity, the likelihood of correctness of the content, age, appearance, deception, and recency.

Golbeck and Mannes [98] focus on trust in networks and their approach is based on the interchange of trust, provenance, and annotations. They have developed an algorithm for inferring trust and for computing personal recommendations using the provenance of already defined trust annotations. Furthermore, they apply the algorithm in two examples to compute the recommendations of movies and intelligent information.

Bonatti et al. [39] focus on data trust based on annotations. They identify several annotation dimensions:

- Blacklisting, which is based on noise, on void values for inverse functional properties, and on errors in values

- Authoritativeness, which is based on cross-defined core terms that can change the inferences over those terms that are mandated by some authority (e.g., owl:Thing), and that can lead to the creation of irrelevant data

- Linking, which is based on determining the existence of links from and to a source in a graph, with a premise that a source with a higher number of links is more trustworthy and is characterized by higher quality of the data 
Chapter 2. State of the Art

Chen and Garcia [50] focus on quality assessment through association mining. They particularly focus on the appropriateness of the amount of data and on the coherence of data attributes. Furthermore, they propose a metric for amount of data, which is a ratio between a number of semantically valid rules to a number of non-trivial rules.

Furber and Hepp [86] focus on the assessment of accuracy, which include both syntactic and semantic accuracy, timeliness, completeness, and uniqueness. One measure of accuracy consists of determining inaccurate values using functional dependence rules, while timeliness is measured with time validity intervals of instances and their expiry dates. Completeness deals with the assessment of the completeness of schema (representation of ontology elements), completeness of properties (represented by mandatory property and literal value rules), and completeness of population (representation of real world entities). Uniqueness refers to the assessment of redundancy, i.e., of duplicated instances.

Hogan et al. [111] focus their work in assessment of mainly errors, noise and modelling issues. Measures that they propose include:

1. Use of blank nodes

2. Use of HTTP URIs

3. Use of short URIs

4. Use of stable URIs

5. Existence of external links

6. Use of dereferencible links

7. Existence of machine readable license

8. Existence of human readable license

9. Reuse of well-known vocabulary terms

10. Use of prolix RDF features (reification, containers and collectors)

Jacobi et al. [120] develop a framework for the assessment of trust. Their framework is based on the use of trust ontologies in order to assign a trust value to data sources or rules and provenance ontologies in order to capture the data generation. The assessment of trust is then used for transferring trust from known to unknown data.

Lei et al. [148] focus on several types of quality problems related to accuracy. In particular, they evaluate: 
1. Incompleteness (objects in a data source are not exhaustive compared to real world)

2. Existence of duplicate instances that refer to the same object (redundancy)

3. Ambiguity (mapping an instance in a data source to more than one real world object)

4. Inaccuracy of instance labels and inaccuracy of classification of instances (assigning as a type a class in a higher position in a hierarchy)

Shekarpour and Katebi [212] focus on assessment of trust of a data source. They first discuss several models of trust (centralized model, distributed model, global model and local model), and then they develop a model for assessment of trust of a data source which is based on a propagation of trust assessment from data source to triples, and then aggregation of all the triple assessments.

Rula et al. [207] start from the premise of dynamicity of Linked Data and focus on assessment of timeliness in order to reduce errors related to outdated data. To measure timeliness, they define a currency metric which is calculated in terms of differences between the time of the observation of data (current time) and the time when the data was modified for the last time. Furthermore, they also take into account the difference between the time of the observation of the data and the time of data creation.

Hartig [106] developed a model for assessment of the trustworthiness of RDF statements based on the belief in truthfulness of these statements. He defined a trust function that assigns a subjective trust value to a statement, and a trust aggregation function that determines the trust of a set of RDF statements. Trust function developed by Hartig relies on several different techniques:

1. User-based rating, in which users can give ratings related to trust

2. Provenance-based technique, which is related to the provenance of the data

3. Opinion-based technique, which aggregates trust ratings from different consumers and reliability of these consumers

The trust ratings are defined as a number in the range $[-1,1]$, where -1 denotes total disbelief, and 1 denotes total belief in trustfulness of the RDF statements.

Gil and Ratnakar[96] focus on the assessment of trust. Their work consists of assigning user ratings for each source in terms of reliability and credibility. The rating that can be assigned is denoted by a number in the range $[1,6]$, where 6 denotes the highest reliability/credibility. Furthermore, they define ordinal rating in terms of whether a user has used particular statement in reaching a conclusion in a given task. 
The overall trust is then computed as an aggregation of reliability, credibility, and previously mentioned ordinal ratings.

Flemming [81] focuses on a number of measures for assessment the quality of Linked Data. These measures include:

1. Availability of data

2. Accessibility of the SPARQL endpoint and of the server, as well as of the RDF dump

3. Scalability, throughput and latency of a data source

4. Licensing, which include existence of machine-readable and human-readable license statement

5. Existence of a permission to use the data, as well as attribution

6. Correct usage of URI types and existence of dead links

7. Existence of provenance information

8. Number of triples, number of instances and number of properties

9. Frequency of change and existence of outdated data

10. Authenticity of the publisher

11. Reuse of existing vocabularies

12. Human-readable labeling of instances

13. Indication of vocabularies used in a dataset

14. Provision of the data in various languages

15. Existence of different ways of accessing the data

16. Application of content negotiation of published dataset

Hogan et al. [110] provide a framework for assessment of Linked Data which, similar as in the case of Flemming [21], describes the quality in terms of various metrics. Their work includes the quality assessment in terms of:

- Availability of structured data

- Accessibility of SPARQL endpoint and of the server 
- Accessibility to RDF dumps

- Errors in dereferencibility

- Correctness of the format of datatype literals

- Compatibility of literals with datatypes

- Usage of undefined classes or properties

- Respect of vocabulary constraints

- Population completeness, which they define as a ratio between a number of represented real-world objects and a number of real-world objects

- Usage of collections, containers and reification

Gueret et al. [101] define a set of network measures for assessment of Linked Data mappings. These measures are:

- Degree, which is calculated in terms of a number of incoming and outcoming edges

- Clustering coefficient, which is calculated in terms of a ratio of a number of links of node neighbors and a total possible number of links

- Centrality, which is calculated in terms of a number of connections the node takes part in

- SameAs chains, which is calculated in terms of a number of sameAs chains that are not closed

- Descriptive richness, which is calculated in terms of the number of new edges brought to a resource through the sameAs relations

Mendes et al. [157] developed a framework for Linked Data quality assessment. One of the contexts in quality assessment in their work is the existence of conflict between values in different data sources. Furthermore, they use a set of measures for Linked Data quality assessment, which include:

- Intensional completeness, which is calculated in terms of a ratio between a number of unique attributes of a dataset and a total number of attributes in a universe of attributes

- Extensional completeness, which is calculated in terms of a ratio between a number of unique objects in a dataset and a total number of objects in a universe of objects 
Chapter 2. State of the Art

- Recency and reputation

- Time since data modification

- Property completeness, which is calculated in terms of a ratio between a number of properties in a dataset and a total number of properties in a universe of properties

- Property conciseness, which is calculated in terms of a ratio between a number of objects with a unique value for specific property and a total number of unique objects in a dataset with that specific property

- Property consistency, which is calculated in terms of a ratio between a number of objects in a dataset without conflicts for a particular property, and a total number of objects in a dataset with that particular property

Kontokostas et al. [142] developed a test-driven evaluation of Linked Data quality in which they focus on coverage and errors. The measures they use are the following:

- Property domain coverage

- Property range coverage

- Class instance coverage

- Missing data

- Mistypes

- Correctness of the data

Knuth et al. [139] in their work identify the key challenges for Linked Data quality. As one of the key factors for Linked Data quality they outline validation which, in their opinion, has to be integral part of Linked Data lifecycle. Additional factor for Linked Data quality is version management, which can create problems in provenance and tracking. Finally, as another important factor they outline the usage of popular vocabularies or manual creating of new correct vocabularies.

Emburi at al. [67] developed a framework for automatic crawling the Linked Data datasets and improving dataset quality. In their work, the quality is focused on errors in data and the purpose of developed framework is to correct errors.

Although there is a significant effort in the Semantic Web community to evaluate the quality of Linked Data and related datasets, in the current state of the art there is no quality model for Linked Data that would provide a unique reference for researchers and practitioners. This leads to difficulties in assessing quality of datasets and performing benchmarking and comparisons of datasets that describes the same or similar domains. 


\subsection{Linked Data Profiling}

The initial works of quality metrics and dimensions are mainly focused on trust quality dimensions. This initial focus on trust is understandable as the generation of Linked Data is done in a distributed manner involving many parties including many data producers and consumers. However, later on these dimensions expanded covering variety of dimensions such as completeness, accuracy, timeliness, or accessibility.

The more recent work on quality dimensions and metrics focus on inherent characteristics of RDF such as property conciseness [157], property consistency [157], property domain coverage, property range coverage, etc. Analysing the patterns of vocabulary usage in data can provide valuable insights in assessing quality of Linked Data along those dimensions. One of the techniques to analyse the vocabulary usage is data profiling. Data profiling [178] provides methods for examining the data available in an existing data source and collecting statistics and information about that data. Such information can be used to understand the data and to detect irregularities, for instance, detection of frequent value patterns or validating the uniqueness of given set of values.

Böhm et al. [37] developed ProLoD, a set of methods for profiling Linked Data in three different levels: (i) domain level, (ii) schema level, and (iii) data level. In domain level, clustering techniques are used to partition the datasets into semantically correlated smaller subsets and label them applying techniques such as term frequencyinverse document frequency, (tf-idf) on the textual properties, for instance, labels and comments. These smaller partitions of datasets enable better profiling. In schema level, several steps are taken for finding equivalent properties, discovering properties with large number of missing values, and finding property correlations using techniques such as association rule mining. In data level, value distributions are analysed for different data types for detecting irregularities. The authors of ProLoD have used these methods to analyse DBpedia infobox dataset.

Auer et al. [69] developed LODStats, an approach for gathering statistical dataset characteristics using 32 schema level statistical criteria. LODStats is implemented following a statement-stream-based approach for having a smaller memory footprint and better performance during the dataset analysis. However, this statement-streambased approach puts limitations on the type of metrics that can be gathered as more complex criteria that involves joining multiple statements can not performed. The metadata generated by LODStats can be used to perform quality analysis of RDF datasets with regard to the used vocabularies, properties, domain and range constraints of properties.

Palmonari et al. [183] developed ABSTAT, a framework for generating Linked Data summaries as statistics about triple patterns using an ontology-driven data abstraction model. ABSTAT extracts summaries that are represented in RDF and can 
Chapter 2. State of the Art

be queried or navigated through a web interface. It uses Abstract Knowledge Patterns (AKPs) which are triples having the form of < subjectType,pred,objectType $>$, which represent the occurrence of triples $<$ sub,pred,obj $>$ in the data, such that subjectType is a minimal type of sub and objectType is a minimal type of obj. Though the ABSTAT framework is currently only used for data understanding and navigation.

\subsection{RDF Shape Induction}

Ontology Learning (OL) is commonly defined as a field that comprises techniques for automated acquisition of ontological knowledge from data. Thus, the paradigm has shifted such that many approaches do not aim to generate a full fledged, gold-standard ontology from data anymore, but they rather focus on acquiring axioms of certain shapes such as concept definitions, atomic subsumptions, disjointness axioms. There are several works done on induction of Description Logic axioms using methods, such as,

Association rule mining (ARM): Abedjan [1] et al. present rule-based approaches for predicate suggestion, data enrichment, ontology improvement, and query relaxation. They identified inconsistencies in the data through predicate suggestion, enrichment with missing facts, and alignment of the corresponding ontology. Also they provide users to handle inconsistencies during query formulation through predicate expansion techniques.

Probabilistic graphical models (PGMs): An approach of probabilistic graphical models (PGMs) allow to generate interpretable models that are constructed and then manipulated by reasoning algorithms [140]. These models can also be learned automatically from data, allowing the approach to be used in cases where the manual building of a model is difficult or even impossible.

Statistical Relational Learning (SRL) It is a branch of machine learning that tries to model a joint distribution over relational data [94]. SRL is a combination of statistical learning which addresses uncertainty in data and relational learning which deals with complex relational structures [134].

Inductive logic programming (ILP) Buhmann et al. [45] present an approach of inductive lexical learning of class expression by combining an existing logical learning approach with statistical relevance measures applied on textual resources.

Pattern extraction is an area of work where RDF data is analyzed to extract common patterns, for example, in the form of Frequent Graph Patterns [22] or 
Statistical Knowledge Patterns [36]. These approaches analyze the underlying RDF data and extract the characteristics related to the ontological axioms based on most frequent patterns. This approach is closely related to the work presented in this paper.

Our final goal is different from these research approaches. Instead of inducting the ontological axioms, our goal is to induct validation rules. In recent years various RDF validation languages have been introduced based on expressing constraints.

- The Web Ontology Language (OWL) [211] is an expressive ontology language based on Description Logics (DL). The semantics of OWL addresses distributed knowledge representation scenarios where complete knowledge about the domain cannot be assumed. To address the above mismatch, some approaches use OWL expressions with Closed World Assumption and a weak Unique Name Assumption so that OWL expressions can be used for validation purposes such as Stardog ICV ${ }^{1}$ and Tao et al. [219].

- The W3C Shapes Constraint Language (SHACL) [137] is used represent constraints for validating RDF graphs against a set of conditions. These conditions are provided as shapes and other constructs expressed in the form of an RDF graph. In particular it helps to identify constraints by using SPARQL. Also, it provides a high level vocabulary to identify predicates and their associated cardinalities, and datatypes.

- The Shape Expressions (ShEx) [194] language describes RDF nodes and graph structures. A node constraint describes an RDF node (IRI, blank node or literal) and a shape describes the triples involving nodes in an RDF graph. These descriptions identify predicates and their associated cardinalities and datatypes.

- ASHACL: Alternative Shapes Constraint Language [185] is a variant of the W3C Shapes Constraint Language, is designed to determine whether an RDF graph meets some conditions.

- SPARQL Inferring Notation (SPIN) ${ }^{2}$ [138] constraints associate RDF types or nodes with validation rules. In particular it allows users to use SPARQL to specify rules and logical constraints.

In particular, these shape expression languages, namely, ShEx, SHACL, and SPIN, aim to validate RDF data and to communicate data semantics among users. They do

\footnotetext{
${ }^{1}$ https://www.stardog.com/docs/

${ }^{2}$ http://spinrdf.org/
} 
Chapter 2. State of the Art

cover constraints such as keys and cardinality; however, their expressivity is limited and require user interventions in every step. In our approach we aim to automate the process of shape generation using ML. More specifically various constraints can be envisioned in RDF based on their success in the relational model, such as cardinality and range constraints.

Cardinality estimation has been studied in many different domains including relational data. In addition to integrity constraint validation, it has many other applications such as network monitoring for detecting DDoS attacks or worm propagation, link-based spam detection, relation join query optimizations.

The existing cardinality estimation algorithms such as Hit Counting [78], Adaptive Sampling [77], Probabilistic Counting [232], HYPERLOGLOG [108] aim to estimate the number of distinct elements in very large set of data with duplicate elements. Neuman and Moerkotte have proposed "characteristic sets" for performing cardinality estimations for RDF queries with multiple joins [180]. A more recent work from Boneva et al. $[40,41]$ provides a designer for creating ShEx and SHACL Constraints.

These works differ from the work presented in this paper on two axes. First, they are focused on determining the cardinalities of each value rather than the cardinality of the entity-value relation. Second, they are focused on query optimization than integrity constraint validation. However, we use the base of these works such as analysis of mean, variance, and other statistical features to derive an approach for cardinality estimation for integrity constraint validation.

\subsection{Methods for Linked Data Quality Assessment and Repair}

\subsubsection{Linked Data Quality Assessment Tools}

Table 2.1 illustrates a list of fourteen quality assessment tools found in literature with their year of publication and a brief description of their capabilities.

These tools use the quality dimensions and metrics defined in the studies presented in the Linked Data Quality Model section to evaluate Linked Data and provide quality indicators. While some of them are focused on one quality aspect, some others are more generic frameworks that cover a wide range of quality dimensions. 
Table 2.1: Linked Data quality assessment tools

\begin{tabular}{|c|c|c|}
\hline Tool & Year & Description \\
\hline Trellis [96] & 2002 & $\begin{array}{l}\text { A system that helps users annotate their analysis } \\
\text { of alternative information sources that can be con- } \\
\text { tradictory and incomplete. As the user makes a } \\
\text { decision on which sources to dismiss and which to } \\
\text { believe in making a final decision, TRELLIS cap- } \\
\text { tures the derivation of the decision in a semantic } \\
\text { markup. TRELLIS then uses these annotations to } \\
\text { derive an assessment of the source based on the } \\
\text { annotations of many individuals. }\end{array}$ \\
\hline TrustBot [99] & 2003 & $\begin{array}{l}\text { An IRC bot that makes trust recommendations to } \\
\text { users (based on the trust network it builds), the } \\
\text { users have the flexibility to submit their own URIs } \\
\text { to the bot at any time while incorporating the data } \\
\text { into a graph. }\end{array}$ \\
\hline tSPARQL [106] & 2008 & $\begin{array}{l}\text { A trust-aware SPARQL extension that adds two } \\
\text { new clauses, namely the TRUST AS clause and the } \\
\text { ENSURE TRUST clause. The first clause allows } \\
\text { access to the trust value associated to the triples } \\
\text { that match a specific graph pattern; the second } \\
\text { clause expresses a trust requirement. }\end{array}$ \\
\hline WIQA [32] & 2009 & $\begin{array}{l}\text { A set of software components for filtering infor- } \\
\text { mation using different quality-based information } \\
\text { filtering policies. The WIQA framework can be em- } \\
\text { ployed by applications which process information } \\
\text { of uncertain quality and want to enable users to } \\
\text { filter information using different policies. }\end{array}$ \\
\hline ProLOD [37] & 2010 & $\begin{array}{l}\text { A web-based profiling tool, which allows you to an- } \\
\text { alyze Linked Open Data (LOD) and thus helps you } \\
\text { to gain a deeper understanding of the underlying } \\
\text { structure and semantics. }\end{array}$ \\
\hline
\end{tabular}

Table 2.1 - Continued on next page 
Chapter 2. State of the Art

Table 2.1 - Continued from previous page

\begin{tabular}{|c|c|c|}
\hline Tool & Year & Description \\
\hline LINK-QA [101] & 2012 & $\begin{array}{l}\text { A framework for assessing the quality of the Web } \\
\text { of Data through the analysis of its constituent } \\
\text { parts., Given that the Web of Data is a network, } \\
\text { its global properties can be assessed using network } \\
\text { measures. These statistical techniques provide sum- } \\
\text { maries of the network along different dimensions. } \\
\text { These dimensions can be used to get an overall } \\
\text { perspective on the quality of the network. }\end{array}$ \\
\hline Sieve [157] & 2012 & $\begin{array}{l}\text { A framework that allows Web data to be filtered } \\
\text { according to different data quality assessment poli- } \\
\text { cies and provides for fusing Web data according to } \\
\text { different conflict resolution methods. }\end{array}$ \\
\hline Triple CheckMate [236] & 2013 & $\begin{array}{l}\text { A tool wherein a user assesses an individual re- } \\
\text { source and evaluates each fact for correctness. The } \\
\text { tool follows a two phase process. The first phase } \\
\text { includes the detection of common quality problems } \\
\text { and their representation in a quality problem tax- } \\
\text { onomy. In the manual process, the second phase } \\
\text { comprises of the evaluation of a large number of } \\
\text { individual resources, according to the quality prob- } \\
\text { lem taxonomy via crowdsourcing. }\end{array}$ \\
\hline LiQuate [206] & 2013 & $\begin{array}{l}\text { A tool that relies on Bayesian Networks to identify } \\
\text { potential quality problems and ambiguities among } \\
\text { data and links. It relies on statistical reasoning to } \\
\text { analyze the quality of data based on completeness } \\
\text { and potential redundancies or inconsistencies. }\end{array}$ \\
\hline LODStats [69] & 2013 & $\begin{array}{l}\text { A statement-stream-based approach for gathering } \\
\text { comprehensive statistics about datasets adhering } \\
\text { to the Resource Description Framework (RDF). } \\
\text { LODStats is based on the declarative description } \\
\text { of statistical dataset characteristics. }\end{array}$ \\
\hline
\end{tabular}

Table 2.1 - Continued on next page 
Table 2.1 - Continued from previous page

\begin{tabular}{|l|l|l|}
\hline Tool & Year & Description \\
\hline RDFUnit [142] & 2014 & $\begin{array}{l}\text { A test driven data-debugging framework that can } \\
\text { run automatically generated (based on a schema) } \\
\text { and manually generated test cases against an end- } \\
\text { point. All test cases are executed as SPARQL } \\
\text { queries using a pattern-based transformation ap- } \\
\text { proach. }\end{array}$ \\
\hline DaCura [74] & 2014 & $\begin{array}{l}\text { A test driven data-debugging framework that can } \\
\text { run automatically generated (based on a schema) } \\
\text { and manually generated test cases against an end- } \\
\text { point. All test cases are executed as SPARQL } \\
\text { queries using a pattern-based transformation ap- } \\
\text { proach. }\end{array}$ \\
\hline ABSTAT [183] & 2015 & $\begin{array}{l}\text { A framework that extracts summaries of linked } \\
\text { datasets based on an ontology-driven data abstrac- } \\
\text { tion model making it easier to identify quality is- } \\
\text { sues. }\end{array}$ \\
\hline Luzzu [57] & 2016 & $\begin{array}{l}\text { A Quality Assessment Framework for Linked Open } \\
\text { Datasets. It is a generic framework based on the } \\
\text { Dataset Quality Ontology (daQ), allowing users to } \\
\text { define their own quality metrics. }\end{array}$ \\
\hline
\end{tabular}

\subsubsection{Linked Data Repair Tools}

Though there are plenty of tools available in literature for quality assessment, there are not many tools available for repairing low quality datasets. The repair tools found in literature are mostly focused on the TBox or the ontologies.

Ji et al.[124] has developed, RaDON, a system for diagnosing and repairing ontologies by extending the abilities of the state-of-the-art reasoners. They are mainly focused on inconsistencies that occur when integrating multiple distributed ontologies. Similarly, Hellmann et al. [107] describe how to use the Ontology Repair and Enrichment ${ }^{3}$ (ORE) tool for fixing inconsistencies in OWL ontologies. It is mainly focused on the schema or the TBox of data, and provide capabilities related to ontology debugging and enrichment. In this thesis, our main focus of quality assessment and repair of large scale data mainly focusing on instance or $A B o x$ data.

Beek et al. [24] have developed, LOD Laundromat, a system that cleans datasets and publishes a cleaner version of the data. LOD Laundromat is mainly focused on

\footnotetext{
${ }^{3}$ https://github.com/SmartDataAnalytics/ORE
} 
two aspects: removing duplicate triples and eliminating syntax errors. While such tasks help to significantly clean the datasets and make them processed by machines more efficiently, there are other aspects of data that has to be considered as we discuss in detail in Chapter 4. In this thesis, we focus primarily on repairing inconsistencies found in data.

\subsection{Conclusions}

Linked Data Quality Model: Section 2.1 shows that diverse quality dimensions and metrics are defined to measure the same or similar quality characteristics in the literature. Those dimensions and metrics are specified using varying terminology and definitions in different studies and tools. Quality models provide a good basis for providing consistent terminology and guidance for quality assessment, nevertheless, we did not find Linked Data quality models that follow established standards for data quality such as ISO 25012 from International Organization for Standardization (ISO).

Linked Data Profiling: In Section 2.2, we analyzed the current Linked Data profiling methods and tools. As we discussed, they are mostly focused on coarsegrained profiling metrics of Linked Data. In this thesis, we argue that more fine-grained metrics are needed for quality assessment and repair use cases. For instance, most of the models in the literature do no cover network metrics of the RDF graph (e.g., in-degrees, out-degrees, components) or value distribution statistics (e.g., min, max, mean, standard deviation of numeric values or string lengths).

RDF Shape Induction: In Section 2.3, we looked at approaches for generic ontology learning that provide background for $R D F$ Shape induction. Nevertheless, these approaches were mainly focused on learning or inducing the ontology, and not validation constraints.

To the best of author's knowledge, there were no existing approaches for inducting RDF Shapes-based on Linked Data profiling at the time of writing this thesis. Because Shapes Constraint Language (SHACL) is a relatively new standard published in July 2017, we believe that more approaches will follow in the future.

Quality Assessment and Repair Methods: In Section 2.4, we analyzed the existing quality assessment and repair tools for Linked Data. By the time of writing this thesis, the author has not found approaches for Linked Data quality assessment and repair focused Linked Data profiling and RDF Shape Induction though there are 
approaches, though there are some approaches that use metrics extracted from data as ad-hoc features.

Nevertheless, we have seen that data profiling is being widely used in other domains such as relational databases for extracting valuable information for quality assessment and repair. Furthermore, while analysing the techniques for Linked Data quality assessment and repair we have noticed that there is a lack of methods for repairing Linked Data. 


\section{Chapter 3}

\section{Work Objectives}

This chapter provides a high-level overview of the thesis, discusses its main objectives (Section 3.1) and the open research problems it aims to address (Section 3.2), together with its main contributions (Section 3.3). Furthermore, the chapter also presents the work assumptions, hypotheses, and restrictions of the thesis (Section 3.4), as well as the plan for the evaluation of the stated hypotheses (Section 3.5). Finally, it presents the research methodology and research process followed while conducting the research (Section 3.6).

\subsection{Thesis Objectives}

The general objective of this thesis is to propose novel and scalable methods for automatic quality assessment and repair of Linked Data. These methods cover both the datasets, which are the outcome of Linked Data generation process, as well as other intermediate artefacts such as mappings that are used to generate RDF. The motivation for proposing automatic methods and techniques is to significantly reduce the manual effort required in quality assessment and repair tasks in the current Linked Data quality approaches, thus making such techniques suitable for large-scale Linked Data sources containing millions of triples such as DBpedia.

To this end, four main objectives are defined:

- O1: The first objective of the thesis is to provide means for representing quality assessment results along with all associated metadata in a comparable manner using well-established standards. More concretely, to propose a quality model for Linked Data quality specification, adhering to the well-established ISO standard (ISO 25012) for data quality and the W3C Data Quality Vocabulary so that the quality assessment results about datasets can be published in a standard and interoperable. By using a common quality 
model, Linked Data quality assessment results produced by many different parties and tools will be comparable. Furthermore, such models will make it possible to measure and describe the improvements made by repair techniques in a standard manner.

- O2: The second objective is to identify information that could be automatically generated on large-scale datasets and could be used for quality assessment and repair tasks. This objective is planned to be achieved by improving RDF data profiling techniques focusing on the Linked Data quality assessment and repair use cases and by extending the current RDF data profiling techniques to include fine-grained profiling information that is needed for quality assessment. Once the fine-grained metrics useful for data quality are identified, an RDF dataset description model can be developed to represent data profiling metrics, dataset statistics, and other data pattern information with a sufficient level of detail. Such data profiling techniques and dataset description models will enable the use of data profiling information in Linked Data quality assessment and repair.

- O3: The third objective is to propose methods for automatically deriving Linked Data validation constraints for large datasets. This objective is planned to be achieved by developing an automatic RDF Shape Induction method to generate constraints using a data-driven approach without requiring manual effort and by combining the aforementioned constraints into RDF Shapes. Data validation constraints and RDF Shapes are induced using machine learning techniques over data profiling metrics. These RDF Shapes will provide the necessary inputs for automatic data quality assessment and repair techniques.

- O4: The fourth objective is to develop methods and techniques for automatic quality assessment and repair using integrity constraints represented as RDF Shapes. Such methods and techniques will reduce the manual effort required for quality assessment and repair and will enable efficient quality assessment and repair of large-scale Linked Data datasets such as DBpedia. Because it is not possible to develop methods to cover all quality dimensions within the duration of the thesis, we plan to select four specific use cases and develop techniques for quality assessment and repair for those. When selecting these use cases, we plan to consider a variety of factors such as the generation process (e.g., mapping-based, open information extraction), what is being assessed (e.g., RDF output, quality of the mappings), dataset characteristics (e.g., public/enterprise, size), and finally quality dimensions covered (e.g., consistency, accuracy, completeness). We plan to select diverse use cases to cover different combinations of those. 


\subsection{Open Research Problems}

In this section, we describe the open research problems for achieving each of the thesis objectives outlined in the previous section. We have identified several open research problems both from a methodological perspective and from a technological perspective.

In order to achieve the first objective, from a methodological perspective, the following list of open research problems must be solved:

- The literature on Linked Data quality during the last decade (as discussed in Section 2.1) shows that diverse quality dimensions and metrics are defined to measure the same or similar quality characteristics. Those dimensions and metrics are specified using varying terminology and definitions in different studies and tools. As a consequence, Linked Data evaluation results are not comparable between different studies or interoperable between multiple tools. For instance, this makes it difficult to compare the results reported by several data quality assessment or repair algorithms or to select the right data source according to a set of given quality needs. Quality models provide a good basis for providing consistent terminology and guidance for quality assessment and are the basis for the evaluation of any product or service. Nevertheless, currently there is a lack of a quality model for Linked Data that specifies a set of quality characteristics and metrics related to those quality characteristics in the domain of Linked Data with a unique terminology as a reference for data quality specification and evaluation.

- Quality is an essential aspect in any product or service; and data quality is critical in many real-world use cases in both academia and industry because crucial decisions can be taken based on the data. Several standardisation bodies such as the International Organization for Standardization (ISO) and the World Wide Web Consortium (W3C) have recognised the importance of standard models for the specification of data quality. For instance, ISO produced the ISO 25012 data quality model, which is a generic quality model that does not include the particularities of Linked Data. In 2016, the W3C Data on the Web Best Practices Working Group produced the W3C Data Quality Vocabulary (DQV) as a unified ontology for describing the quality of data published on the Web in any format (CSV, XML, HTML, RDF, etc.). Nevertheless, existing quality characteristics and metrics for Linked Data found in the literature are not aligned with the models proposed by such standardisation initiatives. Reusing such data quality models and ontologies as the base and extending them with the particularities of Linked Data facilitate the better adaptation of the model in both academic and industrial communities. 
From a technological perspective, the following engineering problem must be solved:

- A quality model can guide the development of quality assessment tools by helping developers to identify quality metrics related to a given quality characteristic and to understand such metrics using their explicit semantics, which reduces the opportunity for ambiguities and misinterpretations. Furthermore, a quality model and its formal representation in RDF/OWL could improve the comparison of the quality assessment results generated by tools. Those models can be used for further tasks such as the selection of datasets with a sufficient level of quality or recommendation of Linked Data resources with particular characteristics. Nevertheless, there is a lack of Linked Data quality evaluation tools that are designed and implemented based on a quality model.

In order to achieve the second objective, from a methodological perspective, the following list of open research problems must be solved:

- As discussed in the introduction, data profiling is defined as the activity of creating small but informative summaries of data. Depending on their indented use, these summaries could include either simple statistics such as the number of instances of a given class or more complex relationships such as functional dependencies in data. Data profiling information can provide valuable inputs to quality assessment techniques. For instance, in relational data, which has a much mature research history of data quality, data profiling is an essential step in quality assessment [3]. Nevertheless, current Linked Data profiling techniques do not extract information with the level of detail required for quality assessment and repair. This hinders the usage of data profiling information in data quality assessment and repair techniques.

- Dataset descriptions play an important role in providing useful metadata about datasets. Depending on their intended use, a dataset description model can either capture fine-grained metadata with a lot of details or only the high-level coarse-grained metadata. In the state-of-the-art, there are (a) generic (i.e., suitable for any type of data, not only RDF) dataset description models such as DCAT ${ }^{1}$, and (b) RDF-oriented dataset description models such as VoID ${ }^{2}$, Dataset Description Vocabulary ${ }^{3}$, or Aether VoID extension ${ }^{4}$. Nevertheless, the majority of these dataset description models are focused on representing RDF

\footnotetext{
${ }^{1}$ https://www.w3.org/TR/vocab-dcat/

${ }^{2}$ https://Www.w3.org/TR/void/

${ }^{3}$ https ://www.w3.org/2001/sw/hcls/notes/hcls-dataset/

${ }^{4}$ http://ldf.fi/void-ext
} 
statistics; for instance, such models include LODStats [71] and RDFStats [144]. Analysing state of the art dataset description models it is evident that the current RDF dataset description models fail to represent fine-grained information about the vocabularies used and frequent patterns in datasets.

From a technological perspective, the following engineering problems must be solved:

- The availability of tools that can perform data profiling to extract fine-grained metrics from a dataset is one key requirement for quality assessment and repair methods based on RDF data profiling to be useful. Furthermore, it is important that these profiling outputs are represented using an explicit model that facilitates the reuse of such results in data quality use cases. Nevertheless, there is a lack of Linked Data profiling tools that are configurable in a declarative manner and that produce comprehensive profiling metrics with the level of detail required by data quality use cases.

- Currently the repositories that contain metadata about datasets in the Linked Open Data cloud only contain higher-level metadata or statistics about datasets. They do not contain granular fine-grained information that would be helpful for quality assessment and repair techniques. Thus, there is a lack of a repository of $\mathrm{RDF}$ data profiling information with the level of detail required for quality assessment tasks.

In order to achieve the third objective, from a methodological perspective, the following open research problem must be solved:

- For validating data quality requirements and performing the necessary actions for repairing data, a set of validation constraints or rules are necessary. Such validation constraints define the characteristics or quality standards that the data has to satisfy. In the state of the art methods, these validation constraints are mostly manually formulated by domain experts, which is a tedious process that requires a significant amount of manual labour. Furthermore, it requires human experts with good knowledge about the domain. Nevertheless, a vast amount of information about validation constraints is already implicitly embedded in the data and could be extracted by analysing the data themselves. However, there is a lack of methodologies for inducing integrity constraints from data and generating validation inputs, such as RDF Shapes. 
From a technological perspective, the following engineering problem must be solved:

- Automatic induction of integrity constraints using data profiling information and their representation using RDF Shapes will provide the foundation for many automatic quality assessment and repair techniques in the Linked Data domain. Nevertheless, there is a lack of tools that can induce RDF Shapes from data profiling information.

In order to achieve the fourth objective, from a methodological perspective, the following list of open research problems must be solved:

- The current Linked Data quality assessment techniques require a large amount of manual effort, which is not feasible for large-scale Linked Data datasets with billions of triples. There are efforts to address this challenge using crowd-sourced approaches [5, 141, 224]; however, this still requires much time and requires people with domain knowledge which could be costly. One of the challenging tasks in data validation is creating validation constraints with less manual effort, but currently there is a lack of automatic techniques for generating validation constraints following data-driven approaches.

- Most of the research on Linked Data quality is focused on the quality assessment aspect. This is natural as Linked Data quality research is still in an early stage and quality assessment is the first step towards ensuring data quality. Such assessments allow detection of low-quality portions of data. In some cases, this low-quality data can be repaired to improve the overall quality of a dataset. Nevertheless, there is a lack of repair techniques for data quality issues in Linked Data.

From a technological perspective, the following engineering problem must be solved:

- Most of the current state of the art quality assessment tools depend on manual human effort in different stages and thus, makes them unfeasible to be used with large scale datasets. There is a lack of automatic tools, frameworks, and data quality pipelines that support both quality assessment and repair based on data-driven approaches. 


\subsection{Contributions to the State of the Art}

The aim of this thesis is to provide solutions to the previously mentioned open research and engineering problems in order to fulfil the thesis objectives.

The first objective of this thesis is to to provide means for representing quality assessment results along with all associated metadata in a comparable manner, and this thesis has contributed to the advance of the current state of the art by providing:

\section{C1. A Linked Data Quality Model for representing Linked Data quality in a standard manner.}

This contribution is divided into one methodological contribution (C1.1) and one technological contribution (C1.2).

C1.1 A comprehensive quality model for Linked Data that provides a unique terminology and reference for Linked Data quality specification and evaluation. The quality model proposed in the thesis specifies a set of quality characteristics and quality measures related to Linked Data, together with formulas for the calculation of measures. It uses the generic ISO 25012 data quality model as its base and it is implemented as an extension of the W3C Data Quality Vocabulary that can be used to represent quality information specific to Linked Data. Data quality evaluation results based on the quality model are represented in RDF and can also be easily published as Linked Data. The quality model consists of 15 quality characteristics, 89 base measures, 23 derived measures, and 124 quality indicators.

C1.2 LD Sniffer - a software tool based on the quality model for validating the accessibility of Linked Data. Accessibility, which is defined as the degree to which the data can be accessed, is a highly relevant quality characteristic to achieve the benefits of Linked Data. LD Sniffer provides both a web-based interface and a command-line interface and it is developed as an open-source tool for performing quality assessments on the accessibility of Linked Data. It generates unambiguous and comparable assessment results with explicit semantics by using the definitions of both quality metrics as well as assessment results from the previously mentioned Linked Data quality model (C1.1).

The second objective of this thesis is to identify information that could be automatically generated on large-scale datasets and could be used for 
quality assessment and repair tasks. To this extent, this thesis presents new advances by providing:

C2. Loupe - A framework for Linked Data profiling is a set of methodological and technological contributions that provide models and tools for improving RDF profiling with the objective of making them more suitable for Linked Data quality assessment and repair use cases.

C2.1 The Loupe Extended Dataset Description Model is an extended dataset description model that is focused on representing fine-grained RDF data profiling metrics. It extends the information in existing RDF dataset description models, such as VoID [132] and LODStats [70], and proposes a comprehensive set of new metrics focused on data quality that capture further details such as vocabulary usage and triple patterns (e.g., which classes and properties are used in the dataset, and implicit domains, ranges, cardinalities of properties as seen in data), classes with common instances, frequent abstract triple patterns, or frequent subgraphs among others. The proposed extended dataset description model contains 84 Linked Data profiling metrics that are useful for quality assessment and repair tasks. Out of which, 33 metrics are completely novel and are not covered by any of the existing models.

C2.2 Loupe Linked Data Profiling Tools is a novel suite of tools that facilitate the automatic extraction of data profiling information from datasets using a set of parameterised SPARQL query templates and generating dataset descriptions according to the Loupe Linked Data profiling model (C2.1). The datasets can be either accessible as RDF data dumps or as public SPARQL endpoints. The Loupe framework also consists of (a) the Loupe web portal ${ }^{5}$ that allows the exploitation of this information in a user-friendly manner with visualisation support, (b) the Loupe API that is a RESTful service that exposes Loupe functionalities as a service, (d) the Loupe Linked Data profiling repository that contains the profiled data from a large number of publicly available datasets. We demonstrated the scalability of the framework by profiling 26 thousand datasets containing 34 billions of triples. The profiling results of all those datasets were added to the profiling repository. The Loupe API was integrated into other research projects such as MappingPedia [192]. The Loupe Web portal has been visited more than 40,000 times by $3000+$ unique visitors from 87 countries.

\footnotetext{
${ }^{5}$ http://loupe. linkeddata.es
} 
The third objective of this thesis is to propose methods for automatically deriving Linked Data validation constraints for large datasets. To this extent, this thesis presents new advances by providing:

C3. An automatic RDF shape induction method that follows a data-driven approach for inducing integrity constraints for RDF data using data profiling. The induced constraints are combined to form RDF Shapes that can be used to validate RDF graphs. The proposed method is based on machine learning for classification and regression, such as Random Forrest, Support Vector Machine (SVM), k-nearest neighbors (KNN), or Naive Bayes to automatically generate RDF shapes using profiled RDF data as features. We demonstrate that the proposed method could generate useful RDF Shapes and achieved 98.81\% F1 in deriving maximum cardinality constraints, $97.30 \% \mathrm{~F} 1$ in deriving minimum cardinality constraints, and $95.94 \%$ in deriving range constraints for a subset of English DBpedia data.

The fourth objective of this thesis is to develop methods for automatic quality assessment and repair. To this extent, we present new advances by providing:

C4. Methods for automatic quality assessment and repair using Linked Data profiling metrics and RDF Shapes.

C4.1 A method to predict inconsistent mappings based on Linked Data profiling metrics. This method is related to the consistency quality characteristic of the Linked Data Quality Model (C1). Many RDFbased Linked Data publishers use mappings in the RDF generation process to transform non-RDF source data into RDF. Nevertheless, as discussed in several studies $[62,63,220]$ in literature, not all mappings are correct and consistent. Because large-scale datasets, such as DBpedia, have a significantly large number of mappings to generate data, it is not feasible to inspect all such mappings manually to ensure their consistency. Thus, in this thesis, we propose a data-driven method to detect inconsistent mappings automatically by analysing profiling information from instance data extracted using the Linked Data profiling framework (C2). Such information is provided as input features to a classifier model built using machine learning that can identify inconsistent mappings automatically once it is trained. In this thesis, we demonstrated that the proposed method could achieve 93\% accuracy in identifying inconsistent mappings. These results helped to detect incorrect mappings in DBpedia and correct them. Furthermore, this approach inspired the idea for a Google Summer of Code project $^{6}$ in 2018, which the author co-mentored.

\footnotetext{
${ }^{6}$ https://github.com/dbpedia/GSoC/issues/15
} 
C4.2 A noise detection method for information extraction tools based on RDF Shapes. The noise detection approach proposed in the thesis is focused on detecting and removing noisy triples that are generated using automatic information extraction processes. In this context, we refer to any low-quality or less useful triples as "noisy triples". More concretely, we focus on three specific types of noisy triples, namely, Inconsistent, Overly Generic, and Factually False triples. Thus, this method is related to the consistency and accuracy characteristic of the Linked Data Quality Model $(C 1)$. The noise detection method proposes to first perform a classification of noise found in automatically-constructed datasets and then to use the Linked Data Profiling Framework (C2) and RDF Shapes (C3) to first filter out inconsistent triples before detecting overly generic and factually false triples. In this thesis, we demonstrate that the inconsistent triple detection, generic triple detection, and factual false triple detection could achieve precisions of $86.84 \%, 98.25 \%, 85.12 \%$ respectively.

C4.3 A link repair method based on RDF Shapes. The first and the fourth Linked Data principles are "Use URIs as names for things" and "Include links to other URIs, so that they can discover more things". Thus, it is essential to use global dereferenceable identifiers in the form of IRIs denoting entities. Nevertheless, it is a common problem that many entities that should be represented IRIs are being represented using literal strings [171]. Because of this some entities are not linked diminishing the advantages of using Linked Data. Thus, in this thesis, we propose an approach for identifying strings that denote entities and replacing them with their corresponding entity IRIs using RDF Shapes and data profiling information. Because this method makes the graph more consistent and increases the number of links, it is related to the completeness and consistency quality characteristics of the Linked Data Quality Model ( $C 1)$. The RDF Shape Induction technique (C3) makes it possible to identify property ranges, i.e., objects of a given property should be an IRI or a literal using data profiling metrics. Such information can be used to identify entities that are represented as strings. We demonstrate that the proposed method can achieve $98 \%$ recall and $76 \%$ precision in identifying such strings and $97 \%$ precision in converting them to their corresponding IRIs. Furthermore, the link repair method could add $25 \%$ more new links and improve the overall connectivity by $17 \%$.

C4.4 A type completion method using Linked Data profiling metrics. The type information about resources in Linked Data plays a vital role in making them queryable and reusable. Nevertheless, in some datasets there are a significant amount of resources without type information (DBpedia, 
1.1 million instances without a specific-type). This could be due to various reasons such as deficiencies in the data generation or transformation process (e.g., lack of mappings) or deficiencies in source data (e.g., missing type information). The fact that data is incomplete and could contain noise makes it difficult to use classic type inference by reasoning to complete the missing type information [187]. Thus, this thesis proposes a data-driven approach for predicting type information of instances utilising machine learning algorithms for classification and data profiling metrics defined in Linked Data Profiling Framework (C2) as input features. Because the goal of the proposed method is to complete the RDF graph by adding the missing type information, this method is related to the completeness quality characteristic of the Linked Data Quality Model (C1). We demonstrate that the proposed method can achieve an accuracy of $99.28 \%$ could be achieved when a dataset has only two classes and $95.63 \%$ when a dataset has 20 classes.

\subsection{Restrictions, Assumptions, and Hypotheses}

This thesis has been developed under a set of restrictions and assumptions that defines the scope of the thesis and help explain the decisions taken for the achievement of the thesis goals.

The following set of restrictions defines the limits of the contributions of this thesis and allows determining future research objectives. These restrictions delimit the research problem and allow the incremental improvement of research.

R1. The work presented in this thesis is only focused on Linked Data represented in using the RDF model [52]. We also limit our scope to two ontology languages; RDF Schema (RDFS) [43] and Web Ontology Language (OWL) [109]. In the future, the proposed methods can be expanded to non-RDF based knowledge graphs.

R2. The scope of the Linked Data Profiling Framework is limited to schema-based Linked Data profiling as that provides the most relevant information for Linked Data quality assessment and repair.

R3. The automatic RDF Shape Induction method is defined in a generic manner. Nevertheless, we currently do not cover all possible constraints in RDF Shapes. In this thesis, we focus on three types of constraints, namely, maximum cardinality, minimum cardinality constraints, and range constraints. The method can be extended to cover other types of constraints. 
R4. Quality assessment and repair techniques do not cover all quality dimensions. A subset of relevant quality dimensions was selected based on the use cases considered in this work.

R5. The proposed noise detection approach for information extraction tools (4.2) does not cover all types of noisy triples (low-quality or less useful triples). We limit our scope to three types of noise, namely, Inconsistent, Overly Generic, and Factually False triples.

R6. The proposed type completion method using Linked Data profiling metrics use $R D F S$ or $O W L$ classes (see R1) from a given ontology that are two-levels in the class hierarchy the owl:Thing as the candidates for type prediction. This avoids too-specific types with lesser number of instances to train the supervised method.

Similarly, the following set of assumptions were made regarding the work performed in this thesis.

A1 Existing dataset catalogs contain a representative sample of the Linked Open Data cloud. We have used LOD Laundromat [24] as the catalogs for extracting datasets for our experiments. It is a platform that harmonises and republishes Linked Data documents that are either crawled by them or submitted by the data publishers. Currently, it serves more than 37 billion triples from over 650,000 Linked Data resources. Given its scale and its objective, it is reasonable to assume that it contains a representative sample of the Linked Open Data cloud.

A2. SHACL is the suitable language for expressing RDF Shapes induced in this work. There are several languages available for expressing validation constraints for RDF such as SHACL [137], ShEX [194], and SPIN [138]. However, at the time, only SHACL is published as a W3C Recommendation with a through approval process from a well-established standardisation organisation. Thus, we have selected SHACL as the most suitable language for representing RDF Shapes generated in this work.

Once the assumptions have been identified and presented, the set of hypotheses of this thesis are described:

The first contribution of the thesis (C1), Linked Data Quality Model (LDQM), represents the diverse quality metrics for Linked Data found in the literature. Following hypotheses were tested related to $C 1$ : 
H1. The proposed Linked Data Quality Model (C1.1) satisfies a set of characteristics relevant a quality model, namely, comprehensiveness, applicability, understandability, and consistency.

H2. The proposed Linked Data Quality Model (LDQM) is useful for the development of Linked Data quality assessment tools (C1.2).

The second contribution of the thesis (C2), Loupe Linked Data profiling framework, consists of an extended dataset description model and a suite of Linked Data profiling tools. Following hypotheses were tested related to C2:

H3. The proposed Loupe dataset description model (C2.1) is more expressive than the current dataset description models and contains fine-grained Linked Data profiling metrics needed for quality assessment techniques and repair use cases.

H4. The Loupe Linked Data profiling tools (C2.2) are scalable, i.e., the execution time of the Loupe Linked Data tools scale with respect to the dataset size (in triples) and richness of vocabularies used (number of distinct classes and properties) and it can be used to profile large datasets (more than million triples) in the Linked Open Data cloud in less than 30 minutes.

The third contribution of the thesis (C3), an automatic RDF shape induction method, is a data-driven approach for inducing integrity constraints and RDF Shapes using machine learning algorithms with data profiling metrics from $C 2$ as features. Following hypotheses were tested related to $C 3$ :

H5. The proposed automatic RDF Shape Induction method (C3) has a higher accuracy than a baseline approach based on the most frequent value, i.e., a ZeroRbased algorithm [127] for generating RDF Shapes with maximum cardinality, minimum cardinality constraints, and range constraints (the scope is defined by $R 3$ ).

The fourth contribution of the thesis (C4), is a set of four methods based on Linked Data profiling metrics from C2 and RDF Shapes from C3. We have defined hypotheses for each of the methods. Following hypotheses were tested related to $C_{4}$ :

H6. The proposed method to predict inconsistent mappings based on Linked Data profiling metrics $(C 4.1)$ can achieve an accuracy of $80 \%$ in identifying inconsistent mappings. 
H7. The proposed noise detection approach for information extraction tools (C4.2) can achieve $80 \% \mathrm{~F} 1$ in detecting Inconsistent, Overly Generic, and Factually False triples (the scope is defined by $R 5$ ).

H8. The proposed link repair method based on RDF Shapes (C4.3) can achieve more than $80 \% \mathrm{~F} 1$ in identifying and repairing broken links in Linked Data (i.e., entities that should be denoted by IRIs but are represented as strings).

H9. The proposed type completion method (C4.4) using Linked Data profiling metrics can achieve an accuracy of $80 \%$ when predicting types for instances (the scope is defined by $R 6$ ).

\subsection{Evaluation Plan}

With respect to the hypotheses defined in this thesis, in order to accept or reject them, the following evaluations have been performed:

E1 (for H1) The goal of this evaluation is to verify that the proposed quality model satisfies a set of characteristics relevant a quality model, namely, comprehensiveness, applicability, understandability, and consistency. To this end, we analyse the quality model with respect to each of the characteristics. For instance, to validate the comprehensiveness, the publications of meta-studies such as literature surveys of the current quality characteristics and metrics as well as primary studies on specific quality metrics published in the literature are used to compile a representative list of quality characteristics and metrics for Linked Data. The information gathered this way are used to verify if the proposed model is comprehensive.

E2 (for H2) The goal of this evaluation is to validate that the proposed model is useful for the development of Linked Data quality assessment tools. To this end, we develop a quality assessment tool for evaluating the accessibility of Linked Data and analyse how the Linked Data quality model can help in each of the steps in the tool development process.

E3 (for H3) The goal of this evaluation is to validate the hypothesis that the Loupe dataset description model is more expressive than the current dataset description models in the state-of-the-art. To this end, the first task is to collect a set of requirements, in terms of expressivity, required by quality assessment tasks. This can be done by analysing the information needed for current quality assessment tasks in the literature as well as for the quality assessment tools seen in practice. Finally, this list of requirements is revised by a set of researchers in 
the field to ensure that it is representative and it is extended with any missing requirements. Once the expressivity requirements are finalised, the current dataset description models and the Loupe extended dataset description model are analysed to check their expressivity.

E4 (for H4) The goal of this evaluation is to validate that the execution time of Loupe tools scales linearly with respect to the dataset size (in triples) and richness of vocabularies used (number of distinct classes and properties). This can be done by performing an experiment in which a representative sample of LOD datasets are profiled and the execution time for each of those runs are recorded. For each dataset, dataset size and complexity are also extracted. Then, the recorded execution times are analyzed against those attributes to validate if the executions scale linearly.

E5 (for H5) The goal of this evaluation is to validate the hypothesis that the proposed automatic RDF Shape Induction method (C3) has a higher accuracy than a baseline approach based on the most frequent value for generating RDF Shapes with maximum cardinality, minimum cardinality constraints, and range constraints. To this end, we create a gold standard with manual annotations for each of the constraints that were considered in the RDF Shape induction method. Then the accuracy of the method is calculated using the gold standard data.

E6 (for H6) The goal of this evaluation is to validate the hypothesis that the proposed method to predict inconsistent mappings based on Linked Data profiling metrics (C4.1) can achieve an accuracy of $80 \%$ in identifying inconsistent mappings. To this end, a gold standard with a set of manually annotated with correct and erroneous mappings is created for evaluation purposes. In this work, DBpedia mappings from different localised DBpedia chapters such as English DBpedia, Spanish DBpedia, German DBpedia, Dutch DBpedia, and Greek DBpedia are used for annotating the mappings. Then, the accuracy of the proposed technique for detecting erroneous mappings is measured. Then these metrics are used for validating the hypothesis.

E7 (for H7) The goal of this evaluation is to validate the hypothesis that the proposed noise detection approach for information extraction tools (C4.2) can achieve 80\% F1 in detecting Inconsistent, Overly Generic, and Factually False triples. To this end, a gold standard with a set of manually annotated with Inconsistent, Overly Generic, and Factually False triples is created for evaluation purposes. Then, the precision and recall of the proposed method is measured calculated and used to validate the hypothesis. 
E8 (for H8) The goal of this evaluation is to validate the hypothesis that the proposed link repair method based on RDF Shapes (C4.3) can achieve more than $80 \% \mathrm{~F} 1$ in identifying and repairing broken links in Linked Data. For calculating the recall, a sample of data is manually annotated to identify all strings that should be represented as links using proper IRIs and use the recall of that sample to extrapolate the results of the techniques. For calculating the precision, a sample of the results of the technique is manually evaluated by a human reviewer.

E9 (for H9) The goal of this evaluation is to evaluate the hypothesis that the proposed type completion method $\left(C_{4} .4\right)$ using Linked Data profiling metrics can achieve an accuracy of $80 \%$ when predicting types for instances. For this method, we have created a gold standard with the following steps. For English and Spanish DBpedia datasets, we select a sample of data from each dataset. For the selected sample data, we remove the type information from each instance and used the prediction models to predict the type only using the Linked Data profiling metrics. Then, we compare the predicted type information with the original dataset (the gold standard) for testing the performance of the proposed method.

\subsection{Research Methodology}

This section presents an overview of the research methodology followed during the development of this thesis. The research methodology outlines the methodological and technical inputs that were used to achieve the objectives of this thesis (Section 3.1) and to provide several contributions to open research problems, as described in Section 3.3. These contributions are then combined together in order to achieve each specified goal. Furthermore, this section provides details of the followed research process (Section 3.6.2). Figure 3.1 shows an overview of the thesis with the use cases that motivated the objectives of the thesis, its main contributions, and the applications.

\subsubsection{Methodological and Technological Inputs}

In order to deliver the contributions of this thesis, methodological and technological inputs are taken into account as presented.

In order to deliver contributions related to the objective of providing means for representing quality assessment results along with all associated metadata in a comparable manner using well-established standards, the following inputs were taken into account: 


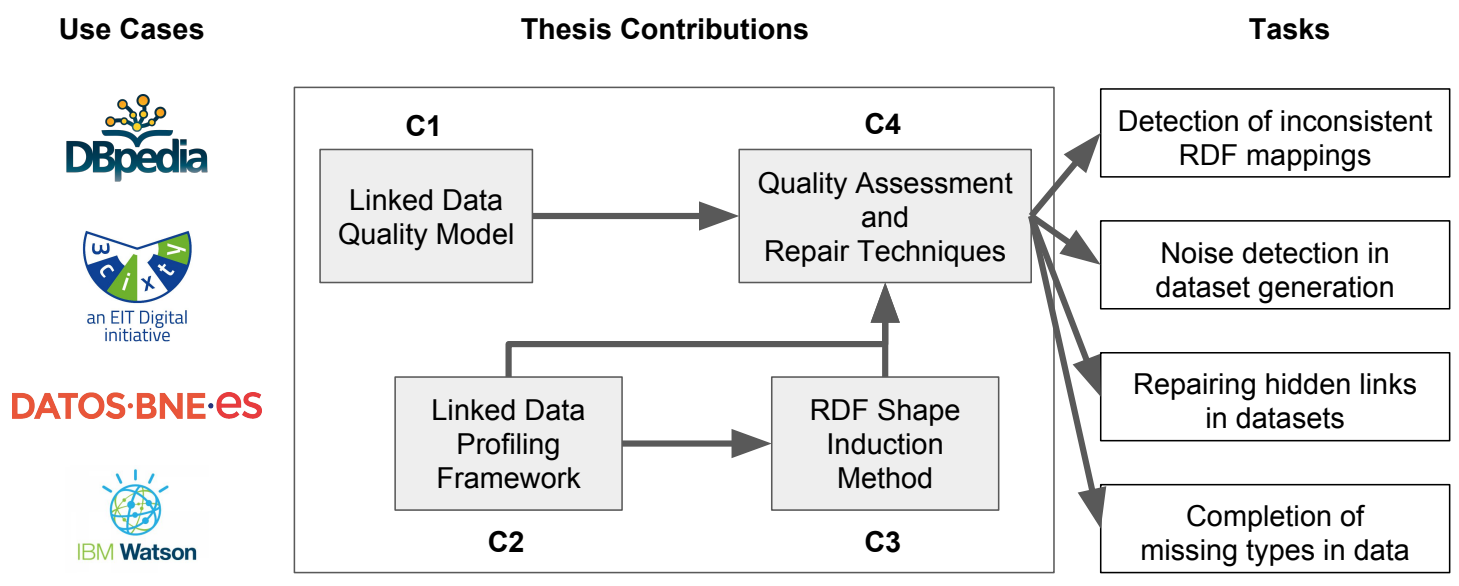

Figure 3.1: Thesis overview

1. Methodological guidelines for performing literature reviews. Part of the methodology followed in the thesis relies on the literature reviews performed in the fields of interest. For performing such literature reviews, methodological guidelines by Kitchenham [135] have been followed.

2. Existing Linked Data quality assessment tools. The existing tools related to Linked Data quality assessment and repair were analysed in order to obtain an overview of the requirements of representing quality assessment requirements and results in Linked Data.

3. State of the art analysis of Linked Data quality metrics, characteristics, and quality models. This analysis, presented in Section 2, together with the previous input helped in identifying the current diverse quality metrics and terminology used in the literature.

4. Data quality standards. Because Data quality is a crucial aspect for many industries, several data quality standards are developed by standardisation bodies such as International Organization for Standardization (ISO) and World Wide Web Consortium (WWW). These existing data quality standards are used as input for creating a comprehensive Linked Data quality model.

In order to provide contributions related to the objective of identifying infor- 
mation that could be automatically generated on large-scale datasets and could be used for quality assessment and repair tasks, the following inputs were taken into account:

1. Existing RDF data profiling techniques. Existing RDF data profiling techniques presented in Section 2, were analysed in order to understand the capabilities and limitations of the current RDF profiling methods in the context of quality assessment.

2. Existing RDF dataset description models. Currently, the RDF dataset profiling results are represented using RDF dataset description models so that they can be utilised by the RDF quality assessment and repair tools. Current RDF dataset description models were analyzed to understand their expressiveness and suitability in the context of using RDF data profiling information as input to the Linked Data quality assessment and repair tools.

In order to provide contributions related to the objective of proposing methods for automatically deriving Linked Data validation constraints for large datasets, the following inputs were taken into account:

1. Existing machine learning algorithms. Existing machine learning algorithms were studied to find the most suitable algorithms to be used for RDF Shape induction.

2. Manually annotated benchmarks Several manually constraints were created for using as training data for RDF Shape Induction techniques.

In order to provide contributions related to the objective of developing methods for automatic quality assessment and repair, the following inputs were taken into account:

1. Existing data quality assessment and repair techniques Existing data quality assessment and repair techniques were analysed to understand what are the capabilities of current techniques, their limitations, and to envision how those techniques can be improved with data profiling information.

2. Manually annotated benchmarks For each of the four methods, manually annotated benchmarks were created to be used as the gold standards for evaluating the method. 


\subsubsection{Description of the Research Process}

In the development of this thesis, an incremental process has been followed leading to four different phases: initialisation, development, implementation, and refinement.

During the initialisation phase, the state of the art was studied to understand the current status of the Linked Data quality assessment and repair techniques as well as to identify the current challenges and open research questions. In addition to the state of the art, a set of practical Linked Data use cases including the Spanish DBpedia project, the Spanish National Library (BNE) project, and the 3Cixty project were studied to identify the relevant quality issues that need to be resolved. With this information, the objectives of the thesis, research questions, and the hypotheses were defined. For each of the objectives of the thesis, development and evaluation phases were carried out in an iterative manner.

In order to achieve the first objective:

- During the development phase, the quality model was defined using a bottom-up approach by identifying the quality measures and indicators in the state of the art and by classifying them to base measures, derived measures and indicators. Furthermore, relationships among different measures were identified. Then, a conceptual model for Linked Data quality is created and it is implemented as an ontological representation.

- During the evaluation phase, the quality model was evaluated for a set of criteria including comprehensiveness, applicability, understandability, and consistency. Furthermore, the usefulness of the quality model was evaluated by a practical use case of building a quality assessment tool guided by the quality model.

In order to achieve the second objective:

- Similar to the previous objective, a bottom-up approach was used for developing the Linked Data profiling framework. The use cases related to Linked Data profiling were collected and the tasks related to Linked Data profiling were identified.

- During the evaluation phase, the Linked Data profiling framework was evaluated for two aspects: (a) expressivity of the profiling framework to capture the finegrained information and (b) the scalability of the execution times with respect to the dataset size and the richness of vocabularies used.

In order to achieve the third objective:

- During the development phase, a generic shape induction method is developed by formulating each constraint generation problem as a machine learning problem. 
Profiling information is used as the inputs to these machine learning problems. A set of training data is created by manually annotating the data. A set of machine machine learning algorithms are tested for their accuracy for the given problem using the manually annotated data and a set of models are created for generating RDF Shapes.

- During the evaluation phase, data from DBpedia, 3Cixty, and the Spanish National Library (BNE) were manually annotated to create gold standards for evaluating the RDF Shapes generated by the RDF Shape Induction method.

In order to achieve the fourth objective:

- During the development phase, a set of specific use cases were identified for quality assessment and repair. Then, for each use case, a set of relevant profiling information and RDF shapes are chosen to develop quality assessment and repair methods.

- During the evaluation phase, a set of manually created gold standards were used to measure the correctness of the proposed approaches.

Finally, during the refinement phase, the inputs from the evaluations were used to improve the contributions related to each of the four objectives. 


\section{Chapter 4}

\section{Linked Data Quality Model}

As discussed in Chapter 3, the first objective of the thesis is to propose a quality model for Linked Data quality specification, adhering to the well-established ISO standards for data quality and to the W3C Data Quality Vocabulary so that the quality assessment results about distinct datasets can be published in a standard and interoperable manner. In this chapter, we present Linked Data Quality Model proposed in this thesis.

Various initiatives exist with the common goal of specifying the quality of Linked Data and evaluating Linked Data datasets. Quality assessment in these initiatives, however, is quite diverse since different authors focus on different aspects of Linked Data, on different characteristics (e.g., completeness, licensing, accuracy), and on different measures for these characteristics (e.g., missing links, indication of attribution, semantically incorrect values). Furthermore, some authors have developed methodologies and tools for Linked Data evaluation, which are also characterised with a high diversity in terms of the evaluated characteristics and measures.

Quality models are important for providing consistent terminology and guidance for quality assessment and are the basis for the evaluation of any product or service. This is especially significant for the integration of evaluation results and benchmarking, which is one important aspect of evaluation [91, 136], and without a quality model it is sometimes difficult to integrate evaluation results, perform benchmarking, or to select products or services according to their quality. Because of this, the ISO recognised the need for a quality model for data, and produced the ISO 25012 quality model [114]. However, the ISO data quality model can be regarded as very general and, furthermore, it does not include particularities of Linked Data. The W3C has also recognised the need for having a unified ontology for describing data quality and produced the W3C Data Quality Vocabulary [10] (DQV) ${ }^{1}$ within the W3C Data on the Web Best Practices Working Group. Nevertheless, DQV aims to be a lightweight

\footnotetext{
${ }^{1}$ http://www.w3.org/TR/vocab-dqv/
} 
ontology suitable for any type of data on the web (e.g., CSV, XML, HTML, RDF, etc.), thus it is generic and does not address the specific characteristics of Linked Data. Furthermore, it only provides a base framework for describing quality metrics and measures but does not define concrete quality metrics, which are expected to appear in quality models. Nevertheless, a data quality model could use DQV as the base ontology for representing the quality model elements as Linked Data.

Motivated with the previous discussion, this chapter presents a quality model for the evaluation of Linked Data. It is a hierarchical quality model that provides unique terminology and that describes quality elements (i.e., a set of quality characteristics and a set of measures) related to Linked Data. The quality model presented in this chapter has been defined relying on the state of the art in Linked Data quality evaluation and specification and extends the ISO 25012 data quality model. Unlike the current state of the art, our quality model formalises a classification of different types of quality measures, and defines some measures, together with their related formulas that have not been specified in the literature.

This chapter also presents an extension of the W3C Data Quality Vocabulary in order to provide means to describe the particularities of Linked Data quality, together with the Linked Data representation of the quality model according to the mentioned extension (RDF instances), with dereferenceable URIs of all the quality model elements. By using these artefacts, it is possible to capture the evaluation results of any particular Linked Data dataset, as well as to make unique references to the evaluated metrics. This can ease interoperability and provide better integration of various evaluation efforts.

\subsection{Preliminaries}

\subsubsection{Quality Models}

A quality model is defined through a set of specific quality characteristics, quality sub-characteristics, quality measures, and through the relationships between these characteristics and measures $[19,114,115]$ and, to this extent, represents a specification of quality-related information. Quality measures defined in a quality model capture some information about quality characteristics and sub-characteristics and, usually, a classification of different types of quality measures is specified in the quality model $[113,196]$ :

- Base measures are measures that are a direct output of an evaluation; they can be related to one part of an evaluation (i.e., one test) or to the whole evaluation. An example of a base measure for web browsers can be startup time, memory consumption or number of open tabs in a single test. 
- Derived measures are measures obtained by combining different base measures. An example of a derived measure for web browsers can be memory consumption per open tab in a single test.

- Indicators are measures that are obtained by combining base and/or derived measures (e.g., from a number of tests), and are related to a whole evaluation. An example of an indicator for web browsers can be average startup time, or average memory consumption per tab.

Although the classification of the previously specified types of quality measures should be specified for each quality characteristic in a quality model, in the cases where a simple quality measure can sufficiently describe a sub-characteristic, derived and/or base measures are not necessary and quality indicators can be defined as a direct output of the evaluation. An example of such case can be an indicator that describes multilingual support of a web browser.

In some cases, additional inputs in the evaluation process are required in order to evaluate a specific measure (e.g., page loading time requires a set of specific web pages to load). Furthermore, these inputs are necessary in some cases in order to obtain enough information that enables the evaluation of a measure (e.g., for evaluating domain consistency in a triple of a dataset, it is necessary to obtain information about the ontology used for representing a dataset and the domain of a property found in the observed triple).

Where possible, for each quality measure, relationships should be defined formally (e.g., in terms of formulas) and each indicator is assigned as a measure of some quality sub-characteristic. For example, average startup time measures the browser time behaviour sub-characteristic, which can be defined as a sub-characteristic of the time behaviour characteristic.

Hierarchical structures of quality measures, as defined above, contribute in a better understanding of quality measures and their relationships [31]. Furthermore, especially having in mind information such as formulas, the evaluation of quality measures is more straightforward and an easier task when their complete hierarchy is defined.

Quality models are accepted as a valuable resource in quality assessment and specification and, in this context, they are used as a reference to the quality measures to be evaluated. By providing important details about quality measures, such as definitions, scales or formulas, quality models provide a guidance on which measures are important for evaluation and how to measure them. Various quality models have been described in the literature, both generic ones as domain-specific ones.

When it comes to data quality, the International Organization for Standardization (ISO) recognised the need for a data quality model and produced the ISO 25012 
(SQuaRE) data quality model [114]. According to such standard, data quality is "a common prerequisite to all information technology projects".

The ISO 25012 quality model defines fifteen data quality characteristics classified into inherent data quality characteristics and system-dependent data quality characteristics. The standard also recognises that the data quality characteristics can have different priorities in different cases. Furthermore, the standard allows that, depending on the use case, some characteristics can be excluded or that new ones can be added. This has been a common practice in software engineering, where various researchers have developed domain-specific software quality models based on the generic ISO 25010 software quality model [115] by introducing new quality characteristics and sub-characteristics. In order to extend an existing quality model, researchers usually use methods that are based on a top-down approach, such as the ones presented by Franch and Carvallo [83] or by Behkamal et al. [25], or methods that are based on a bottom-up approach, such as the one described by Radulovic et al. [196]. Furthermore, Dromey suggests that both approaches can be important for building quality models [65].

The top-down approach for extending quality models starts from an existing generic quality model, i.e., adopts an existing quality model and defines new quality characteristics and sub-characteristics. It then continues with the definition of quality measures for measuring these quality sub-characteristics and of the relationships between these measures. On the other hand, a bottom-up approach starts by defining a hierarchy of quality measures and the relationships between them. These relationships are typically defined in terms of the formulas used for the calculation of these measures. Once the quality measures are defined, a hierarchy of quality sub-characteristics and characteristics is constructed, which are then aligned to quality characteristics from an existing generic quality model.

In order to describe general quality model and evaluation result information that are in line with ISO standards, two ontologies have been provided in the state of the art. The Quality Model ontology ${ }^{2}$ (QMO) defines a generic ontology for representing quality models and their resources in any particular domain; it can be used as a generic ontology for specifying quality. The main classes of QMO are based on the ISO standards and it also uses the ISO terminology. Apart from the classes for describing quality measures (base measures, derived measures and indicators) and quality characteristics, QMO provides the means to describe units of measurement and measurement scales for each quality measure. Also, QMO provides a number of properties for describing relationships between the quality measures. In the context of the conceptual model, QMO provides means to describe the data related to the Linked Data quality model, since it is intended to be a general ontology.

\footnotetext{
${ }^{2}$ http://purl.org/net/QualityModel\#
} 
The Evaluation Result ontology ${ }^{3}$ (EVAL) defines a generic ontology for representing results obtained in an evaluation process; it is a ontology for representing the results of a quality assessment and is an extension of QMO. The classes of this ontology provide means for capturing the specific values obtained in an evaluation process and for relating such values to quality measures and evaluated subjects (e.g., a specific dataset). They also provide the possibility to describe measurement scales and units of measurement for the obtained values, as well as inputs in the evaluation. In the context of the conceptual model, EVAL provides means to describe quality values and evaluated datasets.

\subsubsection{Linked Data Quality Specification}

To the best of our knowledge, there is no clearly defined quality model for Linked Data. However, various efforts over the years have contributed to the understanding and quality specification of Linked Data. These efforts are mostly concentrated on quality evaluation of Linked Data datasets, as well as on the theoretical aspects of Linked Data quality.

Unlike the ISO standards, the literature describes the quality of Linked Data using different terminology. Characteristics of a dataset are called dimensions by Zaveri et al. [235], which are assessed with quality indicators. Furthermore, according to Bizer and Cyganiak [32], a procedure for measuring a data quality dimension is called indicator, measure, or metric.

Zaveri et al. [235] provide in their work a comprehensive review of the various efforts related to Linked Data quality specification and evaluation, with a comprehensive classification of quality dimensions and metrics found in the literature. It presents 69 metrics grouped into 18 quality dimensions extracted from 30 Linked Data quality related papers published from 2002 to 2014 . The conclusion that can be made from their work is that the efforts described are quite diverse in terms of the dimensions evaluated and the calculated measures. Since 2014, Behkamal et al. published a set of 10 metrics to assess 18 quality issues they identified [26]. Besides, Albertoni et al. proposed a metric for estimating the multilingual information gain through a linkset [11].

There are several tools in the literature that support quality assessment of Linked Data $[5,24,32,37,69,74,96,99,101,106,142,157,163,183,206]$. These tools vary in their scope for assessing quality. On the one hand, there are tools that are focused on assessing quality along one dimension, such as trust (TrustBot [99], Trellis [96], tSPARQL [106]) or interlinking (LinkedQA [101], LiQuate [206]). On the other hand, there are tools that are frameworks for generic quality assessment such as Luzzu [57], RDFUnit [142], or Sieve [157]. Furthermore, there is a set of tools that

\footnotetext{
${ }^{3}$ http://purl.org/net/EvaluationResult\#
} 
allows an exploratory inspection of quality issues such as ProLOD [37], LODStats [69], ABSTAT [183], and Loupe [163] that mainly use different statistics and patterns extracted from data. The quality model proposed in this chapter can be used by all these tools.

With respect to the state of the art described in this section, the quality model presented in this chapter contributes in several ways: i) it provides a unified reference for Linked Data quality, by describing a set of quality characteristics and measures, together with definitions and formulas; ii) it introduces a hierarchy of quality measures as described in the beginning of Section 4.1.1, relying on the current state of the art and extending it with the mentioned hierarchy in the case of each measure; and iii) it describes important details of each quality measure, such as quality aspects related to Linked Data, units of measurement or measurement scales.

\subsubsection{Existing Quality Specification and Assessment Ontolo- gies}

A number of ontologies, related to quality specification and assessment have been developed in the Semantic Web / Linked Data field to this date. These ontologies represent quality meta-models that can be used for describing quality-related information found in quality models. Next, we give a brief overview of the most relevant ontologies in the context of Linked Data quality.

The Data Quality Management Vocabulary (DQM) [87] is an ontology for representing data quality management activities in Semantic Web architectures. The main concepts of this ontology include data quality requirements, i.e., quality-relevant expectations on data and data quality reports with data quality scores. The goal of the DQM ontology is to automate the creation of quality reports based on the data quality requirements defined using the DQM ontology with a data quality score based on each requirement. Further, by using Semantic Web technologies it aims to do automated consistency checking between a set of data quality requirements and also to facilitate the exchange of both data quality requirements and data quality results. Unlike the conceptual model, DQM provides classes that are specifically related to some concrete aspects of quality (e.g., a class for denoting that a property is missing in a dataset).

The Dataset Quality Ontology (daQ) [56] is an ontology for representing the quality of a dataset. The ontology defines the classes related to quality category, dimension, and metric, and several properties to define the relationships between these classes. The classes and properties in daQ are defined as abstract and, therefore, they are not directly used. Instead, the intended use of this ontology implies the creation of specific classes and properties defined as subclasses and sub-properties of those defined in daQ. This means that, unlike in QMO and EVAL (Section 4.1.1), 
where the elements such as measures and characteristics are defined as instances, when using daQ these elements are mainly defined as classes. In the context of the conceptual model, daQ is equivalent to QMO, with the difference of the usage of different terminology.

The Data Quality Vocabulary ${ }^{4}$ (DQV) [10] is an ontology for representing the quality of datasets that has been developed by the W3C. Similarly as daQ, and unlike QMO and EVAL, DQV is an ontology specifically developed having in mind datasets. DQV provides classes and properties for capturing information about quality categories, dimensions and metrics of a dataset, as well as about quality certificates, standards and provenance related to a dataset. In the context of the conceptual model, DQV tends to provide the means for capturing both the details about quality (i.e., characteristics and measures) and about quality values (results of evaluation). Furthermore, although DQV is specifically designed for datasets, it does not provide the means to describe some specific aspects of Linked Data.

With respect to the state of the art, the proposed quality model contributes with the extension of existing ontologies in order to enable capturing information related to Linked Data which is not covered by the existing ontologies, as well as with bringing existing ontologies under unique umbrella by connecting their semantically related concepts.

\subsection{Quality Model for Linked Data}

This section describes a quality model for Linked Data and how it was defined using the bottom-up methodology proposed by Radulovic et al. [196]. The starting point for the definition of the quality model was the state of the art in Linked Data quality assessment and specification, and in particular the work done by Zaveri et al. [235]. Since the quality model presented in this section describes a classification of quality measures (i.e., base measures, derived measures and indicators), we have decided to adopt the terminology as described by the ISO standards.

The work by Zaveri et al. does not specify any base measures, derived measures nor indicators per se, and it does not specify a classification of quality measures, as the mentioned one that has been adopted in our work. Rather, Zaveri et al. define metrics which in different cases are related to different types of measures in our classification. In some cases, a metric described by Zaveri et al. appears in our quality model as a base measure, and in these cases we have used this measure in order to define derived measures and/or indicators that do not appear in the work by Zaveri et al. In other cases, a metric described by Zaveri et al. appears in our quality model as an indicator, and in these cases we have defined the base and/or derived measures

\footnotetext{
${ }^{4}$ http : //Www.w3.org/TR/vocab-dqv/
} 
that are used to calculate these indicators.

The main motivation for presented quality model and defined hierarchy of measures lies in: i) providing higher level of details about measures and their relationships, which are derived from the state of the art and the community has found them useful and has used them in practice; ii) aligning with the well-known quality model structure defined in the ISO standards.

Data quality is a multifaceted concept and different quality measures in a quality model can be related to different aspects of quality. The Linked Data quality model that we propose encompasses the different aspects of Linked Data quality, as illustrated in Figure 4.1, and all quality measures are classified according to these aspects.

The aspects of Linked Data quality can be categorised into two main groups: i) aspects related to inherent data quality; and ii) aspects related to the infrastructure that is used for serving the data. For instance, on the one hand, quality characteristics such as the accuracy of facts represented in Linked Data or the completeness of a dataset are intrinsic to the data themselves. On the other hand, quality characteristics such as response time of a Linked Data resource or support for different media types through content negotiation depend more on the capabilities of the server applications and hardware devices that are used to serve the data. The same dataset hosted in different infrastructures and system configurations could have different levels of quality.

The motivation for identifying the aspects associated with Linked Data and annotating the quality measures with the relevant aspect is to help quality evaluators to select the most relevant metrics depending on their quality requirements and to have a better understanding about them. For instance, Linked Data providers will know that any measures related to infrastructure will have to be re-evaluated if the provider changes the Linked Data servers and other publishing infrastructure.

The Domain data aspect refers to the concrete facts contained in the dataset. The quality of the domain data can be measured with respect to different quality characteristics such as accuracy, completeness, or timeliness. For instance, in a dataset about cities of Spain, a fact such as that the city of Madrid has a population of $3,165,235$ inhabitants could exist. This fact can be assessed to verify that it is correct and not outdated (i.e., it reflects the situation in the real world) so that it has sufficient quality for a given use case. Further, if the use case requires the dataset to have information about all the cities in Spain the completeness can be verified by checking the dataset against a list of all Spanish cities from an official dataset from the Spanish government.

The Metadata aspect refers to the information that provides the context and additional information about the domain data or conditions on the usage of data. The quality of metadata can be measured with respect to quality characteristics such as compliance or trustworthiness. For instance, for a dataset to be fit for a given 


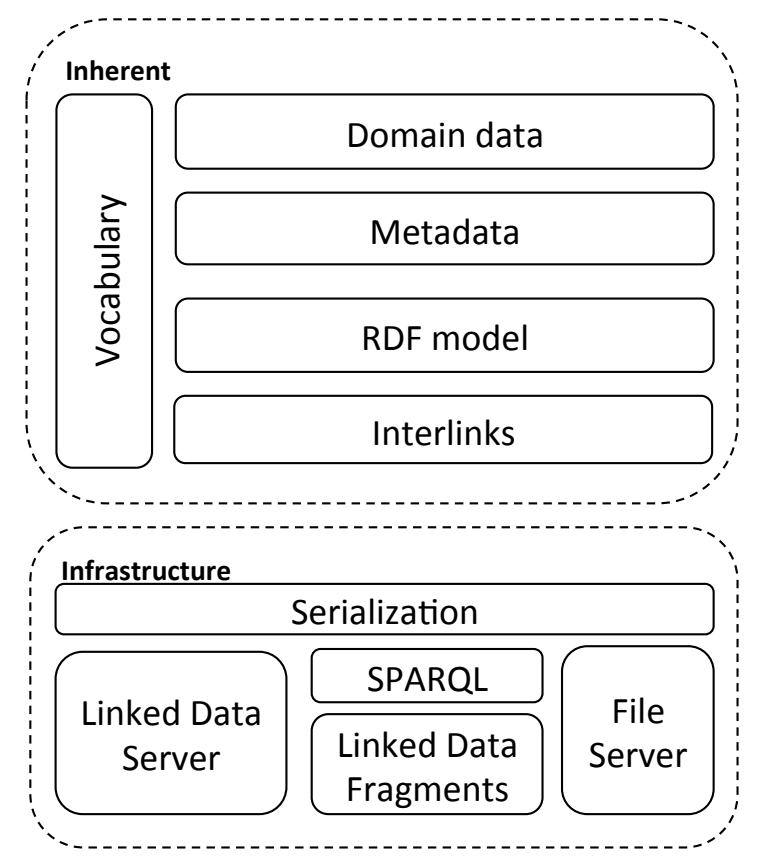

Figure 4.1: Aspects of Linked Data quality

use case, the data consumer may require to know about the provenance information, such as the provider of the data and its source, or the license information, so that a consumer can evaluate whether she can legally use the data for a concrete use case in commercial settings.

The Vocabulary aspect refers to the selection of vocabulary (ontology) terms representing both domain data and metadata. The ontologies used can be evaluated with respect to quality characteristics such as interoperability, conciseness, or understandability. For instance, if the common standard ontologies such as FOAF, DC Terms, SKOS, PROV that facilitate interoperability are used and if the ontologies used have dereferenceable identifiers with appropriate documentation that increase their understandability.

The RDF model aspect refers to different designs that are taken into account when modelling the domain data and metadata as RDF data. The quality of the RDF data model can be measured with respect to quality characteristics such as representational conciseness or the irregular use of RDF features such as collections, containers, or reification. For example, the use of collections without valid properties such as first and rest properties can violate the representational conciseness and affect performance.

The Interlinks aspect refers to exposing the RDF data as Linked Data and to linking the data to other relevant data so that consumers can discover more related 
data with the follow-your-nose approach. The quality of Linked Data interlinking can be measured with respect to quality characteristics such as accessibility or representational conciseness. For instance, in addition to proper RDF modelling, data can be made more useful by applying the Linked Data principles so that entities are named using HTTP URIs, useful information is provided when those URIs are looked up, and the entities are linked to the other related entities.

The Infrastructure aspects identify the approaches that are commonly used to expose Linked Data, such as Linked Data servers, SPARQL and Linked Data Fragments endpoints, or RDF dumps in file servers. The quality of the infrastructure can be measured with respect to quality characteristics such as availability, performance, or compliance. Linked Data servers such as Pubby or Elda are used to expose Linked Data as dereferenceable Linked Data resources via the HTTP protocol and they are associated with properties such as response time, throughput, or the different media types supported.

The Serialization aspect refers to the representation of RDF data in some RDF serialization format such as Turtle, RDF/XML, JSON-LD, N3, N triples, N quads, or Trig. In the case in which Linked Data are available as bulk download, the serialization could be a compressed archive such as a zip or a tarball archive. The quality of the representation can be measured with respect to quality characteristics such as syntactic accuracy, interoperability, or versatility. For instance, if an RDF document is serialised using RDF/XML the serialised representation of the model should follow all the syntactic rules defined by the RDF/XML Syntax specification [23].

Apart from this categorization in terms of aspects, Linked Data quality encompasses different levels of RDF concepts, including: i) IRIs/Blank nodes/Literals; ii) individual statements (i.e., triples); iii) RDF graphs; and iv) RDF datasets as a whole. These different levels are shown in Figure 4.2, and each quality measure in the Linked Data quality model is related to one of these levels.

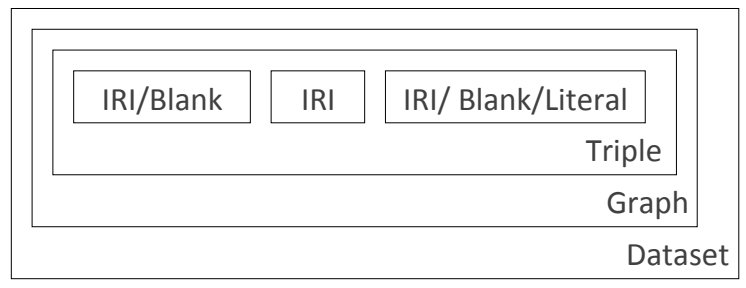

Figure 4.2: Data model (RDF) levels

The following sections present how the bottom-up approach has been used in order to define the Linked Data quality model. Due to space reasons we cannot present the quality model definition in complete details; therefore, for illustration purposes 
we present the outcomes of each step in the bottom-up methodology related to only a subset of the quality model. For each quality measure presented, we emphasise the aspect it measures (Figure 4.1) and the level on which the measure is calculated (Figure 4.2).

The complete overview of our quality model can be found at the Linked Data quality model wiki $^{5}$.

\subsubsection{Identification of Base Measures}

The first step in building the Linked Data quality model was to identify the base measures. In total, we have defined 89 base measures, out of which 44 directly come from the survey by Zaveri et al. [235], and 45 have been newly introduced in our work. These measures are described in detail in the quality model wiki and are classified according to the quality characteristic that they are related to.

With respect to the RDF data model levels (Figure 4.2), each base measure can be related to any of such levels. For example, base measures related to IRIs include:

- IRI dereferenceability. Whether an IRI is derefenceable or not. This measure is related to the Infrastructure aspect (Figure 4.1), and possible values for this measure are true and false.

- Short IRI. Whether an IRI is short or not. This measure is related to the RDF model aspect, and possible values for this measure are true and false.

In some cases, base measures can be related to a triple in a graph or a dataset. An example of such a base measure includes:

- Subject dereferenceability. Whether a subject in a triple is dereferenceable or not. This measure is related to the Infrastructure aspect, and possible values for this measure are true and false.

Depending on the context of their use in the evaluation, i.e., in the calculation of derived measures or indicators, some base measures can be related both to the IRI or triple level. An example of such a measure is:

- Subject types. A list of classes that an instance represented by an IRI (or a subject in a triple) is type of. This measure is related to the Vocabulary aspect, and possible values for this measure are any ontology class.

The previous base measures are related to the IRIs or triples in a dataset, and these measures alone can be useful in the process of error correction and data repair. Finally, an example of base measures that are related to a graph or a dataset include:

\footnotetext{
${ }^{5}$ http://delicias.dia.fi.upm.es/LDQM
} 
- Number of interlinked subjects. The total number of all subjects in a dataset that are linked.

- Number of subjects. The total number of subjects in a dataset.

- Number of IRIs. The total number of IRIs in a dataset.

- Number of triples. The total number of triples in a dataset.

Number of interlinked subjects base measure is related to the Interlinks aspect and the rest of the base measures are related to the Domain data aspect and the value for each of these measures can be any natural number.

\section{Identification of derived measures}

In this step, the previously defined base measures are used in combination with the inputs in the evaluation (e.g., an ontology) in order to define derived measures. Similarly as in the case of base measures, derived measures for Linked Data datasets can be related to different RDF data model levels. In total, 23 different derived measures have been defined, which are described in detail in the quality model wiki, and are classified according to the quality characteristic that they are related to. In total, 6 derived measures come directly from the survey by Zaveri et al., and 17 derived measures have been newly introduced in our work.

By analysing all the defined base measures, we identified several patterns of defining derived measures that will be used by the quality model presented in this chapter.

Some patterns are related to the aggregation of base measures in a higher RDF data model level. When a base measure of a lower level such as an IRI is measured, it can be aggregated to come up with derived measures that are associated with a higher level such as triples, RDF graphs or datasets. An example of such aggregation is:

- Number of dereferenceable IRIs. The total number of dereferenceable IRIs. This measure is related to the triple, graph or dataset levels, and is defined using IRI dereferenceability, which is an IRI level base measure. Furthermore, this derived measure is related to the Infrastructure aspect, and possible values for this measure are any natural number.

- Number of dereferenceable subjects. The total number of dereferenceable subjects. This measure is related to the graph or dataset levels, and is defined using Subject dereferenceability, which is an IRI level base measure. Similarly as in the previous case, this derived measure is related to the Infrastructure aspect, and possible values for this measure are any natural number. 
Other patterns are related to the interpretation or the combination of base measures that are related to the same RDF data model level. Sometimes, it is possible to combine or interpret various base measures in order to define new derived measures that are on the same RDF data model level as the base measures used for their definition. Examples of such derived measures include:

- Disjoint classes. Whether an instance represented with a specific IRI is an instance of disjoint classes. This measure is related to the IRI level and is defined using Subject types, an IRI-level base measure. Furthermore, this derived measure is related to the RDF model and Vocabulary aspects, and possible values for this measure are true and false. In order to calculate this derived measure, an ontology is needed as an input in the evaluation in order to examine ontology classes and axioms and to obtain information about disjoint classes to be compared with types of the observed instance in a dataset.

- Domain consistency. Whether the type of a subject in a specific triple is consistent with the domain of a property of a triple. This measure is related to the triple level, and is defined using Subject types, a triple-level base measure. Furthermore, this derived measure is related to the RDF model and Vocabulary aspects, and possible values for this measure are true and false. In order to calculate this derived measure, an ontology is needed as an input in the evaluation in order to examine properties and to obtain information about property domain to be compared with the type of the observed triple in a dataset.

\subsubsection{Identification of Indicators}

In this step, we have defined 124 quality indicators by combining base and derived measures, out of which 32 have been newly defined in our work. Usually, indicators are defined using the base or derived measures on a lower data model level, and they are themselves related to the higher data model levels. Similar as in the case of base and derived measures, an indicator can be related to different RDF data model levels. All the indicators are described in detail in the quality model wiki and are classified according to the quality characteristic that they measure.

From the previously specified derived measures, the following indicators were obtained:

- Average IRI dereferenceability. The average number of dereferenceable IRIs. This measure can be related to the triple, graph, or to the dataset levels and, furthermore, it is related to the Infrastructure aspect. 
- Average subject dereferenceability. The average number of dereferenceable subjects. This measure can be related to the graph or dataset levels and, furthermore, it is related to the Infrastructure aspect.

- Average disjoint classes. The average number of instances of disjoint classes. This measure can be related to the graph or dataset levels and, furthermore, it is related to the RDF model and Vocabulary aspects.

- Average domain consistency. The average number of triples in which the subject is consistent with the property domain. This measure can be related to the graph or dataset levels and, furthermore, it is related to the RDF model and Vocabulary aspects.

In some cases, quality indicators can be derived based only on base measures. An example of such indicators include:

- Instance interlinking. The average number of interlinked instances. This measure can be related to the graph or dataset levels and, furthermore, it is related to the Domain data aspect.

- Average short IRIs. The average number of short IRIs. This measure can be related to the graph or dataset levels and, furthermore, it is related to the $R D F$ model aspect.

All previously specified indicators have a ratio scale with values ranging from zero to one hundred, expressed in percentage.

Finally, some indicators have been directly defined without the need for base or derived measures. Examples of such indicators include

- SPARQL 1.1 support. Whether a dataset SPARQL endpoint supports the SPARQL 1.1 language. This indicator is related to the dataset level, and to the Infrastructure aspect. The possible values for this indicator are true and false.

The base measures, derived measures, and quality indicators in the Linked Data quality model are based on the results of the survey by Zaveri et al. [235], i.e., on the state of the art in Linked Data quality specification and assessment. However, the quality model proposed in this chapter describes a classification of measures in greater detail and it also defines a higher number of measures.

\subsubsection{Specification of Relationships Between Measures}

After the set of base measures, derived measures and indicators was defined, we have specified the formal relationships between these measures in terms of the formulas 
used for their calculation, which is a practice that is not always followed in the current state of the art.

Next, we present the formulas for the measures described in previous sections, in those cases in which a formalization through a formula applies.

The formula for Short IRI (4.1) defines whether an IRI is short with respect to some predefined threshold

$$
\text { IRI.length }<\text { threshold }
$$

Formulas for Number of dereferenceable IRIs (4.2) and Number of dereferenceable subjects (4.3) calculate the total number of dereferenceable IRIs and subjects in a dataset, respectively.

$$
\begin{gathered}
\text { \# different IRIs where (IRI dereferenceability = true) } \\
\text { \# triples where (Subject dereferenceability = true) }
\end{gathered}
$$

Formulas (4.4) and (4.5) calculate the Disjoint classes and Domain consistency derived measures, respectively.

$$
\begin{gathered}
\text { subject types } \nsubseteq \text { disjoint classes } \\
\text { subject types } \subseteq \text { property domain }
\end{gathered}
$$

Similarly as in the case of derived measures, the following formulas have been defined for indicators: Average IRI dereferenceability (4.6), Average subject dereferenceability (4.7), Average disjoint classes (4.8), Average domain consistency (4.9), Instance interlinking (4.10), and Average short IRIs (4.11).

$$
\begin{gathered}
\frac{\# \text { dereferenceable IRIs }}{\# \text { IRIs }} \times 100 \\
\frac{\# \text { dereferenceable subjects }}{\# \text { subjects }} \times 100 \\
\frac{\# \text { IRIs where }(\text { disjoint classes }=\text { true })}{\# \text { IRIs }} \times 100 \\
\frac{\# \text { triples where }(\text { domain consistency }=\text { true })}{\# \text { triples }} \times 100 \\
\frac{\# \text { interlinked subjects }}{\# \text { subjects }} \times 100
\end{gathered}
$$




$$
\frac{\text { \# IRIs where }(\text { Short IRI }=\text { true })}{\# \text { IRIs }} \times 100
$$

\subsubsection{Alignment with the Quality Model}

The last two steps of the followed bottom-up method suggest the definition of domain-specific quality sub-characteristics and their alignment with an existing quality model. In the case of Linked Data quality, as shown by Zaveri et al. [235], a large number of measures described in the survey are classified according to various dimensions. Therefore, for the Linked Data quality model we have decided to rely on the classification provided by Zaveri et al. and, starting from this classification and from the quality indicators identified in Section 4.2.2, we have identified the ISO 25012 quality characteristics that can be measured with the mentioned indicators.

The quality characteristics related to the indicators described in Section 4.2.2 include:

- Accessibility. The degree to which data can be accessed in a specific context of use, particularly by people who need supporting technology or special configuration because of some disability [114]. It can be measured using Average IRI dereferenceability and Average subject dereferenceability.

- Availability. The degree to which data has attributes that enable it to be retrieved by authorised users and/or applications in a specific context of use [114]. It can be measured using SPARQL 1.1 support.

- Completeness. The degree to which subject data associated with an entity have values for all expected attributes and related entity instances in a specific context of use [114]. It can be measured using Instance interlinking.

- Compliance. The degree to which data has attributes that adhere to standards, conventions or regulations in force and similar rules relating to data quality in a specific context of use [114]. It can be measured using Average short IRIs.

- Consistency. The degree to which data has attributes that are free from contradiction and are coherent with other data in a specific context of use [114]. It can be measured using Average disjoint classes and Average domain consistency.

The Linked Data quality model includes fifteen quality characteristics: accessibility, accuracy, availability, completeness, compliance, confidentiality, consistency, credibility, currentness, efficiency, precision, portability, recoverability, traceability, and understandability. Quality measures defined in the quality model cover twelve of 
Chapter 4. Linked Data Quality Model

these quality characteristics; as to this date no quality measures have been defined in the state of the art for three quality characteristics. The quality characteristics that do not have any quality measures associated are confidentiality, precision and recoverability.

Figure 4.3 presents the base measures, derived measures, indicators and quality characteristics presented as an example in this section, together with the references to the formulas described in Section 4.2.3. For a better visibility, some measures are repeated on the figure, and they are marked with the * sign. Due to space reasons, the inputs in the evaluation (disjoint classes and property domain) that are used for the calculation of some derived measures (i.e., Disjoint classes and Domain consistency) are not shown.

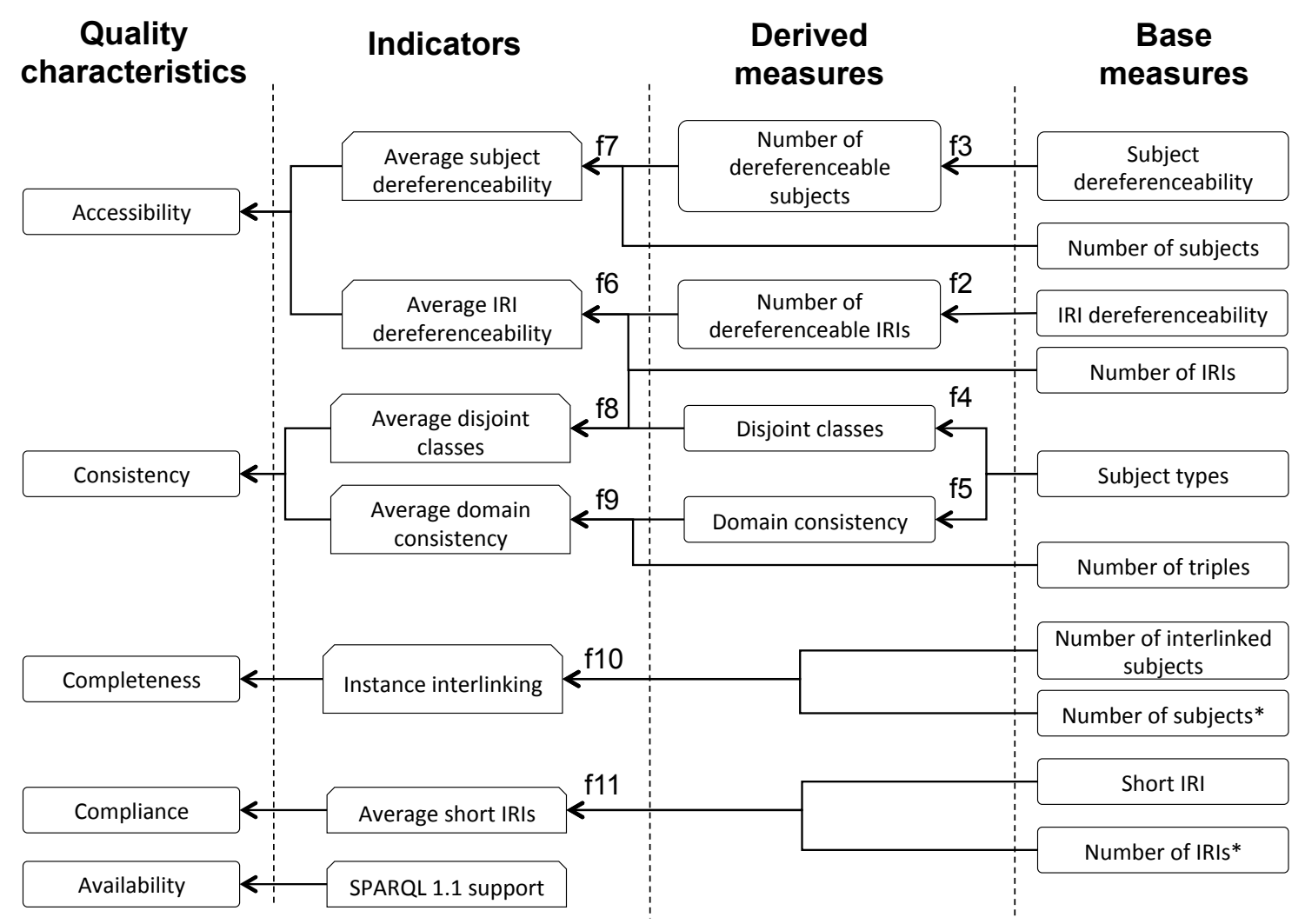

Figure 4.3: Subset of the Linked Data quality model

Table 4.1 shows all the quality characteristics identified in the Linked Data quality model, together with the indicators that can be used for their measurement. 
Table 4.1: Linked Data quality model characteristics and indicators.

\begin{tabular}{|c|c|}
\hline $\begin{array}{l}\text { Character. } \\
(\text { ISO } \\
25012)\end{array}$ & Indicators \\
\hline Accessibility & $\begin{array}{l}\text { Average IRI dereferenceability, Average subject dereferenceability, Average } \\
\text { predicate dereferenceability, Average object dereferenceability }\end{array}$ \\
\hline Accuracy & $\begin{array}{l}\text { Average automatic validation errors, Average crowdsourcing validation er- } \\
\text { rors, Average datatype syntax errors, Average syntactic rules syntax errors, } \\
\text { Average RDF pattern errors, Average ill-typed literals, Average datatype } \\
\text { compatibility, Average distance-based outliers, Average deviation-based } \\
\text { outliers, Average distribution-based outliers, Average triple correctness, } \\
\text { Average crowdsourced incorrect triples, Average misspelled literals, Av- } \\
\text { erage inaccurate labels, Average correct classification, Average property } \\
\text { discordance, Average invalid rules, Average entity mismatch }\end{array}$ \\
\hline Availability & $\begin{array}{l}\text { SPARQL support, SPARQL } 1.0 \text { support, SPARQL } 1.1 \text { support, RDF } \\
\text { dump, Average IRI RDF description, Average content type IRIs, Average } \\
\text { content negotiation support, Average accept header support, Average } \\
\text { sustainable IRIs, Multiple serialization formats, Multiple languages }\end{array}$ \\
\hline Completenes & $\begin{array}{l}\text { sInterlinking degree, Clustering coefficient, Centrality, Linked Data map- } \\
\text { pings, In-links, Average sameAs linked, Average blank nodes, Number of } \\
\text { entities, Number distinct properties, Average undefined classes, Average } \\
\text { undefined properties, Average undefined objects, Blank nodes use }\end{array}$ \\
\hline Compliance & $\begin{array}{l}\text { Average correct HTTP redirect, Average LDP GET support, Average } \\
\text { LDP PUT support, Machine-readable licence, Human-readable licence, } \\
\text { License propagation, Average HTTP IRIs, Average short IRIs, Average } \\
\text { IRI uniqueness }\end{array}$ \\
\hline Consistency & $\begin{array}{l}\text { Average stable IRIs, Average number of inconsistent functional dependence } \\
\text { subjects, Average disjoint classes, Average misplaced classes, Average mis- } \\
\text { placed properties, Average misused datatype properties, Average misused } \\
\text { object properties, Average deprecated subjects, Average deprecated proper- } \\
\text { ties, Average invalid inverse functional values, Average ontology hijacking, } \\
\text { Average negative dependent properties, Average geometric violation, Av- } \\
\text { erage domain consistency, Average range consistency, Average mapping } \\
\text { consistency, Average axiom violations, Schema completeness, Property } \\
\text { completeness, Population completeness, Instance interlinking, Average } \\
\text { mapped types }\end{array}$ \\
\hline
\end{tabular}

Table 4.1 - Continued on next page 
Table 4.1 - Continued from previous page

\begin{tabular}{|l|l|}
\hline $\begin{array}{l}\text { Character. } \\
\text { ISO } \\
\mathbf{2 5 0 1 2})\end{array}$ & Indicators \\
\hline Credibility & $\begin{array}{l}\text { Document digital signature, SPARQL digital signature, Average graph } \\
\text { digital signature, Author provenance, Contributors provenance, Publisher } \\
\text { provenance, Dataset sources provenance, Dataset ranking, Crowdsourcing } \\
\text { relevance, Provenance-based trust, Opinion-based thrust, Social networks } \\
\text { trust, Average facts trust, Blacklisted, Authority, Content-based trust, } \\
\text { Metadata-based trust, Average one-path trust, Average many-paths trust, } \\
\text { Decision network trust, List trust, Publisher trust, Association trust, } \\
\text { Average dataset rating }\end{array}$ \\
\hline Currentness & Dataset freshness, Datasource freshness \\
\hline Efficiency & $\begin{array}{l}\text { RDF dump compression, Average slash IRIs, Low latency, High through- } \\
\text { put, Response scalability, Average IRI caching, Average RDF primitives }\end{array}$ \\
\hline Portability & Terms reuse, Vocabulary reuse \\
\hline Traceability & Provenance \\
\hline $\begin{array}{l}\text { Understand- } \\
\text { ability }\end{array}$ & $\begin{array}{l}\text { SPARQL service description, Average internal redundant properties, Av- } \\
\text { erage external redundant properties, Average label unambiguity, Average } \\
\text { elements labelling, Dataset metadata, IRI pattern, Regular expression, } \\
\text { SPARQL examples, Vocabulary list, Mailing lists presence, Data inter- } \\
\text { pretability }\end{array}$ \\
\hline
\end{tabular}

\subsection{Ontological Representation of the Quality Model}

This section discusses how the quality model presented in the previous section can be represented in RDF using existing ontologies described in Section 4.1.3 and, furthermore, it presents a set of extensions to those existing ontologies so that the quality metrics and their measures can be described with fine-grained details.

\subsubsection{Conceptual Model}

Figure 4.4 shows the conceptual model of the ontology for representing the Linked Data quality model, using the terminology adopted in this chapter. The model describes a hierarchy of quality measures and quality characteristics related to a quality model, with important information such as measurement scales and scoring functions (i.e., the formulas for the calculation of values for a specific quality measure). Each quality measure is calculated using a specific technique (which can be automatic, semiautomatic or manual), it can be subjective or objective, has a specific duration, 
and can be used for obtaining some other measure. Furthermore, when performing an evaluation, a quality value related to a specific quality measure and an evaluation subject (e.g., a dataset) is obtained.

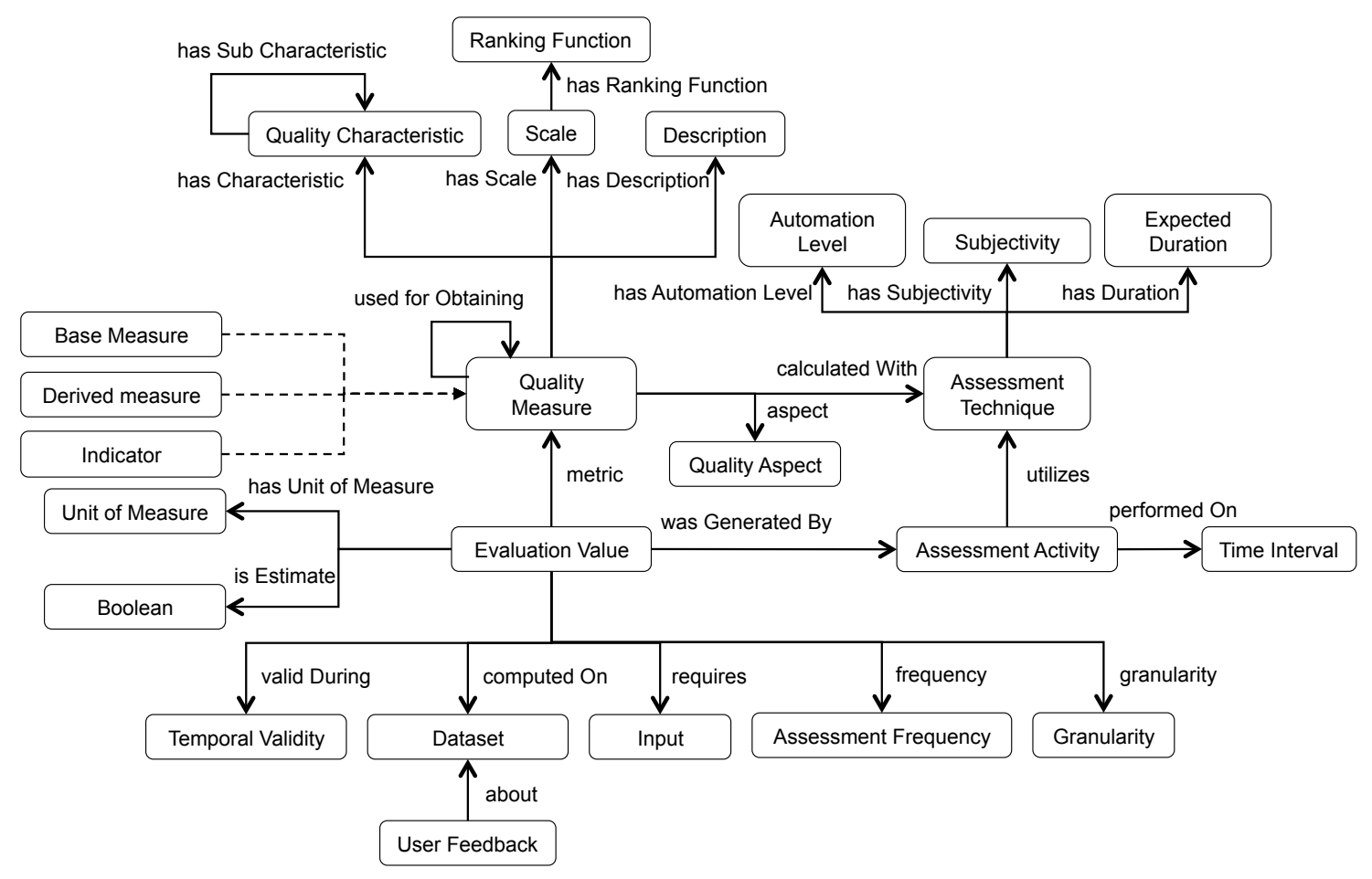

Figure 4.4: The conceptual model

\subsubsection{Extensions to Existing Ontologies}

The ontologies that could be used for the representation of quality specification and assessment of Linked Data, as described in Section 4.1.3, are either general or related to all types of data. For example, although DQV is a lightweight ontology that can suite the needs of Linked Data, it is defined to fit all types of data and it does not cover some aspects that are specific to Linked Data quality (Section 4.2). In this section we describe an extension of the existing ontologies, in order to cover the Linked Data specificities which is one of the requirements of our scenario of capturing quality-related information of Linked Data; this extension mainly relies on DQV, since it is expected to become the W3C standard for representing data quality.

Figures 4.5 and 4.6 present the proposed extension of the current ontologies for quality representation and assessment, adapted to the domain of Linked Data. The classes already described in the existing ontologies (Section 4.1.3) are presented in 
white boxes, together with their namespaces, while the extensions are represented with grey boxes; new properties are marked in bold.

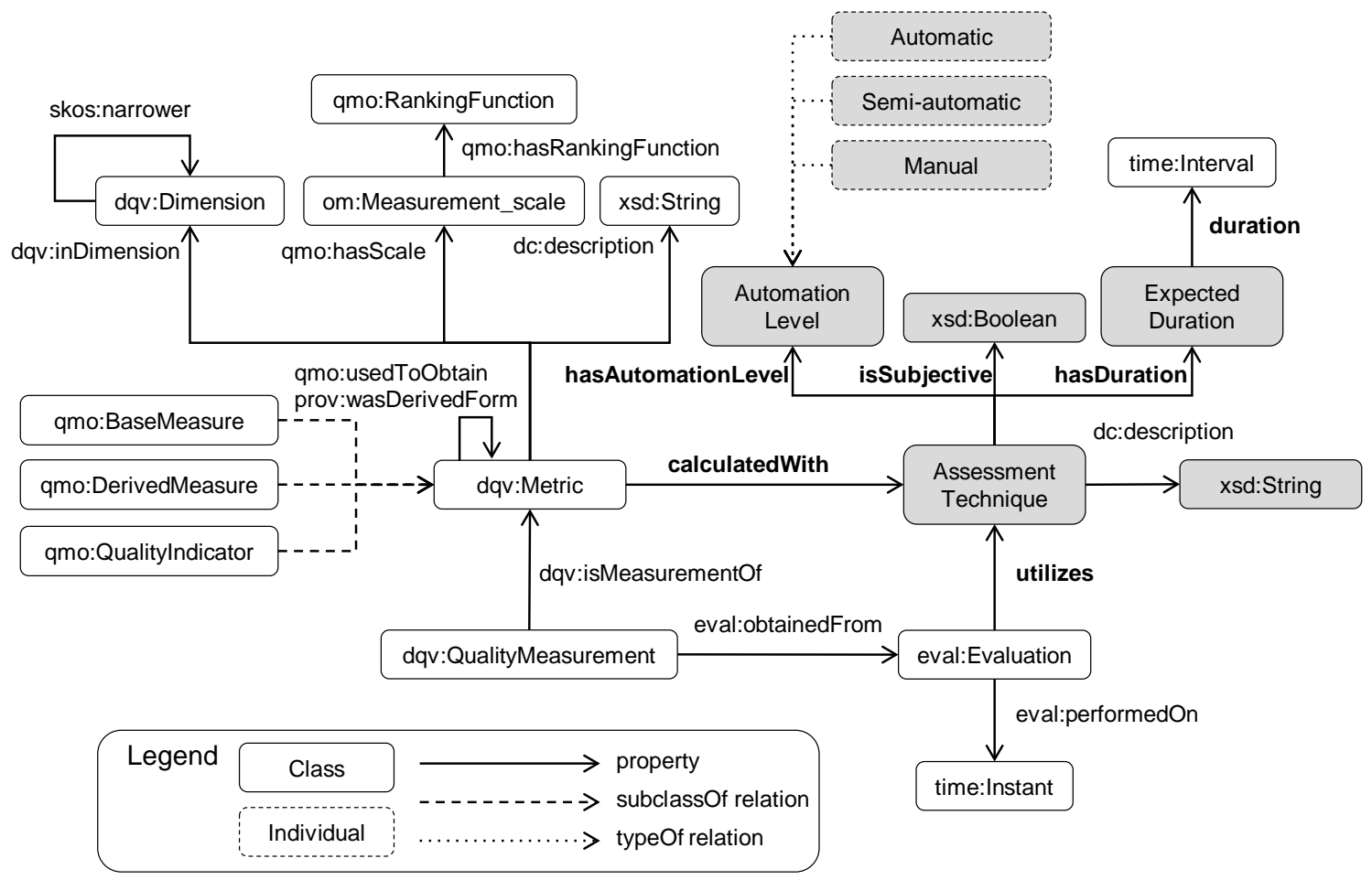

Figure 4.5: The ontology extension (I)

Quality values obtained in an evaluation are represented with the dqv:QualityMeasurement class, with equivalent classes being eval:QualityValue and daq:Observation. Quality measures are represented with the dqv:Metric class, with equivalent classes being qmo:QualityMeasure and daq:Metric. Furthermore, QMO classes representing base measures, derived measures and quality indicators are also reused. Quality characteristics are represented with the dqv:Dimension class, with equivalent classes being qmo:QualityCharacteristic and daq:Dimension. Evaluation processes are represented with the eval:Evaluation class, while datasets are represented with the dcat:Dataset class from the well-known DCAT ontology ${ }^{6}$. Furthermore, some classes for representing concepts such as time instants, time intervals, measurement scales, and units of measurement are reused from the W3C Time ontology ${ }^{7}$ (time) and from the ontology of units of measure ${ }^{8}(\mathrm{om})$.

\footnotetext{
${ }^{6}$ http: //www.w3.org/TR/vocab-dcat/

${ }^{7}$ http://www.w3.org/TR/owl - time/

${ }^{8}$ http://WwW. wurvoc.org/vocabularies/om-1.8/
} 
By extending the $\mathrm{QMO}$ ontology, relationships between quality measures are incorporated into the proposed extensions. QMO defines relationships which denote that one measure can be used for obtaining some other measure, or that increasing the value of one measure implies increasing or decreasing the value of some other measure.

In some cases for certain measures and their scales a qmo:RankingFunction property can be specified in an objective way, denoting whether in the case of numerical results obtained for such measures higher or lower values are more desirable (e.g., for precision or number of dereferenceable URIs, it is clear that a higher value is more desirable).

The extensions of the presented ontology tend to cover additional information related to Linked Data quality specification and assessment which can be important for easier interpretation, benchmarking, interchange, and understanding of quality measures and evaluation results. The QualityAspect class describes the aspect of Linked Data quality the measure is related to (Figure 4.1), while Granularity class describes the RDF level (Figure 4.2) related to concrete value obtained in the evaluation (e.g., the value of 0.85 for precision measure is obtained for a graph $g$ ) or to the quality measure (e.g., precision quality measure can be calculated for graphs or datasets). These two concepts are specifically related to Linked Data quality. The motivation for including these two concepts is to allow quality evaluators to select and filter the most relevant and applicable metrics depending on their use case. For instance, if there was a change in the infrastructure or in the transformation process, evaluators can select the aspects that have the most impact from those changes and re-evaluate them. Similarly, depending on whether one is evaluating a single triple, a graph, or a dataset she can select the most relevant metrics using the granularity concept.

Some extensions, although could be related to data quality in general, could also carry valuable information for Linked Data. These include the information on how often a measure has to be assessed (AssessmentFrequency), whether a measure is dependent on the system (isSystemDependent), the period of time during which the result for the measure is valid (TemporalValidity), the technique used in the evaluation for assessing a measure (AssessmentTechnique), whether an assessment technique is subjective or objective (isSubjective), the expected duration of the assessment (ExpectedDuration), and whether an evaluation is done automatically, semi-automatically or manually (AutomationLevel). 


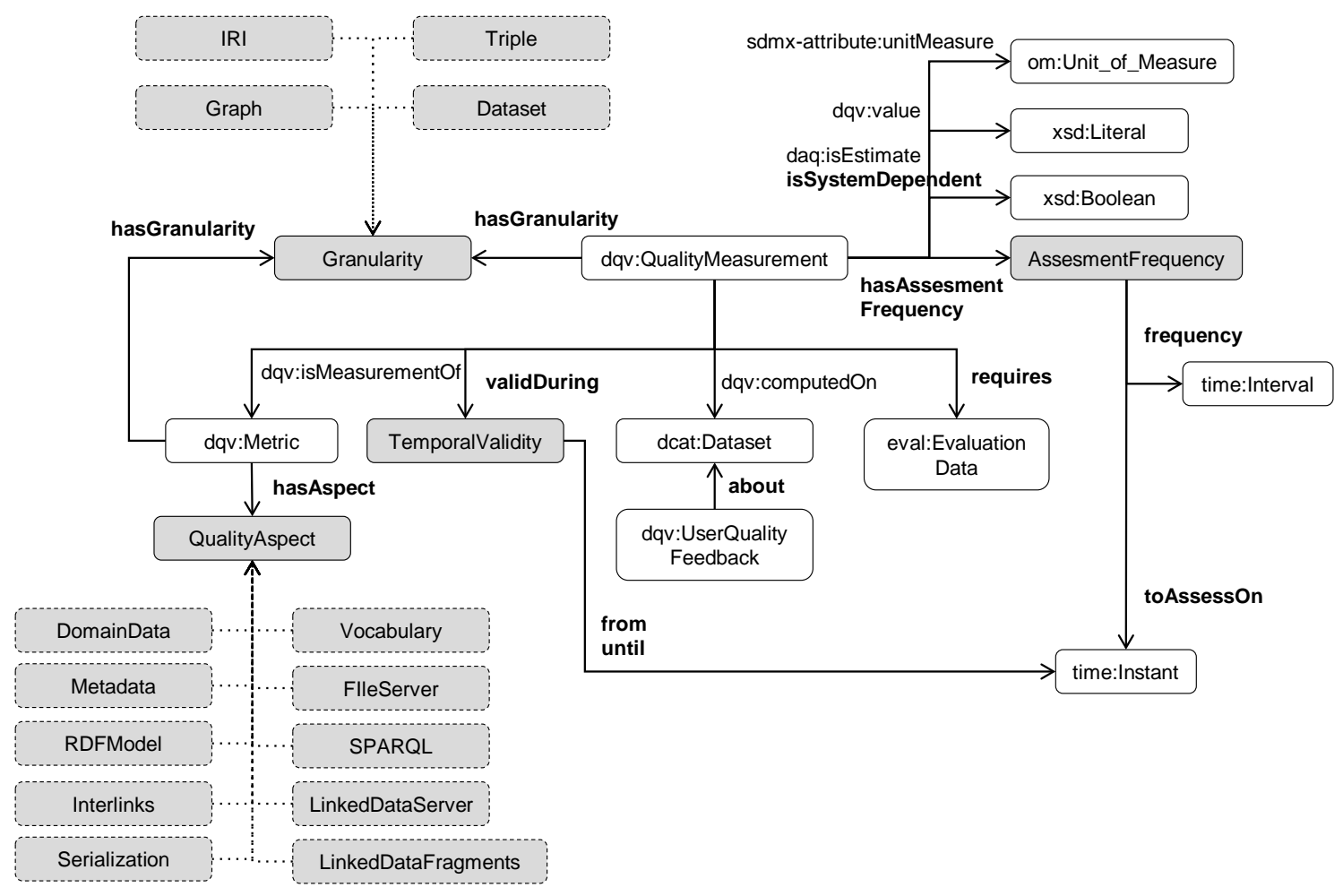

Figure 4.6: The ontology extension (II)

\subsubsection{Representing the Linked Data Quality Model}

The ontology presented in the previous section has been implemented in OWL and is available online ${ }^{9}$.

The Linked Data quality model presented in this chapter has been described in RDF by using the ontology extension. The description is available online ${ }^{10}$ and it can be reused by the tools that utilise the quality model presented in this chapter, which can bring consistency in results representation across various evaluation efforts. To this extent, the provided RDF representation of the Linked Data quality model can be significant in the production, interchange, and consumption of quality evaluation data.

\footnotetext{
${ }^{9}$ http: //www. linkeddata.es/ontology/ldq/

${ }^{10}$ http ://linkeddata.es/resource/ldqm/
} 


\subsection{LD Sniffer}

$L D$-Sniffer is a tool that was developed to verify the feasibility of using the Linked Data quality model guiding Linked Data quality assessment tool development, specification of quality assessment requirements, and representation of quality assessment results. The LD Sniffer tool ${ }^{11}$ was developed as an open source project under the Apache 2.0 license $^{12}$.

The LD-sniffer tool evaluates the accessibility of Linked Data resources according to the metrics defined in the quality model. Given a set of linked data URIs, $L D$ Sniffer makes HTTP requests to each of the URIs that are in it and evaluates the responses to calculate quality metrics. LD-Sniffer can detect URIs that are no longer alive and URIs that do not meet the requirements of web standards and linked data standards. Figure 4.7 shows the web interface of LD-Sniffer.

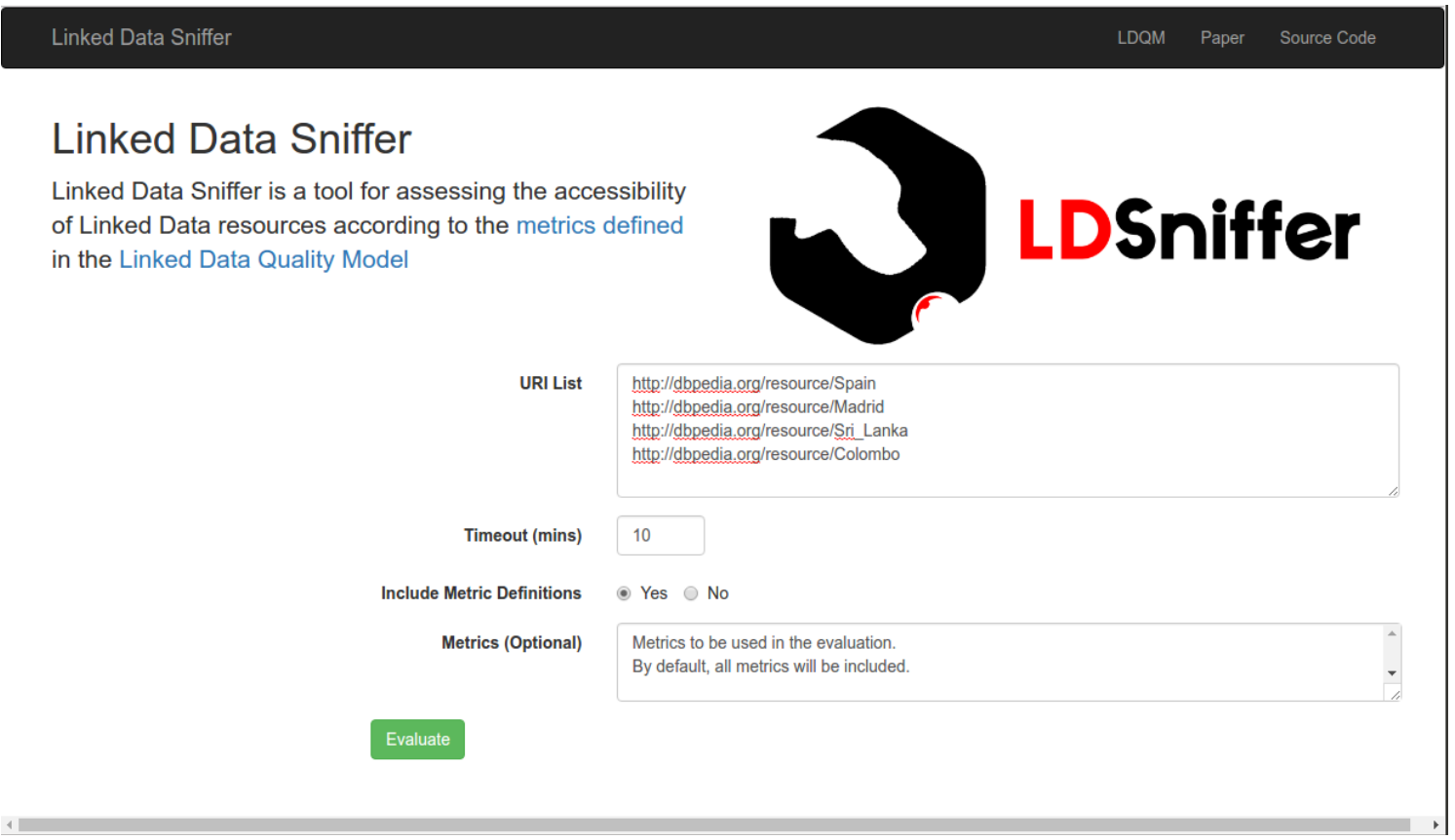

Figure 4.7: LD Sniffer web interface

LD Sniffer is also provides a command line interface (also available as a docker image) that can be used to run batch jobs for a large-scale evaluation when accessibility of thousands of Linked Data IRIs has to be checked. Listing 4.1 shows the parameters of the command line application and Figure 4.8 illustrates an image of the docker

\footnotetext{
${ }^{11}$ https://github.com/nandana/ld - sniffer

${ }^{12}$ https : //Www . apache. org/licenses/LICENSE- 2.0
} 
container. A large scale evaluation of $100 \mathrm{~K}$ resources in DBpedia performed using LD Sniffer is presented in Section 8.1.2.

Listing 4.1: LD Sniffer command line options
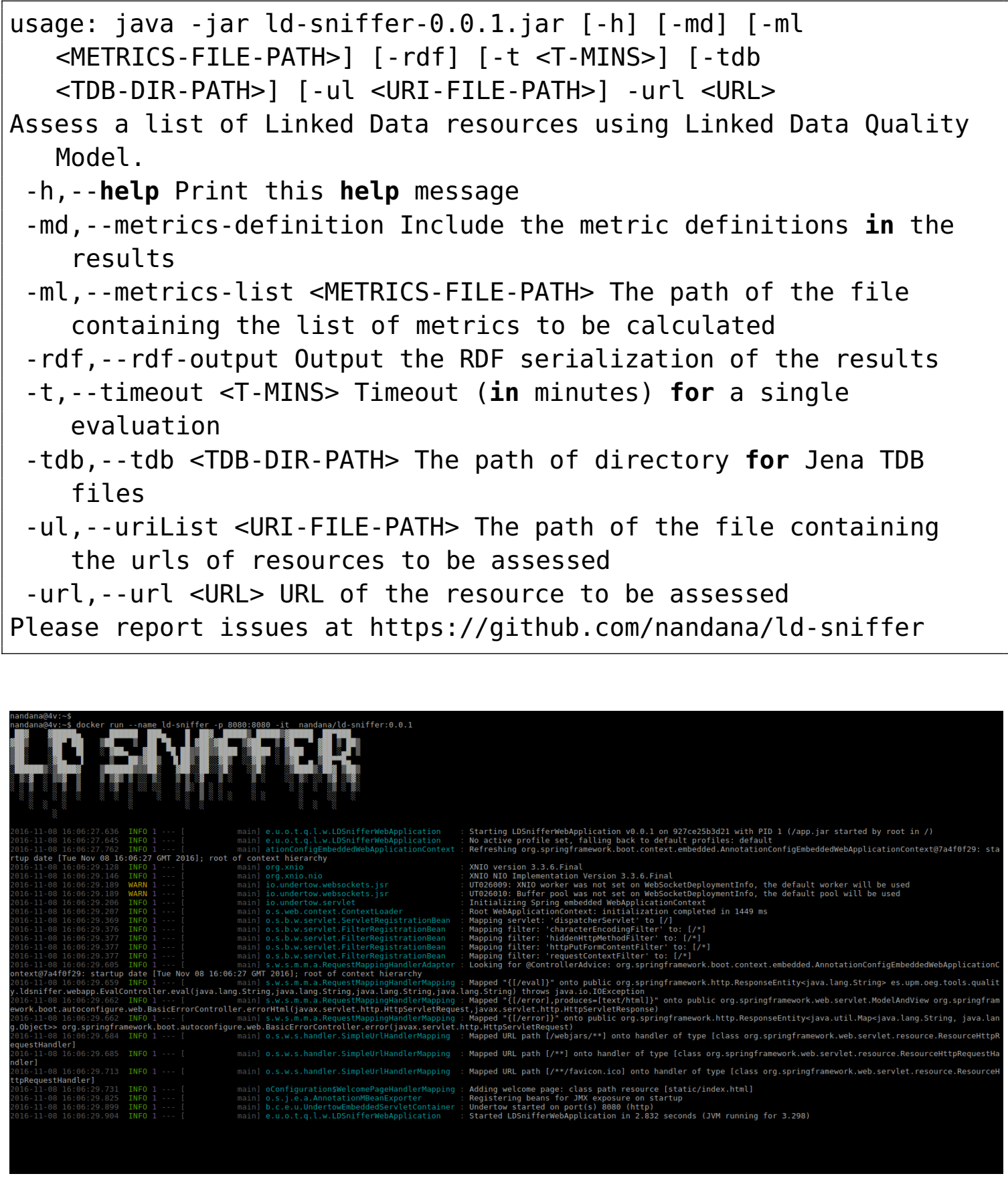

Figure 4.8: LD Sniffer docker container 


\subsection{An Example}

This section provides few examples of evaluation results and quality measure definitions following the ontology extensions presented in Figure 4.5 and Figure 4.5. Furthermore, it provides an example on how to query such quality assessment results.

Listing 4.2 shows an example quality assessment result for evaluating average subject dereferenceability measure for DBpedia dataset. It provides provenance metadata on the evaluation activity, what techniques were utilised, the corresponding quality aspect, unit measures for the value, scale and other information.

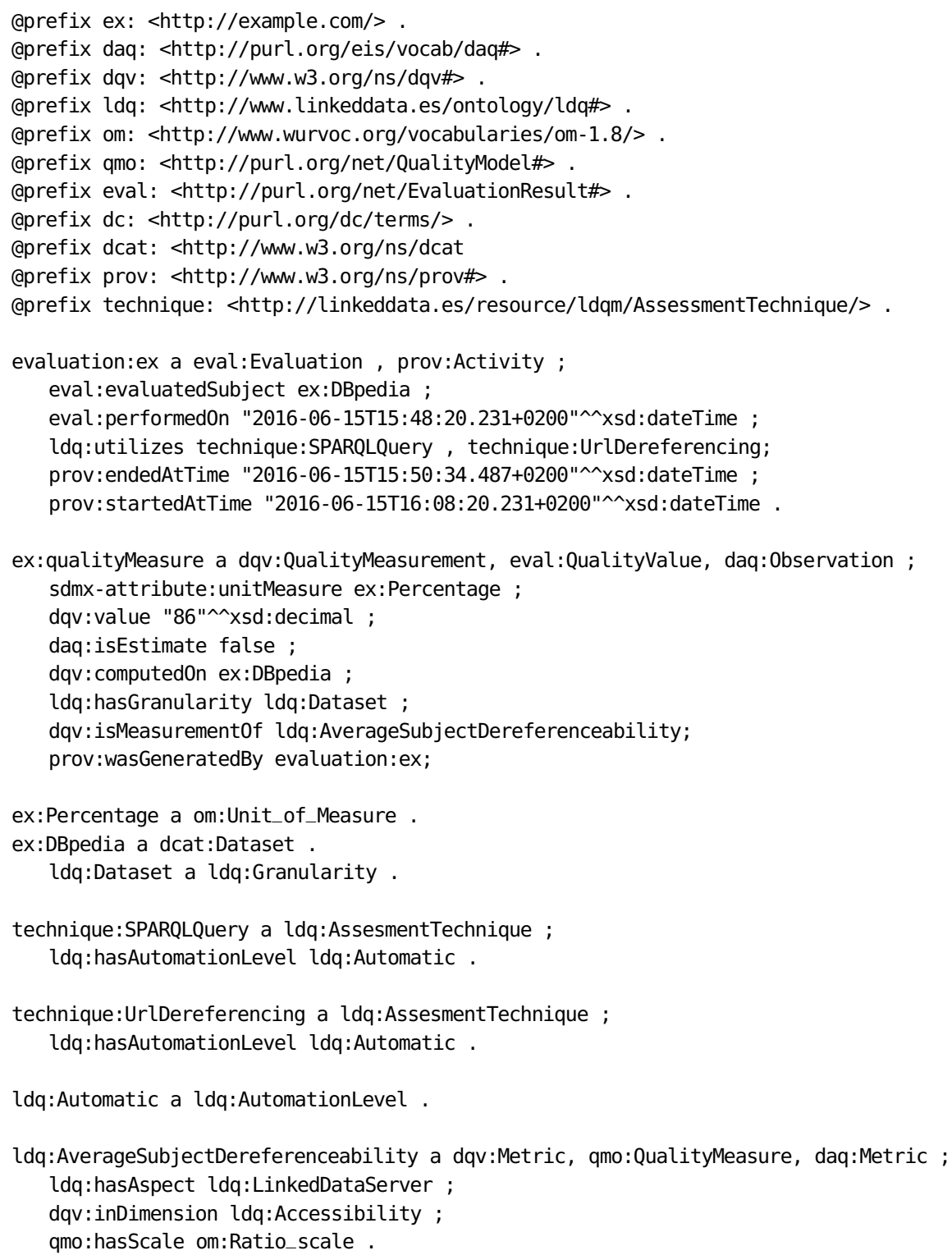




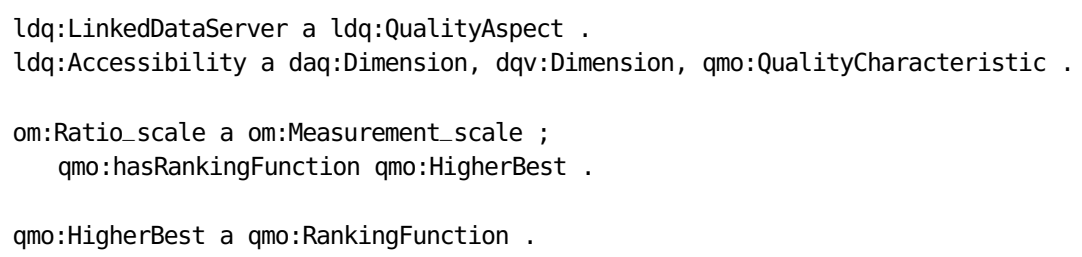

Listing 4.2: An example quality assessment result

Listing 4.3 shows a query that illustrates the means for capturing all measures that can be used for the evaluation of RDF triples. The fact that evaluation results are represented in RDF using unified terminology makes it easier consumer the results using queries. Furthermore, the evaluation results from different evaluation campaigns can be easily merged and perform comparison queries.

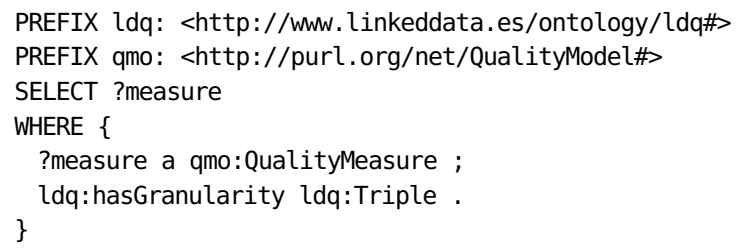

Listing 4.3: SPARQL query I

Similarly, Listing 4.4 illustrates the means for finding datasets that have average subject dereferenceability $100 \%$. Such queries can help to filter datasets that fulfill a certain quality criterion.

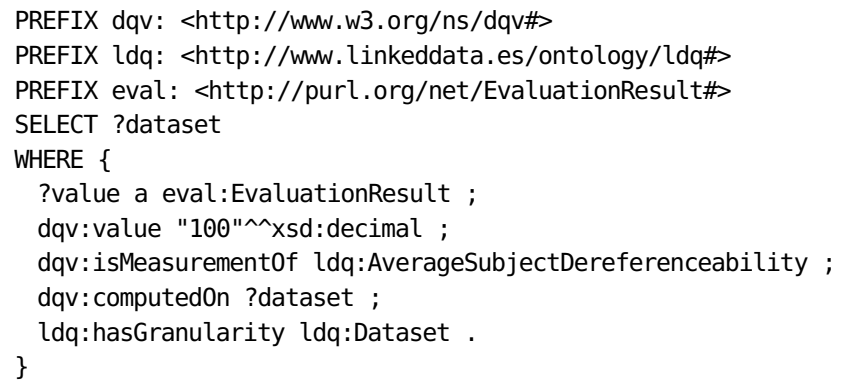

Listing 4.4: SPARQL query II

Finally, the example query in Listing 7.6 illustrates the means for capturing evaluation values for average subject dereferenceability for a specific dataset, that are obtained in different evaluations. Such queries can be useful for generating quality dashboards that show specific quality measures of a given dataset. 


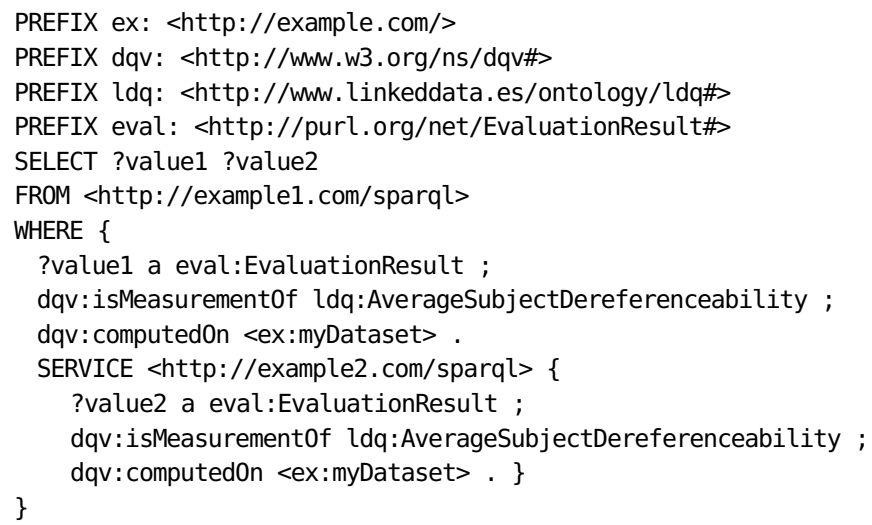

Listing 4.5: SPARQL query III.

\subsection{Future Work}

The quality requirements of different use cases evolve, thus the quality model has to be updated regularly with the new quality measures to keep the quality model up-to-date.

Furthermore, one of the uses of the quality model was to guide the Linked Data publishers which quality measures and indicators are important in different use cases. To this end, potential future work can be twofold. On the one hand, user studies can be carried out to identify the perceived importance of measures and indicators by relevant actors of different use cases. On the other hand, measures and indicators can be grouped to different profiles by their importance to different use cases. 


\section{Chapter 5}

\section{Linked Data Profiling Framework}

As discussed in Chapter 3, the second objective of this thesis is to improve RDF data profiling techniques focusing on Linked Data quality assessment and repair use cases. The goal is to extend the current RDF data profiling techniques to include the fine-grained profiling metrics that is needed for quality assessment and to propose an extended RDF dataset description model for representing data profiling metrics such as dataset statistics, and other dataset pattern information with the level of detail required by quality assessment and repair techniques. This chapter presents the Linked Data profiling framework proposed in this thesis.

The Linked Data principles promote publishing data in a machine-readable manner using Web standards and interlinking them. The Linked (Open) Data cloud has grown at a rapid rate in recent years providing a vast amount of valuable data from many different domains such as life sciences, government data, geographical data, media and publications. Nevertheless, for data to be useful, data consumers should be able to discover, understand, and query such data. Furthermore, the data should have a sufficient level of quality for a given use case, i.e., data is "fit for use". Metadata (i.e., data about data) plays an essential role in making data discoverable, understandable, and metadata can provide useful indications about data quality. Data profiling is one common technique that can be used to generate valuable metadata about a given dataset.

Dataset metadata can be categorised into two groups based on the way they are generated: manual metadata and analytical metadata. On the one hand, manual metadata is the type of metadata that can not be automatically generated because it needs human intervention to know the values of such metadata. For instance, provenance metadata such as the creator of a dataset or access metadata such as the license of a given dataset can not be automatically generated when they are not known in advance or not explicitly present in data themselves. On the other hand, analytical metadata is the type of metadata that can be automatically generated by 
analysing the data content, its structure, or other aspects that can be easily measured. For instance, generic statistics such as the number of entities described in data or the distribution of a particular set of values can be easily extracted automatically. In the framework presented in this chapter, we limit our scope to analytical metadata and discuss which types of metadata can be generated by data profiling techniques.

Data profiling for the relational data is defined as "the activity of creating small but informative summaries of a database" [126]. According to such definition, those summaries could include both simple statistics such as the number of records in a given table or in the whole database as well as information derived from complex statistics such as functional dependencies in data or the type of distribution of values in a given column of a table. In more general terms, data profiling is defined as "the set of activities and processes to determine the metadata about a given dataset" [3]. This chapter discusses Linked Data profiling and the models that can represent the information generated by data profiling.

Data profiling is a widely-used technique in the relational data domain. There are a large number of popular commercial tools for profiling relational data, for example, IBM's InfoSphere Information Analyzer ${ }^{1}$ or Microsoft's SQL Server Integration Services (SSIS) ${ }^{2}$.

Even though there are several RDF data profiling tools and vocabularies for representing profiling metrics exists, we do not observe data profiling being widely used similarly as in the relational data domain. More specifically, we argue that data profiling is under-utilised for quality assessment and repair of Linked Data. In her Ph.D. thesis, Spahiu concludes that the current profiling tools are limited in reporting some basic statistics after thoroughly analysing the state-of-the-art tools in Linked Data profiling [213]. Our hypothesis is that the current data profiling techniques lack the expressivity to represent the fine-grained details necessary for data quality assessment and repair use cases and this is hindering the use for data quality.

In this chapter, we first analyse the use cases for Linked Data profiling (Section 5.1) and identify profiling tasks specific to Linked Data represented using the RDF model (Section 5.2). Then, Loupe, a novel Linked Data profiling framework is presented which consists of (a) an extended dataset description model, (b) a Linked Data profiling toolkit, (c) the Loupe Web portal, and d) the Loupe API (Section 5.4). The Loupe framework has been used in several practical use case scenarios and those cases are discussed in Section 9.3. Finally, some future directions for the Loupe Linked Data profiling framework are discussed in Section 5.5.

The Linked Data Profiling framework provides inputs to achieve the main goal of this thesis, that is to improve the techniques for quality assessment and repair.

\footnotetext{
${ }^{1}$ https://WwW.ibm. com/us - en/marketplace/infosphere-information-analyzer

${ }^{2}$ https : //docs . microsoft . com/es - es/sql/integration-services/sql - server integration-services
} 
Chapter 5. Linked Data Profiling Framework

Chapter 6 discusses how the profiling metrics generated by the Loupe Linked Data profiling framework is used to learn RDF Shapes and Chapter 7 discusses how such information is used to perform quality assessment and repair of Linked Data.

\subsection{Linked Data Profiling Use Cases}

Even though the work presented in this thesis is focused on quality assessment and repair techniques, data profiling can produce valuable analytical metadata that can be used in a wide variety of practical use cases. These use cases have been collected both from exiting literature as well as from the authors' experience based on the requirements of the use cases where the proposed framework was already utilised. The use cases that were found in literature include schema discovery [28, 37], data visualisation [149], data summarization [133], distributed query planning and optimisation [28, 82, 123], data integration and linking [28, 204], outlier detection [80], and ontology re-engineering [2].

Table 5.1 lists a non-exhaustive list of potential use cases for Linked Data profiling. In order to have a better use case categorisation, we have grouped the use cases into more higher-level use case categories compared to some of the concrete cases presented in the aforementioned literature. For instance, the ontology re-engineering use case [2] is related to studying the ontology misuse by datasets including the presence of over-specified classes (i.e. rarely used in real-world data). We have included a category vocabulary usage and adoption monitoring to cover this use case and other possible use cases related to understanding vocabulary adoption.

Table 5.1: Linked Data profiling use cases

\begin{tabular}{|l|l|l|}
\hline ID & $\begin{array}{l}\text { Use case cate- } \\
\text { gory }\end{array}$ & Role of data profiling \\
\hline U1 & Data exploration & $\begin{array}{l}\text { To provide concise and useful information about } \\
\text { the content and internal structure of a dataset, } \\
\text { making it easier for consumers to understand data. }\end{array}$ \\
\hline U2 & $\begin{array}{l}\text { Data search and } \\
\text { discovery }\end{array}$ & $\begin{array}{l}\text { To build indices that can allow search and discovery } \\
\text { of data. }\end{array}$ \\
\hline U3 & $\begin{array}{l}\text { Data summariza- } \\
\text { tion }\end{array}$ & $\begin{array}{l}\text { To generate descriptive and informative summaries } \\
\text { of large datasets. }\end{array}$ \\
\hline U4 & $\begin{array}{l}\text { Data-driven } \\
\text { tion }\end{array}$ & $\begin{array}{l}\text { To find common patterns in data and to obtain a } \\
\text { schema that in present in data. }\end{array}$ \\
\hline
\end{tabular}

Table 5.1 - Continued on next page 
Table 5.1 - Continued from previous page

\begin{tabular}{|c|c|c|}
\hline ID & $\begin{array}{l}\text { Use case cate- } \\
\text { gory }\end{array}$ & Role of data profiling \\
\hline U5 & $\begin{array}{l}\text { Data quality } \\
\text { assessment and } \\
\text { cleansing }\end{array}$ & $\begin{array}{l}\text { To activities such as outlier detection and auto- } \\
\text { matic validation rules generation. }\end{array}$ \\
\hline U6 & $\begin{array}{l}\text { Data integration } \\
\text { and linking }\end{array}$ & $\begin{array}{l}\text { To gather knowledge about structure and content } \\
\text { of heterogeneous data sources and also to identify } \\
\text { overlaps and duplicates. }\end{array}$ \\
\hline U7 & $\begin{array}{l}\text { Instance-based } \\
\text { ontology match- } \\
\text { ing }\end{array}$ & $\begin{array}{l}\text { To match two ontologies by analysing the patterns } \\
\text { seen in data. }\end{array}$ \\
\hline U8 & $\begin{array}{l}\text { Query optimisa- } \\
\text { tion }\end{array}$ & $\begin{array}{l}\text { To query optimisation algorithms that will rear- } \\
\text { range the different actions in the query plan to } \\
\text { optimise the resources. }\end{array}$ \\
\hline U9 & $\begin{array}{l}\text { Vocabulary us- } \\
\text { age and adoption } \\
\text { monitoring }\end{array}$ & $\begin{array}{l}\text { To provide useful insights about which parts of } \\
\text { vocabularies are actually being used in data. }\end{array}$ \\
\hline U10 & $\begin{array}{l}\text { Data visualisa- } \\
\text { tion }\end{array}$ & $\begin{array}{l}\text { To provide quick access to aggregated data for } \\
\text { visualisation rather than extracting then on the fly } \\
\text { and performing calculations. }\end{array}$ \\
\hline U11 & $\begin{array}{l}\text { Monitoring } \\
\text { dataset changes }\end{array}$ & $\begin{array}{l}\text { To monitor changes in datasets and also identify } \\
\text { undesired states caused by accidental or malicious } \\
\text { actions. }\end{array}$ \\
\hline U12 & $\begin{array}{l}\text { Topic domain } \\
\text { discovery }\end{array}$ & $\begin{array}{l}\text { To identify the main domain or topic of a dataset } \\
\text { automatically; such topics could support tasks such } \\
\text { as dataset classification, or contextual search. }\end{array}$ \\
\hline U13 & $\begin{array}{l}\text { Data compres- } \\
\text { sion }\end{array}$ & $\begin{array}{l}\text { To identify the best strategies for compressing a } \\
\text { dataset based on its structure and to predict the } \\
\text { possible levels of compression. }\end{array}$ \\
\hline
\end{tabular}

\subsection{Linked Data Profiling Tasks}

Having discussed the Linked Data profiling use cases, in this section, we identify concrete profiling tasks and metrics that are relevant to Linked Data profiling. Data profiling can consist of many different tasks depending on (a) the type of data being profiled and (b) the purpose or the use case in which the profiling metrics will be used.

For instance, in relational data, the tasks influenced by the tabular structure of 
Chapter 5. Linked Data Profiling Framework

relational data and are generally classified into three main categories: single column analysis, multiple column analysis, and dependency analysis [3]. Single column analysis consists of analysing the values of an individual column for tasks such as checking the number of unique values, the number of null values, value distributions, value ranges, common patterns, etc. Multiple column analysis consists of tasks such as discovering correlations and association rules among different columns or clustering of similar columns. Dependency analysis consists of tasks such as identification of columns with key values or identification of foreign keys.

Spahiu et al. identify three paradigms for Linked Data profiling: (a) schemabased Profiling, (b) topic-based Profiling, and (c) linkage-based profiling [213]. In schema-based profiling, the profiling is focused on the structure and the schema of data and generate metadata related to those aspects that could help in use cases such as validating consistency or error detection. Topic-based profiling is more related to the content of the data and focused on identifying the topic of data; such profiling is useful for dataset classification and labelling and contextual search. Linkage-based profiling is focused on the links between different entities and is related to use cases, such as instance matching or detecting similarities between entities. Because the goal of this work is to improve data profiling techniques to be utilised in data quality assessment and repair, we focus on schema-based profiling.

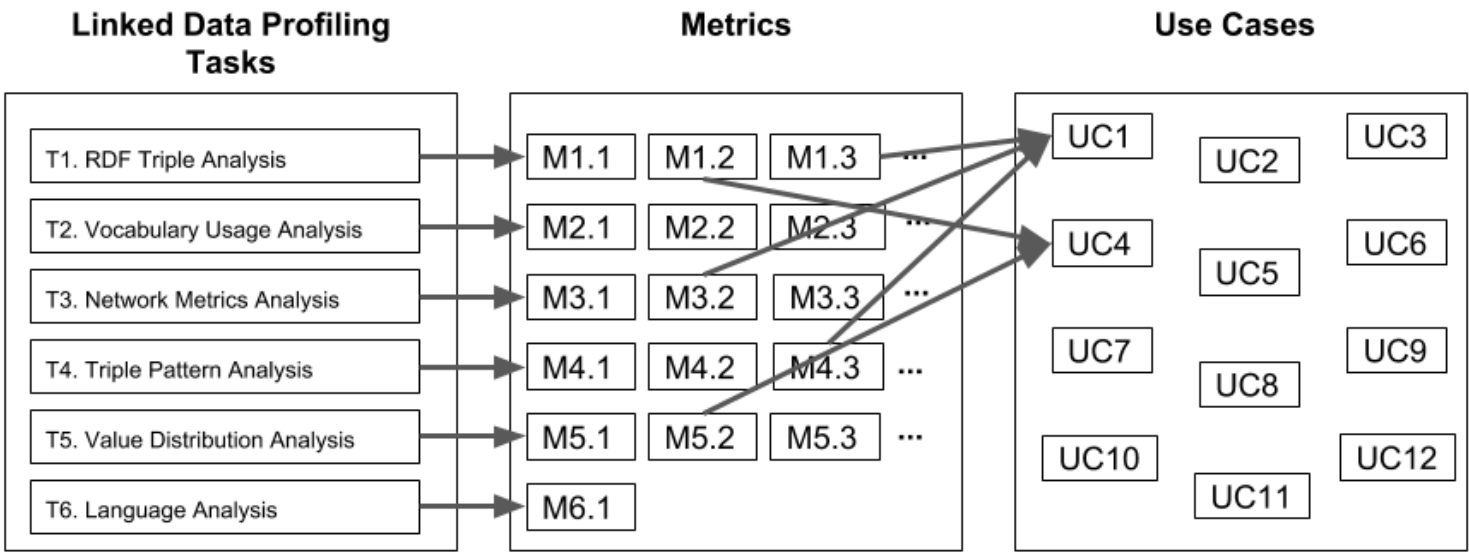

Figure 5.1: Relationship between Linked Data profiling tasks, metrics, and use cases

To this end, we identify six different categories for schema-based Linked Data profiling:

- T1. RDF Triple Analysis

- T2. Vocabulary Usage Analysis 
- T3. Network Metrics Analysis

- T4. Triple Pattern Analysis

- T5. Value Distribution Analysis

- T6. Language Analysis

The following sections identify concrete Linked Data profiling metrics related to each of the profiling tasks. A combination of such metrics is used in each of the aforementioned Linked Data profiling use case categories (Table 5.1) as illustrated in Figure 5.1.

\subsubsection{RDF Triple Analysis (T1)}

In the RDF Triple Analysis task, the triples in an RDF dataset are inspected only considering the semantics related to the RDF model to define a set of metrics related to the task. In this task, the analysis is performed without taking into account the vocabularies used and other aspects related to vocabularies or ontologies used in the dataset. The different metrics are derived by considering the concepts defined in the RDF data model. The main structure considered in this analysis is the triple which encodes a statement and consists of a subject, a predicate, and an object. Each of these allows different types of elements: a subject can be either an IRI or a blank node; a predicate is an IRI, and an object can be an IRI, a blank node, or a literal [52]. An RDF graph is a set of triples and can have an associated IRI or a blank node as its graph name (i.e., named graphs). It is important to note that RDF graphs can have duplicate triples. An RDF dataset is a set of RDF graphs [52].

More formally, elements in the RDF model can be defined following the work by Muñoz et al [177]. If we consider three pairwise disjoint infinite sets U, B, L which denote IRIs, blank nodes, and literals respectively, an RDF triple, $t$, can be defined as $t=\langle s, p, o\rangle \in\langle U \cup B\rangle \times\langle U\rangle \times\langle U \cup B \cup L\rangle$. In the triple, $s, p$, o refer to the subject, the predicate, and the object respectively. An RDF graph, $G_{i}$, is a set of RDF triples, i.e., $\left\langle s_{i}, p_{i}, o_{i}\right\rangle \in G_{i}$. An RDF dataset, $D_{i}$, is a set of RDF graphs, i.e., $g \in D_{i}$.

When the set of all the subjects, predicates, or objects in an RDF graph is considered, one can count either the total count (including duplicates) and the distinct count (only considering unique values). Two IRIs are considered equal if they are equivalent under Simple String Comparison as defined in RFC3987 [66]. Two literals are considered equal if and only if the two lexical forms, the two datatype IRIs, and the two language tags (if there is any) compare equal, character by character. The RDF 1.1 specification does not define the structure of blank nodes; thus, their equality depends on the RDF store implementations. 
Chapter 5. Linked Data Profiling Framework

Table 5.2: RDF triple analysis (T1)

\begin{tabular}{|c|c|c|}
\hline ID & Name & Description \\
\hline \multicolumn{3}{|c|}{ M1.1 - Dataset Size } \\
\hline M1.1.1 & datasetTripleCount & The number of triples in the dataset. \\
\hline M1.1.2 & duplicateTripleCount & The number of duplicate. \\
\hline \multicolumn{3}{|c|}{ M1.2 - Named Graph Information } \\
\hline M1.2.1 & namedGraphURI & $\begin{array}{l}\text { The IRI that identifies each distinct named } \\
\text { graph. }\end{array}$ \\
\hline M1.2.2 & namedGraphTripleCount & $\begin{array}{l}\text { The number of triples in the given named } \\
\text { graph. }\end{array}$ \\
\hline \multicolumn{3}{|c|}{ M1.3 - Subject Counts } \\
\hline M1.3.1 & distinctSubjectCount & The number of distinct subjects. \\
\hline M1.3.2 & iriSubjectCount & The number of IRI subjects. \\
\hline M1.3.3 & distinctIriSubjectCount & The number of distinct IRI subjects. \\
\hline M1.3.4 & blankNodeSubjectCount & The number of blank node subjects. \\
\hline M1.3.5 & $\begin{array}{l}\text { distinctBlankNodeSubject } \\
\text { Count }\end{array}$ & The number of distinct blank node subjects. \\
\hline \multicolumn{3}{|c|}{ M1.4 - Object Counts } \\
\hline M1.4.1 & distinctObjectCount & The number of distinct objects. \\
\hline M1.4.2 & iriObjectCount & The number of IRI objects. \\
\hline M1.4.3 & distinctIriObjectCount & The number of distinct IRI objects. \\
\hline M1.4.4 & blankNodeObjectCount & The number of blank node objects. \\
\hline M1.4.5 & $\begin{array}{l}\text { distinctBlankNodeObject } \\
\text { Count }\end{array}$ & The number of distinct blank node objects. \\
\hline M1.4.6 & literalObjectCount & The number of literal objects. \\
\hline M1.4.7 & $\begin{array}{l}\text { distinctLiteralObject } \\
\text { Count }\end{array}$ & The number of distinct literal objects. \\
\hline \multicolumn{3}{|c|}{ M1.5 - Distinct Node Counts } \\
\hline M1.5.1 & totalRDFNode & The total number of distinct RDF nodes. \\
\hline M1.5.2 & totallRIs & $\begin{array}{l}\text { The total number of IRIs as either subject, } \\
\text { predicate, or object }\end{array}$ \\
\hline M1.5.3 & totalBlankNodes & $\begin{array}{l}\text { The total number of blank nodes as either } \\
\text { subject, predicate, or object }\end{array}$ \\
\hline
\end{tabular}

With this background, the metrics extracted in this analysis include: information about dataset size, information about subjects, and objects. The subjects can be further analysed to extract the absolute and distinct counts of IRIs, and blank nodes. Similarly, the objects can be analysed to extract absolute and distinct counts of IRIs, blank nodes, and literals. The list of all the profiling metrics related to T1 is 
presented in Table 5.4.

These metrics are useful in data exploration (U1), summarisation (U4), monitoring dataset changes (U11), and query optimisation (U8).

\subsubsection{Vocabulary Usage Analysis (T2)}

The vocabulary usage analysis task studies how different vocabularies are used in a dataset. To do so, the axioms defined in RDFS and OWL ontologies are utilised. With respect to the vocabulary terms in an RDF graph, we differentiate between the used classes and properties (i.e., the terms that are used to describe the data in an RDF graph) and the defined classes and properties (i.e., the terms whose definition is contained in the given RDF graph). For example, in Listing 5.1, dbo:Country and dbo:Place are used classes while dbo:Person and schema:Place are defined classes. The same class could be a defined class and a used class in a given RDF graph. An entity identified by an IRI is typed if at least one type declaration (rdf:type) is found in the RDG graph and labelled if at least one label ( $r d f s: l a b e l$ ) is found. For example, dbr:Sri_Lanka and dbr:Colombo are typed entities and dbr:Sri_Lanka is also a labelled entity.

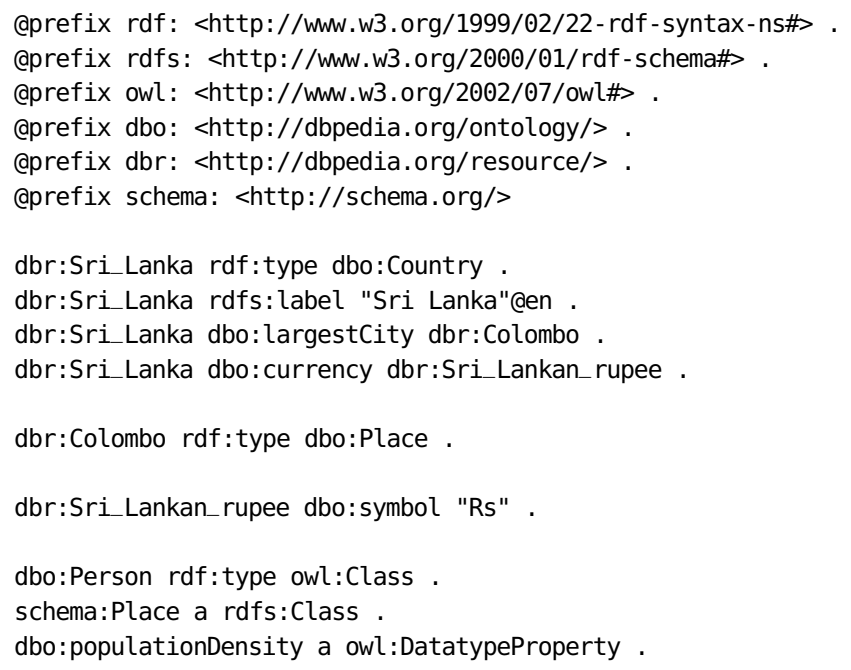

Listing 5.1: A snippet from an RDF graph

It is important to note that the vocabulary usage analysis can be performed either without any reasoning, only using the materialised triples in a given graph, or after performing reasoning. If a reasoner is used, it will derive new axioms and the profiling results will reflect values based on all these axioms. The metrics defined in this section are equally useful for both scenarios with or without reasoning but when representing the profiling results, it should be indicated if a reasoner has been used or not. 
The metrics extracted in the vocabulary usage analysis task includes the number of distinct vocabularies used in a dataset, the number of distinct classes defined in the dataset, the number of distinct classes used in the data, the number of distinct properties defined, the number of distinct properties used are extracted. Such information can be grouped into vocabulary partitions to provide aggregated information about classes and properties from a given ontology. The list of all the profiling tasks related to vocabulary usage analysis is presented in Table 5.3. For each class, the number of instances in the dataset and for each property, the number of triples containing the given property can be extracted. Such information is mainly useful for vocabulary usage analysis (U9), search and discovery (U2), and data integration (U6).

Table 5.3: Vocabulary usage analysis (T2)

\begin{tabular}{|c|c|c|}
\hline ID & Name & Description \\
\hline \multicolumn{3}{|c|}{ M2.1 - Metrics related to resources } \\
\hline M2.1.1 & typedResourceCount & $\begin{array}{l}\text { The number of resources with at least } \\
\text { one type associated with them. }\end{array}$ \\
\hline M2.1.2 & labelledResourceCount & $\begin{array}{l}\text { The number of resources with at least } \\
\text { one } r d f s: l a b e l \text { associated with them. }\end{array}$ \\
\hline \multicolumn{3}{|c|}{ M2.2 - Metrics related to classes } \\
\hline M2.2.1 & classCount & $\begin{array}{l}\text { The number of distinct classes used, e.g., } \\
\text { (?instance rdf:type ?class). }\end{array}$ \\
\hline $\mathrm{M} 2.2 .2$ & classList & The list of distinct used class IRIs. \\
\hline M2.2.3 & definedClassCount & $\begin{array}{l}\text { The number of distinct classes defined } \\
\text { within the dataset, e.g., (?class } r d f \text { :type } \\
\text { owl:Class or } r d f s: \text { Class). }\end{array}$ \\
\hline M2.11.1 & classIRI & The URI of each class. \\
\hline M2.11.2 & instanceCount & The number of instances of each class. \\
\hline M2.11.3 & instanceRatio & $\begin{array}{l}\text { The number of instances of each class di- } \\
\text { vided by the number of total resources. }\end{array}$ \\
\hline \multicolumn{3}{|c|}{ M2.3 - Metrics related to properties } \\
\hline M2.3.1 & propertyCount & $\begin{array}{l}\text { The number of distinct properties used } \\
\text { in data, e.g., (?s ?property ?o) }\end{array}$ \\
\hline M2.3.2 & propertyList & The list of distinct property IRIs used. \\
\hline M2.3.3 & objectPropertyCount & $\begin{array}{l}\text { The number of distinct object properties } \\
\text { used, e.g., (?s ?property ?o) }\end{array}$ \\
\hline M2.3.4 & datatypePropertyCount & $\begin{array}{l}\text { The number of distinct datatype prop- } \\
\text { erties used, e.g., (?s ?property ?o) }\end{array}$ \\
\hline
\end{tabular}

Table 5.3-Continued on next page 
Table 5.3 - Continued from previous page

\begin{tabular}{|l|l|l|}
\hline ID & Name & Description \\
\hline M2.3.5 & definedPropertyCount & $\begin{array}{l}\text { The number of distinct properties de- } \\
\text { fined within the dataset, e.g., (<prop- } \\
\text { erty> rdf:type } \text { rdf:Property). }\end{array}$ \\
\hline T2.3.6 & propertyIRI & The URI of the given property. \\
\hline T2.3.7 & tripleCount & $\begin{array}{l}\text { The number of triples containing the } \\
\text { given property in the dataset. }\end{array}$ \\
\hline T2.3.8 & tripleRatio & $\begin{array}{l}\text { The number of triples containing the } \\
\text { given property divided by the number } \\
\text { of total triples in the dataset. }\end{array}$ \\
\hline M2.4.1 & vocabularyCount & $\begin{array}{l}\text { The number of distinct vocabularies } \\
\text { used. }\end{array}$ \\
\hline M2.4.2 & vocabularyNamespace & The namespace of each vocabulary. \\
\hline M2.4.3 & vocabularyClassCount & $\begin{array}{l}\text { The number of classes used from each } \\
\text { vocabulary. }\end{array}$ \\
\hline M2.4.4 & vocabularyPropertyCount & $\begin{array}{l}\text { The number of properties used from } \\
\text { each vocabulary. }\end{array}$ \\
\hline
\end{tabular}

\subsubsection{Network Metrics Analysis (T3)}

In the network metrics analysis task (T3), different network metrics related to the RDF graph of the dataset are extracted. The main metrics considered in this analysis include the analysis of in-degree and out-degree. Because an RDF graph can be considered as a directed graph, for each node in the graph ( a resource in the RDF graph), its in-degree (that is, the number of triples that has the entity as the object) and its out-degree (that is, the number of triples that has the entity as the subject) can be calculated. Figure 5.2 shows an example of in-degree and out-degree metrics. Furthermore, for both in-degree values and out-degree values, a set of descriptive statistics such as min, max, mean, standard deviation can be calculated. Such metrics provide informative insights about the structure and the connectivity of the dataset.

Furthermore, other metrics considered in the network metrics analysis are related to the components in graphs. In graph theory, a component is defined as a subgraph in which all nodes are connected but the given subgraph (the component) is not connected to other subgraphs in the supergraph. Figure 5.3 shows a simplified example snippet from an RDF graph containing three components: component A, $\mathrm{B}$, and $\mathrm{C}$. They have three, five, and nine nodes, respectively. Thus, the largest component is component $\mathrm{C}$ with nine nodes. The largest component ratio is 0.53 
(i.e., nine out of 17 total nodes). The metrics related to components provides how connected are the nodes in an RDF graph.

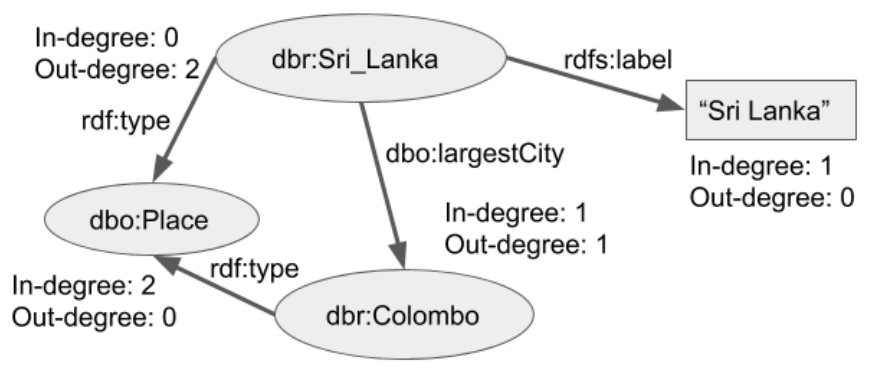

Figure 5.2: An example of in-degree and out-degree metrics

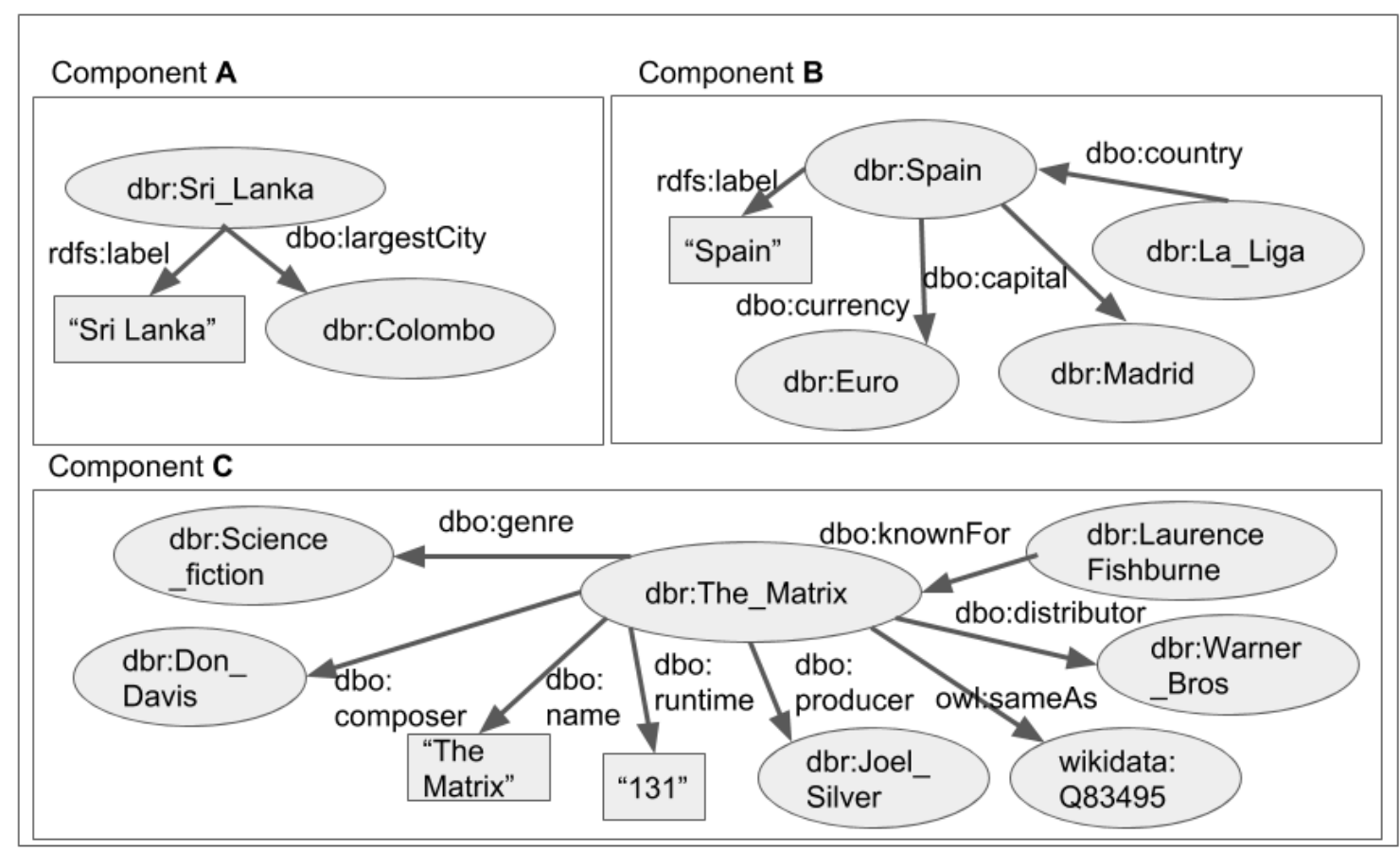

Figure 5.3: An example of 3 components in an RDF graph

Such information is useful for query optimisation (U8), quality assessment and cleansing (U5), and data integration (U6), data visualisation (U10), and data compression (U13). 
Table 5.4: Network metrics analysis (T3)

\begin{tabular}{|c|c|c|}
\hline ID & Name & Description \\
\hline \multicolumn{3}{|c|}{ M3.1 - Metrics related to in-degree } \\
\hline M3.1.1 & Mean in-degree & The average in-degree of all objects. \\
\hline M3.1.2 & Max in-degree & The maximum in-degree of all objects. \\
\hline M3.1.3 & Min in-degree & The minimum in-degree of all objects. \\
\hline M3.1.4 & $\begin{array}{l}\text { Std. deviation of in- } \\
\text { degree }\end{array}$ & $\begin{array}{l}\text { The standard deviation of in-degree of } \\
\text { all objects. }\end{array}$ \\
\hline \multicolumn{3}{|c|}{ M3.2 - Metrics related to out-degree } \\
\hline M3.2.1 & Mean out-degree & The average out-degree of all subjects. \\
\hline M3.2.2 & Max out-degree & $\begin{array}{l}\text { The maximum out-degree of all sub- } \\
\text { jects. }\end{array}$ \\
\hline M3.2.3 & Min out-degree & The minimum out-degree of all subjects \\
\hline M3.2.5 & $\begin{array}{l}\text { Std. deviation of } \\
\text { out-degree }\end{array}$ & $\begin{array}{l}\text { The standard deviation of out-degree of } \\
\text { all subjects. }\end{array}$ \\
\hline \multicolumn{3}{|c|}{ M3.3 - Metrics related to components } \\
\hline M3.3.1 & Component Count & $\begin{array}{l}\text { The number of components in the RDF } \\
\text { graph. }\end{array}$ \\
\hline M3.3.2 & $\begin{array}{l}\text { Largest Component } \\
\text { Size }\end{array}$ & $\begin{array}{l}\text { The number of nodes in the largest com- } \\
\text { ponent. }\end{array}$ \\
\hline M3.3.3 & $\begin{array}{l}\text { Largest Component } \\
\text { Ratio }\end{array}$ & $\begin{array}{l}\text { The number of nodes in the largest com- } \\
\text { ponent divided by the total number of } \\
\text { components in the RDF graph. }\end{array}$ \\
\hline
\end{tabular}

\subsubsection{Triple Pattern Analysis (T4)}

In the triple pattern analysis task (T4), triples are analysed to identify common patterns. The information extracted in this analysis includes all the properties associated with instances of a given class, abstract triple pattern counts, property sets, class sets. Abstract triple patterns are used for extracting relations between the ontology classes used in data based on the analysis of triples. For a given triple $<$ subject, property, object $>$, the corresponding abstract triple patterns are defined by <subjectType, property, objectType $>$ where subjectType is any class that is defined as the type of the subject of the triple (i.e., subject rdf:type subjectType) and objectType is any class that is defined as the type of the object of the triple (i.e., object rdf:type objectType). For each subject and object, there can be zero, one, or many types defined in the data. The owl:Thing class is not considered when extracting abstract triple patterns as it leads to trivial and non-informative patterns. If there are no types defined for either the subject or the object, no abstract triple 
patterns can be extracted. In all other cases, a set of abstract triple patterns can be extracted as illustrated in Figure 5.4.

\section{Abstract Pattern(s)}

Triple

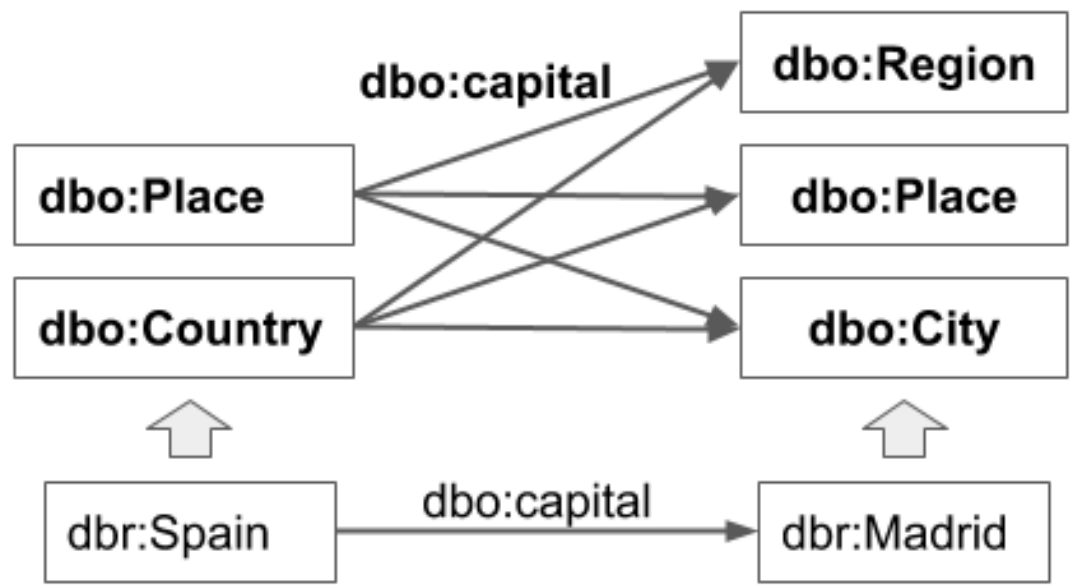

Figure 5.4: An example of abstract triple patterns

A property set is a set of properties that commonly occur together. For instance, in person entities in DBpedia, properties such as foaf:name, dbo:birthDate, dbo:birthPlace, dbo:deathDate, dbo:occupation, and dbo:nationality occur commonly together. The property set count specifies the number of instances that have a given set of properties in common. Similarly, a class set is a set of classes that commonly occur as types of a given instance. Generally, these can either be equivalent classes, superclasses, or subclasses. For example, a class set could include foaf:Person, dbo:Person, wikidata:Q5, and $d b o:$ Athlete. The class set count specifies the number of instances that are typed as all the classes in a given class set.

Furthermore, a set of new metrics are defined considering different parts of the abstract triple patterns.

Such information is useful for data-driven schema induction (U4), instance-based ontology matching (U7), search and discovery (U2), data summerisation (U3), monitoring dataset changes (U11) and data quality assessment and cleansing (U5).

Table 5.5: Triple pattern analysis (T4)

\begin{tabular}{|l|l|l|}
\hline ID & \multicolumn{1}{|c|}{ Name } & Description \\
\hline \multicolumn{2}{|c|}{ M4.1 - Metrics related to abstract triple patterns } \\
\hline M4.1.1 & subjectType & The subject type of the abstract triple pattern \\
\hline M4.1.2 & predicate & The predicate of the abstract triple pattern \\
\hline
\end{tabular}

Table 5.5 - Continued on next page 
Table 5.5-Continued from previous page

\begin{tabular}{|c|c|c|}
\hline ID & Name & Description \\
\hline M4.1.3 & objectType & The object type of the abstract triple pattern \\
\hline M4.1.4 & $\begin{array}{l}\text { abstractTriplePattern } \\
\text { Count }\end{array}$ & $\begin{array}{l}\text { The number of triples in the datatset con- } \\
\text { forming to the abstract triple pattern. }\end{array}$ \\
\hline M4.1.5 & $\begin{array}{l}\text { abstractTriplePattern Ra- } \\
\text { tio }\end{array}$ & $\begin{array}{l}\text { The number of triples in the datatset conform- } \\
\text { ing to the abstract triple pattern devided by } \\
\text { the total number of triples in the dataset. }\end{array}$ \\
\hline \multicolumn{3}{|c|}{ M4.2 - Metrics related to property sets } \\
\hline \multicolumn{3}{|c|}{ M4.2 - Metrics related to class sets } \\
\hline \multicolumn{3}{|c|}{ M4.3 - Metrics related to property cardinality } \\
\hline T4.4.1 & Property cardinality & $\begin{array}{l}\text { The cardinality of a given property with re- } \\
\text { spect to all instances linked with a given prop- } \\
\text { erty. }\end{array}$ \\
\hline T4.4.2 & $\begin{array}{l}\text { Class-property cardinal- } \\
\text { ity }\end{array}$ & $\begin{array}{l}\text { The cardinality of a given property with re- } \\
\text { spect to the instances of a given class. }\end{array}$ \\
\hline T4.1 & $\begin{array}{l}\text { Property domain parti- } \\
\text { tions }\end{array}$ & $\begin{array}{l}\text { Information about the subject class - property } \\
\text { combinations (implicit domains), for example, } \\
\text { Person->birthplace }\end{array}$ \\
\hline T4.1.1 & $\begin{array}{l}\text { Class-property triple } \\
\text { count }\end{array}$ & $\begin{array}{l}\text { The number of triples that follow a given class } \\
\text { property combination. }\end{array}$ \\
\hline T4.1.2 & $\begin{array}{l}\text { Class-property triple ra- } \\
\text { tio }\end{array}$ & $\begin{array}{l}\text { The class property triple count divided by } \\
\text { the total number of triples of given property. }\end{array}$ \\
\hline T4.2 & Property range partitions & $\begin{array}{l}\text { Information about the property - object class } \\
\text { (implicit ranges) combinations, for example, } \\
\text { birthPlace->City }\end{array}$ \\
\hline T4.2.1 & $\begin{array}{l}\text { Property-class triple } \\
\text { count }\end{array}$ & $\begin{array}{l}\text { The number of triples that follow a given } \\
\text { property - object class combination. }\end{array}$ \\
\hline T4.2.2 & Property-class triple ratio & $\begin{array}{l}\text { The property-class triple count divided by the } \\
\text { total number of triples of given property. }\end{array}$ \\
\hline $\mathrm{T} 4.4$ & Cardinality analysis & $\begin{array}{l}\text { Information about cardinalities of a given } \\
\text { property and a given class-property combina- } \\
\text { tion }\end{array}$ \\
\hline T4.5 & Property Set Analysis & Information about property set frequencies. \\
\hline T4.6 & Class Set analysis & Information about class set frequencies. \\
\hline
\end{tabular}




\subsubsection{Value Distribution Analysis (T5)}

In the value distribution analysis task (T5), the literal object values of distinct properties are analysed. The value distribution analysis can be done either at property level (e.g., considering all the values of property dbo:length irrespective of the types of instances they are associated with) or at class-property level (e.g., considering values only associated with instances of a Movie, dbo:Movie-dbo:length). For any type of value (e.g., strings, numerics or dates), an analysis of the frequency of each distinct value can be calculated which provides insights on what are the most common values and which are the least common. Further detailed analysis on value distribution depends on the type of values. For example, in the case of numeric a set of descriptive statistics can be calculated, such as the min value, max value, average, median, standard deviation, etc. In the case of string and IRIs, a similar analysis can be done by considering the character length of string literals or IRIs. These tasks can be extended for other data types such as dates. For instance, Table 5.6 illustrates the statistics about the values of a length of a movie in a dataset. As it can be seen, such statistics can give a general idea about the expected values of a property as well as possible outliers. In this case, the minus value for the movie length could be an outlier or an error in the data.

Table 5.6: dbo:Movie-dbo:length value distribution

\begin{tabular}{|l|l|}
\hline Metric & Value \\
\hline Min value & -1 mins \\
\hline Max value & $245 \mathrm{mins}$ \\
\hline Mean value & $128.3 \mathrm{mins}$ \\
\hline Median value & 118 \\
\hline Mode value & 120 \\
\hline Std. deviation & 5.7 \\
\hline
\end{tabular}

This information is useful for data quality assessment and cleansing (U5), data exploration (U1), data visualisation (U10), query optimisation (U8). In general, descriptive statistics about the values can provide valuable information for understanding the distribution of values of a given property. 
Table 5.7: Value distribution analysis (T5)

\begin{tabular}{|c|c|c|}
\hline ID & Name & Description \\
\hline \multicolumn{3}{|c|}{ M5.1 - Metrics related to numerical value analysis } \\
\hline M5.1.1 & Min value & $\begin{array}{l}\text { The minimum value of the set of numerical val- } \\
\text { ues of a given property in the dataset. }\end{array}$ \\
\hline M5.1.2 & Max value & $\begin{array}{l}\text { The maximum value of the set of numerical } \\
\text { values of a given property in the dataset. }\end{array}$ \\
\hline M5.1.3 & Mean value & $\begin{array}{l}\text { The average value of the set of numerical values } \\
\text { of a given property in the dataset. }\end{array}$ \\
\hline M5.1.4 & Median value & $\begin{array}{l}\text { The median value of the set of numerical values } \\
\text { of a given property in the dataset. }\end{array}$ \\
\hline M5.1.5 & Std. deviation & $\begin{array}{l}\text { The standard deviation of the set of numerical } \\
\text { values of a given property in the dataset. }\end{array}$ \\
\hline \multicolumn{3}{|c|}{ M5.2 - Metrics related to string analysis } \\
\hline M5.2.1 & Min string length & $\begin{array}{l}\text { The minimum length of the set of string values } \\
\text { of a given property in the dataset. }\end{array}$ \\
\hline M5.2.2 & Max string length & $\begin{array}{l}\text { The maximum length of the set of string values } \\
\text { of a given property in the dataset. }\end{array}$ \\
\hline M5.2.3 & Mean string length & $\begin{array}{l}\text { The average length of the set of string values of } \\
\text { a given property in the dataset. }\end{array}$ \\
\hline M5.2.4 & Median string length & $\begin{array}{l}\text { The median length of the set of string values of } \\
\text { a given property in the dataset. }\end{array}$ \\
\hline M5.2.5 & Mode string length & $\begin{array}{l}\text { The mode length of the set of string values of a } \\
\text { given property in the dataset. }\end{array}$ \\
\hline M5.2.6 & $\begin{array}{l}\text { String length Std. de- } \\
\text { viation }\end{array}$ & $\begin{array}{l}\text { The standard deviation of the set of string } \\
\text { lengths of a given property in the dataset. }\end{array}$ \\
\hline \multicolumn{3}{|c|}{ M5.3 - Metrics related to IRI length analysis } \\
\hline M5.3.1 & Min IRI length & $\begin{array}{l}\text { The minimum length of the set of IRIs of a given } \\
\text { property in the dataset. }\end{array}$ \\
\hline M5.3.2 & Max IRI length & $\begin{array}{l}\text { The maximum length of the set of IRIs of a } \\
\text { given property in the dataset. }\end{array}$ \\
\hline M5.3.3 & Mean IRI length & $\begin{array}{l}\text { The average length of the set of IRIs of a given } \\
\text { property in the dataset. }\end{array}$ \\
\hline M5.3.4 & Median IRI length & $\begin{array}{l}\text { The median length of the set of IRIs of a given } \\
\text { property in the dataset. }\end{array}$ \\
\hline M5.3.5 & Mode IRI length & $\begin{array}{l}\text { The mode length of the set of IRIs of a given } \\
\text { property in the dataset. }\end{array}$ \\
\hline
\end{tabular}

Table 5.7 - Continued on next page 
Table 5.7 - Continued from previous page

\begin{tabular}{|l|l|l|}
\hline ID & Name & Description \\
\hline M5.3.6 & $\begin{array}{l}\text { IRI length Std. devia- } \\
\text { tion }\end{array}$ & $\begin{array}{l}\text { The standard deviation of the set of IRIs of a } \\
\text { given property in the dataset. }\end{array}$ \\
\hline \multicolumn{2}{|c|}{ M5.4 - Metrics related to IRI namespace analysis } \\
\hline M5.4.1 & $\begin{array}{l}\text { Common namespace } \\
\text { IRI }\end{array}$ & $\begin{array}{l}\text { The common namespace IRI found in a set of } \\
\text { IRIs. }\end{array}$ \\
\hline M5.4.2 & $\begin{array}{l}\text { Common namespace } \\
\text { Count }\end{array}$ & $\begin{array}{l}\text { The number of IRIs conforming to the common } \\
\text { namespace in a set of IRIs. }\end{array}$ \\
\hline Ratio & $\begin{array}{l}\text { Common namespace } \\
\text { The number of IRIs conforming to the common } \\
\text { namespace in a set of IRIs devided by the total } \\
\text { number of IRIs in the set. }\end{array}$ \\
\hline
\end{tabular}

\subsubsection{Language Analysis (T6)}

In the language analysis task (T6), language tags of string literals are analysed to extract information about multilingual content in the dataset. Such information includes the number of language-tagged strings, the number of distinct language tags used in the dataset, the number of distinct string values per each language, the number of distinct languages per each property. For instance, Listing 5.2 illustrates an RDF graph with multilingual values for the rdfs:label property. This information is useful for data search and discovery (U2), data summarization (U3), and data integration (U6).

\footnotetext{
Qprefix wikidata: <http://www.wikidata.org/entity/> .

Qprefix rdfs: <http://www.w3.org/2000/01/rdf-schema\#> .

wikidata:Q11660 rdfs:label "artificial intelligence"@en;

rdfs: label "inteligencia artificial"@es;

rdfs: label "intelligenza artificiale"@it;

rdfs: label "Tehisintellekt"@et.
}

Listing 5.2: A multilingual snippet from an RDF graph

Table 5.8: Language analysis (T6)

\begin{tabular}{|c|c|c|}
\hline ID & Name & Description \\
\hline \multicolumn{3}{|c|}{ M6.1 - Metrics related to strings of a given language } \\
\hline M6.1.1 & Language & The identifier for a given language \\
\hline M6.1.2 & String count & $\begin{array}{l}\text { The number of strings tagged with a given lan- } \\
\text { guage }\end{array}$ \\
\hline
\end{tabular}

Table 5.8 - Continued on next page 
Table 5.8 - Continued from previous page

\begin{tabular}{|l|l|l|}
\hline M6.1.3 & Distinct string count & $\begin{array}{l}\text { The number of distinct strings tagged with a } \\
\text { given language }\end{array}$ \\
\hline M6.1.4 & String ratio & $\begin{array}{l}\text { The number of strings tagged with a given lan- } \\
\text { guage divided by the total number of strings }\end{array}$ \\
\hline
\end{tabular}

\subsection{Loupe Linked Data Profiling Model}

In this section, we present an information model for representing the metrics related to the six Linked Data profiling tasks that were discussed in the previous section. As discussed in Chapter 3, the current dataset description models are not expressive enough to capture the profiling metrics that are generated by the tasks described in Section 5.2. Thus, the main objective of the Loupe extended dataset description model [167], is to provide a conceptual model for representing fine-grained profiling metrics about RDF. It is modelled in such a way that it can represent the information extracted from each of the profiling tasks described in Section 5.2. The Loupe dataset description model extends the information in the existing RDF dataset descriptions models such as VoID and LODStats and proposes metrics that capture further fine-grained details. Inspired by the VoID vocabulary, it uses different partitions to describe specific details related to a set of related entities, for example, class partitions, property partitions, vocabulary partitions or language partitions. The core of the Loupe extended dataset description model is illustrated in Figure 5.5.

The model is implemented as an OWL ontology. The core of the Loupe model is illustrated in Figure 1 and it is described in the ontology documentation ${ }^{3}$.

\subsection{Loupe Linked Data Profiling Tools}

In this section, we present a set of Linked Data profiling tools for performing the aforementioned profiling tasks and representing the results in RDF. Loupe Linked Data profiling tools consist of (a) the Linked Data Profiling toolkit [163] for performing the profiling tasks and generating results, (b) the Loupe Web Portal for visual exploration of profiling results, (c) the Loupe REST API [169] for integration of Loupe with third-party tools, and (d) the profiling results repository for collecting profiling results of a large number of datasets that are publicly available.

\footnotetext{
${ }^{3}$ https://goo.gl/4rRL8Q
} 


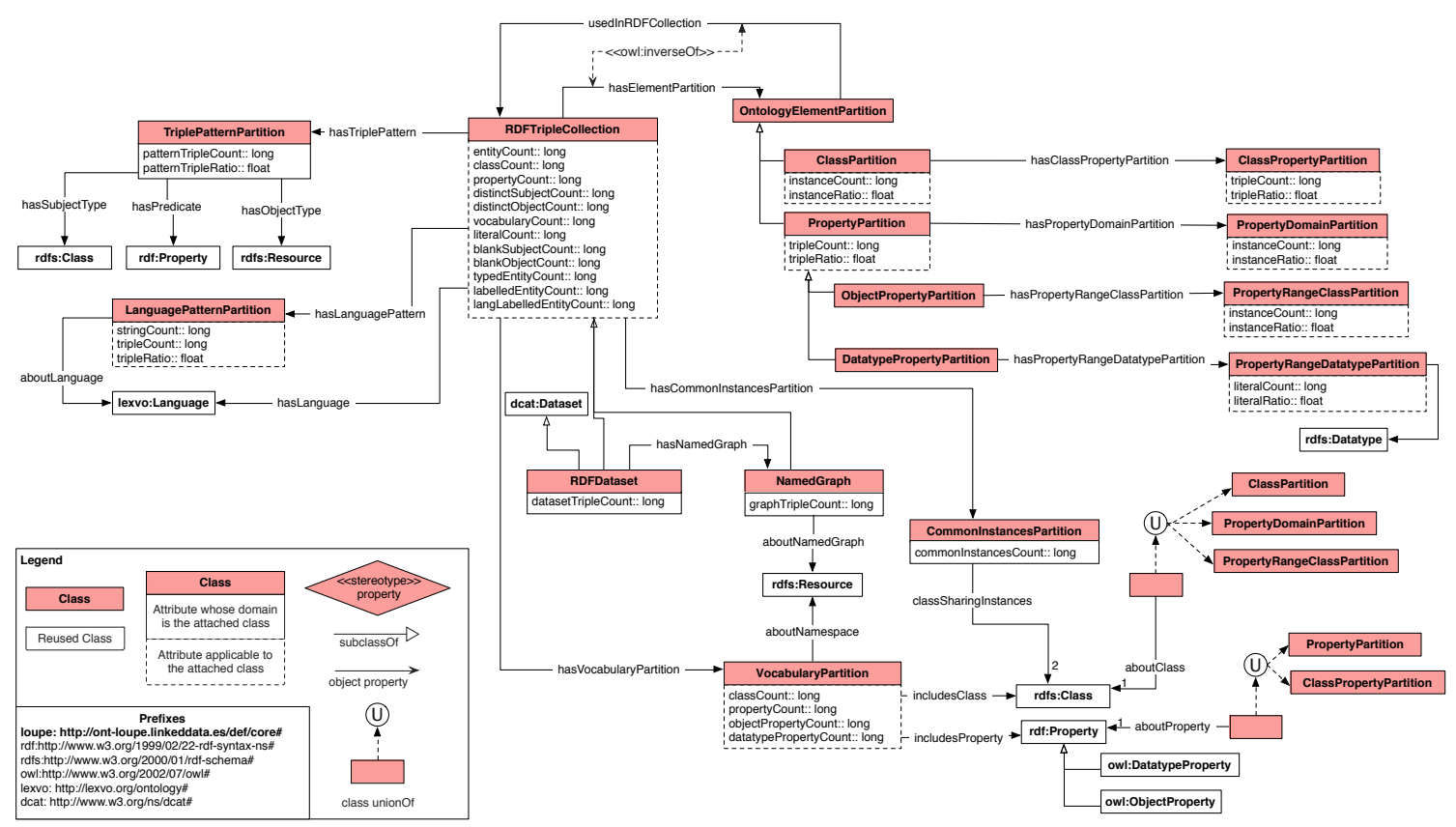

Figure 5.5: The Loupe conceptual model

\subsubsection{Linked Data Profiling Toolkit}

The Loupe profiling toolkit provides a tool suite for performing Linked Data profiling tasks. The toolkit provides the necessary infrastructure for performing the profiling tasks in a scalable manner as illustrated in Figure 5.6.

The dataset wrapper component provides the connectivity layer and the endpoints for performing SPARQL queries to perform the Linked Data profiling tasks discussed in Section 5.2 and generate the metrics for each task. It can be configured to use several different sources such as public SPARQL endpoints, Linked Data Fragments servers, or RDF dumps. In the cases of public SPARQL endpoints and Linked Data Fragment servers, the queries are executed against their query endpoint. In such cases, the responsibility of the dataset wrapper component is limited to running a set of exploratory queries to discover the potential server limits of results to adjust the limits and offsets of the profiling queries. In the case of RDF dumps, Loupe uses the Docker virtualization for creating a docker container that has a pre-installed Virtuoso triple store [68]. A bash script is to decompress the RDF dump (if necessary), generate the loading scripts for Virtuoso, and create a docker container which will expose a virtualized SPARQL endpoint to query the data in the RDF dump. The docker image used for this task is publicly available in the Dockerhub ${ }^{4}$. This approach is preferred compared to the use of public endpoints because it prevents executing

\footnotetext{
${ }^{4}$ https://hub.docker.com/r/nandana/virtuoso-oss - 7 - loupe/
} 
a large number of queries on those endpoints, which might cause low performances for other users and not comply with fair use. Furthermore, it might lead to some undesired and undeterministic profiling results due to different server-side limits and other configurations.

The Loupe Core component is designed as a Java application that carries out the profiling tasks based on the settings that are provided using a configuration file. The configuration file contains information about (a) the data source and how to access it, (b) the profiling tasks to be performed and the metrics to be generated, and (c) how the profiling results should be represented and stored. The Loupe Core component is implemented based on the popular Spring Boot ${ }^{5}$ framework for building enterprise Java applications and RDF processing is performed by reusing the Jena ${ }^{6}$ open source library. The Loupe Core component is designed following a modular architecture with each module responsible for one of the profiling tasks defined in Section 5.2. Furthermore, each metric extraction is defined as a service worker that can run parallel in its own thread so that they can be executed in isolation based on their configuration. For example, all profiling tasks related to a certain ontology class used in a dataset, is performed using a service worker that takes the class IRI as the input, performs all the queries, and fills the results. The service workers contain a set of SPARQL query templates that are parameterised so that the service worker just fills the relevant parameters and execute the queries. This design of the Loupe Core component allows the framework to select of the service workers that need to be run, configure them with correct parameters, and run them in parallel in multiple threads to get the maximum advantage of the available CPUs in current servers. The Loupe Core component detects the available resources such as the number of CPUs and the available memory, and automatically adjusts its configuration such as the number of threads in thread pools to scale according to the available infrastructure.

Once the profiling tasks are completed, the Loupe Core component generates the profiling results in one or two formats based on the configuration. On the one hand, it generates the RDF representation of the results using the Loupe extended dataset description model discussed in the previous section. This Linked Data profiling information representation in RDF makes it possible to reuse them using SPARQL queries for any of the Linked Data profiling use cases mentioned in Section 5.1. On the other hand, it generates a $J S O N$ representation of the results that is optimised for visualisations in the Loupe Web portal that is discussed in the next section. The motivation for generating this optimised representation is to provide a better response time and an enhanced user experience in the Loupe Web portal. These generated JSON representations are stored in an ElasticSearch engine ${ }^{7}$. This JSON

\footnotetext{
${ }^{5}$ https://spring.io/

${ }^{6}$ https://jena.apache.org

${ }^{7}$ https://Www.elastic.co
} 


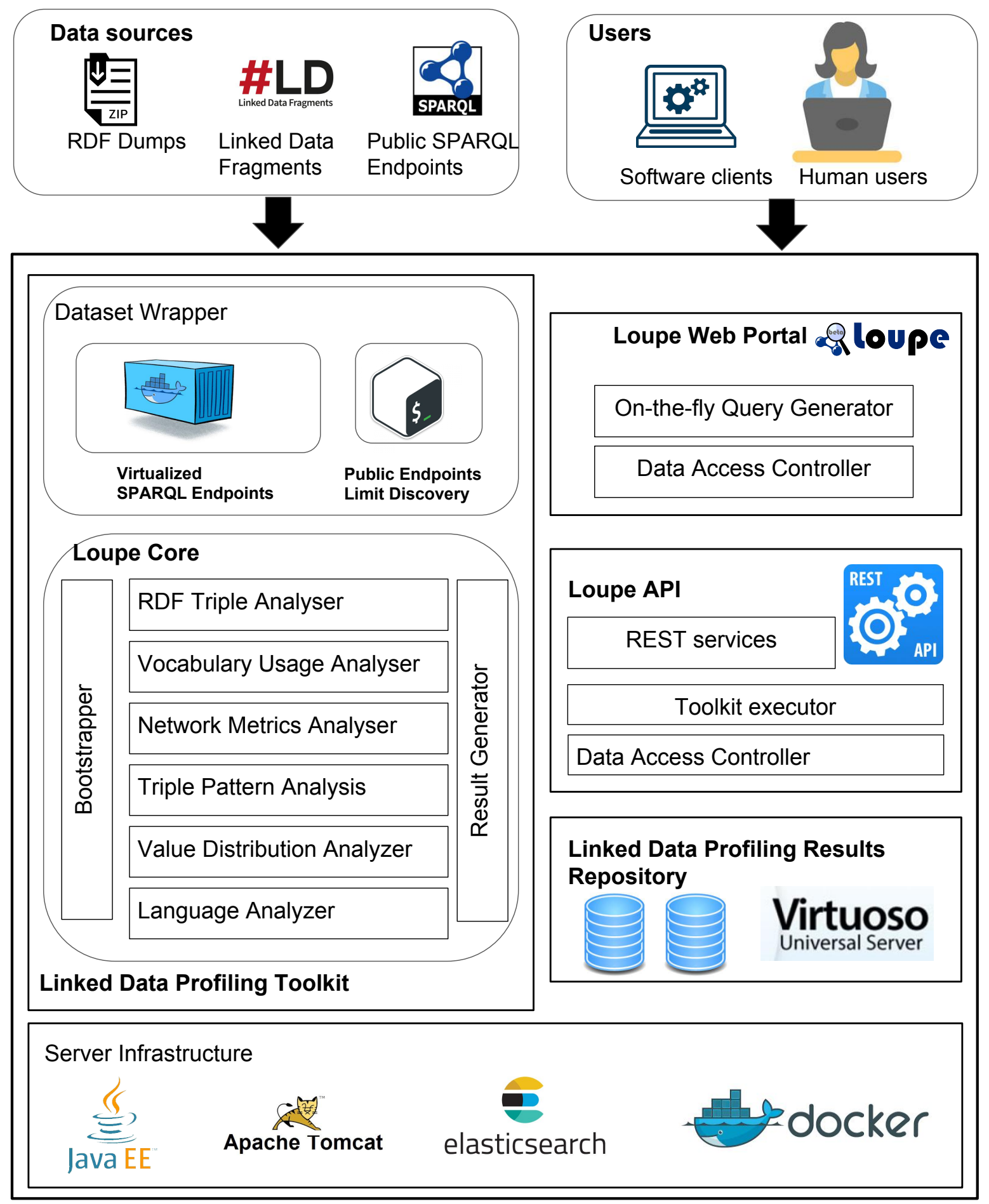

Figure 5.6: Architecture of Loupe Linked Data profiling tools 
representation is modelled aligned with the different views in the Web portal so that no complex queries are needed to generate the views and the text indexing features in ElasticSearch are better suited for tasks such as auto-completion of class or property names.

\subsubsection{Loupe Web Portal}

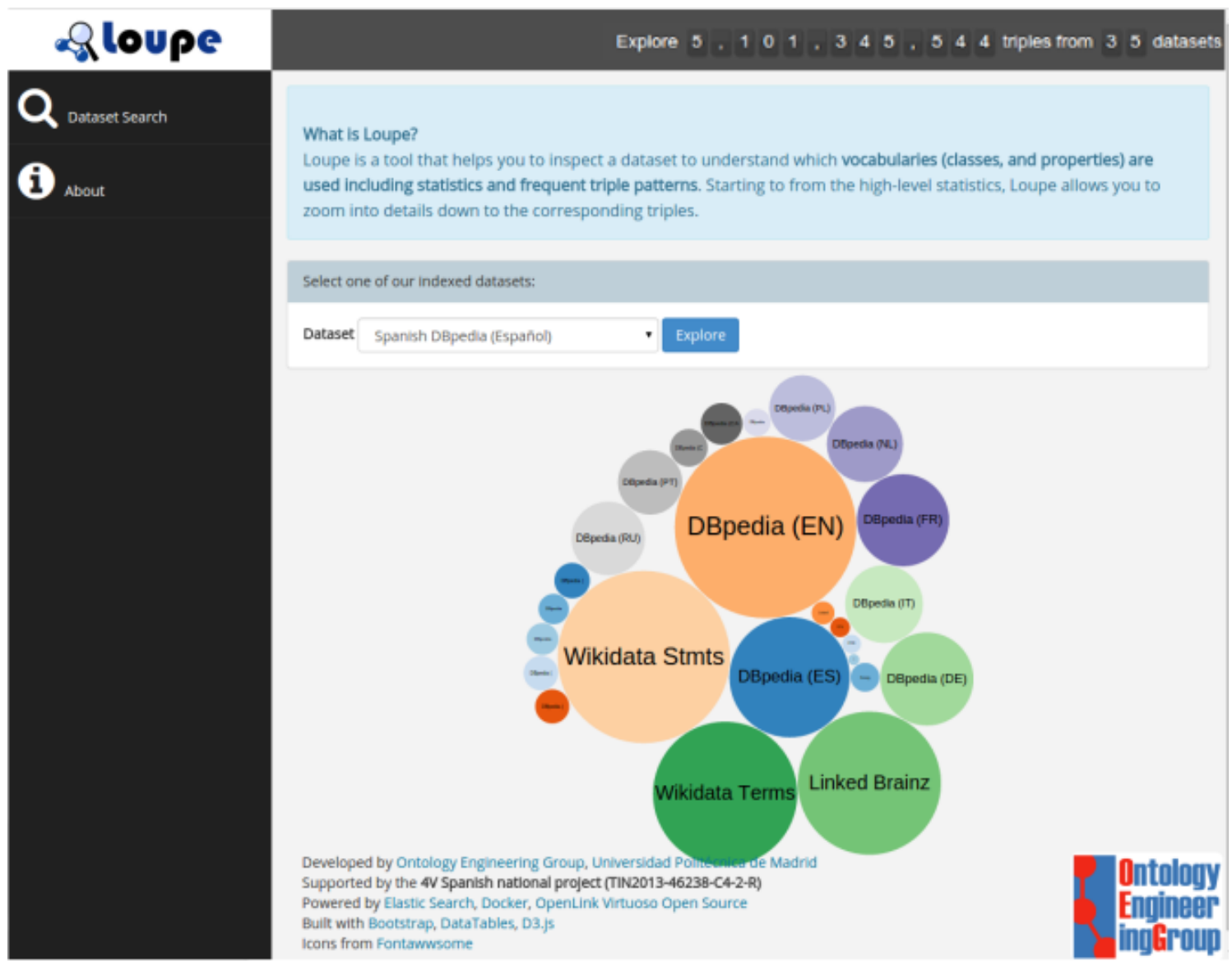

Figure 5.7: Loupe web portal

The Loupe Web Portal provides an intuitive web interface for users to inspect the datasets profiled by Loupe. The Loupe Web UI consumes the JSON representations of the Loupe profiling results generated by Loupe Core and provides several UI components that allow exploring the data extracted from the six different profiling categories: (a) RDF triple analysis, (b) vocabulary usage analysis, (c) network metrics analysis, (d) triple pattern analysis, (e) value distribution analysis, and (f) language analysis. 
Figure 5.7 illustrates a screenshot of the landing page of the Loupe Web Portal. The Loupe Web Portal can be configured to connect to an existing Elasticsearch engine which is pre-loaded with the profiling metrics about datasets using the Loupe Core component. The Web Portal connects to the ElasticSearch engine and loads the UI with the information of the datasets. From the landing page, a user can select a dataset to explore and the information about the given dataset will be available for exploration. At first, a dataset summary page is presented which mainly contains the information extracted in the RDF triple analysis. A class explorer section and a property explorer section allow a user to explore information extracted from vocabulary usage analysis in an interactive manner. All input fields are provided with auto-completion support to complete the names of known classes, properties, and namespaces in order to make the portal easy to use. Each of these UI components provides several levels of information starting from high-level overviews and then allowing users to navigate into further details. For instance, information coming from value distribution analysis, network metrics analysis, and language analysis is provided when a user has selected a certain class or a property. The triple pattern explorer section provides information extracted from triple pattern analysis.

One of the use cases of Loupe Web Portal is to allow testers to identify the different defective patterns in the data. To make this task more effective, the information provided in the Loupe Web portal is directly linked to the data when a public SPARQL endpoint for the data is available. This is done by dynamically generating an SPARQL query that will extract the data that corresponds to a given statistic. For example, when an abstract triple pattern is presented it will also have a link that will extract all the triple that comply with that pattern from the data.

\subsubsection{Loupe REST API}

The Loupe $\mathrm{API}^{8}$ is a configurable Linked Data profiling service. This RESTful interface facilitates the integration of the Loupe profiling services to other systems. Similar to the Loupe Profiling Toolkit, the Loupe API may be configured to specify how to access the source data, the profiling tasks to be performed, and how the profiling results should be represented.

The Loupe API is implemented using Java and Spring Boot RESTful services and currently three operations are available as illustrated in Figure 5.8. The documentation is provided as a Swagger interface which also acts as a client to test the services directly using the web browser.

\footnotetext{
${ }^{8}$ http://api. loupe. linkeddata.es/
} 


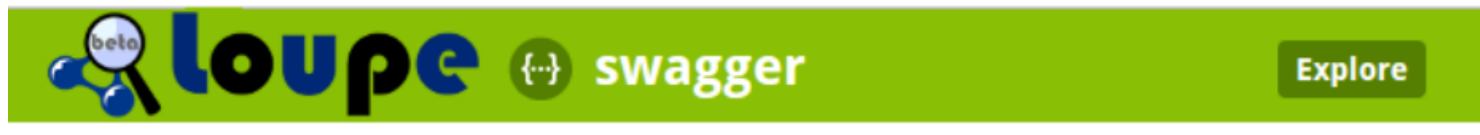

\section{Loupe Linked Data Profiler API}

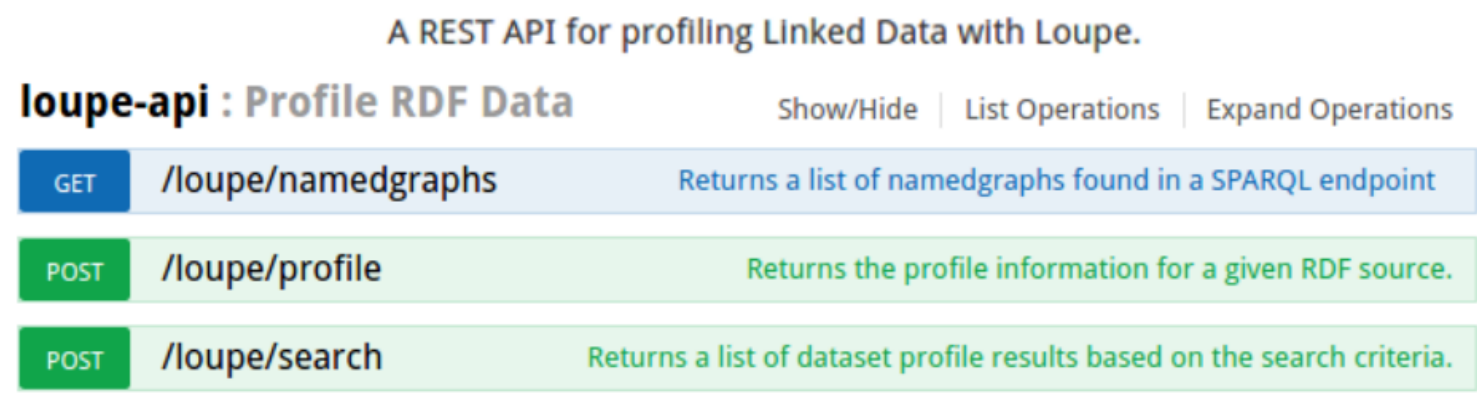

[ BASE URL: http://api.loupe.linkeddata.es/ , API VERSION: 1.0.0]

Figure 5.8: Loupe API documentation

\subsubsection{Profiling Results Repository}

This section presents the Linked Data profiling results repository. The goal of the profiling repository is to provide a collection of profiling metrics about the existing datasets in the Linked Data Cloud. The information in the data profiling repository can be used for the use cases that were mentioned in Section 5.1.

The Linked Data profile repository has used LOD Laundromat [24] as its main data source. LOD Laundromat includes datasets collected by catalog-specific scripts (e.g., the Datahub ones) and provides a representative set of datasets from the Linked Open Data cloud [201].

In addition, several datasets were included in the Loupe profiling results repository based on the user requests such as the Spanish Library data (BNE) and 3Cixty Knowledge Base. Table 5.9 shows a summary of the datasets included in the Loupe Linked Data profiling repository.

It is important to note that the Loupe profiling results repository contains a considerably large number of datasets than the ones that appear in the Linked Data Cloud diagram ${ }^{9}$. For example, by July 2018, the LOD Cloud diagram contained 1,220 datasets, whereas the Loupe profiling results repository contained 26,063 datasets. This is because the LOD Cloud diagram is restrictive in its inclusion policy.

Table 5.10 shows the most widely used vocabularies in the datasets in the Loupe Linked Data profiling results repository.

\footnotetext{
${ }^{9}$ https://lod-cloud.net
} 
Table 5.9: Overview of Loupe profiling results repository

\begin{tabular}{|l|r|}
\hline Description & Value \\
\hline Number of datasets & 26,063 \\
\hline Number of resources & $3,316,552,778$ \\
\hline Number of triples & $34,556,443,529$ \\
\hline Distinct vocabularies & 7,198 \\
\hline Number of distinct classes & 606,847 \\
\hline Number of distinct properties & $1,037,478$ \\
\hline
\end{tabular}

As expected, the list contains the vocabularies RDF, RDFS, and OWL as most datasets use the classes and properties from those vocabularies to describe the types and other schema-related information. Interestingly, there was a namespace with a typo (http://xmls.com/foaf/0.1/ instead of http://xmlns.com/foaf/0.1/) that was used by 5,418 datasets.

Table 5.11 shows the most widely used classes in the datasets in the Loupe profiling results repository. The majority of them are related to the statistical datasets because they contain a large number of instances. For instance, the one with the most instances is db:Observation.

Table 5.10: Most widely used vocabularies in datasets

\begin{tabular}{|l|r|}
\hline Vocabulary & \# of datasets \\
\hline http://www.w3.org/1999/02/22-rdf-syntax-ns\# & $18,074(69.35 \%)$ \\
\hline http://www.w3.org/2000/01/rdf-schema\# & $18,036(69.20 \%)$ \\
\hline http://purl.org/dc/terms/ & $16,131(61.89 \%)$ \\
\hline http://xmlns.com/foaf/0.1/ & $10,516(40.34 \%)$ \\
\hline http://www.w3.org/2002/07/owl & $8,574(32.90 \%)$ \\
\hline http://purl.org/linked-data/cube & $7,711(29.59 \%)$ \\
\hline http://purl.org/linked-data/sdmx/2009/ & $7,661(29.34 \%)$ \\
\hline http://rdfs.org/ns/void & $6,838(26.24 \%)$ \\
\hline http://www.w3.org/2004/02/skos/core & $6,283(24.10 \%)$ \\
\hline http://creativecommons.org/ns & $5,487(21.05 \%)$ \\
\hline http://xmls.com/foaf/0.1/ & $5,418(20.79 \%)$ \\
\hline
\end{tabular}


Table 5.11: Most common instances of classes

\begin{tabular}{|l|r|r|}
\hline Class & \# of instances & \# of datasets \\
\hline $\begin{array}{l}\text { http://purl.org/linked- } \\
\text { data/cube\#Observation }\end{array}$ & $1,302 \mathrm{M}(39.28 \%)$ & $7687(29.49 \%)$ \\
\hline $\begin{array}{l}\text { http://data-gov.tw.rpi.edu/2009/data-gov- } \\
\text { twc.rdf\#DataEntry }\end{array}$ & $300 \mathrm{M}(9.07 \%)$ & $491(1.88 \%)$ \\
\hline http://geovocab.org/geometry\#Geometry & $181 \mathrm{M}(5.47 \%)$ & $42(0.16 \%)$ \\
\hline $\begin{array}{l}\text { http://knoesis.wright.edu/ssw/ont/sensor- } \\
\text { observation.owl-\#MeasureData }\end{array}$ & $144 \mathrm{M}(4.34 \%)$ & $6(0.02 \%)$ \\
\hline http://www.w3.org/ns/dcat\#Distribution & $84 \mathrm{M}(2.53 \%)$ & $5363(20.58 \%)$ \\
\hline http://www.wikidata.org/ontology\#Statement & $57 \mathrm{M}(1.72 \%)$ & $2(0.00 \%)$ \\
\hline http://www.wikidata.org/ontology\#Item & $51 \mathrm{M}(1.56 \%)$ & $2(0.00 \%)$ \\
\hline http://purl.org/vocab/frbr/core\#Item & $51 \mathrm{M}(1.55 \%)$ & $9(0.03 \%)$ \\
\hline $\begin{array}{l}\text { http://knoesis.wright.edu/ssw/ont/- } \\
\text { weather.owl\#TemperatureObservation }\end{array}$ & $43 \mathrm{M}(1.30 \%)$ & $6(0.02 \%)$ \\
\hline http://www.wikidata.org/ontology\#Article & $42 \mathrm{M}(1.27 \%)$ & $1(0.00 \%)$ \\
\hline
\end{tabular}

Table 5.12 shows the most widely used properties in the datasets in the Loupe profiling results repository. As expected, the most widely used property in the profiled datasets is rdf:type. The second most common property is qb:dataset and this is because of the large number of statistical datasets present in the repository. Because the repository contains a large number of instances from different versions and language editions of DBpedia and each of those resource contains links to Wikipedia, the property dbo:wikiPageWikiLink is frequent in data. In general, properties related to statistics vocabularies are dominant in the most widely used properties because there are a large number of instances related to statistical observations.

Table 5.12: Most commonly used properties

\begin{tabular}{|l|r|r|}
\hline Property IRI & \# of triples & \# of datasets \\
\hline $\begin{array}{l}\text { http://www.w3.org/1999/02/22-rdf-syntax- } \\
\text { ns\#type }\end{array}$ & $3,316 \mathrm{M}(9.59 \%)$ & $18,119(69.52 \%)$ \\
\hline http://purl.org/linked-data/cube\#dataSet & $1,302 \mathrm{M}(3.76 \%)$ & $7,679(29.46 \%)$ \\
\hline $\begin{array}{l}\text { http://dbpedia.org/ontology/- } \\
\text { wikiPageWikiLink }\end{array}$ & $1,289 \mathrm{M}(3.73 \%)$ & $91(00.35 \%)$ \\
\hline $\begin{array}{l}\text { http://purl.org/linked- } \\
\text { data/sdmx/2009/dimension\#timePeriod }\end{array}$ & $1,243 \mathrm{M}(3.59 \%)$ & $7,598(29.15 \%)$ \\
\hline
\end{tabular}

Table 5.12 - Continued on next page 
Table 5.12 - Continued from previous page

\begin{tabular}{|l|r|r|}
\hline Property IRI & \# of triples & \# of datasets \\
\hline $\begin{array}{l}\text { http://purl.org/linked- } \\
\text { data/sdmx/2009/dimension\#freq }\end{array}$ & $1,243 \mathrm{M}(3.59 \%)$ & $7,596(\%)$ \\
\hline $\begin{array}{l}\text { http://purl.org/linked- } \\
\text { data/sdmx/2009/measure\#obsValue }\end{array}$ & $1,236 \mathrm{M}(3.57 \%)$ & $7,661(29.14 \%)$ \\
\hline http://www.w3.org/2002/07/owl\#sameAs & $1,021 \mathrm{M}(2.95 \%)$ & $7,030(26.97 \%)$ \\
\hline $\begin{array}{l}\text { http://eurostat.linked- } \\
\text { statistics.org/property\#geo }\end{array}$ & $886 \mathrm{M}(2.57 \%)$ & $5,115(19.62 \%)$ \\
\hline $\begin{array}{l}\text { http://eurostat.linked- } \\
\text { statistics.org/property\#unit }\end{array}$ & $540 \mathrm{M}(1.56 \%)$ & $3,072(11.79 \%)$ \\
\hline $\begin{array}{l}\text { http://www.w3.org/2000/01/rdf- } \\
\text { schema\#label }\end{array}$ & $496 \mathrm{M} \mathrm{(1.43 \% )}$ & $11,530(44.24 \%)$ \\
\hline
\end{tabular}

\subsection{Future Work}

In this study, we have identified a comprehensive set of profiling tasks which can generate useful metrics for quality assessment and repair use cases. Thus, we were focused mostly on the schema-based profiling. A similar exercise can be done for topic-based profiling and linkage-based profiling to define similar sets fine-grained of profiling tasks useful for other use cases that were identified in Table 5.1. Furthermore, in this study our classification of tasks were based on the type of analysis performed. Nevertheless, it would be interesting to perform a similar classification based on different use cases. For example, further research can be performed to identify to create specific task profiles for each of the use cases mentioned in the chapter. Such profiles will help to decide which tasks are most relevant to a given use case and develop tools to preform only those tasks.

In the following chapters of thesis, we will identify and highlight the profiling tasks that are most relevant to Linked Data quality assessment and repair tasks. 


\section{Chapter 6}

\section{RDF Shape Induction}

As discussed in Chapter 3, the third objective of this thesis is to develop an automatic RDF Shape Induction method to generate constraints using a datadriven approach without requiring significant manual effort, using the data profiling information discussed in Chapter 5. This chapter presents the RDF Shape Induction method using Linked Data profiling metrics as illustrated by Figure 6.1.

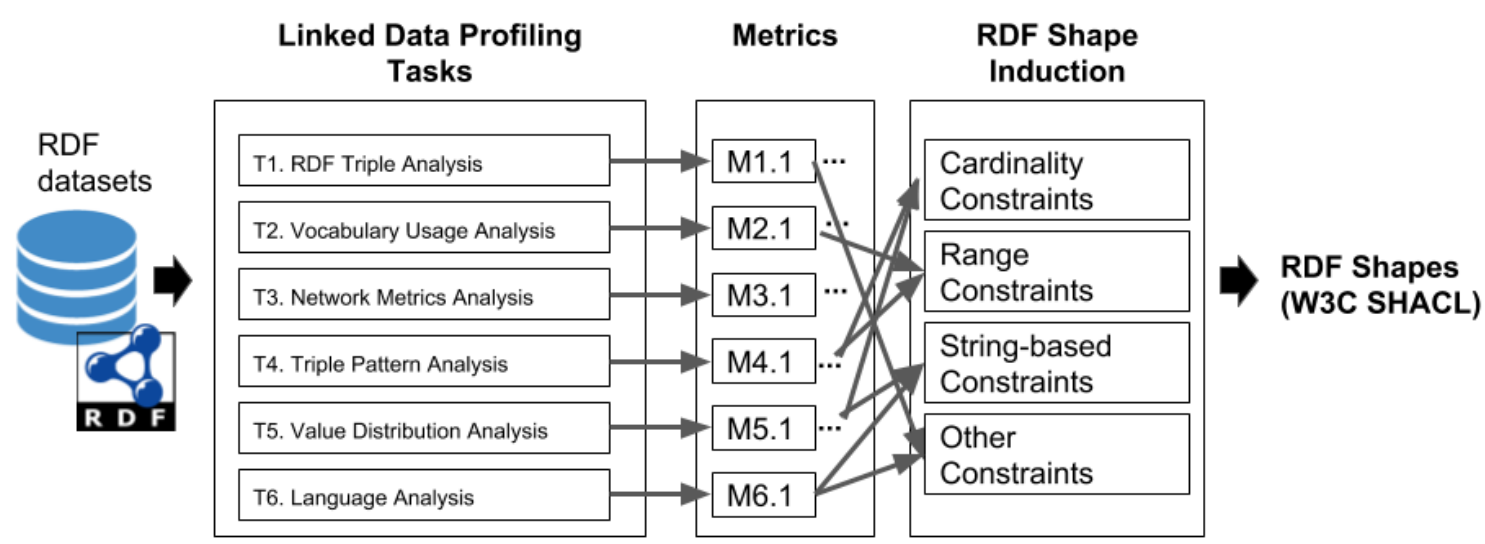

Figure 6.1: RDF Shape induction using Linked Data profiling metrics

Large-scale datasets, such as, DBpedia [17] or Wikidata [227], contain millions of facts about general knowledge facts while datasets such as Bio2RDF [27] or MusicBrainz [217] contain facts about a specific domain, in this case bio-science and music. As it was discussed in Chapter 4, for these datasets to become useful for a given use case, they should have a required level of quality or should be "fit for intended use in operations and decision-making" [129]. To ensure that a dataset has a certain level of quality, a set of validations have to be preformed. When datasets becomes large and are generated using fairly uncontrolled processes such as 
crowd-sourcing, the data validation becomes a tedious task.

As with any other data model, in order to validate that the data represented in an RDF graph satisfy a certain level of quality or fit for the intended use, a set of conditions have to to be specified based on the requirements of the use case. Among the different quality characteristics discussed in the Linked Data Quality Model, in this chapter we are focused on the consistency, i.e., the degree to which data have attributes that are free from contradiction and are coherent with other data in a specific context of use [114]. Requirements regarding the consistency of data are generally specified using the integrity constraints [198]. In practical settings, integrity constraints are commonly used for three main tasks: (a) specifying properties of the data should hold; (b) handle contradictions within the data or with respect to the domain under consideration; or (c) as a help for query optimisation. In this thesis, we are mainly interested in the tasks (a) and (b).

RDF has proven to be a good model for data integration due to its flexibility (i.e., triples from different graphs can be easily fused together irrespective of the vocabularies used to describe them and the entities can be easily to linked to each other using the global identifiers). There are a number of applications using RDF either for data storage or as an interoperability layer [193]. However, this flexibility brings inherited challenges to the quality assessment and validation of data represented using the RDF model. The lack of explicit schema in RDF data is main reason behind most of these challenges [180]. As we discuss later, the ontologies such as RDF Schema (RDFS) [43] and Web Ontology Language (OWL) [109] are designed to be computational logic-based languages that allow to perform reasoning and infer new knowledge rather than to be a schema-language that is designed for validation.

In most mainstream deployments in the industry, relational databases still remain more popular. One key reason for this is that these technologies offer better validation tools to define and exchange data conforming to a specific schema. In contrast, in RDF graphs the schema is rather implicit and changes based on the data in the graph and the different ontologies that are used to describe data. Assessing the quality of data represented using the RDF data model is a non-trivial task, as it often means measuring the quality of structured information, ontologies and vocabularies, and queryable endpoints.

Nevertheless, Semantic Web related approaches such as the RDF model are more suited for knowledge representation and reasoning. The most prominent expressive knowledge representation and reasoning methods are based on Description Logic[20]. In particular, OWL is based on Description Logic and it is a recommended standard by the World Wide Web Consortium (W3C) for modelling ontologies. Description Logics (DLs), in turn, bear a first-order predicate logic semantics. DLs are monotonic and adhere to the Open World Assumption (OWA). This means that (negative) conclusions drawn from a knowledge base (e.g., which could be represented as an RDF graph) 
must be based on information explicitly present in the knowledge base. On the other hand, under Closed World Assumption (CWA) all non-provable expressions are assumed to be false. OWA makes it challenging to perform certain validation tasks [210]. For example, a minimum cardinality constraint are not generally violated under OWA because there is always a possibility that a triple exists somewhere which will fulfill the cardinality requirement.

The main reason for ontological languages to be not suitable for the describing validation constraints is the fact that they are designed for inferring new knowledge using known axioms rather than for validating data based on the axioms. A reasoner and a validator have different functions, i.e., a reasoner is used for inferring new knowledge while a validator is used finding violations against a set of constraints. The underpinning principles used in OWL, such as the use of Open World Assumption (OWA) and Non-Unique Name Assumption, can lead to unexpected results in a validator [210].

Most traditional data modelling paradigms such as relational databases follow a process commonly known as schema on write [64]. In such cases, a comprehensive schema with the conditions and constraints that should be held are defined before loading any data and validation process is performed for every data write to ensure that the write operation does not change integrity of the stored data. In contrast, most RDF triple stores and applications follow a more flexible model that is known as schema on read $[64,151]$. In this approach, generally only syntactic level validations are preformed during data writes, and the schema of the data evolves based on the data inserted. Even-though this makes it possible insert data with diverse schema into RDF stores, this dynamic nature of the schema makes it harder have a concrete set of validation constraints for a given dataset. Thus, for RDF Data it is valuable to have approaches that can automatically discover the schema of the data at any given time.

A dataset is defined to be consistent if it does not contain conflicting or contradictory data [110]. When a schema is available with the integrity constraints that the data should comply, the data usually goes through a validation process that verifies the compliance against those constraints. These integrity constraints encapsulate the consistency requirements for the data in order to be fit to a given set of use cases. For instance, in the relational databases, the integrity constraints are expressed in a data definition language (DDL), and the database management systems (DBMS) ensure that any data inserted into the database will not lead to any inconsistency in the entire database. However, validation of RDF data is not done in this manner due to several reasons such as the lack of a language for expressing constraints (RDFS and OWL are designed for entailment and not for validation as discussed in the introduction), or having generic models suitable for wider use and not for specific use cases. Furthermore, generally RDF data from different sources are commonly put 
together making such validations on insertions less feasible.

Validation against a pre-defined schema to ensure that a given dataset is consistent is one of the key steps in most conventional data storage and publishing paradigms, for instance, validation against Data Description Language (DDL) constraints in SQL databases or XML Schema or RelaxNG in XML documents [193]. For RDF data, typically OWL or RDF Schema based ontologies are used for doing this kind of validations. When an ontology is available with TBox axioms that define the conceptualisation of the domain, a reasoner can be used to verify whether the dataset is consistent with the domain by verifying the axioms defined in the ontology. However, most of the ontologies do not have rich axioms that could help to detect inconsistencies in data. Most of the large knowledge bases, such as DBpedia, lack a definition of integrity constraints and it is a tedious task to manually create these constraint definitions from scratch. Furthermore, most of the schema information about RDF data is only available in the form of OWL ontologies that are most suited for entailment rather than validation. Though this is the case, most practical use cases that utilise RDF data need the data to be validated for integrity constraints. The use of ontologies for validating RDF data leads to several problems: (a) the mismatch between intended purposes, inference (for which the ontologies are designed for) and validation, and (b) lack of information useful for validation in most of the ontologies. Thus, it is necessary to represent the constraints for validating data in a more suitable language.

Our objective is to utilise the profiling information discussed in the previous chapter to address the challenge of automatically inducting RDF Shapes and then to represent them in a language suitable for representing constraints for data validation. To this end, we propose a data driven approach by applying machine learning techniques on profiled RDF data to automatically generate integrity constraints. Then all constraints related a given class are combined to generate an RDF Shape, that is, a collection of constraints. The proposed approach is defined in a generic way that applies to any type of constraints. Aligned with the main objective of this thesis, the goal of generating RDF Shapes from data is to use them in quality assessment and repair techniques.

\subsection{RDF Shapes}

In recent years, there is an increase of using read and write Linked Data for use cases such as Enterprise Application Integration [162]. For instance, IBM has been using the Linked Data principles for the OSLC standard and has been using OSLC Resource Shapes [209] for performing "schema on write" like operations with RDF data. In the OSLC specification, a resource shape is defined as a resource $A$ that

describes the content of another RDF resource $B$ and the constraints that has to be 
satisfied by the resource $B$. The shape resource $A$ is also represented as RDF using the OSLC vocabulary.

The OSLC Resource Shapes specification was submitted to W3C in 2014 as a starting point to create a new W3C recommendation. Since then, the W3C community has put an effort to standardise a language for describing structural integrity constraints and validation rules for RDF instance data within the RDF Data Shapes Working Group ${ }^{1}$ and later on within the Shape Expression Community Group $^{2}$. The outcomes of such efforts, languages such as W3C Shapes Constraint Language (SHACL) [137] and Shape Expressions Language [194], allow integrity constraints to be defined for RDF data and validate data.

When describing the constraints for validating RDF data, a language should be able to describe several types of restrictions. For example, (a) RDF terms restrictions such as allowed RDF node types (IRI, blank nodes, literals), allowed values (value ranges, datatypes, patterns), (b) cardinality restrictions such as maximum, minimum, and exact cardinalities, (c) predicate constraints such as required predicates. Both SHACL and ShEx have the expressivity for expressing such constraints [221]. In this we have chosen to represent the RDF Shapes in SHACL because it is already a W3C Recommendation that has a comprehensive process of validation by the W3C community before the publication. Nevertheless, the proposed approach is on dependent on the constraint representation language and it could be easily amended for representing the generated shapes in another constraint language such as ShEx.

Shapes Constraint Language (SHACL) is a language for validating RDF graphs against a set of conditions. SHACL provides the necessary language constructs for expressing the constraints as RDF Shapes. In SHACL, there are two main RDF graphs involved: (a) shapes graph which contains the shape expressions for validating certain data, (b) data graph which contains the actual data to be validated against the constraints in the shapes graph. In the RDF Shape Induction method proposed in this thesis, we follow a data-driven approach to start from the data graph and to generate the shape graph using automatic induction of RDF shapes.

The SHACL specification consists of two sub-specifications: SHACL Core and SHACL-SPARQL. SHACL Core contains the common features necessary for representing frequently used shapes and constraints. SHACL-SPARQL contains of all features of SHACL Core plus the advanced features of SPARQL-based constraints and an extension mechanism to declare new constraints. In this work, we only focus on the SHACL Core specification.

A SHACL validator takes as an input a data graph and a shapes graph (both represented in RDF) and generate a validation report that shows which nodes of the data graph have conformance to the shapes graph. The SHACL Core specification

\footnotetext{
${ }^{1}$ https://www.w3.org/2014/data-shapes/charter

${ }^{2}$ https://www.w3.org/community/shex/
} 
defines three main types of components: (a) targets; (b) property paths; and (c) conditions.

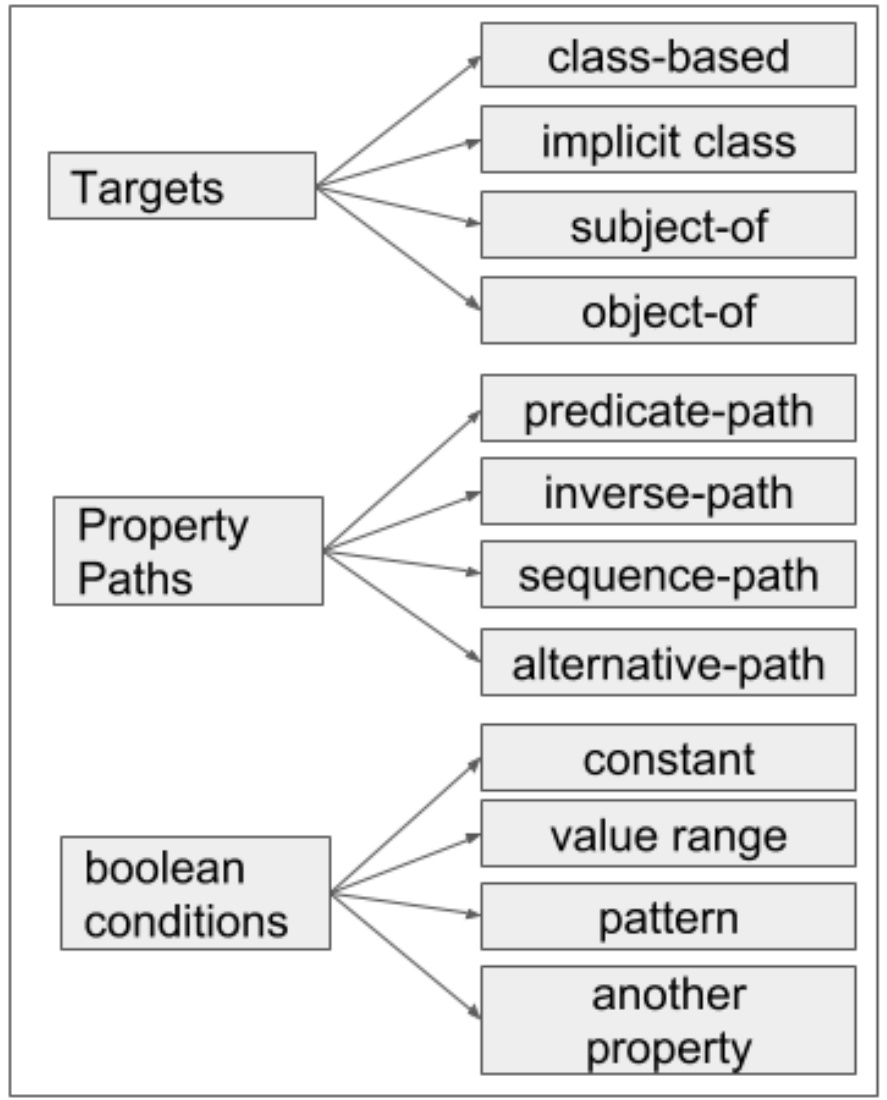

Figure 6.2: SHACL-Core components

Targets allow the shapes to define a set of nodes of the data graph that the validation should be carried out, known as focus nodes. Targets can be either node targets (concrete nodes in the data graph), class-based and implicit class targets (all nodes of a certain class in the data graph), or subject-of and object-of targets (all nodes which are subject or object of a given property). Because the RDF Shape Induction approach proposed in this thesis is based on learning the constraints by identifying patterns in data, it is more suitable for class-based, implicit class, subject-of and object-of targets.

Property paths defines how to reach the property value nodes from the focus nodes. For this, SHACL uses a subset of SPARQL Property Paths [105] which includes paths such as PredicatePath, InversePath, SequencePath, AlternativePath [137]. Predicate paths are property IRIs and in this work, we are focused on generating constraints with predicate paths. 
Conditions define how a SHACL validator check for conformance of a given value and validate it. They are boolean predicates that compare a property value with a constant, a value range, a pattern or another property value that is reachable from the same focus node. In this work, we focus on constant, value range and pattern condition. Table 6.1 illustrates a set of conditions that can be expressed using SHACL.

Listing 6.1 shows a snippet of a shapes graph with an example RDF Shape related person information. The target of this shape is defined using the "sh:targetClass" property which has a value of "dbo:Person". As discussed before, this defines focus nodes, i.e., which nodes in the data graph is validated with this shape. In this case, all the instances of class "dbo:Person" will become focus nodes. For example, if we consider the data graph shown in Listing6.2 this includes the nodes _ :1 and _:2 but not dbr:Gampaha. The shape contains several predicate paths, i.e., foaf:name, dbo:birthDate, and dbo:birthPlace properties. For the values that are reaches using each of these properties, there are some conditions that they should satisfy. For example, there should be exactly one value for the dbo:birthDate and it should be a literal of type $x s d$ :date.

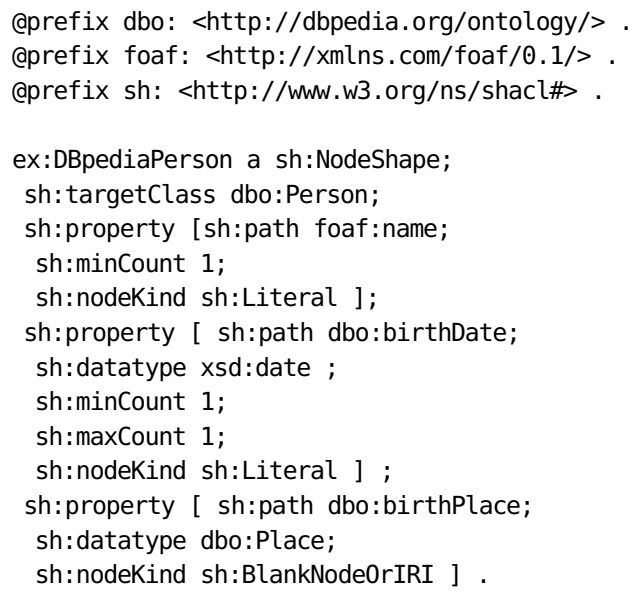

Listing 6.1: A snippet from a shapes graph

If we pass the shape graph shown in Listing 6.1 and the data graph shown in Listing6.2 to a SHACL validator, it will generate a validation report with all the violations of the constraints in data.

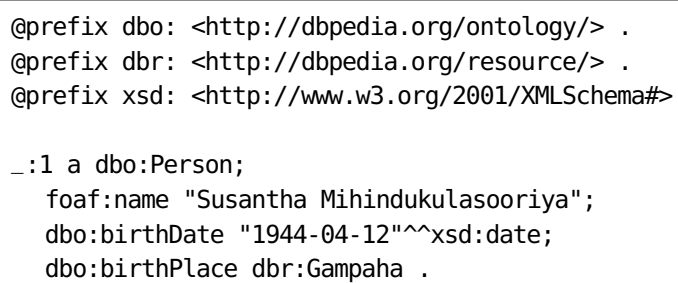




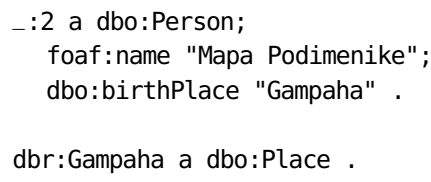

Listing 6.2: A data graph snippet

Table 6.1: Constraints in SHACL

\begin{tabular}{|l|l|}
\hline Constraints Type & Example \\
\hline Cardinality & minCount, maxCount \\
\hline Types of values & class, datatype, nodeKind \\
\hline Values & node, in, hasValue \\
\hline Range of values & minInclusive, maxInclusive, minExclusive, maxExclusive \\
\hline String based & $\begin{array}{l}\text { minLength, maxLength, pattern, languagesIn, uniqueLan- } \\
\text { guage }\end{array}$ \\
\hline Logical constraints & not, and, or, xone \\
\hline Closed shapes & closed, ignoredProperties \\
\hline Property pair constraints & equals, disjoint, lessThan, lessThanOrEquals \\
\hline Other constraints & name, value, defaultValue \\
\hline
\end{tabular}

Generally SHACL constraints and RDF Shapes has to be generated by domain experts. In order to generate such constraints, the domain experts have to analyse the data and formulate the corresponding checks and validation rules to ensure conformance with the characteristics of the entities that data represent and other validation requirements. Nevertheless, formulating SHACL constraints manually is a tedious task and it requires a domain experts with sufficient understanding of the domain. Depending the volume of data in a dataset and the complexity of the schema, it might take a large amount of time for an expert to author these constraints. For instance, for a large RDF knowledge base such as DBpedia, which contains more than 750 classes from the DBO ontology, around 350,000 classes from the YAGO ontology and 60,000 properties (both $d b o$ : and $d b p$ :), it is a challenge to generate SHACL constraints manually.

\subsection{Generic Shape Induction Approach}

In this section, we present a data-driven approach for automatically generating SHACL constraints by using the information extracted from the Linked Data profiling metrics 
discussed in Chapter 5 in order to learn the constraints. As discussed previously, RDF Shapes can be used to validate existing RDF graphs; nevertheless, it is a tedious task to generate such RDF Shapes manually. Thus, to address the challenge of automatically generating RDF Shapes, we propose a generic workflow for automatic RDF Shape induction using information extracted from data profiling as the input. We define the workflow in a generic manner so that it can be applied to any type of constraints. This approach is motivated by the hypothesis that by analysing the frequent patterns visible in data, we could extract the implicit schema of data and represent it explicitly using a constraint language such as SHACL. Our intuition is that it is possible to generate representative RDF Shapes for a given dataset with such information. Our approach is to use machine learning algorithms to learn constraints from data.

Analysing patterns in data to extract useful information has been a research topic of interest in many fields related to different data modes. To this end, a large number of special purpose systems and techniques have been developed for solving such constraint-based mining and learning problems [55]. Constraints can be used both in a descriptive setting, such as discovering association rules [7], and in a predictive setting, such as rule learning [88]. Association rule mining is about discovering interesting relations between different variables in large databases and it was introduced in [6] with an example of relationships between items that are purchased in a single transaction by a customer in a supermarket. In such setting, association rule mining can uncover interesting and non-obvious relations such as male customers buying diapers usually buy beer as well [241]. In RDF data, association rules are generally used to discover relationships between distinct RDF properties (i.e., the predicates of triples in an RDF graph). For example, in a given RDF graph, association rules can discover that an entity has properties foaf:name, dbo:birthPlace, and dbo:occupation and it highly probable that it also has dbo:birthDate property. Association rule mining has been applied to RDF datasets for various tasks including generating property axioms [79], predicate suggestions, data enrichment, ontology improvement, query relaxation [1, 2, 179], and semantic search engines [218]. In this work, the integrity constraints that we aim to generate are not limited to relationships between two properties but also include relationships between a property and a value. For example, the range of a certain property or a cardinality of a certain property with respect to a given class. Thus, in this study we explore other machine learning algorithms for learning constraints.

The key idea of our approach is to map each constraint generation problem to a machine learning problem. To this end, we propose to identify and use the most appropriate machine learning algorithm for a selected constraint generation problem. Generally, machine learning algorithms can be of three main types: (a) supervised learning, (b) unsupervised learning, and (c) reinforcement learning [216]. 
Furthermore, there are other variations such as semi-supervised learning, transductive inference, on-line learning, or active learning [173].

In supervised learning, an algorithm learns the relation between a certain output based on a set of predefined inputs (commonly known as features) by analysing a set of example input-output pairs (commonly known as training data). Typical machine learning tasks that are solved by supervised machine learning algorithms include classification and regression. In classification problem, the algorithms must learn to assign a category (or a label) to a given new instance based on the categories of instances the algorithm as already seen. The number of categories can be either two (binary classification) or more (multi-label classification). In regression, the goal is to generate an output given a set of inputs. Nevertheless, in contrast to classification algorithms, the outputs of regression can have a large range of continuous numerical values such as floating point numbers or integers instead of a small discrete categories.

In unsupervised learning, unlike in supervised learning, labelled training data is not required. Clustering is one of the typical unsupervised machine learning tasks in which a set of instances are divided into groups based on a set of input features. These groups are not known in advance and no training data is required. Association learning is also a task for unsupervised machine learning. Reinforcement learning algorithms are goal-oriented and used in tasks such as playing a game or driving a car in which the algorithm interacts with a dynamic environment and learns based on the positive and negative rewards. In the proposed approach for RDF Shape induction, for each constraint generation problem we explore the different machine learning algorithms to identify the best way to formulate the constraint generation problem as a classical machine learning problem as discussed above.

In this thesis, we provide examples for three types of constraints, namely, (a) cardinality constraints, (b) range constraints, and (c) string-based constraints. Following the same steps as in those three, the proposed approach can be extended to other types of constraints such as value range constraints (min, max values), property pair constraints (lessThan, lessThanOrEquals, disjoint, equal) [137].

Furthermore, instead of learning constraints on the raw data, in our approach we use data profiling information as the input features to the machine learning algorithms. The main reason for using profiling information instead of raw data is to increase the efficiency so that same profiled data can be used for many constraint generation problems. In following sections, we discuss how profiled data can be applied to RDF shape induction, an area that has not been addressed for RDF data validation problems in the current literature.

As discussed before, our main hypothesis is that machine learning techniques can be used to automatically generate correct RDF Shapes by using data profiling information as features. In the coming sections, we define an approach for generating RDF Shapes and validate our hypothesis by evaluating the generated RDF Shapes 
to ensure that the constraints that were generated are accurate by using a manually labelled gold standard (See Section 8.3). The goal of the proposed approach is to extract the constraints by analysing data patterns and statistics and combine the extracted constraints to generate RDF Shapes that can be used for validation. The main steps of the proposed workflow are illustrated in Figure 6.3. The input to the workflow is statistical profiling information coming from the Loupe Linked Data Profiling Framework as discussed in Section 5.2.

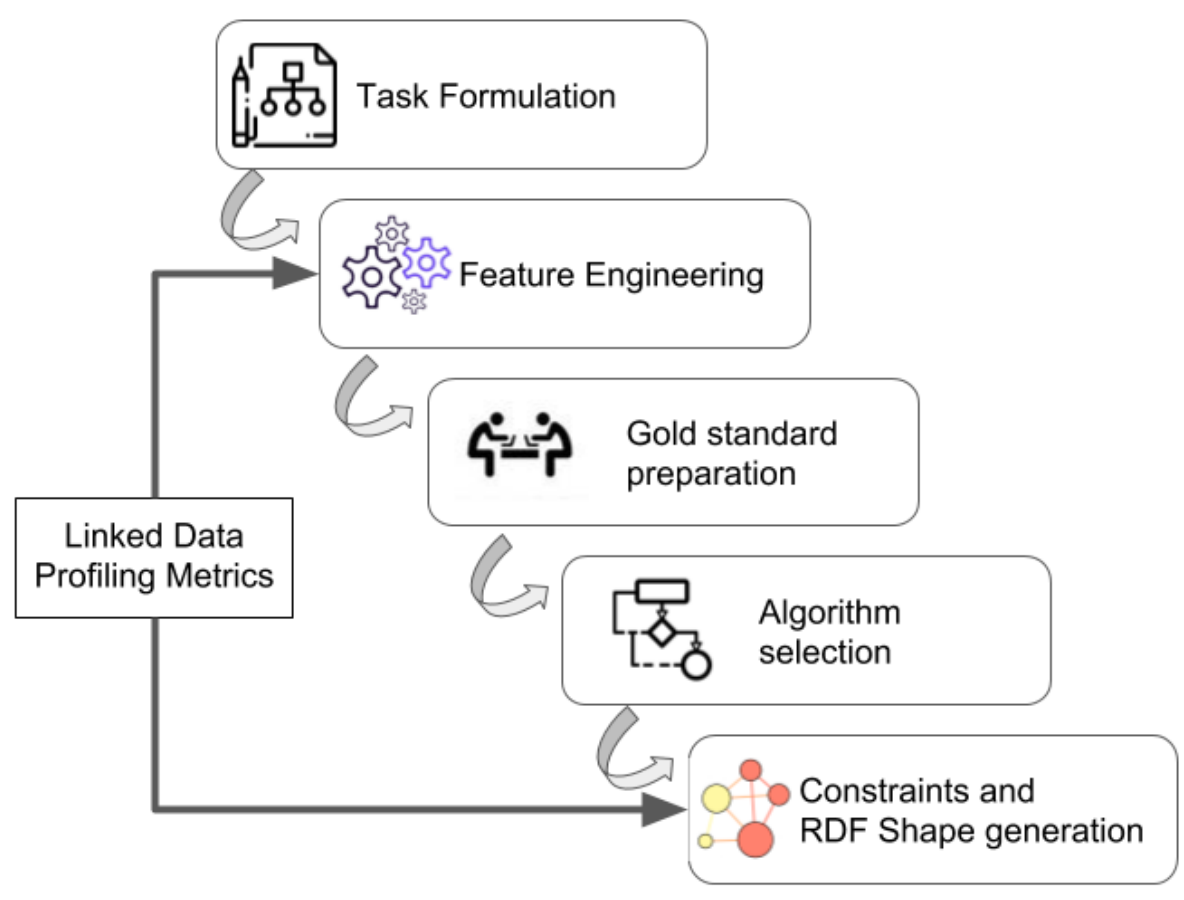

Figure 6.3: A method for RDF Shape induction.

\subsubsection{Task Formulation}

The first step of the workflow is the formulation of the specific constraint extraction goal as a typical machine learning task. The type of the machine learning algorithm is mainly influenced by the type of the expected output. For example, if the output is a few discrete values such as a set of categories, the task can be formulated as a classification problem. If the output is a continuous numerical value, the problem can be formulated as a regression task. Similarly, for each task, depending on its expected output, the most appropriate machine learning task can be selected. Nevertheless, other factors such as common patterns in data can have an influence in the task formulation as we discuss in the next section (e.g., using categories for cardinalities instead of absolute values). For instance, the range of the output could be open to 
large number of values in theory while in practice it could be limited to a set of predefined values. To this end, it is necessary to understand the requirements and the characteristics in real world scenarios where a given constraint is applicable and use that as an input for the task formulation step.

In the following sections, we will illustrate a running example of task formulation for three types of constraints: (a) cardinality constraints, (b) range constraints, and (c) string-based constraints.

Cardinality constraints: Generally, cardinality constraints are expressed using two constraints: (a) minimum cardinality and (b) maximum cardinality. Thus, our goal is to generate two cardinality constraints (whenever it is relevant). Because the output of the minimum cardinality or the maximum cardinality constraints could be any real value, at a first glance it seems a good candidate for a regression task.

Nevertheless, as discussed before, it is important to know the characteristics of the constraints in the real world datasets. Thus, as the first step of understanding the task better, we manually annotated the expected cardinalities (i.e., a gold standard) for a subset of 215 properties from the 3cixty knowledge base, 166 properties from the Spanish National Library (BNE) dataset, and 175 properties from DBpedia. After analysing cardinalities of those 556 properties in all three datasets, we found that all those can be categorised into a few common patterns. Table 6.2 shows the common cardinality patterns we found in the gold standard.

Table 6.2: Minimum and maximum cardinality levels

\begin{tabular}{|l|l|}
\hline Key & Description \\
\hline MIN0 & Minimum Cardinality $=0$ \\
\hline MIN1 & Minimum Cardinality $=1$ \\
\hline MIN1+ & Minimum Cardinality $>1$ \\
\hline MAX1 & Maximum Cardinality $=1$ \\
\hline MAX1+ & Maximum Cardinality $>1$ \\
\hline
\end{tabular}

In order to further validate if that patterns apply commonly for other datasets and not only in the datasets we considered, we expanded our study to check the explicit cardinality constraints defined in public vocabularies that are available in the Linked Open Vocabularies (LOV) catalogue. We could observe the same trend in the vocabularies where the cardinality constraints were explicitly defined. When we analysed the 551 vocabularies in LOV for the cardinality constraints values, $96.91 \%$ (848 out of 875) of owl:maxCardinality constraints have value 1 and $93.76 \%$ (631 out of 673 ) of the owl:minCardinality values either 0 or 1.

Thus, in our work rather than trying to estimate the exact numbers for the minimum cardinality and maximum cardinality values, we decided to formulate the cardinality constraint generation task as two classification problems. The task 
of generating the minimum cardinality constraint of a given property (when it is associated with an instance of a given class) is formulated as assigning it to one of the following groups: MIN0, MIN1, or MIN1+ (See Table 6.2). Similarly, the task of generating the maximum cardinality for a property is formulated as assigning it as one of the following groups: MAX1, or MAX1+. By doing so, we could reduce the problem from a regression problem to a classification problem.

Range constraints: Regarding ranges of properties, the type of constraints that could be generated are twofold. The first type of constraint is the object node kind. According to the RDF data model [52], a property value (i.e., the object of a triple) can be either an IRI, a blank node, or a literal [52]. Thus, the object node kind can be constrained to one of those types or their combinations. For this work, we have selected the node kinds already identified in SHACL, i.e., IRI, Literal, BlankNode, BlankNodeOrIRI, BlankNodeOrLiteral, and IRIOrLiteral. Table 6.4 shows some example for object node kind constraints.

Table 6.3: Object node kind examples

\begin{tabular}{|l|l|}
\hline Class-property & $\begin{array}{l}\text { Expected } \\
\text { kind }\end{array}$ \\
\hline dbo:Person-dbo:birthPlace & IRI \\
\hline dbo:Place-dbo:geo & BlankNodeOrIRI \\
\hline dbo:Person-dbo:name & Literal \\
\hline dbo:Person-dbo:deathDate & Literal \\
\hline dbo:Person-dbo:religion & IRIOrLiteral \\
\hline
\end{tabular}

The second type of range constraint that can be generated is the type of the value of the property. This constraints is dependent on the object node type. If the node kind is Literal, the values can be constrained to a certain data type. If the node kind is either IRI, BlankNode, or BlankNodeOrIRI, the type of the entity can be restricted to an ontology class (e.g., an OWL class or an RDFS class). For the cases where the object node kind is BlankNodeOrLiteral or IRIOrLiteral, single value type constraint can not be specified; nevertheless, a combined constraint can be specified. Table 6.4 shows some example value type constraints.

Table 6.4: Value type examples

\begin{tabular}{|l|l|}
\hline Class-property & Expected datatype \\
\hline dbo:Person-dbo:birthPlace & dbo:Place \\
\hline
\end{tabular}




\begin{tabular}{|l|l|}
\hline dbo:Place-dbo:geo & geo:SpatialThing \\
\hline dbo:Person-dbo:name & xsd:string \\
\hline dbo:Person-dbo:deathDate & xsd:date \\
\hline dbo:Person-dbo:religion & dbo:Place OR sd:string \\
\hline
\end{tabular}

The first task of assigning one of the node kind constraints to each of the classproperty combination fits well as a classification problem where the different expected node kinds are the labels or categories. The second task of assigning the corresponding datatype or class as the range of each property is done based on heuristics of datatype or class type distributions among the set of objects associated with the property.

String-based constraints: There are several constraints that can be generated for string values in a dataset. These constraints include minimum string length, maximum string length, allowed languages, and unique value per language (i.e., a property should not have multiple values for a single language).

The minimum and maximum string lengths depend on the type of data expected as values. For example, for values that have to follow a certain pattern, such as the ISBN code of a book, there is a fixed length for the strings. In this case, both the minimum and the maximum value is 13 as ISBN codes are expected to have exactly 13 characters because of the predefined format. Similarly, if empty strings are not allowed, there will be a constraint of minimum string length that is one or larger. For some properties, for example in the rdfs:comment property, the string values may be restricted to one or more languages, such as English or Spanish. Table 6.5 shows some string-based constraint examples.

Table 6.5: String-based constraint examples

\begin{tabular}{|l|l|l|}
\hline Class-property & Constraint & Value \\
\hline schema:Person-foaf:name & minLength & 1 \\
\hline schema:Language-dbo:iso6393Code & maxLength & 3 \\
\hline schema:Book-rdfs:label & languageIn & en \\
\hline
\end{tabular}

For predicting the minimum and maximum string length values, we need to formulate a task that would work well with different types of values such as names, codes, comments, etc. Furthermore, we need to assume that some datasets contain errors and outliers. Thus, we follow a two step approach for the string-based constraints. First, we used the string length of the distribution to generate a set of 
initial estimates for the minimum and maximum. For minimum string length, we use the 1st percentile, 2nd percentile, 5th percentile as initial estimates. We performed an initial exploratory study which confirmed these values can capture the minimum string length in all the cases that were discussed before. With this setting, we reduce the minimum string length prediction problem to a classification problem that selects one of those values (1st percentile, 2nd percentile, 5th percentile) according to a set of inputs that provides information about the string length distribution. Similarly, for the the maximum string length we have defined the 90th, 95th, and 99th percentiles as the labels for the classification problem for predicting maximum string length. Furthermore, we have added another label to denote that a maximum string length is not applicable for the given property.

\subsubsection{Feature Engineering}

Once the constraint generation task is formulated as a machine learning problem, the next important aspect is to decide what should be used as the input to the algorithms. In machine learning, this process is called feature engineering. A feature is an attribute that is present in all the elements on which the machine learning algorithm is applied. Some attributes are more useful than others and influence more in deciding the output of the machine learning algorithm. When the most appropriate features that represent the problem are selected, they can increase the accuracy of the machine learning models. In the approach proposed in this thesis, the features are selected from the Linked Data profiling information that was discussed in Chapter 5.

Cardinality constraints: The value distribution analysis task of the Linked Data profiling extracts information about cardinality of values of each property associated with instances of a given class. For example, by analysing 1,767,272 dbo:Sport instances in DBpedia, the Loupe Linked Data profiling framework extracts the cardinality distribution for the dbo:Sport-dbo:union combination as illustrated in Table 6.6.

Table 6.6: Cardinality counts for dbo:Sport-dbo:union class-property.

\begin{tabular}{|l|l|l|}
\hline Cardinality & Instances & Frequency \\
\hline 0 & 1,662 & $84.88 \%$ \\
\hline 1 & 279 & $14.14 \%$ \\
\hline 2 & 10 & $0.05 \%$ \\
\hline 3 & 5 & $0.02 \%$ \\
\hline 4 & 2 & $0.01 \%$ \\
\hline
\end{tabular}

The raw cardinality information can provide some insights about the expected cardinality of a property. However, during the feature engineering step, this raw 
profiling data can be used to derive a set of features that can be used for predicting the cardinality.

Based on the raw values we compute the distinct cardinality value distributions similar to the one reported in Table 6.6. Note that there are three distributions, one is the raw cardinalities $(0,1,0,3,1,2,1,0,1, \ldots)$, then distinct cardinalities $(0,1,2,3,4)$ and finally one is the percentages of instances per each cardinality $(84.88 \%, 14.14 \%, 0.05 \%$, $0.02 \%, 0.01 \%)$. Further, for each of the three distributions we derive several descriptive statistical metrics including min-max cardinalities, mean, mode, standard deviation, variance, quadratic mean, skewness, percentiles, and kurtosis[84]. Our motivation for using these statistical values is that each of these could provide some insights related to different possible cardinality distributions. Thus, we use all these derived features as inputs for the machine learning algorithms for generating cardinality constraints. As future work, we plan to further analyse the relative importance of each of these metrics and reduce them to a minimal set of important features that most influence the cardinality prediction.

Table 6.7, Table 6.8, and Table 6.9 report 30 features (P1 to P30) selected for a classifier that predicts the cardinality category with example values for the $d b o$ :Sport class and dbo:union property. Table 6.7 that contains features P1 to P13 are related to raw cardinality distribution. For example, P1 present a minimum cardinality value of 0 for dbo:Sport/dbo:union, P2 presents maximum that is 4, and P4 shows that the model common cardinality value is 0 . Similarly, Table 6.8 that features P14 to P20 are related to the distinct cardinality distribution. For example, P14 shows that there were 5 distinct cardinalities and P15 shows their mean was 2. Finally, Table 6.9 that contains features P21 to P30 are related to the percentages distribution. Our intuition is that these are descriptive to classify the cardinality category. Nevertheless, the data can be noisy and either min or/and max could be outliers. To address this we add statistical features that give more insights about the distribution of the cardinalities such as mean, mode, kurtosis, standard deviation, skewness, variance and four percentiles.

Table 6.7: dbo:Sport-dbo:union P1 to P13 statistical measures from the raw cardinality distribution

\begin{tabular}{|l|l|l|}
\hline ID & Description & Example \\
\hline P1 & Min Cardinality & 0 \\
\hline P2 & Max Cardinality & 4 \\
\hline P3 & Mean & 0.16445 \\
\hline P4 & Mode & 0 \\
\hline P5 & Quadratic mean & 0.44972 \\
\hline P6 & Kurtosis & 13.7897 \\
\hline \multicolumn{2}{|l|}{ Table 6.7 - Continued on next page }
\end{tabular}


Table 6.7 - Continued from previous page

\begin{tabular}{|l|l|l|}
\hline P7 & Standard Deviation & 0.41868 \\
\hline P8 & Skewness & 3.09484 \\
\hline P9 & Variance & 0.17529 \\
\hline P10 & 98th percentile & 1 \\
\hline P11 & 2nd percentile & 0 \\
\hline P12 & 75nd percentile & 0 \\
\hline P13 & 25th percentile & 0 \\
\hline
\end{tabular}

Table 6.8: dbo:Sport-dbo:union P14 to P20 statistical measures from distinct cardinality values distribution

\begin{tabular}{|l|l|l|}
\hline ID & Description & Example \\
\hline P14 & Distinct Cardinalities & 5 \\
\hline P15 & Distinct Mean Card. & 2 \\
\hline P16 & Distinct Quadratic Mean & 2.4495 \\
\hline P17 & Distinct Kurtosis & -1.2 \\
\hline P18 & Distinct Standard Deviation & 1.5811 \\
\hline P19 & Distinct Skewness & 0 \\
\hline P20 & Distinct variance & 2.5 \\
\hline
\end{tabular}

Table 6.9: dbo:Sport-dbo:union P20 to P30 statistical measures from cardinality percentage distribution

\begin{tabular}{|l|l|l|}
\hline ID & Description & Example \\
\hline P21 & Percentages of min value & 0.0010 \\
\hline P22 & Percentage of max value & 0.8488 \\
\hline P23 & Cardinality 0 Percentage & 0.8488 \\
\hline P24 & Cardinality 1 Percentage & 0.1429 \\
\hline P25 & Percentage Mean & 0.2 \\
\hline P26 & Percentage Quad. Mean & 0.3849 \\
\hline P27 & Percentage Kurtosis & 0.3849 \\
\hline P28 & Percentage Standard Deviation & 0.3677 \\
\hline P29 & Percentage Skewness & 2.0948 \\
\hline P30 & Percentage Variance & 0.1352 \\
\hline
\end{tabular}

Range constraints: The RDF triple analysis task (T1) and the vocabulary usage analysis task (T2) of Linked Data profiling discussed in Chapter 5 provide 
insightful statistics about the number of IRIs, literals, and blank nodes for each property associated with instances of a given class as shown in Table 6.10. For both IRIs and literals, both absolute object counts (counting each object multiple times when they appear in multiple triples) as well as the distinct object counts can be used as input features for predicting the range constraints. Furthermore, their percentages can also be used for gain further insights. In addition to the IRIs and Literals, the blank node counts are also calculated by the Linked Data profiling framework but it is not shown in Table 6.10 as there were no blank nodes in this concrete example, i.e., the blank nodes counts were all zero. All this information is used as features for the machine learning algorithms for classifying each class-property combination into one of the node kinds: IRI, Literal, BlankNode, BlankNodeOrIRI, BlankNodeOrLiteral, and IRIOrLiteral.

Table 6.10: Object node type information

\begin{tabular}{|l|l|l|l|l|l|l|l|l|}
\hline \multirow{2}{*}{ Class-property } & \multicolumn{4}{|c|}{ IRI } & \multicolumn{4}{c|}{ Literals } \\
\cline { 2 - 9 } & Total & \% & Distin & $\%$ & Total & $\%$ & Distin. \% \\
\hline $\begin{array}{l}\text { dbo:Person- } \\
\text { dbp:birthPlace }\end{array}$ & 89,355 & 66.69 & 21,845 & 51.70 & 44,639 & 33.31 & 20,405 & 48.30 \\
\hline $\begin{array}{l}\text { dbo:Person- } \\
\text { dbp:name }\end{array}$ & 21,496 & 15.65 & 15,746 & 13.50 & 115,848 & 84.35 & 100,931 & 86.50 \\
\hline $\begin{array}{l}\text { dbo:Person- } \\
\text { dbp:deathDate }\end{array}$ & 127 & 0.19 & 111 & 0.34 & 65,272 & 99.81 & 32,449 & 99.66 \\
\hline $\begin{array}{l}\text { dbo:Person- } \\
\text { dbp:religion }\end{array}$ & 8,374 & 54.55 & 786 & 65.88 & 6,977 & 45.45 & 407 & 34.12 \\
\hline
\end{tabular}

Furthermore, the vocabulary usage analysis task (T2) and the triple pattern analysis task (T4) in Chapter 5 extract the object type information by analysing all the IRI and blank node objects of triples containing each property as the predicate. Table 6.11 shows an example of object type information by analysing all the objects of the dbo:Person-dbp:deathPlace class-property combination. It contains the number of objects, the number of distinct of objects of each class type and their respective percentages. The same information is illustrated as a histogram in Figure 6.4. As it can be seen, the objects of dbo:Person-dbp:deathPlace were typed as many different classes. As it can be seen most objects are typed with multiple classes (e.g., with equivalent classes, super classes). Also there are some objects that should not be associated (i.e., inconsistent) with the dbp:deathPlace property, for example, a dbo:Broadcaster should not be a death place of a person. Furthermore, there are some objects for which the type information is not available. We extract all the type information available and use that as input features for classifying the object class type of a class-property combination. 
Table 6.11: Type of dbo:Person-dbp:birthPlace objects

\begin{tabular}{|c|c|c|c|c|c|}
\hline \multirow[t]{2}{*}{ Class ID } & \multirow[t]{2}{*}{ Object Class } & \multicolumn{2}{|c|}{$\begin{array}{c}\text { Objects } \\
(838,474)\end{array}$} & \multicolumn{2}{|c|}{$\begin{array}{c}\text { Distinct Objects } \\
(64,371)\end{array}$} \\
\hline & & Count & $\%$ & Count & $\%$ \\
\hline 1 & schema:Place & 678,521 & 80.92 & 59,705 & 92.75 \\
\hline 2 & dbo:Place & 677,884 & 80.84 & 57,301 & 89.01 \\
\hline 3 & dbo:PopulatedPlace & 676,607 & 80.69 & 56,400 & 87.62 \\
\hline \multicolumn{6}{|c|}{ other rows are omitted for brevity } \\
\hline 13 & dbo:Village & 34,514 & 04.11 & 9,473 & 14.72 \\
\hline 14 & dbo:Town & 28,669 & 03.42 & 8,231 & 12.79 \\
\hline \multicolumn{6}{|c|}{ other rows are omitted for brevity } \\
\hline 273 & schema:Restaurant & 1 & 00.00 & 1 & 00.00 \\
\hline 274 & dbo:Reptile & 1 & 00.00 & 1 & 00.00 \\
\hline & Unknown & 97,890 & 11.67 & 5,888 & 00.00 \\
\hline
\end{tabular}

Similarly, for literal objects, the value distribution analysis task (T5) in Chapter 5 extracts the information about their data types. Table 6.12 shows an example of extracted information for the class-property combination dbp:Person-dbp:deathDate. For each datatype, it shows the number of objects, number of distinct objects, and their corresponding percentages. This information provides heuristics about which should be the corresponding datatype. All such information is used as input features to predict the datatype of properties that are expected to have literal objects.

Table 6.12: Datatypes of dbp:Person-dbp:deathDate literals

\begin{tabular}{|l|l|l|l|l|}
\hline \multirow{2}{*}{ Datatype } & \multicolumn{2}{|c|}{$\begin{array}{c}\text { Objects } \\
(\mathbf{6 5 , 2 7 2})\end{array}$} & \multicolumn{2}{c|}{$\begin{array}{c}\text { Distinct Objects } \\
(\mathbf{3 2 , 4 4 9})\end{array}$} \\
\cline { 2 - 5 } & Count & $\mathbf{\%}$ & Count & $\mathbf{\%}$ \\
\hline xsd:date & 39,761 & 60.92 & 26,726 & 82.36 \\
\hline xsd:integer & 13,543 & 20.75 & 1,758 & 5.42 \\
\hline rdf:langString & 6,388 & 9.79 & 3,512 & 10.82 \\
\hline xsd:gMonthDay & 5,446 & 8.34 & 366 & 1.13 \\
\hline dt:second & 113 & 0.17 & 66 & 0.20 \\
\hline xsd:double & 20 & 0.03 & 20 & 0.06 \\
\hline dt:hour & 1 & 0.00 & 1 & 0.00 \\
\hline Total & $\mathbf{6 5 , 2 7 2}$ & $\mathbf{1 0 0}$ & $\mathbf{3 2 , 4 4 9}$ & $\mathbf{1 0 0}$ \\
\hline
\end{tabular}

String-based constraints: The value distribution analysis task in Chapter 5 extracts the statistics about the string lengths of string literal objects of each property. 


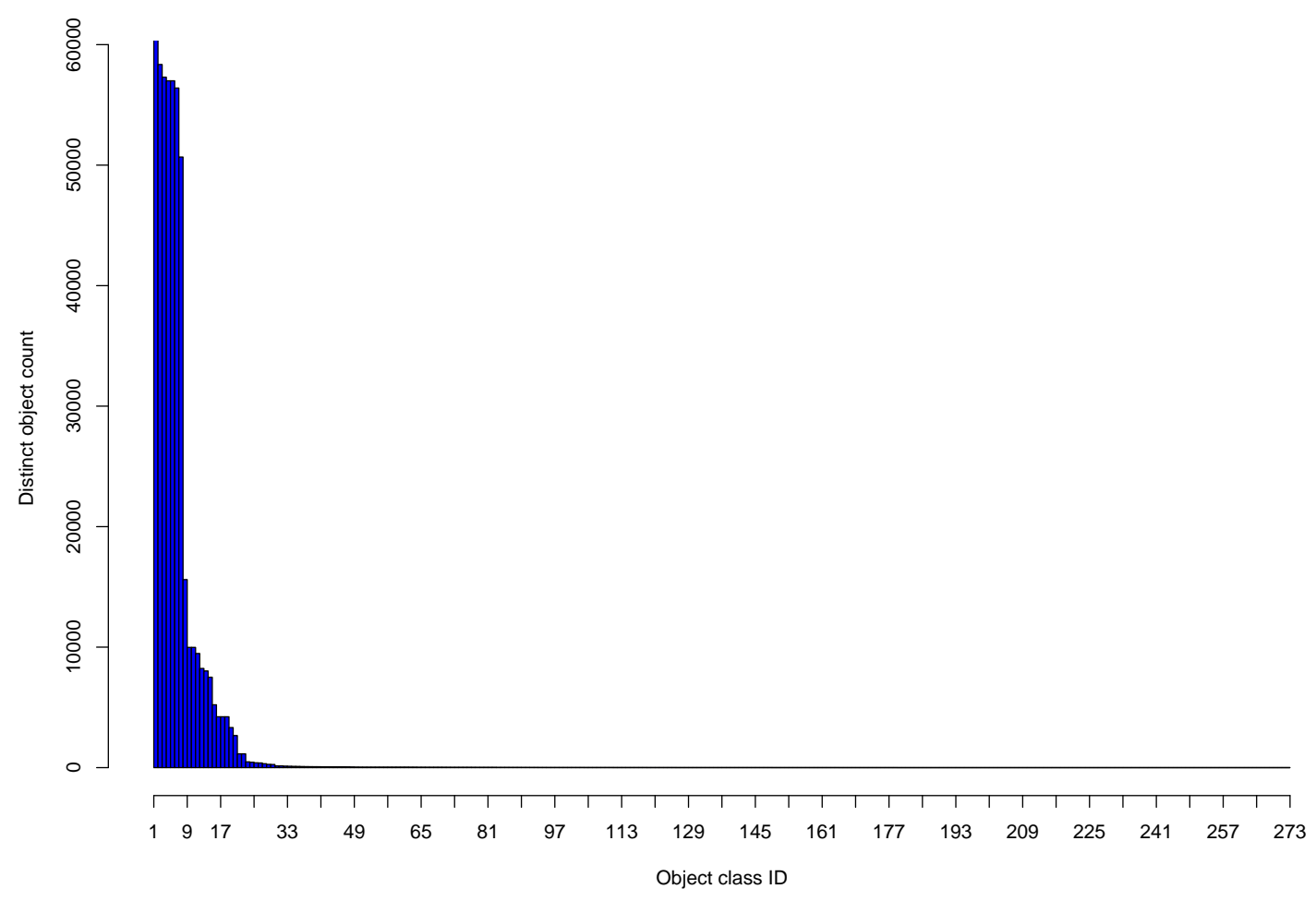

Figure 6.4: Histogram of object types of dbo:Person-dbp:birthPlace

When we explored the string lengths of different properties, we could identify two common scenarios. On the one hand, there are properties that have values of the same length or a set of small number of lengths, for example, codes with a predefined format and length. On the other hand, there are properties that have values of significantly variable lengths, for example, names of persons.

For example, Table 6.13 shows an example of the string length distribution of the dbo:iso6393code property in instances of the schema:Language class together with the frequency of the string length. This property belongs to the first type discussed before where the majority of the values have a length of 3. Table 6.14 shows an example of string length distribution of the foaf:name property in the instances of the foaf:Person class. In this case, the string length of the values is more variable. Nevertheless, as it is illustrated in Figure 6.5, there are some patterns that provide heuristics for identifying the min and max string lengths.

As discussed in the task formulation step of string-based constraints, we fist estimate several possible minimum and maximum string lengths based on the percentiles in the string length distributions (for example, as shown in Table 6.13 and 
Table 6.14). Then, to select one of the percentiles as the expected minimum and maximum string length, the number of distinct string lengths, the percentiles values, and other descriptive statistics are used as input features for predicting the min and max string lengths of strings.

Table 6.13: Frequency distribution of schema:Language/dbo:iso6393Code property.

\begin{tabular}{|l|l|l|}
\hline String Length & Frequency & $\%$ \\
\hline 3 & 6815 & 99.96 \\
\hline 4 & 3 & 00.04 \\
\hline
\end{tabular}

Table 6.14: Frequency distribution of foaf:Person-foaf:name property.

\begin{tabular}{|l|l|c|}
\hline String Length & Frequency & $\mathbf{\%}$ \\
\hline 1 & 79 & 0.00 \\
\hline 2 & 1373 & 0.09 \\
\hline 3 & 4615 & 0.29 \\
\hline \multicolumn{3}{|c|}{ other rows are omitted for brevity } \\
\hline 12 & 187,377 & 11.84 \\
\hline 13 & 194,081 & 12.27 \\
\hline 14 & 177,089 & 11.19 \\
\hline \multicolumn{3}{|c|}{ other rows are omitted for brevity } \\
\hline 181 & 1 & 0.00 \\
\hline 184 & 1 & 0.00 \\
\hline 197 & 1 & 0.00 \\
\hline
\end{tabular}

\subsubsection{Gold Standard Preparation}

Because we are mainly using supervised machine learning techniques for constraint generation tasks, an important step of the method is to generate a gold standard that can used for training and validation of the models build for constraint generation.

We propose to do this by presenting a sample of data to human annotators and by asking them to label the data correctly. In addition, explicit ontological axioms can be used for this purpose when they are available (e.g., cardinality constraints).

Furthermore, once a model is built, to verify the performance of the automated approach, the results of the models can be further validated by a human expert. This manual evaluation will provide insights on whether accuracy of the model calculated by automatic means using the gold standard is reasonable.

If a given dataset has significant amount of errors, there are different strategies 


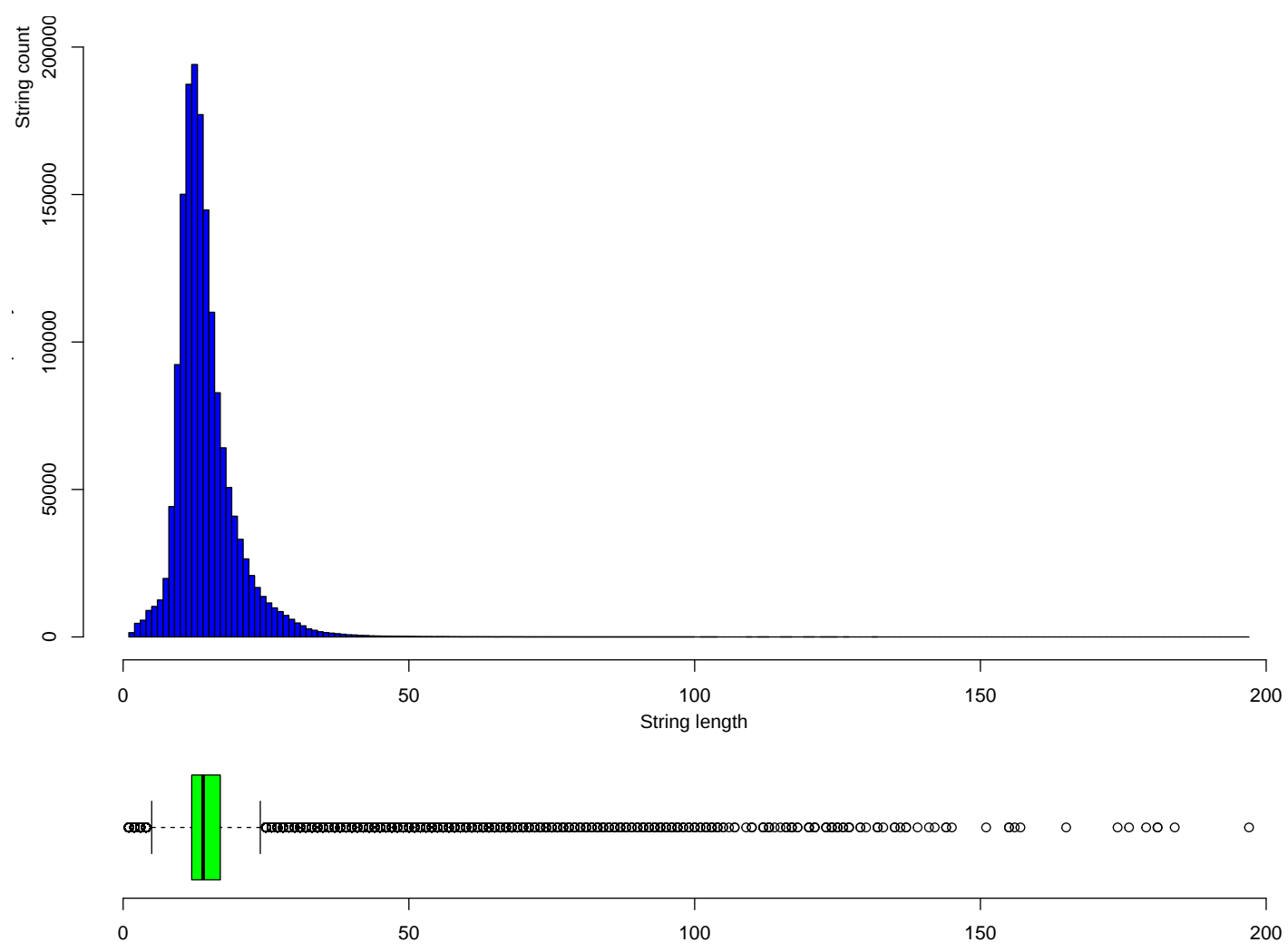

Figure 6.5: String length distribution of foaf:Person-foaf:name

for creating a training datasets. In the following, we present three common strategies in literature when dealing with such erroneous datasets [186]:

(i) Silver Standard: this strategy is based on the assumption that a given dataset is already of reasonable quality. The silver standard method is usually applied to measure the performance of knowledge graph by analysing how well relations in a knowledge graph can be replicated. Although this strategy is suitable for large-scale data, it can produce less reliable results [100, 131].

(ii) Gold standard: this strategy is based on turning observations in a set of gold data points by human annotators. In this work, we have used the gold standard approach. This approach can be used even when the dataset contains a lower quality because human annotators will be able to identify such data and eliminate them from the training data.

(iii) Partial gold standard: in this strategy, a small subset of external graphs, entities or relations are selected as validation criteria and they, then, are manually labelled [100]. This helps reducing the number of candidates that an annotator will process. 


\subsubsection{Algorithm Selection}

Once the previous two steps are performed, the next step is the algorithm selection step. In the algorithm selection, the goal is to select the most appropriate algorithm for each task that we identified in the first step. Because this step applies to all the tasks in a similar way, we will describe the algorithm selection step in a generic manner.

For selecting the most suitable algorithm, we use a representative sample of data from the problem domain by using the already annotated gold standards for each of the problems formulated before. We look at the accuracy of the models developed using different algorithms that are applicable for the problem. Following the state of the art, we define the accuracy as the number of correct predictions compared to all predictions made. In classical machine learning approaches for classification, it is known as classification accuracy. However, running an algorithm over a training dataset with different hyper-parameter settings will results in different model [35]. In particular, we are interested in selecting the best performing model. We used classifier accuracy to evaluate predictive performance of various models. More concretely, the steps of evaluating the predictive performance of a model are listed below:

1. The first step for building a good predictive model is to prepare the training data. By analysing the available data from the Linked Data profiling tasks discussed in Chapter 5, we noticed that in many cases there is an imbalance of different response variables (the expected output of prediction model) in training data. Because most algorithms can get affected by imbalanced data, it was important to address this issue. In our approach, we propose to use SMOTE (Synthetic Minority Over-sampling Technique) [49] for over sampling the rare response variables. The SMOTE function over-samples response variables by using bootstrapping and k-nearest neighbour to synthetically create additional observations of the response variable to have a balanced training dataset.

2. To reduce the variance of an accuracy score we need to ensure that each instance is used a equal number of times for training. We applied $k$-fold cross validation, where $k$ is the number of splits to make in the dataset. In this approach we choose value of $k=10$. This will result in splitting the dataset into 10 parts (10 folds) and run the algorithm 10 times. For each algorithm, training will be run on $90 \%$ of the data and testing on the $10 \%$. The algorithm will exchange which $10 \%$ of the data is tested with each run. In particular with a $k$ value of 10 will use each data instance as a training instance exactly nine times and as a test instance one time. The accuracy score will be how many correct predictions were made instead of a mean and a standard deviation.

3. It is important to establish the baseline performance on the given machine 
learning problem so that one can get a better insight of the performance of a given model. In this approach, we propose to use the ZeroR classification method as the baseline. ZeroR is a trivial classifier, but it gives a lower bound on the performance of a given dataset which should be significantly improved by more complex classifiers. As such, it is a reasonable test on how well the class can be predicted without considering the other attributes [231] .

4. Finally, we want to identify the machine learning algorithm that is best-suited for the problem at hand. In order to do so, we perform a comparison between selected algorithms. Based on the highest value of classifier accuracy, we selected the best performing model from the hypothesis space.

\subsubsection{Constraints and RDF Shape Generation}

As illustrated by Figure 6.3, the fifth step of the generic approach is constraint and RDF Shape generation. This is performed with the outputs of the previous three steps. The algorithm selected in the Step 3 is used with the features selected in Step 2 to build a model that can predict the constraints using the Linked Data profiling information. Once the constraint prediction models are built, constraints can be generated.

Cardinally constraints: In the classification task for cardinality constraints, we identify five main types of cardinality classes: MIN0, MIN1, MIN1+, MAX1, and MAX1+. Out of these, MIN0 (minimum zero values) and MAX1+ (maximum any value) do not put any constraints on the data, such that, any data will be valid for those cardinality types. Thus, if we detect those types we do not generate constraints. For other types, the corresponding SHACL property constraints are generated as illustrated by Listing 6.3.

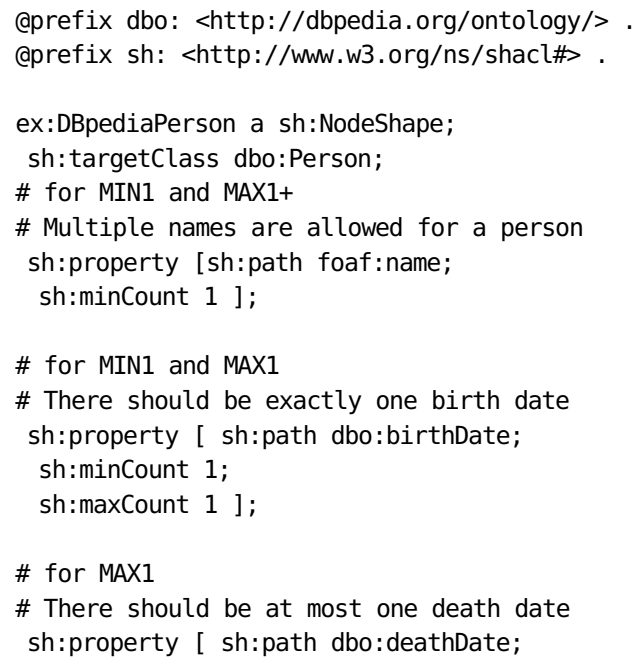


sh:maxCount 1 ].

Listing 6.3: An example of cardinality constraints

Range constraints: For range constraints, two types of constraints are generated. First, a node kind constraint is generated which defines if the objects of a given property are either IRI, Literal, BlankNode, or BlankNodeOrIRI. These node type constraints are represented as illustrated in Listing 6.4. Then, based on the node kind, data type constraints are generated which can be either a class for IRI objects or a datatype for literal objects.

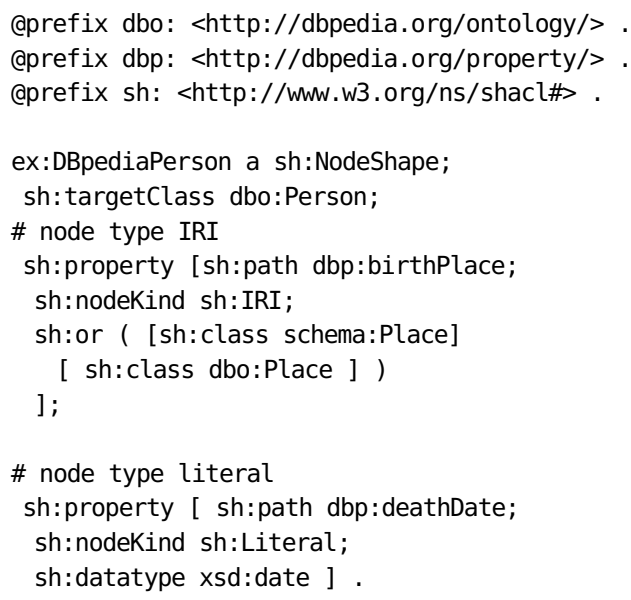

Listing 6.4: Node type constraints

String-based constraints: For string based constraints, two types of constraints are generated that are related to the minimum string length and the maximum string length as illustrated in Listing 6.5.

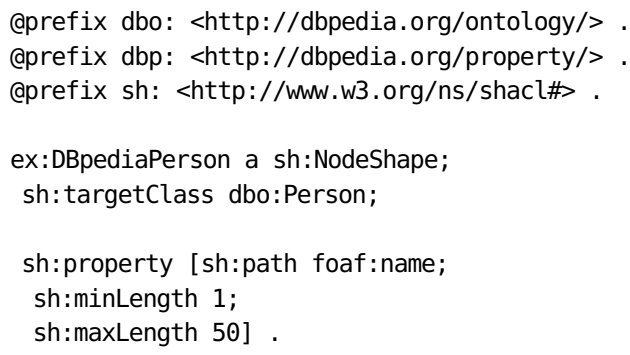

Listing 6.5: Node type constraints

The goal of the final step is to combine all the constraints related to a given class and to formulate an RDF Shape for the class. Previously, the constraints were generated for each class-property combination, for example, the cardinality constraints for foaf:name when associated with dbo:Person class. Similarly, constraints are generated to all properties associated with instances of dbo:Person class. All those 
constraints of a given class are combined together to generate the shape of the class. For example, Listing 6.6 illustrates a snippet from the Person RDF Shape.

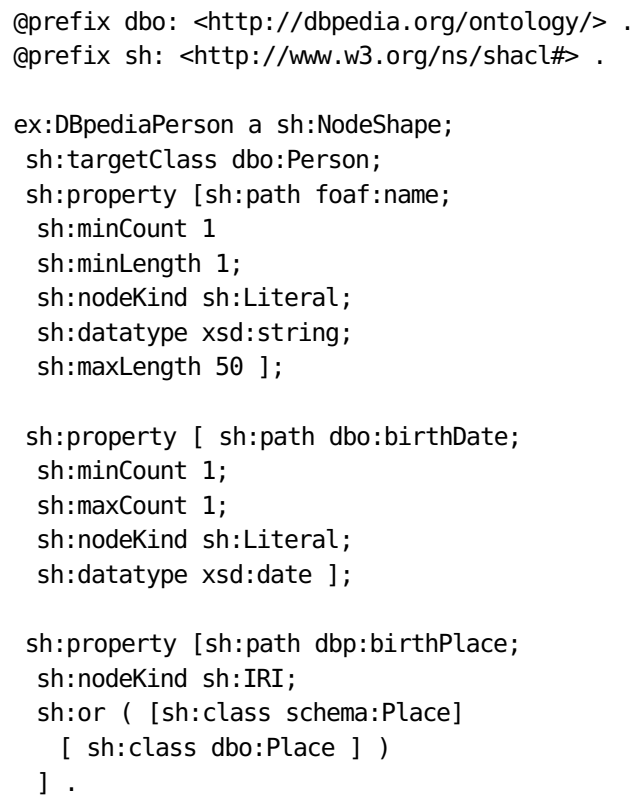

Listing 6.6: A snippet from the Person RDF Shape

\subsection{Future Work}

In this Chapter, we presented an automatic RDF Shape induction approach using Linked Data profiling information as input features. We have defined the approach in a generic way and showed how it can be implemented for three different types of constrains, namely: cardinality constraints, range constraints, and string-based constraints. As future work, we plan to use this approach for extracting other constraints which can be expressed through the W3C SHACL language. In particular, we will extend our implementation to other constraints such as property pair constraints, and value range constraints. Furthermore, we would like to facilitate a more efficient feature vectors for the feature engineering by proposing improved classification strategies. We perceive that the scalability of our approach can be increased by using incremental methods, as for example, monitoring the changes in the underlying datasets. 


\section{Chapter 7}

\section{Methods for Quality Assessment and Repair}

As discussed in Chapter 3, the fourth objective of this thesis is to develop methods and techniques for automatic quality assessment and repair using the data profiling metrics that were discussed in Chapter 5 and using integrity constraints represented as RDF Shapes that were discussed in Chapter 6. Aligned with the work discussed in the previous chapters, this chapter presents a set of methods and techniques for quality assessment and repair for distinct use cases related to a set of large datasets.

Concretely, we present four use cases involving Linked Data quality issues and propose methods for quality assessment and repair. These use cases cover Linked Data generation, storage, and exploitation phases. Furthermore, they cover both quality assessment and repair use cases in both industrial as well as public datasets as illustrated in Table 7.1.

Table 7.1: Quality assessment and repair use cases

\begin{tabular}{|l|l|l|l|l|l|}
\hline \multirow{2}{*}{ Use case } & \multicolumn{2}{c|}{ Purpose } & \multicolumn{2}{c|}{ Dataset Type } \\
\cline { 3 - 6 } & $\begin{array}{l}\text { Quality } \\
\text { Assessment }\end{array}$ & Repair & Public & $\begin{array}{l}\text { Enter- } \\
\text { prise }\end{array}$ \\
\hline I & Detection of inconsistent mappings & $\checkmark$ & & $\checkmark$ & \\
\hline II & Noise detection in dataset generation & $\checkmark$ & $\checkmark$ & & $\checkmark$ \\
\hline III & Repairing hidden links & $\checkmark$ & $\checkmark$ & $\checkmark$ & \\
\hline IV & Type completion & & $\checkmark$ & $\checkmark$ & \\
\hline
\end{tabular}




\subsection{Detection of inconsistent RDF mappings}

\subsubsection{Use Case}

A large number of RDF datasets are created by transforming non-RDF semi-structured or structured data sources into RDF. Such non-RDF data sources include relational databases, CSV files, key-value pairs, etc. A key input to this transformation process is a mapping that defines how to transform the non-RDF source data into RDF. Such mapping specifies how to map the non-RDF source schema elements into RDF vocabularies, and could possibly include other data transformations (e.g., for splitting a given attribute into multiple values or aggregating several attributes into a single value).

The first use case is focused on Linked Data generation processes using RDF mappings [61]. This use case is mainly motivated by the DBpedia dataset [17]. In large datasets such as DBpedia, the mappings are developed as a community effort with mapping editors of varying level of domain knowledge and expertise and these RDF mappings could have quality issues. Such issues have a significant impact on the quality of the generated datasets because one erroneous mapping could lead to a large number of erroneous triples in data. Thus, it is important to perform quality assessment of RDF mappings to identify erroneous mappings.

As discussed in Chapter 4, consistency is one of the important quality characteristic in Linked Data. One of the indicators of the consistency quality characteristic is the consistency of the mappings. This use case is focused on how to detect inconsistent RDF mappings that are used to generate Linked Data.

DBpedia, the main hub of the Linked Open Data Cloud, exposes data from Wikipedia as machine-readable Linked Data. Until 2011, only data from the English language Wikipedia was extracted but, since then, several "language-specific" DBpedia chapters were created for other Wikipedia languages. For example, the Spanish DBpedia $^{1}$ (esDBpedia) was created in 2012 and currently there are more than 15 other "language-specific" DBpedia chapters including Spanish, German, Dutch, Greek, Portuguese, and Italian DBpedia chapters.

The DBpedia data extraction process generates RDF data based on the mappings [199] that map: (1) Wikipedia infobox templates to classes of the DBpedia ontology, and (2) infobox template key-value pairs of each Wikipedia infobox template to ontology properties. In a large knowledge base such as DBpedia there is a considerably large number of mappings; for instance, DBpedia 2016-04 version has more than $5 \mathrm{~K}$ template mappings (for all languages) and a much larger number of infobox template key-value pairs. As these mappings are created by a diverse community of volunteers using crowd-sourcing techniques, it is frequent to have wrong

\footnotetext{
${ }^{1}$ http://es.dbpedia.org
} 
or inconsistent mappings. Notice that each incorrect mapping produces a plethora of incorrect data because there are thousands of infobox instances for a given infobox template. For instance, if the infobox template for Mountain, which contributes to the generation of 16 thousand Mountain instances in the English Wikipedia, has an incorrect mapping for one of its keys (e.g. mapping the property dbo:height instead of the property dbo:elevation for the key elevation), all the mountain instances would get an incorrect value for that property.

The wrong mappings can be due to many reasons. The DBpedia ontology is considerably large and evolves in a collaborative manner with significant additions and removals in each version and some mapping contributors do not have knowledge about all the 685 classes and $3500+$ properties of the ontology. Further, there are also language issues. Most of the contributors of the language-specific DBpedia chapters are non-native English speakers and they have influences from their native language when selecting mapping terms. For instance, while in the English DBpedia elevation of a mountain is mapped to the dbo:elevation property most languagespecific DBpedia instances map it to dbo:height which is not intended for mountains but for humans. Inconsistencies of the mappings (same semantic relation mapped to distinct properties) can also be caused by duplicate properties in large ontologies such as the DBpedia ontology. For instance, the DBpedia ontology has two properties dbo:formation Year and dbo:founding Year and both denote the year an organisation was established. As both terms are suitable for representing the relation, different DBpedia language-specific chapters use them in mappings in an inconsistent manner. In such cases, even though the generated data is not semantically incorrect, the proper reuse of data is hindered because it is hard to query the data (the users will have to use all the possible alternatives in queries) and hard to integrate data from multiple language-specific datasets.

Another problematic type of mappings is mapping more specific relations to generic properties in DBpedia. For instance, for describing the postal code of a city, some mappings use the generic property dbo:code instead of the more specific and most appropriate property dbo:postalCode. Such mappings also create inconsistencies among data from different language-specific DBpedia datasets and make queries across the DBpedia language-specific datasets harder. Thus, all these inaccuracies and inconsistencies in mappings either lead to incorrect data or causes inconveniences for querying or data integration. However, as these inaccuracies and inconsistencies are scattered over a large number of mappings, it is not feasible to analyse each of them manually by a set of experts. Further, most of such inconsistencies are not unveiled when they are inspected individually but rather uncovered only when they are compared to other similar mappings. 


\subsubsection{Approach}

The main objective of the method proposed in this section is to propose a data-driven method to detect the aforementioned mapping deficiencies automatically using the Linked Data profiling information discussed in Chapter 5. More concretely, the proposed approach includes: (1) an analysis of instance data from distinct languagespecific datasets using data profiling, and (2) an analysis of the ontological axioms of the DBpedia ontology. Our goal is to build a classifier using a set of features that can be used to identify such deficiencies.

In this work, we use the intuitive idea that when a given resource has the same object value for two distinct properties, there is a high probability of having a mapping inconsistency, i.e., the same relation is mapped to two distinct properties. For example, if the English and Spanish DBpedia have the same subject-object pair $<$ Mount_Everest, 8848> connected with two distinct properties dbo:elevation and $\mathrm{dbo}$ : height, it may be possible that both properties refer to the same relation. To identify such occurences, we use different language-specific DBpedia instances (for example, English DBpedia, Spanish DBpedia, Greek DBpedia, Dutch DBpedia) with similar data with equivalence relations (as owl:sameAs links) among them.

Nevertheless, we also take into account that the possibility of two distinct properties having the same value by coincidence (e.g., birthPlace and deathPlace of a person or largestCity and capital of a country) could have same subject and same object pairs quite frequently even though those relations are not semantically equivalent. We define a set of data-driven metrics taking all these aspects into account. Further, we also define a set of metrics based on the ontological axioms such as the domain and range of a given property and their hierarchical relationships to extract features that can help to determine if a given mapping is incorrect.

Our hypothesis is that we can develop a classifier capable of identifying incorrect mappings with high precision by using the features that we have defined in this study. In order to evaluate this hypothesis we have created a gold standard that can be used as training and test data by manually annotating DBpedia mappings pairs from multiple languages (English-Spanish, English-Greek, English-Dutch, SpanishGerman). The selection of the languages were driven by the availability of human annotators.

Figure 7.1 illustrates the overall approach that has been followed by the proposed method. In the case of DBpedia, both data, as well as RDF mappings modelled using the RML language [61], are represented in RDF. We propose to use the Linked Data profiling framework presented in Chapter 5 to derive a set of input features for a binary classifier that can distinguish inconsistent mappings from consistent mappings. 


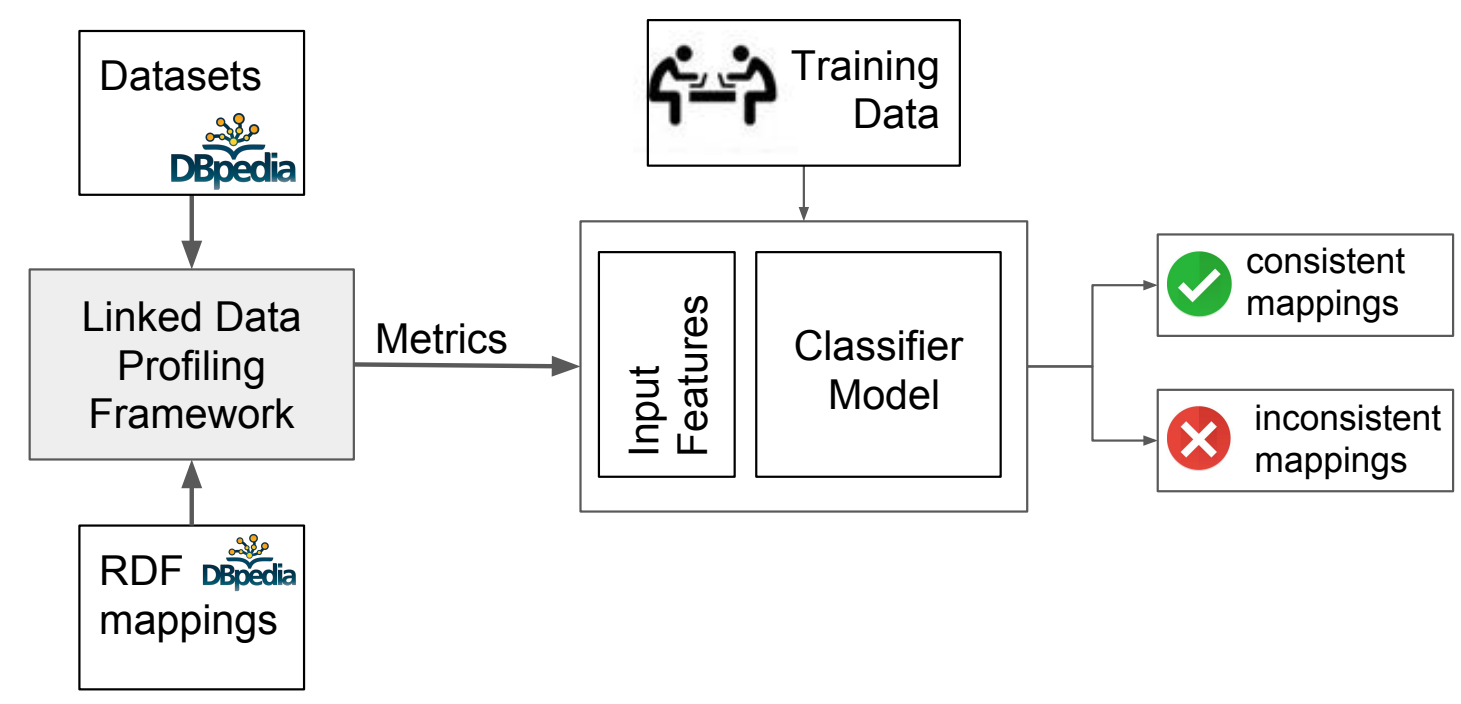

Figure 7.1: Detecting inconsistent mappings

\subsubsection{Inconsistent Mappings}

The main research problem studied when developing this method is "is it possible to automatically detect inconsistent mappings by analysing two knowledge graphs created using two sets of different mappings?". We assume that the knowledge graphs have an overlap, i.e., some entities are described in both, and the coreferences (owl:sameAs links) can be resolved.

In the DBpedia use case, we can find a large number of datasets that contain similar data, i.e., the language-specific DBpedia knowledge bases and a large portion of the entities present in those knowledge bases are linked by equivalence relationships based on the manually annotated wikilinks contributed by the Wikipedia community. For instance, the entity dbr:Mount_Everest is included in more than 15 other DBpedia language-specific datasets, and the coreferences (i.e., the links that refer to the same entity) annotated using the owl:sameAs relation [103]. All these entities that describe Mount Everest in different DBpedia datasets such as English DBpedia, Spanish DBpedia, Greek DBpedia, and German DBpedia mostly contain similar information about it such as its location, elevation, important climbers, etc. Thus, as it can be seen in Table 7.2, such relations are mapped correctly to the same corresponding property in all DBpedia language-specific datasets.

However, in some cases, due to the errors in the mappings such relations can be mapped to properties not intended for the given relation. For instance, Table 7.2 shows the property that corresponds to the elevation of the Month Everest in each dataset. As it can be seen from the table, while three of the datasets have mapped 


\begin{tabular}{|c|c|c|c|}
\hline DBpedia & Subject & Predicate & Object \\
\hline \multirow{2}{*}{ English } & \multirow{2}{*}{ dbr:Mount_Everest } & geo:long & 86.925278 \\
\hline & & dbo:mountainRange & dbr:Himalayas \\
\hline \multirow{2}{*}{ Spanish } & \multirow{2}{*}{$\begin{array}{l}\text { dbr-es:Monte_Everest } \\
\text { (owl:sameAs } \\
\text { dbr:Mount_Everest) }\end{array}$} & geo:long & 86.925278 \\
\hline & & dbo:mountainRange & $\begin{array}{l}\text { dbr-es:Himalayas } \\
\text { (owl:sameAs } \\
\text { dbr:Himalayas) }\end{array}$ \\
\hline \multirow{2}{*}{ Greek } & \multirow{2}{*}{$\begin{array}{l}\text { dbr-el:'E } \beta \varepsilon \rho \varepsilon \sigma \tau \\
\text { (owl:sameAs } \\
\text { dbr:Mount_Everest) }\end{array}$} & geo:long & 86.925278 \\
\hline & & dbo:mountainRange & $\begin{array}{l}\text { dbr-el:I } \mu \alpha \lambda \alpha^{\prime} \iota \alpha \\
\text { (owl:sameAs } \\
\text { dbr:Himalayas) }\end{array}$ \\
\hline \multirow{2}{*}{ German } & \multirow{2}{*}{$\begin{array}{l}\text { dbr-de:Mount_Everest } \\
\text { (owl:sameAs } \\
\text { dbr:Mount_Everest) }\end{array}$} & geo:long & 86.925278 \\
\hline & & dbo:mountainRange & $\begin{array}{l}\text { dbr-de: } \\
\text { Mahalangur_Himal } \\
\text { (owl:sameAs } \\
\text { dbr:Himalayas) }\end{array}$ \\
\hline
\end{tabular}

Figure 7.2: Data from correct and consistent mappings

that relation to dbo: elevation property, the Spanish DBpedia dataset has mapped it to dbo: height. In our work, we use a set of features extracted from the two datasets to identify such incorrect mappings.

In DBpedia, this data is generated from Wikipedia by mapping Wikipedia infobox keys to DBpedia ontology properties. DBpedia maintains mappings for each languagespecific Wikipedia template and those mappings are improved over time by contributors adding mappings to common keys in those infobox templates. At the moment, these mappings are maintained in a specific wiki ${ }^{2}$ and the DBpedia contributors with access to the mapping wiki maintain and improve the mappings on a regular basis. However, as those mappings are created by a diverse community, inaccuracies and inconsistencies are introduced during this process. In this work, we categorise mappings firstly into two categories: correct and incorrect mappings.

During the evaluation of this method, we test the hypothesis is that we can develop a classifier capable of identifying incorrect mappings with high precision by using the features that we have defined in this study. In the next section, we discuss an approach for developing such classifier and how to evaluate it.

\footnotetext{
${ }^{2}$ See http://mappings.dbpedia.org
} 
Table 7.2: Data from an incorrect mapping

\begin{tabular}{|l|l|l|l|}
\hline DBpedia Dataset & Subject & Predicate & Object \\
\hline English & dbr:Mount_Everest & dbo:elevation & 8848 \\
\hline Spanish & dbr-es:Monte_Everest & dbo:height & 8848 \\
\hline Greek & dbr-el: $\epsilon \beta \epsilon \rho \sigma \tau$ & dbo:elevation & 8848 \\
\hline German & dbr-de:Mount_Everest & dbo:elevation & 8848 \\
\hline English & dbr:Spanish_language & dbo:iso6393Code & spa \\
\hline Spanish & dbr-es:Idioma_español & dbo:iso6393Code & spa \\
\hline German & dbr-de:Spanische_Sprache & dbo:code & spa \\
\hline
\end{tabular}

\subsubsection{Inconsistent Mapping Prediction Method}

The approach that was followed to address this problem of inconsistent mappings is to build a predictive model that can identify inconsistent mappings using the Linked Data profiling information. In order to use machine learning techniques, we have formulated the problem of detecting inconsistent mappings as a binary classification task. This section describes the feature engineering for creating the predictive model, as well as the model preparation process.

\section{Feature descriptions}

The features that we used for building the model are of two types: (a) instance-based features, and (b) schema-based features. We will define the features using two RDF graphs, graph $G_{i}$ where $\left\langle S_{i}, P_{i}, O_{i}\right\rangle \in G_{i}$ and graph $G_{i}$ where $\left\langle S_{j}, P_{j}, O_{j}\right\rangle \in G_{j}$.

Instance-based features are extracted from $A B o x$ information in a data-driven manner. The key advantage of such features is that they can be extracted even when no schema information is available. There are four direct features, denoted by M1-M4 and four derived features, denoted C1-C4. Direct features can be any whole number; derived features always have a normalised range of $[0-1]$.

$M_{1}\left(G_{i}, G_{j}, P_{i}, P_{j}\right): M_{1}$ is defined as the count of $S_{i}$ resources i.e. $\left\langle S_{i}, P_{i}, O_{i}\right\rangle \in G_{i}$, $\left\langle S_{j}, P_{j}, O_{j}\right\rangle \in G_{j}$, and $S_{i}$ is same as or equivalent to $S_{j}$. This gives the frequency of resources having property $P_{i}$ in graph $G_{i}$ while having property $P_{j}$ in $G_{j}$. The rationale behind this feature is to check how probable is that a resource will have property $P_{j}$ in $G_{j}$ given that the resource has $P_{i}$ in $G_{i}$. For example, if the elevation of a mountain is mapped to dbo:elevation in English DBpedia, and wrongly mapped to dbo:height in Spanish DBpedia, the instances of mountains having dbo:elevation in English DBpedia and dbo:height in Spanish. Our intuition is if $P_{j}$ is wrongly mapped in $G_{j}$ (to denote $P_{i}$ ) this number should be high.

$M_{2}\left(G_{i}, G_{j}, P_{i}, P_{j}\right): M_{2}$ is defined as the number of $\left\langle S_{i}, O_{i}\right\rangle$ pairs i.e. $\left\langle S_{i}, P_{i}, O_{i}\right\rangle \in$ 
$G_{i},\left\langle S_{j}, P_{j}, O_{j}\right\rangle \in G j, S_{i}$ is equivalent to $S_{j}$, and also $O_{i}$ is equivalent to $O_{j}$. Because $M_{2}$ only counts occurrences where the objec is also the same, it is even stronger indication that two properties refer to the same relation (see, Table 7.2). Our intuition is that when $M_{2}$ is high, there is a higher-probability that those properties may refer to the same relation. For example, if we take $M_{2}(E N, E S$, dbo:elevation,dbo:height), there are 5108 resources with the same object value in two graphs, giving an indication that they might refer to the same relation and one mapping is inconsistent.

$M_{3}\left(G_{i}, G_{j}, P_{i}, P_{j}\right): M_{3}$ is defined as the number of $S_{i}$ resources i.e. $\left\langle S_{i}, P_{i}, O_{i}\right\rangle \in G_{i}$ and $\left\langle S_{j}, P_{j}, O_{j}\right\rangle \in G_{j}$ where $S_{i}$ is equivalent to $S_{j}$ but $O_{i}$ is different from $O_{j}$. This feature looks for the opposite of $M_{2}$. The rationale is that it is possible to have false positives in $M_{2}$, for example, people are born and have died in the same place by coincidence (dbo:birthPlace/dbo:deathPlace) or actors who are also directors in a given film (dbo:directedBy/dbo:actor). Thus, in this feature we count the number of counter examples. Our intuition is that when we find few matches in $M_{2}$ by coincidence, $M_{3}$ should be able to find a reasonable amount of counterexamples.

$M_{4}\left(G_{i}, P_{i}, P_{j}\right): M_{4}$ is defined as the number of $S_{i}$ resources in graph $G_{i}$ that contains both property $P_{i}$ and $P_{j}$ simultaneously in the same graph. The rationale for this feature is similar to $M_{3}$. The intuition is that this is higher when the two properties denote two distinct relations than when they denote the same. For example, more resources have both dbo:birthPlace and dbo:deathPlace properties in the same resource simultaneously (denoting two distinct relations) compared to dbo:elevation and dbo:height in same resource.

The derived features are calculated by normalising $M_{2}, M_{3}$, and $M_{4}$ using $M_{1}$ i.e. $C_{1}=M_{2} / M_{1}, C_{2}=M_{3} / M_{1}$, and $C_{3}=M_{4} / M_{1}$.

Schema-based features are extracted from TBox information. When we manually analyzed mappings, we found that it is common to have generic properties in some wrong mappings, for instance, using dbo:code instead of dbo:postalCode. Further, we noticed wrong mappings could also be due to duplicated properties (for the same relation), for example, dbo:founding Year and dbo:formation Year. Schema-based features try to capture hints for such cases. There are 11 schema-based features: TB1-TB11.

$T B_{1}$ checks if the property $P_{i}$ is a subproperty of $P_{j}$ and $T B_{2}$ checks vice versa, i.e., $P_{j}$ is a subproperty of $P_{i}$. T $B_{3}$ checks if the classes corresponding are the same in both graphs. $T B_{4}$ checks if the class in $G_{i}$ is a subclass of the class in $G_{j}$ and $T B_{5}$ checks vice versa. $T B_{6}$ checks if the domains of $P_{i}$ and $P_{j}$ are the same. $T B_{7}$ $\operatorname{domain}\left(P_{i}\right)$ is subclass of $\operatorname{domain}\left(P_{j}\right)$ and $T B_{8}$ checks vice versa. Similarly, $T B_{9}$ checks if the ranges of $P_{i}$ and $P_{j}$ are the same. T $B_{10}$ checks range $\left(P_{i}\right)$ is subclass of $\operatorname{range}\left(P_{j}\right)$ and $T B_{11}$ checks vice versa. 


\subsubsection{Training the Prediction Model}

Because we are using binary classification, which is a supervised learning technique, it is necessary to collect annotations to train the learning algorithms.

We have asked experts from 4 DBpedia chapters to manually inspect mappings. For selecting the mappings that can possibly contain errors, we have used our previous assumption that when there is a high number of same subject and same object combinations with different properties in two different datasets of DBpedia, this could be because of a wrong mapping. Thus, we selected such mappings from 4 combinations of DBpedia datasets (EN-ES, EN-DE, EN-NL, EN-GR) and asked the language-pair experts to annotate if the mappings were correct or not. The instructions provided to the language-pair experts are available online in the following $\operatorname{link}^{3}$.

To facilitate the annotation, for each mapping we provided the Wikipedia infobox template name, infobox key, and the property it is mapped to for each of the languages. A snippet from the English-Spanish annotation table is shown in Table 7.3.

\subsubsection{Usage of the Prediction Model}

In this section, we explain the usage of the prediction model for identifying inconsistent mappings using the input features described in Section 7.1.4. For the sake of clarity, we will describe the process for the English-Spanish (EN-ES) case. We distinguish between two types of properties that can used in the mappings: owl:ObjectProperty and owl:DatatypeProperty [211]. In the case of object properties the objects of the generated triples would be IRIs whereas in the case of datatype properties the objects would be literals. When building the predictive mappings, we made this distinction because of the mapping inconsistencies could have different characteristics in mappings containing object properties and datatype properties. Thus, a model for each type could yield better performance.

We start with the mappings containing datatype properties (literal objects), in which 226 annotations have been provided manually by contributors from our institutions, fluent in both languages. Then, we trained a predictive model with these annotations. The training set contains 182 mappings annotated as "Correct" and 44 as "Incorrect". Therefore, the simplest classifier (known as ZeroR), which assigns the most popular class value, has an accuracy of $64.29 \%$. This classifier establishes the baseline value that must be enhanced by our model. In the evaluation section, we present a rigorous evaluation of the proposed prediction model

In the cases of mappings with object properties (IRI objects), the dataset (80 instances, 71 Correct and 9 Incorrect). If we apply this predictive model to mappings

\footnotetext{
${ }^{3}$ http://goo.gl/M1go5S
} 
Table 7.3: A snippet from English-Spanish mapping annotation

\begin{tabular}{|c|c|c|}
\hline \multicolumn{3}{|r|}{ Annotation I } \\
\hline \multirow{2}{*}{ Template } & en & Infobox_French_commune \\
\hline & es & Ficha_de_entidad_subnacional \\
\hline \multirow{2}{*}{ Attribute } & en & elevation_m t \\
\hline & es & elevación_media \\
\hline \multirow{2}{*}{ Property } & en & dbo:elevation \\
\hline & es & dbo:height \\
\hline Annotation & \multicolumn{2}{|r|}{$\begin{array}{l}\text { Inconsistent mapping (note that in this is case, dbo:elevation } \\
\text { was the expected property to be used with mountains etc.) }\end{array}$} \\
\hline \multicolumn{3}{|r|}{ Annotation II } \\
\hline \multirow{2}{*}{ Template } & en & Infobox_company \\
\hline & es & Ficha_de_organización \\
\hline \multirow{2}{*}{ Attribute } & en & foundation \\
\hline & es & fundación \\
\hline \multirow{2}{*}{ Property } & en & dbo:foundingYear \\
\hline & es & dbo:formationYear \\
\hline Annotation & \multicolumn{2}{|r|}{$\begin{array}{l}\text { Inconsistent mapping (note that sometimes the ontology has } \\
\text { similar properties for the same thing but this is still a wrong } \\
\text { mapping) }\end{array}$} \\
\hline \multicolumn{3}{|r|}{ Annotation III } \\
\hline \multirow{2}{*}{ Template } & en & Infobox_mountain \\
\hline & es & Ficha_de_montaña \\
\hline \multirow{2}{*}{ Attribute } & en & elevation_m \\
\hline & es & elevación \\
\hline \multirow{2}{*}{ Property } & en & dbo:elevation \\
\hline & es & dbo:prominence \\
\hline Annotation & \multicolumn{2}{|c|}{ Inconsistent mapping } \\
\hline \multicolumn{3}{|c|}{ Annotation IV } \\
\hline \multirow{2}{*}{ Template } & en & Infobox_album \\
\hline & es & Ficha_de_álbum \\
\hline \multirow{2}{*}{ Attribute } & en & artist \\
\hline & es & productor \\
\hline \multirow{2}{*}{ Property } & en & dbo:artist \\
\hline & es & dbo:producer \\
\hline Annotation & \multicolumn{2}{|r|}{$\begin{array}{l}\text { Consistent(it happens that the producer is the same person } \\
\text { as the artist in some albums by coincidence) }\end{array}$} \\
\hline
\end{tabular}


Table 7.4: Summary of the results of applying the predictive model (EN-ES literals) to the IRIs dataset

\begin{tabular}{ll}
\hline Correctly Classified Instances & $12(85.7143 \%)$ \\
Incorrectly Classified Instances & $2(14.2857 \%)$ \\
Kappa statistic & 0.6889 \\
Mean absolute error & 0.2479 \\
Root mean squared error & 0.3102 \\
Total Number of Instances & 14 \\
\hline
\end{tabular}

Table 7.5: Detailed accuracy data and confusion matrix for the predictive model (EN-ES literals) on the IRIs dataset

\begin{tabular}{|c|c|c|c|c|c|c|c|c|c|}
\hline \multirow[t]{3}{*}{ Accuracy } & TP Rate & FP Rate & Precision & Recall & F-Measure & $\mathrm{MCC}$ & ROC Area & PRC Area & Class \\
\hline & 0.800 & 0.111 & 0.800 & 0.800 & 0.800 & 0.689 & 0.978 & 0.967 & Incorrect \\
\hline & 0.889 & 0.200 & 0.889 & 0.889 & 0.889 & 0.689 & 0.978 & 0.989 & Correct \\
\hline Avg. & 0.857 & 0.168 & 0.857 & 0.857 & 0.857 & 0.689 & 0.978 & 0.981 & \\
\hline \multicolumn{10}{|l|}{$\begin{array}{l}\text { ma- } \\
\text { trix }\end{array}$} \\
\hline & & $\begin{array}{l}\text { Pred. } \\
\text { Correct }\end{array}$ & $\begin{array}{l}\text { Pred. } \\
\text { Incorrect }\end{array}$ & & & & & & \\
\hline \multicolumn{2}{|c|}{ True Correct } & 8 & 1 & & & & & & \\
\hline \multicolumn{2}{|c|}{ True Incorrect } & 1 & 4 & & & & & & \\
\hline
\end{tabular}

where the generated objects are IRIs, the accuracy (correctly classified instances) is $95.00 \%$, very similar to the $93.36 \%$ achieved when objects are literals. Notice that this IRIs dataset, or any other IRI dataset, has not been "seen" by the model in its training.

\section{Prediction on datasets in other languages}

It would be great to have a unique model (trained with data annotated in a specific language) capable of predicting incorrect mappings not only in its own language but in other languages. This would be some sort of multilingual predictor. Notice that the manual annotation of each dataset is a high-specialised task that requires humans with excellent knowledge of the two languages involved. A unique model would save much human work.

We have applied this specific predictive model (EN-ES-lit) to the dataset ESDE-IRI containing 110 annotations. The accuracy (correctly classified instances) is $87.28 \%$, very similar to the $85.71 \%$ achieved for EN-ES-IRIs, but not as good as the EN-ES-lit with $93.36 \%$. The dataset is publicly available at https://www. openml . 
Table 7.6: Summary of the prediction accuracy for other language-pairs.

\begin{tabular}{|c|c|c|c|c|c|c|c|c|c|}
\hline & \multicolumn{2}{|c|}{ EN-ES } & \multicolumn{2}{|c|}{ ES-DE } & \multicolumn{2}{|c|}{ EN-NL } & \multicolumn{2}{|c|}{ EN-GR } \\
\hline & & lit & IRI & lit & IRI & lit & IRI & lit & IRI \\
\hline \multirow{2}{*}{ Accuracy } & Ad hoc model & \multirow{2}{*}{$93.36 \%$} & $95.00 \%$ & N.A. & $96.36 \%$ & $71.08 \%$ & $\sim 94 \%$ & $73.02 \%$ & $89.71 \%$ \\
\hline & En-ES-lit model & & $65.00 \%$ & N.A. & $87.28 \%$ & $61.45 \%$ & $67.86 \%$ & $77.78 \%$ & $88.24 \%$ \\
\hline \multirow{3}{*}{ Annotations } & Total instances & 211 & 80 & & 110 & 83 & 28 & 63 & 68 \\
\hline & 'Correct' instances & 175 & 71 & & 102 & 35 & 9 & 33 & 44 \\
\hline & 'Incorrect' instances & 36 & $\overline{9}$ & & 8 & 48 & 19 & 30 & 24 \\
\hline \multicolumn{2}{|c|}{ Number of mappings } & 799 & 4979 & & 4999 & 1329 & 4971 & 328 & 2785 \\
\hline
\end{tabular}

\section{org/d/40743.}

However, the English-Dutch literals (EN-NL-lit), with 83 annotations (35 Incorrect, 48 Correct) has an accuracy of only $61.45 \%$ If we create a predictive model for this data we get an accuracy of $71.08 \%$.

For Dutch (EN-NL-IRIs), with 28 annotations (19 Incorrect, 9 Correct) has an accuracy very low as well, only $67.86 \%$. A predictive model with this dataset would get an accuracy of $100 \%$. A detailed analysis, changing the aleatory seed, produces accuracy values around $94 \%$. This dependency on the seed indicates that the number of instances is too low.

For EN-GR-lit, with 64 annotations (30 Incorrect, 33 Correct) we get an accuracy of $77.78 \%$. If we create a predictive model for this data we get an accuracy of $73.02 \%$. All this information is condensed in table 8.25 , were we can see that for the 4 language-pairs studied the model for IRIs is always better than the model for literals. Besides, we can see that the EN-ES-lit model is good predicting incorrect mappings for some language-pairs (such as ES-DE-IRI or EN-GR-IRI), but not so good for another language-pairs (such as EN-NL-lit or EN-NL-IRI). Our conclusion is that it is not feasible to have a unique predictive model for all the language-pairs.

\subsubsection{Improving the Model}

As we have discussed in the previous sections, the prediction of inconsistent mappings can be useful for identifying such mappings and correcting them. Because each mapping generates a large number of triples in the dataset, each corrected mapping will contribute to a significant improvement of the quality of the dataset. Nevertheless, the proposed method requires multilingual domain experts to provide training data for generating the models. To that end, we have proposed an online web application that manages the two human profiles involved. On the one hand, it allows multilingual domain experts to annotate the mappings as discussed in the previous sections and enrich the training dataset. The domain experts who will act as annotators will be provided with a pair of mappings which generates at least 20 triples with same subject and same object pairs but with a different property in each dataset as illustrated in Figure 7.3. Based on the information from each Wikipedia infobox including the 
template and attribute and some examples from each DBpedia dataset, the annotators vote if the properties mapped in each DBpedia (English and Spanish in this example) are consistent or inconsistent. To perform this task, annotator should be fluent in both languages of the mapping pair and should have some knowledge about the DBpedia ontology. Currently, it is used by the members of each DBpedia language chapter who are fluent in another language. Multiple votes are gathered in order to check the inter-annotator agreement and when there are more than 5 annotators and the majority votes are $80 \%$ or above, the annotated mapping is added to the training data.

\begin{tabular}{|c|c|c|c|}
\hline $\begin{array}{l}\text { Infobox } \\
\text { Attribute } \\
\text { Property }\end{array}$ & $\begin{array}{l}\text { Infobox_count } \\
\text { official_langua } \\
\text { dbo:language }\end{array}$ & $\begin{array}{l}\text { Ficha } \\
\text { idiome } \\
\text { dbo:o }\end{array}$ & $\begin{array}{l}\text { es } \\
\text { país } \\
\text { cial } \\
\text { IlLanguage }\end{array}$ \\
\hline CONSIS & TENT MAPPING & INCONSISTENT MAPPING & HELP \\
\hline \multicolumn{4}{|c|}{ Example triples (EN | ES): } \\
\hline \multicolumn{4}{|c|}{$\underline{\text { dbr:Costa Rica - dbo:language | dbo:officialLanguage - dbr:Spanish language }}$} \\
\hline \multicolumn{4}{|c|}{ dbr:Colombia - dbo:language | dbo:officialLanguage - dbr:Spanish language } \\
\hline \multicolumn{4}{|c|}{ dbr:Ecuador - dbo:language | dbo:officialLanguage - dbr:Spanish language } \\
\hline \multicolumn{4}{|c|}{ Other users voted: } \\
\hline \multicolumn{2}{|c|}{ Consistent: 0} & Inconsistent: 4 & \\
\hline Prediction & Model Classified & 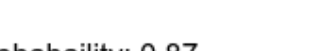 & \\
\hline
\end{tabular}

Figure 7.3: A screenshot from domain expert annotator web app

This process will make it possible to improve the model with more training data which will lead to better classification results. On the other hand, this tool allows mapping editors who are involved in the mapping creation process to exploit the predictions made by the model and to correct the inconsistent mappings in DBpedia. This is done by providing the mapping editors with a ranking of the most problematic mappings detected by the models for each language pair, as well as which are the conflictive properties so that the mapping editors can take necessary corrective actions. 


\subsection{Noise Detection in Dataset Generation}

\subsubsection{Use Case}

Linked Data can be generated using various different approaches. In the previous section, we focused on DBpedia, which is a dataset that is generated using RDF mappings between the Infobox-attribute pairs in the source data and classes and properties of the DBpedia ontology. In the second use case, we focus on datasets and knowledge bases created from natural language copra using information extraction tools. More specifically, we focus on an enterprise knowledge graph generated by companies for their internal use. In this section, we propose a technique for identifying noise in knowledge graphs generated using information extraction and improving the quality of such knowledge graphs by removing the noise.

Datasets, also commonly known as Knowledge Graphs (KGs), are key components of most modern artificial intelligence and cognitive applications. These datasets are largely constructed from textual corpora using automatic information extraction techniques. The output of such automatic techniques contains a set of high quality triples along with a set of erroneous triples which should be considered as noise as illustrated in Figure 7.4. Thus, in order to have a high quality datasets, noise detection and removal become essential steps.

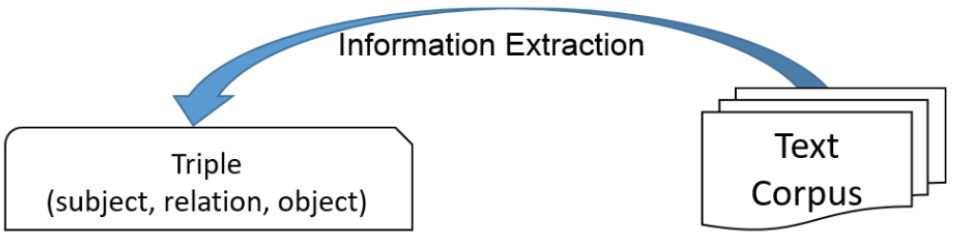

High Quality Facts Noise

\begin{tabular}{|l|l|l|}
\hline Subject & Relation & Object \\
\hline New York & locatedAt & United States \\
\hline Boston & locatedAt & India \\
\hline Barack Obama & siblingOf & Hawaii \\
\hline Barack Obama & spouseOf & Michelle Obama \\
\hline family & residesIn & New York \\
\hline
\end{tabular}

Figure 7.4: Information extraction output

Most of the current noise detection in RDF datasets is done with human supervision. For instance, large datasets such as YAGO [197], DBpedia [17], or Wikidata [227] 
use human contributors to verify the correctness of a given fact. This is a timeconsuming task and requires a lot of human effort for large datasets. Furthermore, in some cases this means that the humans performing the task will have to have some expert knowledge of the domain or the Semantic Web standards and good practices. Public datasets largely solve this issue using crowd-sourcing [5, 141]. Nevertheless, crowd-sourcing might not be a viable solution for enterprise datasets, due to both privacy issues as well as due to the need to rapidly create datasets from a large number of distinct corpora. Thus, there is a need for automatic techniques for detecting noise in datasets. Thus, our goal is to propose a method that is more suitable for enterprise datasets.

\subsubsection{Approach}

As illustrated in Figure 7.5, in the method presented in this section, we propose to detect noise in datasets generated using information extraction using the information from Linked Data profiling metrics and RDF Shapes. In order to do so, we first propose to transform the information extraction output, which generally is represented as a textual 3-tuple. Once the RDF data is generated, the techniques discussed in previous chapters are used to generate the Linked Data profiling metrics and RDF Shapes for the RDF data. The goal of the method is to use this information to build noise detection filters that can distinguish noise from high quality facts. Rather than building a single noise detection filter, in our approach we propose to identify different types of noise and build a noise detection filter for each type of noise.

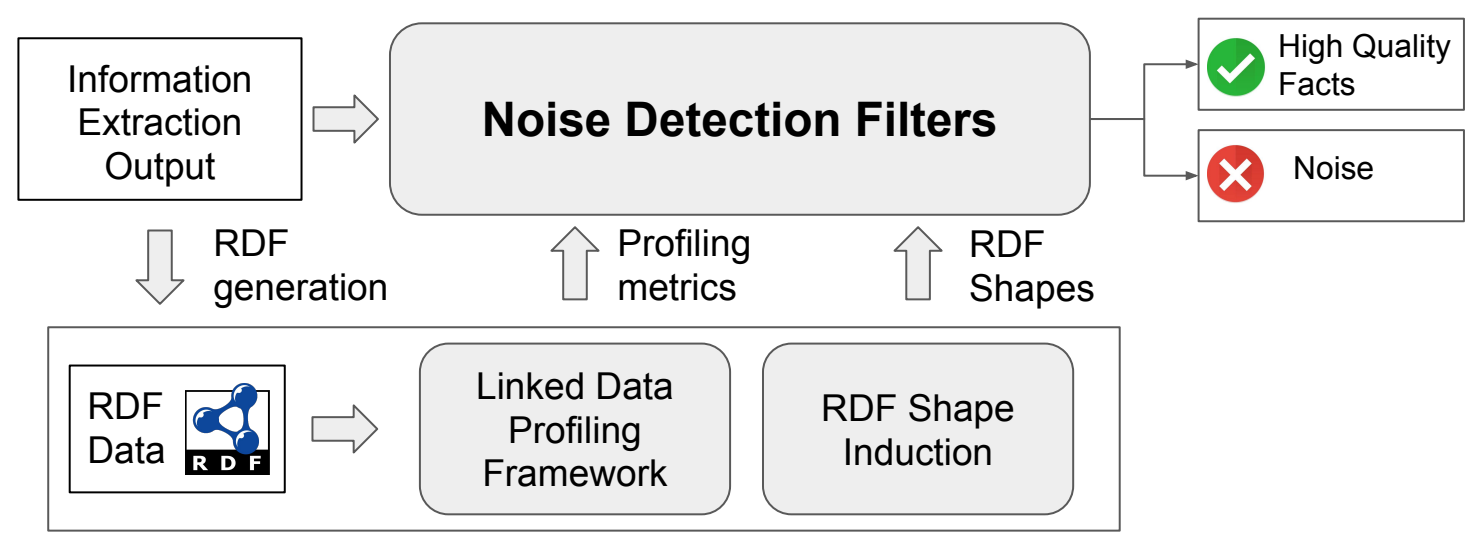

Figure 7.5: The noise detection workflow 


\subsubsection{Types of Noise}

Current automatic noise detection techniques are focused on factual correctness of triples. In this work, we discuss the need for different types of noise in datasets created from textual corpora using information extraction techniques and how to detect those specific types of noise.

Based on the analysis of the output of a commercial information extraction framework, which parses text corpora and produces triples, we defined the categories in Figure 7.6. Out of those, we identify Factual True triples as the most relevant and Inconsistent, Generic, Factual False categories as noise.

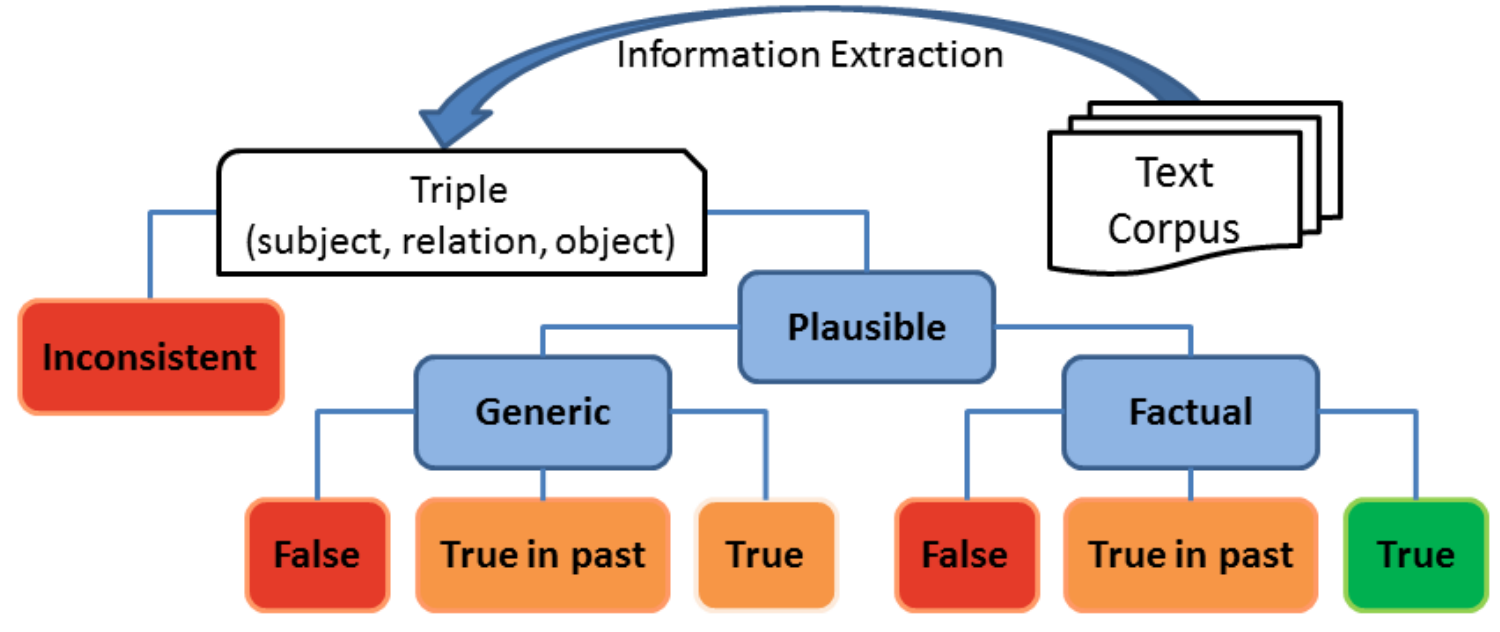

Figure 7.6: Types of noise

Inconsistent triples contradict the domain model they represent. As such, these triples are completely implausible and meaningless. For instance, (Barack Obama, siblingOf, White House), is not plausible. If siblingOf relation is specified formally to have a range of Person and if Person and Building are disjoint, that leads to a logical inconsistency. Nevertheless, those granular axioms are not always present in ontologies for such reasoning. Thus, we propose that Linked Data profiling that was discussed in Chapter 5 and RDF Shape Induction that was discussed in Chapter 6 can be used for understanding logical inconsistencies even when there are no explicit ontological axioms that show or detect such inconsistencies. Data profiling and RDF Shapes can be used to identify common patterns in data that can provide heuristics of inconsistencies. Once a triple is identified as inconsistent it can be directly classified as noise because it contradicts with the domain that it represents.

Plausible triples are the ones that do not contradict the domain that they represent. Plausible triples are not necessarily true but it is possible that they could be true. Plausible triples can be either Generic or Factual. 
Generic triples do not mention specific entities but rather generic concepts. For example, (family, residesIn, New York). In a generic triple, it is possible that only the subject is generic, only the object is generic, or both the subject and the object are generic. We can classify the generic triples as either true, false, or true in the past. For example, (actor, starsIN, New York)

Generally generic triples have less information value for most practical use cases. Nevertheless, if both entities are generic and if the statement is true, (e.g., (family, residesIn, city) such triples can provide schema-level information. For example, they could be used for automatically learning ontologies from textual copra.

Finally, factually false triples are the ones that contain incorrect information. For example, (Boston, capitalOf, USA) is factually false.

\subsubsection{Generation of RDF Data}

Generally, most information extraction tools extract facts as a set of triples of labels and do not transform them into RDF triples. Thus, an important preparation step of the proposed approach is to transform such textual triples into RDF triples. Input to this process is a set of string triples which is the output from the information extraction tool that extract those triples from the natural language documents. In addition to the subject, object, and predicate, most tools also extract the type of each entity in the subject and the object as shown in the \#0 in the Listing 7.1.

As the first step, an intermediate RDF representation is created where the triple is represented using the RDF model by assigning each entity an identifier and by using predefined relation and class mappings according to the schema defined by the information extraction tool as shown in 1. In this case, the type Place in the tool schema is mapped to dbo:Place class in the DBpedia ontology. In the second step, the entities are mutually disambiguated and linked to external knowledge bases. In this example, we have linked them to the DBpedia. For instance, this can be done by using a disambiguation service such as DBpedia Spotlight [156] by passing the initial triple as a sentence, i.e., "California locatedAt USA". Such services perform named entity recognition, disambiguation, and linking and provide linked entities with a confidence score. This information is used to link and establish equivalence relations to entities in DBpedia as shown in \#2. As we will be using external knowledge bases for finding evidences of the factual correctness of a given triple, we will collect other equivalence relations using services such as sameAs.org [97] as shown in \#3. With this step, we can expand the owl:sameAs links to other external knowledge bases that would be useful in the fact validation process.

Finally, in the last step the relation locatedAt is mapped to an external predicate as shown in \#4. This is not performed as a predefined mapping because the granularity of the relations may vary in the information extraction tool schema and the ontology 
of the external knowledge base. A relation mapping is one-to-one if both relations in information extraction and the properties in the knowledge base have the same level of granularity, for example, sibling Of to dbo:sibling. Otherwise, the mapping is conditional based on the domain and the range, for example, locatedAt maps to dbo:country with an instance of dbo:City and instance of $d b o$ :Country while in other cases it could map to dbo:location. This is discussed further in the inconsistency detection algorithm.

\#\# prefixes

@prefix rdfs: <http://www.w3.org/2000/01/rdf-schema\#> .

@prefix owl: <http://www.w3.org/2002/07/owl\#> .

@prefix rel: <http://loupe.linkeddata.es/semantic-lifting/rel/> .

@prefix dbo: <http://dbpedia.org/ontology/>

@prefix dbr: <http://dbpedia.org/resource/> .

\#\# 0. An example fact from information extraction tool

"California" Place "locatedAt" "USA" Place

\#\# 1. An intermediate RDF representation is created

_.1 a dbo:Place; rdfs:label "California" .

_:2 a dbo:Place; rdfs:label "USA" .

:1 rel:locatedAt _:2 .

\#\# 2. The intermediate entities are disambiguated and

\#\# linked to DBpedia

_:1 owl:sameAs dbr:Califonia .

_:2 owl:sameAs dbr:United_States .

\#\# 3. Further links are created to other knowledge bases

dbr:Califonia owl:sameAs

$<$ http://www.wikidata.org/entity/Q99>,

$<$ http://rdf.freebase.com/ns/m.01n7q $>$,

$<$ http://yago-knowledge.org/resource/California $>$,

$<$ http://sws.geonames.org/5332921/>,

$<$ http://data.nytimes.com/56975960096322330571>,

$<$ http://sw.cyc.com/concept/Mx4rvVj2upwpEbGdrcN5Y29ycA> .

dbr:United_States owl:sameAs

$<$ http://www.wikidata.org/entity/Q30>,

$<$ http://rdf.freebase.com/ns/m.09c7w0 $>$,

$<$ http://yago-knowledge.org/resource/United_States $>$,

$<$ http://sws.geonames.org/6252001/>,

$<$ http://data.nytimes.com/N84789043836346105961>, 
$<$ http://sw.cyc.com/concept/Mx4rvVikKpwpEbGdrcN5Y29ycA > .

\#\# 4. Relation mapping

rel:locatedAt $->$ dbo:country

dbr:Califonia dbo:country dbr:United_States .

Listing 7.1: Generation of RDF data

\subsubsection{Noise Detection Method}

We propose to detect and filter inconsistent and generic triples from the information extraction output before checking for factual correctness as illustrated in Figure 7.7. In this section, we describe preliminary approaches for detecting inconsistent triples and generic triples using the knowledge in external datasets such as DBpedia.

Inconsistent triple checking is performed by mapping both entities as well as relations to external datasets and considering both RDF Shapes automatically extracted from data as discussed in Chapter 6 and using the ontological axioms that define formal conceptualisations (e.g., domain and range of properties or disjoint types). The inconsistent triple detection process is defined in Algorithm 1.

Generic triple detection is performed using part-of-speech tagging using NLP tools. We use the intuition that when the subject or the object is not a proper noun, it typically refers to a generic entity rather than a specific one. If either the subject or the object is generic, the triple becomes a generic triple as illustrated in Algorithm 2. For detecting factually true triples and filtering factually false ones, we use a similar approach to fact checking by looking for evidences that confirm the given triple in an external dataset using entity disambiguation and relation mapping described in Algorithm 1.

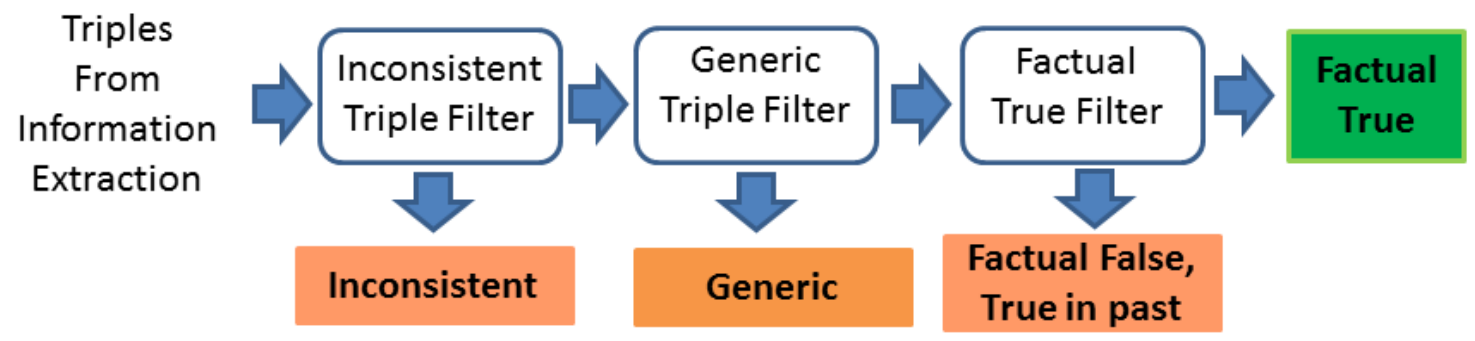

Figure 7.7: Noise detection workflow 


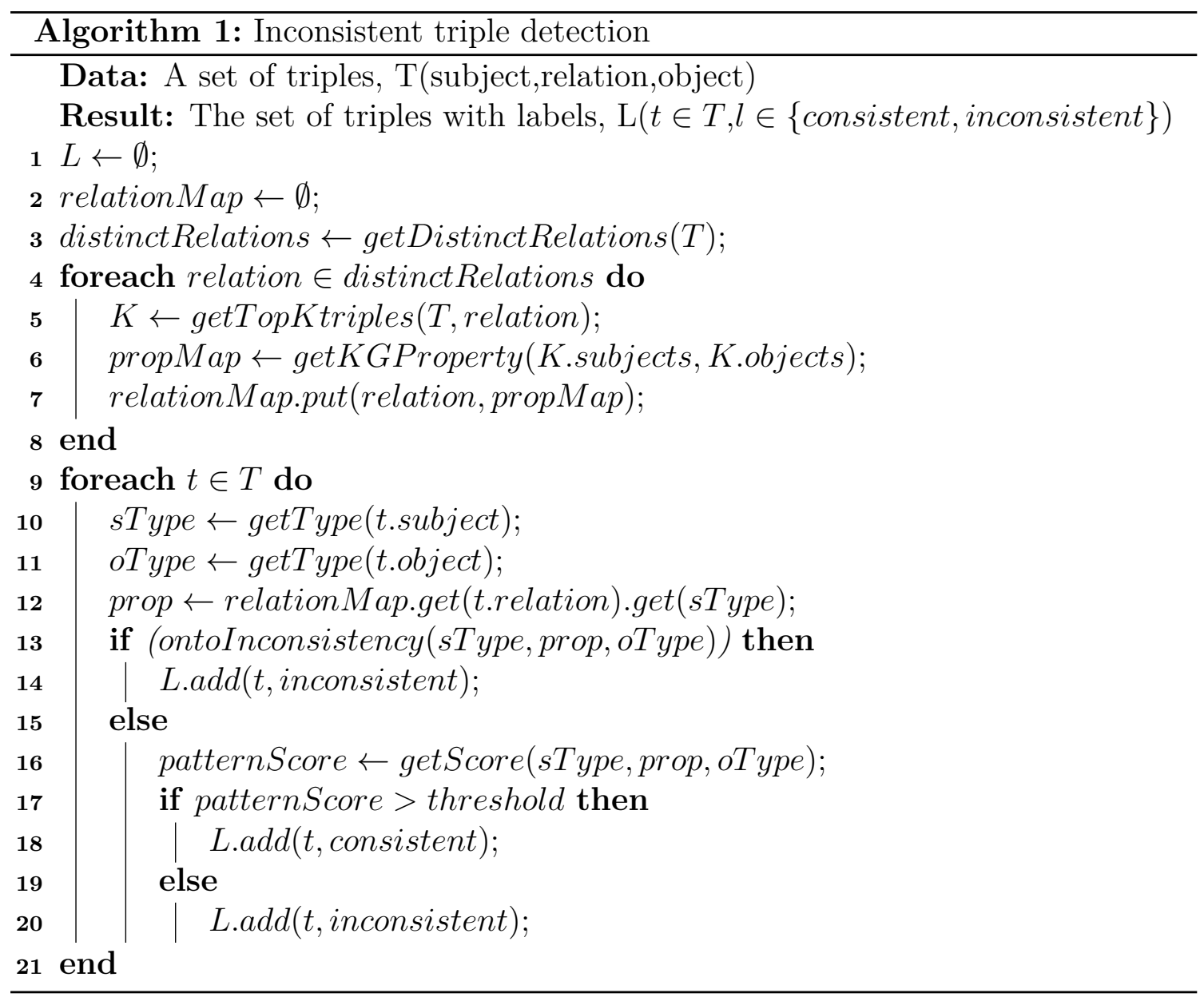

\subsection{A Method for Repairing Links in Data}

\subsubsection{Use Case}

The previous two use cases were focused on Linked Data generation, the first one on detecting quality issues of mappings and the second one on detecting noise in the output of information extraction tools. The focus of this use is on how to repair the data once that has been already generated. More concretely, the focus on the method proposed in this section is on how to repair broken links in data.

As discussed before, RDF datasets are becoming core components of most modern artificial intelligence and cognitive applications. These datasets provide a representation of real-world entities and their relationships using a graph-based model. Popular knowledge graphs such as DBpedia, Wikidata, or YAGO use the RDF model for knowledge representation, usually publishing their data as Linked Data. One of the 


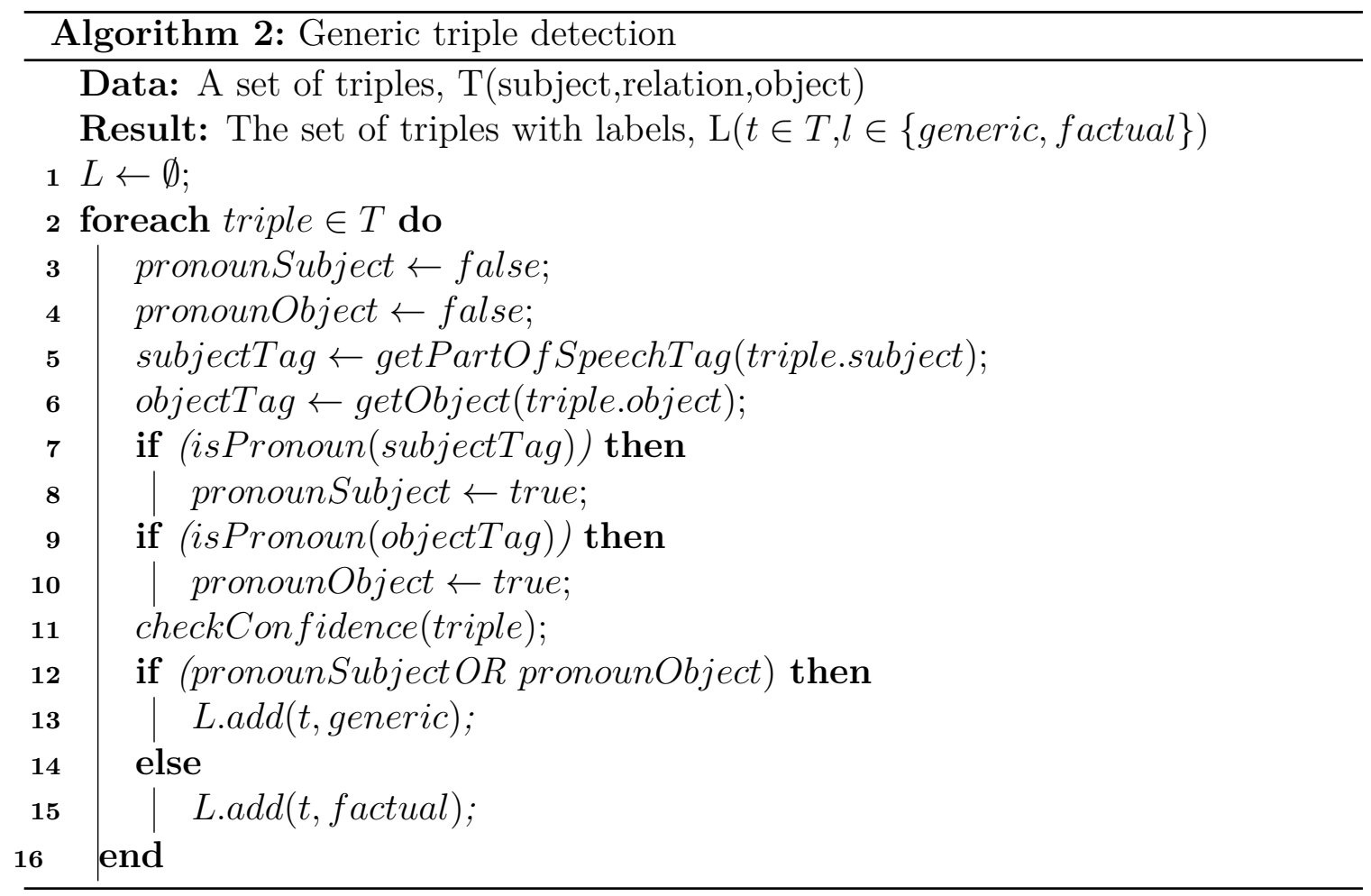

key advantages of using Linked Data is that data consumers can traverse through the typed links of the graph, to discover related entities represented by global and dereferenceable identifiers (i.e., IRIs). This provides additional value, allowing access to resources inside and outside the graph; for example, by connecting to other external datasets in the Linked Open Data Cloud. This characteristic is in the very core of Linked Data, as stated in the first and the fourth principles: "Use URIs as names for things" and "Include links to other URIs, so that they can discover more things"

On the contrary, when literal objects are used, those objects become leaf nodes in the graph, without outgoing links to further vertices. There are many situations in which it is legitimate to use literal objects, for example, when representing numerical values or dates, with clear and proper semantics associated with them. Nevertheless, we have noticed that, in practice, in knowledge bases such as DBpedia or data embedded in web pages (Web Data Commons), literals are often used to represent entities when an entity identifier (i.e., IRI), should have been used. This issue is exemplified in Figure 7.8 where the RDF graph has (a) the literal "Apple" related to two entities, and (b) the same literal is correctly disambiguated and linked to the two corresponding entities in the knowledge graph.

One problem in using strings for representing entities is that it hinders connectivity

\footnotetext{
${ }^{4}$ https : //WWw.w3.org/DesignIssues/LinkedData.html
} 


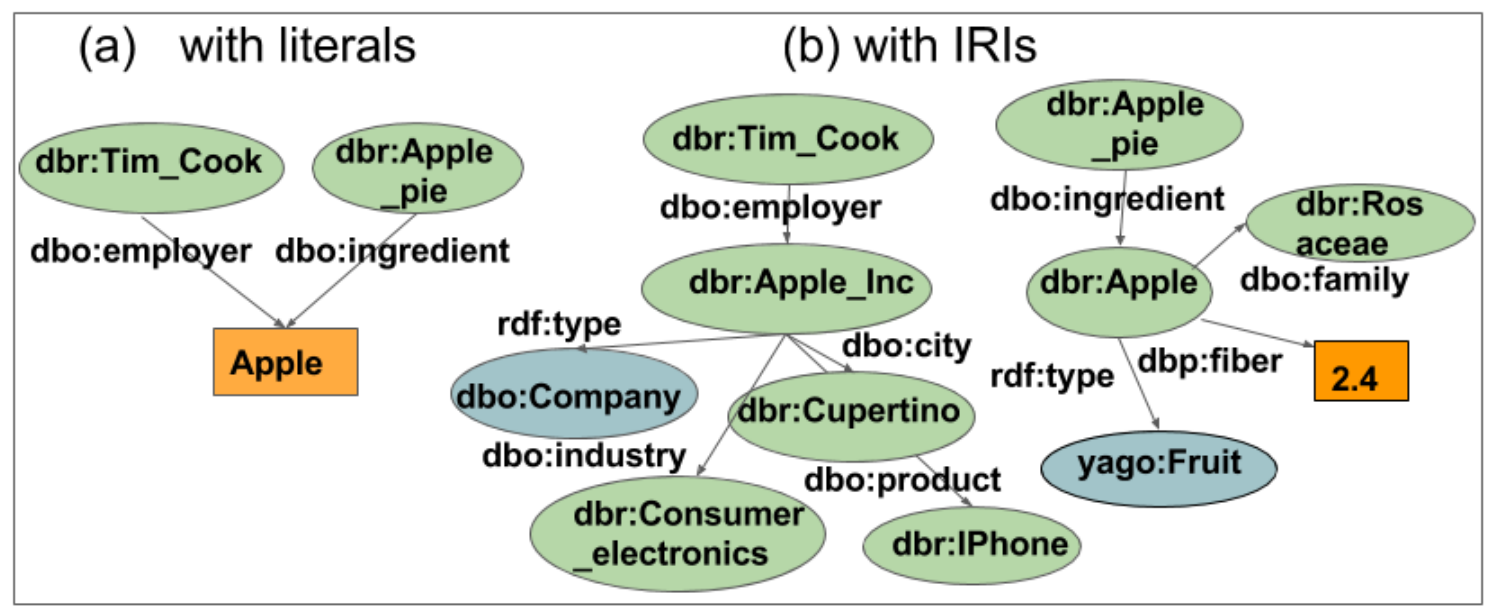

Figure 7.8: RDF graphs with (a) literals and (b) IRIs

in the graph. Even though literals are not truly isolated nodes in the graph, due to their incoming links, they are much less connected compared to those defining entities, which can have both incoming and outgoing links. Also it is not possible to provide additional information about such literals, because, unlike IRI resources, properties can not be associated with them. Ideally these issues would never happen, specially when a domain model (i.e., vocabulary or ontology) is available to define how knowledge should be represented, ensuring that proper entities and links between them are defined during the dataset construction phase. However, as we demonstrate later in this section, we find a large number of situations where this is not the case. The goal of this work is to identify such string literals and replace them with their proper entity IRIs. This will make the data more consistent, easier to query, and will increase the connectivity of the graph.

Besides connectivity, using literals where IRIs should be, introduces ambiguity of terms in the knowledge graph, similar to what happens in natural language. Coming back to the example depicted in Figure 7.8, in (Apple Pie, ingredient, Apple) and (Tim Cook, employer, Apple), the term "Apple" refers to two different real world entities. Semantic Web mitigates this problem by naming each of those entities with a distinct identifier. For instance, in DBpedia those entities are assigned dbr:Apple and $d b r$ :Apple_Inc. respectively. Thus, instead of using literals, if IRIs are used, such ambiguities could be eliminated.

Entities may be materialised as strings in knowledge graphs for several reasons. It could be due to limitations in the graph generation process. For instance, in the DBpedia extraction framework [147], when a key in a Wikipedia infobox ${ }^{5}$ does not have a mapping and the infobox value is a string, the system does not have

\footnotetext{
${ }^{5}$ https://en.wikipedia.org/wiki/Help:Infobox
} 
enough information to decide whether that string refers to an entity in the dataset or not. However, such information can be inferred later on using profiling on all the values of a given property, as we discuss later in this section. Further, in publishing structured data from relational databases or CSV as Linked Data, it is considerably much easier for the publishers to directly represent column values as strings rather than disambiguating and properly linking them. Nevertheless, such strings reduce the quality of the published data. In this work, we propose an approach for detecting string literals denoting entities and transforming them into IRIs in the graph.

This transformation might not always be possible, for example, when the detected entity does not yet exist in the knowledge graph, making it not possible to find the corresponding identifier. Even though new entities could be added to the dataset to remedy this, it is out of the scope of this method presented in this thesis. Our approach focuses on linking already existing entities, based on the detection of properties that link entities on the dataset. Hereafter, we will use "entity relation" to refer to such links. These entity relations link entities to other entities, irrespective of whether the entity is represented as an IRI or as a string literal denoting entity (e.g., "Los Angeles" or "Apple"). However, we do not consider the links to literals such as integers, dates or strings that do not denote entities (e.g., an abstract or a comment) as entity relations. Figure 7.9 illustrates an example of an entity relation between a person and a company and an example of a non-entity relation between a company and its description.

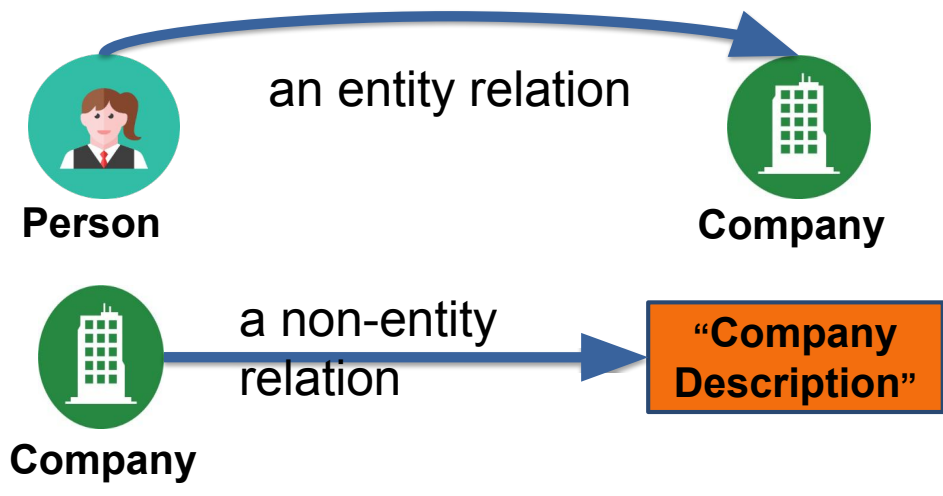

Figure 7.9: Examples of an entity relation and a non-entity relation 


\subsubsection{Approach}

The objective of the method proposed in this section is to a link repair method that can identify string literals in an RDF dataset that should be represented using IRIs. Such conversion will increase the connectivity of the RDF graph and will help to get the most benefits of the Linked Data principles. Figure 7.10 illustrates the general approach followed by the link repair method.

As discussed in the previous section, our approach is based on first identifying entity relations and then using that information to identify string candidates that can be converted into their corresponding IRIs. Thus, the proposed link repair method consists of two main steps: (a) identification of string entity relations, and (b) string literal to IRI conversion. The identification of entity relations is performed using the information from Linked Data profiling metrics in addition to the explicit information provided by ontologies. All such information is used to develop an algorithm that can distinguish entity relations from non-entity relations. String literal to IRI conversion uses information from RDF Shapes to more accurately disambiguate the string literals and find their corresponding IRI.

One the entity relations are identified, candidate string literals are selected and converted into their corresponding IRIs, the RDF dataset is modified by replacing those string literals with their corresponding IRIs. This will result in an improved RDF dataset that contains an RDF graph with better connectivity and less ambiguity.

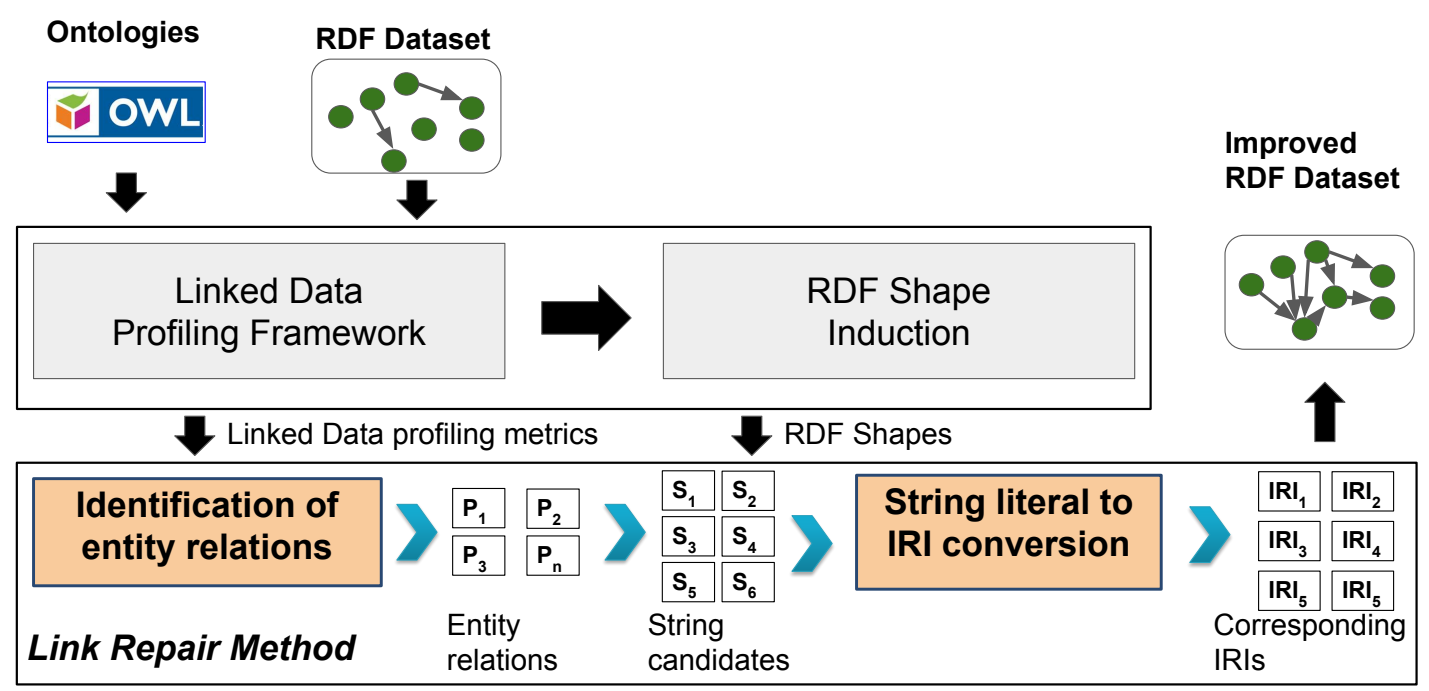

Figure 7.10: Link repair method workflow 


\subsubsection{Things as Strings}

The main problem that the proposed method plans to address is that how to identify the presence of string literals that denote things. To this end, the following research questions are defined:

- How to identify string literals that denote entities in a dataset?

- How to transform those string literals into IRIs that correspond to entities they denote?

- How to measure the improvement in quality because of the transformation?

The first hypothesis of this work is that string literals that denote entities can be identified by detecting the relations that link entities (i.e., entity relations). Some entity relationships can be defined explicitly in the ontology. For example, OWL ontology properties are explicitly defined as either object properties or datatype properties. All object properties are entity relations by definition. For example, the dbo:capital property in Listing 7.2 defines an explicit entity relation between a Country and a City. This property should not have string literals as objects in a triple, since that is not consistent with the schema and should therefore be corrected. RDFS properties do not make this explicit distinction; an RDFS property can have both entities and literals as objects of a triple. Thus, it is not straightforward to detect entity relations in RDFS properties. For example, the dc:creator property in Listing 7.2, which explicitly defines the range to be an Agent, is an entity relationship but it is not possible to identify that just using the schema. In contrast, if the range is either rdfs:Literal or an instance of rdfs:Datatype, we can assume that such property is not an entity relation.

\footnotetext{
@prefix rdf: <http://www.w3.org/1999/02/22-rdf-syntax-ns\#> .

@prefix rdfs: <http://www.w3.org/2000/01/rdf-schema\#> .

@prefix owl: <http://www.w3.org/2002/07/owl\#> .

@prefix dbo: <http://dbpedia.org/ontology/> .

@ prefix dbp: <http://dbpedia.org/property/> .

@prefix dc: <http://purl.org/dc/terms/> .

\# (1) dbo:capital property definition

dbo:capital a owl:ObjectProperty;

rdfs:domain dbo:Country;

rdfs:range dbo:City .

\# (2) dc:creator property definition

dc:creator a rdf:Property ;
} 
rdfs:range dc:Agent .

\# (3) dbp:birthPlace property definition

dbp:birthPlace a rdf:Property .

\section{Listing 7.2: Property definition examples}

RDFS properties that do not constraint the range can potentially have both rdfs:Resource and rdfs:Literal objects; for example, dbp:birthPlace in the DBpedia dataset. Even though intuitively we can guess that birthPlace is an entity relationship, its schema does not provide such information. Thus, we propose an approach for detecting whether a property is a relation between two entities, even when the schema information is not available using data profiling.

Once entity relations are identified, either using explicit ontological axioms or using data profiling, the goal of our approach is to use proper entity identifiers (IRIs) in such relations instead of literals, introducing new links, which improves the connectivity of the graph and makes the data easier to query.

Following this line of thought, the second hypothesis of this work is that the connectivity of a knowledge graph can be improved by transforming literal nodes in entity relations into their corresponding entity IRIs. It is worth to note that not all RDF graphs are mathematical graphs. In RDF graphs, edges can also act as nodes (e.g., property definitions). As for this work, when calculating connectivity, we work at the data level (ABox), not at the model (TBox), thus not facing this issue.

When we convert literals into IRIs we are introducing new links, thus increasing the connectivity of the graph, which would enhance the quality of query results, allowing to traverse to more entities. As a critical example, if an RDF graph has two connected graph components ${ }^{6}$, by adding one of these conversions we could link the two components that would become a single component with all nodes. This would lead to much more results in a query and improve how navigable the graph is.

Some problems similar to the one we target in this section exist, and some solutions for them have been proposed. Therefore in this section we clarify how our goal and approach differ from them.

Named entity disambiguation Named entity disambiguation generally refers to the identification of entity mentions (referred to as surface forms) in natural language text and linking them to a corresponding entity in a knowledge graph such as DBpedia or YAGO. This is a challenging task due to two main concerns: ambiguity and synonyms. Ambiguity is when a single surface form can refer to multiple entities, for example, "Java" can refer to the programming language or to the Indonesian

\footnotetext{
${ }^{6}$ We use component as defined by graph theory, describing a subgraph in which all nodes are connected but which is not connected to other subgraphs in the supergraph.
} 
island. Synonyms are when the same entity is referred from multiple surface forms, for example, "Tim Berners-Lee" and "inventor of the Web" refers to the same entity. The objective of this work is quite related to named entity disambiguation; however, in our case the literals are already in a knowledge graph and a lot of structural information is already available. Nevertheless, textual information such as surrounding words is not available. As a consequence, our approach aims to focus more on structural features and compensate for the lack of textual information using the entity it is connected to.

Web Table Annotation Web table annotation refers to semantically annotating relational HTML tables extracted from the Web. Generally this consists of three sub-tasks: (a) table-to-class matching in which each table is mapped to a class in an ontology, (b) attribute-to-property matching in which each class-column relation is mapped to a property in an ontology, and (c) row-to-instance matching in which each

row is mapped to an instance of the dataset. In the row-to-instance task, a set of row labels (a column that uniquely identifies the row) are mapped to corresponding entities in a knowledge graph. This task is related to ours because Web Tables also have a more structured input and the set of literals to be converted into entities are already grouped together into similar ones. Thus, in our approach we use some techniques inspired by table annotation algorithms such as class matching and iterative annotation [203].

\subsubsection{Link Reparation Technique in Data}

This section outlines our approach to detect string literals that denote entities which, thus, can be potentially converted to IRIs. This approach is based on the intuition that such strings can be detected by first identifying the aforementioned entity relations (links that relate entities).

\section{Identification of entity relations}

We follow a two-fold approach. On the one hand, we follow an ontology-based approach by checking whether the definition of the RDF property is available. When a rich ontology is available, entity relations can be directly identified. By definition, OWL object properties are entity relations. RDFS properties with either rdfs:Literal or an instance of rdfs:Datatype as range are not entity relations.

On the other hand, when not enough information is available, we follow a datadriven approach, exploring the characteristics of the given property in the dataset. For example, in the DBpedia dataset, there are more than 50,000 properties that are only defined as RDFS properties, without any further schema information. Because their lack of semantics, we identify entity relations by analyzing class-property 
combinations, rather than properties independently (e.g., dbo:Plant-dbp:family vs only dbp:family).

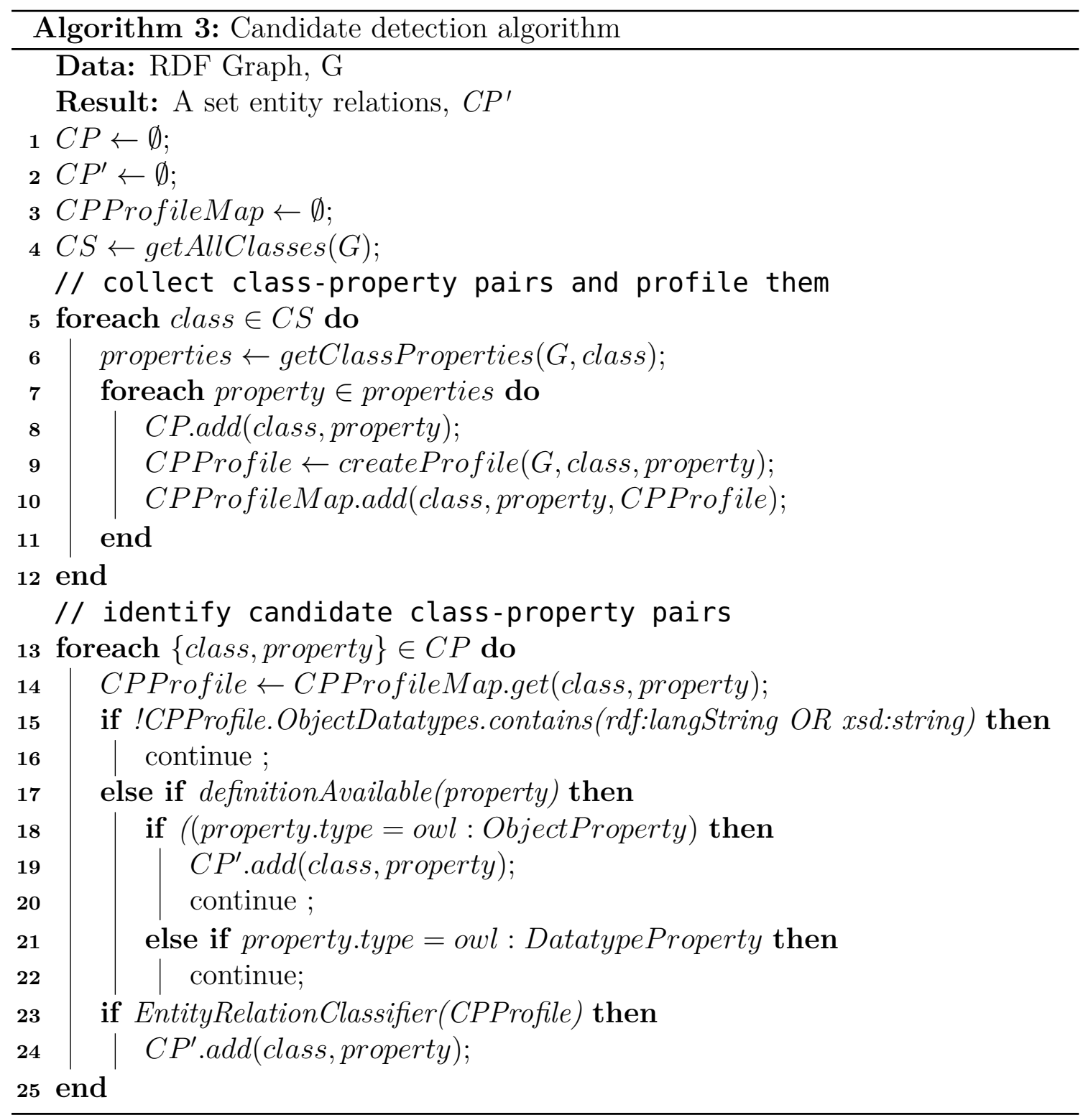

The use of class-property combination allows us to analyse properties at a more granular level. The algorithm for identifying entity relations in class-property combinations of a given RDF graph is outlined in Algorithm 1. First, we generate all class-property combinations in the RDF graph (L5-8) and use the Linked Data profiling framework mentioned in Chapter 5 to generate Class-Property Profiles. Table 7.7 illustrates the information extracted during profiling, and Listing 7.4 shows 
an example of it. Then, we analyze each class-property pair to identify if they represent an entity relationship or not, using both their definitions as well as the generated Class-Property Profile. These profiles are used to identify entity relations. We only analyze the ones that have at least one string literal (L15), because our goal is to convert strings into IRIs. We first try to decide based on the property definition if available (L17-22); if not, they are passed to a classifier trained on a set of manually labeled data. The classifier takes the elements of the Class-Property Profile as features and performs a binary classification to identify entity relations.

\section{String literal to IRI conversion}

Once the entity relations are identified, the next step is to convert their literal objects to entity IRIs. The goal of this step is to increase the quality of the dataset by improving the connectivity and also making the graph consistent with the property semantics (i.e., no violations of range constraints). For this step, the input is the object values of a given class-property pair. These values can contain both IRIs and string literals (see, Listing 7.3).

We use a standard disambiguation tool, DBpedia Spotlight [156], which provides language-specific models; for example, with the Spanish data, we use the Spanish model. As our use case is not the typical use case of disambiguation for text annotation, we propose several approaches for making the input suitable for disambiguation. Further, we also aim to make the best use of entities that are already disambiguated to mutually disambiguate the remaining strings.

dbr:Hulk_Hogan dbp:birthPlace "Georgia, USA"@en .

dbr:Miriam_Blasco dbp:birthPlace "Valladolid, Spain"@en .

dbr:Sidney_Green dbp:birthPlace "Brooklyn"@en .

dbr:Michael_Jordan dbp:birthPlace dbr:Brooklyn .

dbr:Raul_(footballer) dbp:birthPlace dbr:Madrid .

Listing 7.3: A sample of dbo:Athlete/dbp:birthPlace values

@prefix loupe: <http://ont-loupe.linkeddata.es/def/core/ $>$.

@prefix dbo: <http://dbpedia.org/ontology/> .

@ prefix dbp: <http://dbpedia.org/property/> .

@ prefix rdf: <http://www.w3.org/1999/02/22-rdf-syntax-ns\#>

cpp:en-dbp-201610-1 a loupe:ClassPropertyProfile ;

loupe:aboutClass dbo:Person ;

loupe:aboutProperty dbp:birthPlace ;

loupe:instanceCount "697783" ^ xsd:long ; 


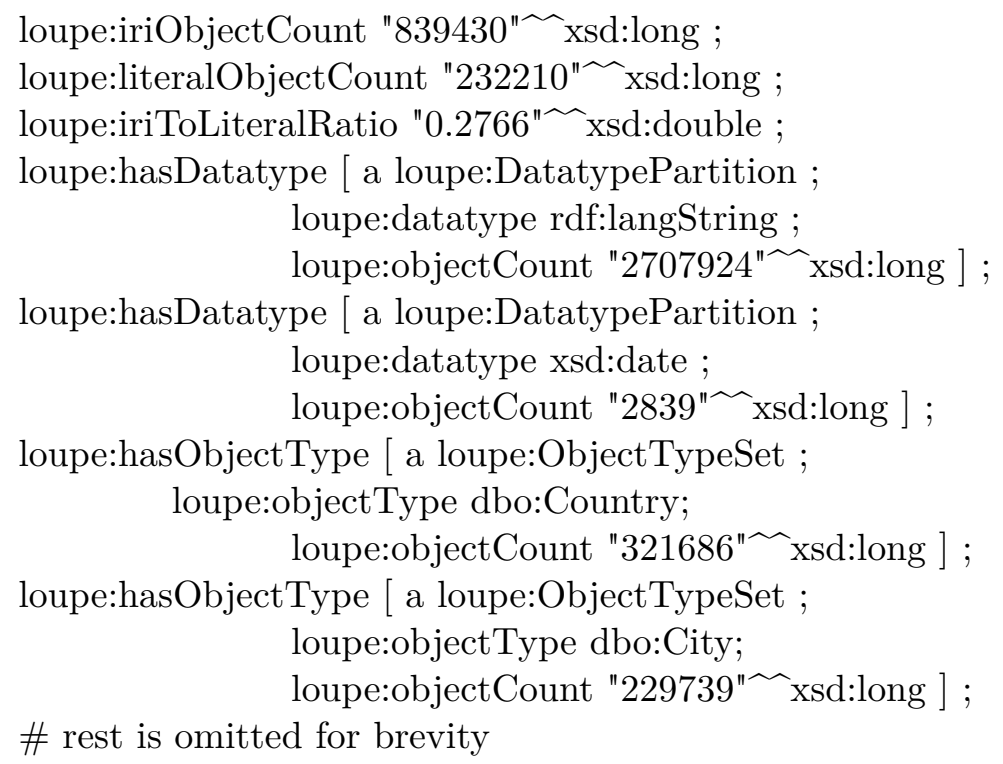

Listing 7.4: A snippet from a class-property profile

Table 7.7: Class-property profile definition

\begin{tabular}{|l|l|}
\hline Property & Description \\
\hline aboutClass & The class of the class-property profile. \\
\hline aboutProperty & The property of the class-property profile. \\
\hline instanceCount & $\begin{array}{l}\text { The number of instances of the given class that have at } \\
\text { least one triple with the given property. }\end{array}$ \\
\hline objectCount & $\begin{array}{l}\text { The number of objects with this class-property combina- } \\
\text { tion. This also equals to the number of triples containing } \\
\text { this class-property combination. }\end{array}$ \\
\hline distinctObjectCount & $\begin{array}{l}\text { The number of distinct objects with this class-property } \\
\text { combination. }\end{array}$ \\
\hline iriObjectCount & The number of IRI objects. \\
\hline literalObjectCount & The number of literal objects. \\
\hline hasObjectType & The distribution of types of IRI objects. \\
\hline hasDatatype & The distribution of datatypes of literal objects. \\
\hline
\end{tabular}

Generating context Disambiguation tools need textual content for them to have sufficient context to disambiguate a string (i.e., the surface form of an entity) and link it to the corresponding entity. Thus, we generate a textual context for each string by utilizing the entity that is directly connected to the string in the knowledge graph. First, we create a simple sentence by combining the entity label (as subject), the 
property label (as predicate), and the string (as object). Then, we complement it by appending the description of the entity to it, generating a complete context, as shown in Listings 4 and 5 , in which the first line is the generated sentence and the remaining is the description extracted from the dataset (using textual descriptions from properties such as dbo:abstract, rdfs:comment, or dc:description). Our experiments show that the generated context is effective in disambiguating entity strings.

Tim Cook employer Apple.

Timothy Donald "Tim" Cook is an American business executive, industrial engineer and developer. Cook is the current and seventh Chief Executive Officer of Apple Inc., previously serving as the company's Chief Operating Officer, under its ....

Listing 7.5: Tim Cook and Apple

Apple Pie ingredient Apple.

An apple pie is a fruit pie, in which the principal filling ingredient is apple. It is, on occasion, served with whipped cream or ice cream on top, or alongside cheddar cheese. The pastry is generally used top-and-bottom ...

Listing 7.6: Apple Pie and Apple

Type identification In contrast to text disambiguation from unstructured sources, our use case can rely on the fact that we are aiming to disambiguate entities of the same (or similar) types which are already known. This is because all the strings are taken from a single entity relation. For example, in the "employer" relation, we expect all the objects to be of the type Company or Organization.

Thus, we aim to predict the range of the entity relation and use it to guide the disambiguation or to choose the correct entity when the disambiguation results in multiple entities.

On the one hand, when there is a large number of existing IRIs, we can analyze the frequencies of types of those IRIs to derive heuristics about the entity type that a given set of strings denote. Based on this, we can perform disambiguation taking into account the most frequent $\mathrm{K}$ types that we observe in the existing IRIs.

On the other hand, when the IRI percentage is low, we can not reliably do a top $\mathrm{K}$ frequency analysis. In this case, we follow an iterative approach. We perform disambiguation in the first iteration and resolve all strings that are disambiguated with high confidence. Then, we perform the type analysis of those entities to predict the range of the relation. Then, in the second round of disambiguation, we use that information for selecting the correct entity when the disambiguation confidence is low or when the disambiguation tool gives multiple entities. This technique was inspired by Web Table annotation algorithms [203]. 
Confidence calculation and string replacement As the final step, we calculate confidence values based on the confidence returned by the disambiguation tools and the most frequent $\mathrm{K}$ types identified during the type identification phase.

When the confidence is higher than a predefined threshold, we replace the string with the corresponding entity IRI in the knowledge graph. When the confidence is low, we keep the string literal as it is in the graph.

\subsubsection{Identification of Candidate Properties}

Based on the techniques discussed in the previous section, we have developed a method for analysing the structure of datasets to decide which strings denote entities, and replace them by the corresponding IRIs. We have used it to check the presence of string literals denoting entities in two large datasets.

Table 7.8: Top entity relations with strings in LOD Cache

\begin{tabular}{|l|l|l|l|l|}
\hline Property & \# of lit. & \# obj. & \% lit. & $\begin{array}{l}\% \text { of } \\
\text { IRI }\end{array}$ \\
\hline dct:language & $24,942,739$ & $24,943,294$ & 100 & 0 \\
\hline rdfs:seeAlso & 83,156 & $1,205,624,455$ & 00.01 & 99.99 \\
\hline rdfs:isDefinedBy & 13,112 & $172,312,105$ & 00.01 & 99.99 \\
\hline dct:license & 10,080 & 287,750 & 3.50 & 96.50 \\
\hline dct:publisher & 4,312 & 49,312 & 8.74 & 91.26 \\
\hline
\end{tabular}

To identify entity relations with string objects, first we extracted all explicit entity relation properties (according to their definition) from the Linked Open Vocabularies $(L O V)$ catalogue. These properties should not have any literal objects. Then we analysed two large datasets: (a) LOD Cache and (b) Web Data Commons (WDS) $J S O N-L D$ embedded data to identify triples where such properties had string objects.

The results are shown in Table 7.8 and Table 7.9, which show, for each property, the number of literals, the total number of objects, and the corresponding literal and IRI percentages. 
Table 7.9: Top entity relations with strings in WDC

\begin{tabular}{|l|r|r|r|r|}
\hline Property & \# of lit. & \# of obj. & \% lit. & \% IRI \\
\hline schema:creator & $15,782,874$ & $16,573,426$ & 95.23 & 4.77 \\
\hline schema:brand & $2,420,908$ & $4,694,411$ & 51.57 & 48.43 \\
\hline schema:author & $1,500,898$ & $26,193,682$ & 5.73 & 94.37 \\
\hline $\begin{array}{l}\text { schema: } \\
\text { hiringOrganization }\end{array}$ & $1,231,840$ & $1,251,870$ & 98.84 & 1.16 \\
\hline schema:publisher & 560,577 & $31,317,151$ & 1.79 & 98.21 \\
\hline
\end{tabular}

For identifying entity relations that do not have a rich property definition (i.e., RDFS properties without any constraint), we applied the algorithm introduced in Section 6.2 to the Spanish DBpedia dataset. We could identify 25,560 class-property combinations that are entity relations containing string literal objects. There were $23,880,424$ strings as objects of those properties. By analyzing the corresponding object types (after converting to IRIs and linking them to existing entities) of each class-property relations, the most common types of entities that are denoted by strings are shown in Table 7.10.

Table 7.10: Top types of entities as strings in DBpedia

\begin{tabular}{|l|r|}
\hline Type & \# of strings \\
\hline skos:Concept & $7,691,836$ \\
\hline dbo:Person & $6,186,125$ \\
\hline dbo:Place & $4,904,153$ \\
\hline dbo:Organisation & $3,267,411$ \\
\hline dbo:Work & $2,319,624$ \\
\hline
\end{tabular}

\subsubsection{Literal to IRI Conversion}

For testing the literal to IRI conversion phase, we selected a subset of entity relations from the Spanish DBpedia data we identified in the previous step. This subset includes all class-property relations of the classes: dbo:Athlete, dbo:SportsTeam, and dbo:SportsEvent. Table 7.11 shows the results of this conversion process. 
Table 7.11: Literal to IRI conversion

\begin{tabular}{|l|r|r|r|r|}
\hline \multirow{2}{*}{ Class } & \multirow{2}{*}{ \# of props } & \multirow{2}{*}{ \# of strings } & \multicolumn{2}{|c|}{ converted to IRI } \\
\cline { 4 - 5 } & & & \# & \% \\
\hline dbo:Athete & 497 & 376,970 & 154,854 & $41.01 \%$ \\
\hline dbo:SportsEvent & 367 & 350,105 & 121,254 & $34.63 \%$ \\
\hline dbo:SportsTeam & 220 & 169,647 & 98,488 & $58.05 \%$ \\
\hline
\end{tabular}

The low recall is mainly due to the large number of real-world entities that do not have a corresponding page in Wikipedia. In such cases, it is not possible to find an IRI. For example, the string "Kellyn Huehn" denotes an MMA fighter in Indiana but because she is not in Wikipedia, the string is not disambiguated. We will look into generating new identifiers for such entities as future work.

\subsection{Type Prediction using Binary Classifiers}

\subsubsection{Use Case}

In the Semantic Web vision paper [30], Berners-Lee et al. identify the value of having access to structured collections of information with their associated explicit semantics and inference rules that can be used for automated reasoning. Semantic Web has come a long way since then and a large number of Semantic Web standards have been developed and a massive volume of data is available as a part of the Linked (Open) Cloud. Such data is generally expressed using ontologies or vocabularies and is represented using the RDF model.

Naming things using globally identifiable URIs and providing their type information plays an essential role in making the data on the Semantic Web discoverable and reusable. For instance, such information allows a dataset to answer the query, "What is La Tomatina?" by looking at its type declaration. Further, such information enables exploratory queries such as "Find all scientists in a given knowledge graph". For instance, if a knowledge base contains a type declaration, e.g., Albert Einstein is a Scientist, this will allow him to be included in the aforementioned query. Nevertheless, type information is not always available for all instances in a dataset due to various reasons such as deficiencies in the data generation or transformation process (e.g., lack of mappings) or deficiencies in source data (e.g., missing type information).

In the Linked Open Data cloud, DBpedia [17] stands out as the central hub of Linked Open Data (LOD) because it provides a vast amount of information and most other datasets in the LOD cloud link to DBpedia. DBpedia data is extracted from Wikipedia and transformed to RDF using the mappings. While being a useful source of information containing more than 6.6 million entities (English DBpedia, 2016-10 
version), DBpedia also contains a large number of entities without type information (or only typed as owl:Thing which does not add much information value). Specifically, for the aforementioned DBpedia, 1.1 million entities do not have a type.

To this end, the objective of this work is to propose a data-driven type prediction approach that predicts the type of a given instance using its structural information in the dataset. The proposed approach this study is generic and applicable to any knowledge graph and we evaluate its feasibility and performance using a set of experiments carried out using the English DBpedia and the Spanish DBpedia datasets. We chose DBpedia for experiments as it a large and a high-relevant dataset in the Linked Data Cloud. The Spanish DBpedia is the second largest DBpedia in size, after the English DBpedia, and around $40 \%$ of its content is exclusive, that is, the information that cannot be found in the English DBpedia [164].

\subsubsection{Approach}

Figure 7.11 shows the approach we propose to predicting types using the Linked Data profiling information. We propose a machine learning based binary classifier that uses the presence of a set of properties encoded as binary features to predict if a given instance belongs to a certain class or not.

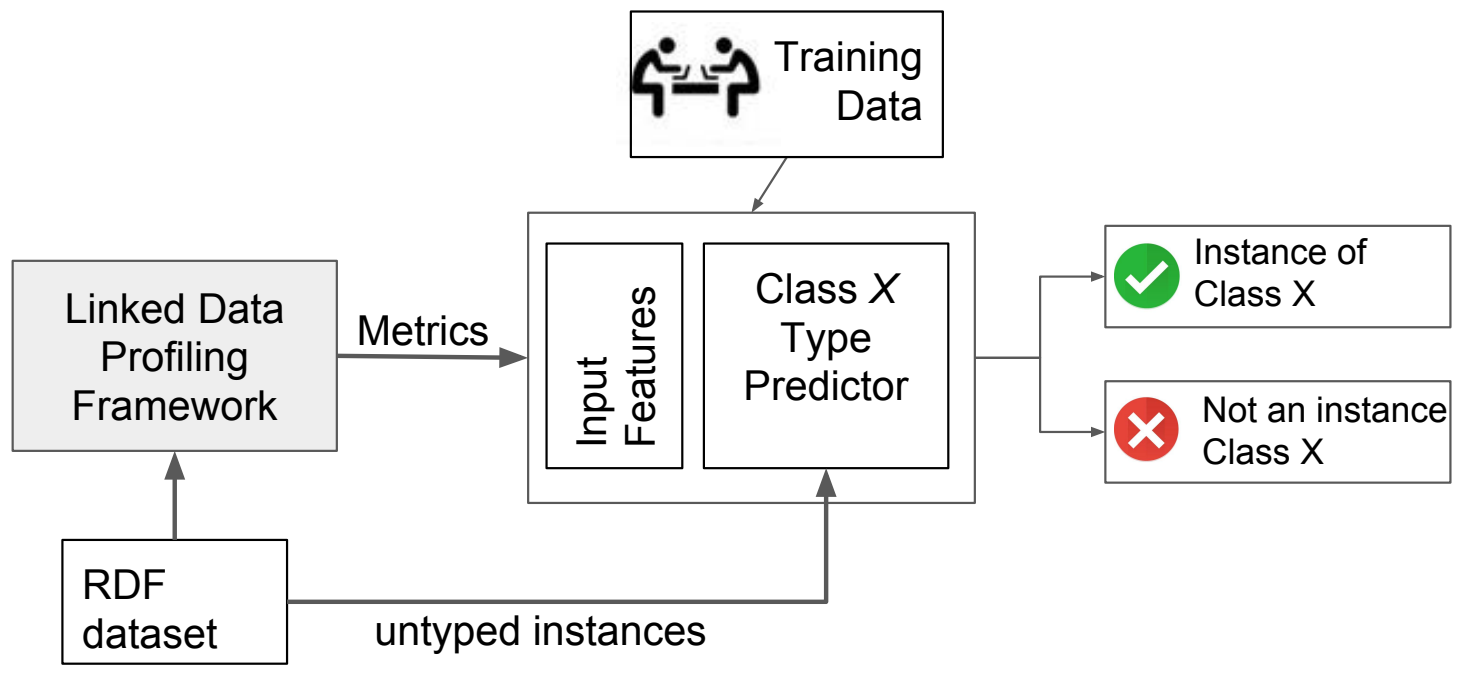

Figure 7.11: Type prediction method 


\subsubsection{Feasibility analysis}

This work is motivated by the fact that type information is not complete in datasets. The main objective of this work is to propose techniques for completing type information and evaluating such methods. One of the goals of the proposed approach is to evaluate the feasibility of different alternatives to implement a binary classifier for type prediction and also to evaluate how the proposed binary classifier performance will change depending on the complexity of the data. Thus, the main research questions that are explored while developing this method are:

- RQ1. Is it possible to build a model that can predict if a given instance belongs to a type using the properties the instance has?

- RQ2. How does the accuracy of the model change based on the diversity of the data? (e.g., the number of distinct classes)

- RQ3. Is a model trained from one dataset valid for another dataset?

The main research question, $R Q 1$, analyses the feasibility of predicting the type of an instance based on the properties it is associated with. These properties are the links that connect a given instance to other instances or literal values. Two different types of properties can be considered: the outgoing properties and incoming properties. For instance, the RDF snippet shown in Listing 7.7 contains a set of triples related to the entity dbr:Albert_Einstein. With respect to this entity, properties birthPlace, birthDate, deathPlace, deathDate, spouse, doctoralAdvisor, award, field are outgoing properties while author, notableStudent, father, spouse, influencedBy are incoming properties.

@prefix dbo: <http://dbpedia.org/ontology/> .

@prefix dbr: <http://dbpedia.org/resource/> .

@prefix xsd: <http://www.w3.org/2001/XMLSchema\#> .

dbr:Albert_Einstein dbo:birthPlace dbr:Ulm;

dbo:birthDate "1879-03-14" xsd:date;

dbo:deathPlace dbr:Princeton;

dbo:deathDate "1955-04-18" ^ $x$ xd:date;

dbo:spouse dbr:Elsa_Lowenthal;

dbo:doctoralAdvisor dbr:Alfred_Kleiner;

dbo:influencedBy dbr:Ernst_Mach;

dbo:award dbr:Nobel_Prize_in_Physics;

dbo:field dbr:Physics .

dbr:The_Evolution_of_Physics dbo:author dbr:Albert_Einstein . 
dbr:ETH_Zurich dbo:notableStudent dbr:Albert_Einstein . dbr:Hans_Albert_Einstein dbo:father dbr:Albert_Einstein . dbr:Elsa_Lowenthal dbo:spouse dbr:Albert_Einstein . dbr:Nathan_Rosen dbo:influencedBy dbr:Albert_Einstein . dbr:David_Bohm dbo:influencedBy dbr:Albert_Einstein .

\section{Listing 7.7: A snippet from an RDF graph}

As it can be seen, the same property can be considered both as an outgoing property as well as an incoming property as in the case of spouse or influencedBy. Furthermore, the same property can be used to link an entity with multiple other entities as in the case of influencedBy in this example. Thus, in this research question we analyse if it is possible to predict the type of an instance, (e.g., that Albert Einstein is a scientist) by analysing the properties that are used to link the given instance to other instances and literal values.

The objective of the second research question, $R Q 2$, is to analyse how the diversity of the data affect the type predictors. As we will discuss in the next section, we propose an approach to build binary classifier models for each class in a given ontology that will distinguish if a given instance belong to that class or not. For instance, a binary classifier for the dbo:Scientist class will classify Albert Einstein as a Scientist or true and Donald Trump as not a Scientist or false. In this question, we will explore the accuracy of the classifiers change with the diversity of the training and test data. For instance, if the data only contain instances of two distinct classes (e.g., Person and Place) that have significantly different characteristics, our intuition is that it is easier to distinguish between those two. Nevertheless, it is not as easy to identify a Scientist from a Politician as those two classes share a common super class Person and have more attributes in common. Furthermore, if the data contain a large number of different classes such as Person, Place, Event, Food, Work, or Biomolecule, our intuition is that this will make the classification problem harder and could possibly lower the performance of the classifier. Thus, we explore this research question through experimentation.

The objective of the third research question, $R Q 3$, is to analyse if it is possible to build binary classifiers for each class that will work in other datasets than the one it was trained with. If this is true, this will increase the usefulness of the model as a model trained using one dataset can be used with many other datasets for type prediction task to complete the information of other datasets.

In this context, we have formulated three hypotheses, related to the research question, that are evaluated in this paper. Those are:

- Hypothesis 1 - A Machine Learning binary classifier can be used for type prediction with the presence of properties as features with high precision. 
- Hypothesis 2 - The accuracy of the aforementioned classifier will depend on the diversity of data (i.e., the number of different types of instances in data)

- Hypothesis 3 - A model learned from one dataset can be used to predict types of another dataset. (e.g., learning from Spanish DBpedia and predicting on English DBpedia or vice versa).

In the evaluation of the proposed approach, we validate each of these hypotheses.

\subsubsection{Binary Classifiers as Type Predictors}

Several approaches have been proposed for the type prediction problem in large datasets. Most of these approaches are tested on DBpedia because of its relevance as the central hub of the Linked Data cloud.

One of the most prominent approaches in the area is the SDType [187] system, which has been applied to DBpedia both for its evaluation as well as for the dataset itself when being published. SDTypes exploits the information about the statistical distribution of properties over types to infer new ones. That is, in a nutshell, if a resource has the same properties as another one of a given type, then it can be inferred that such resource should be typed with the given type. We follow a similar line of thought in the work presented in this paper. However, unlike SDType, we use binary classifiers for each given class, compared to the multilabel classifiers in SDType.

More recent studies have proposed the use of machine learning techniques, like the one used to identify wrong mappings in DBpedia [200]. Specifically focused on improving the types of resources in a dataset, some contributions have been explored, using multilabel approaches [223, 239], varying on the algorithms applied and how the training data is selected. In general, all of them define the process of assigning types as a multilabel classification problem, as several types are expected for each resource. In our work, we formulate the type prediction as a binary classification problem by generating a model for each of the classes in the given ontology.

Taxonomy-based classification approaches for a multilabel process, which use the ontology to divide the training data into different subsets according to different criteria, has been also proposed [202]. Melo et al. [155] provide a comprehensive discussion on state of the art.

In this work, we propose an approach for predicting the type of an instance resource based on its structural characteristics, i.e., the properties it has. We formulate this type prediction problem as a binary classification problem where, for each class in the corresponding ontology (e.g., DBpedia ontology in this case). Thus, each class will have a prediction model that will predict if a given instance belongs to that class or not based on the properties a given instance resource has. For instance, we can 
build a binary classifier model for dbo:Holiday class, which predict if a given instance is a holiday or not based on the properties it has.

\subsubsection{Feature Engineering}

In the approach, each feature is binary and is associated with a given property. If the corresponding property is present in the instance, the value is 1 and 0 otherwise. The next decision is which properties to include as features. Trivially, all properties in a given ontology can be included as features. However, this will lead to sparse feature matrix with most features having all zeros because they are not related to any of the classes in our training/test data. Thus in our experiments, we limit the features to a subset of properties, i.e. all properties that are present in at least a single triple in the training data.

\subsubsection{Training}

The next step is to prepare data for training the binary classifier model of each class. For this, we need a set of positive training data as well as a set of negative training data. Positive training data can be easily generated by querying all instances of a given class. For instance, for the classifier model for dbo:Holiday class, that will be all Holiday instances. Nevertheless, negative training data generation can be done in several different ways. For instance, a class that is disjoint with Holiday can be chosen, e.g., Person, and all instances of that class can be used as negative training data. However, this will create a more simpler setting than the real-world data where only instances of the two classes are present. By adding more classes to the negative training data, we can make this setting more realistic. If the training data contains a representative sample of instances of all classes, we can assume the learned models to be representative.

Further, if the dataset contains some noisy data, a manual inspection of the selected instances is needed because some of the type information can be wrong. For example, in the DBpedia dataset there were several erroneous types in its resources. In this manual analysis, we identified a few common errors, for instance, pages with lists of people being typed as person.

In this paper, we have presented an approach for type prediction of instances based on the structural information, i.e., the properties the instance has. The experiments that were carried out demonstrate that a binary classifier model can be built with high accuracy for type prediction using structural information as features and also the accuracy of the model depends on the diversity of the training knowledge graph. Further, the experiment demonstrates that a model that is trained on one dataset can be used for type prediction in a different dataset. 
As future work, on the one hand we plan to extend this study to analyse the effects of diversity and dataset complexity by using further granular levels of classes in the ontology (such as more deeper levels of subclasses, e.g., $2^{\text {nd }}, 3^{\text {rd }}, 4^{\text {th }}$ levels) as well as testing the generated models with other multilingual datasets such as the French DBpedia, Italian DBpedia, German DBpedia, etc. On the other hand, we plan to extend this study to compare the performance of the approach proposed in this paper with other NLP-oriented approaches for type prediction to see the effectiveness of type prediction based on structural information and based on NLP features. Our intuition is that the ensemble approach that uses both structural features and NLP features will yield even better performance. 


\section{Chapter 8}

\section{Evaluation}

As discussed in Chapter 3, the main objective of this thesis is to propose novel and scalable methods for automatic quality assessment and repair of Linked Data. This main objective was further subdivided into four sub-objectives: (i) to provide means for representing quality assessment results along with all associated metadata in a comparable manner using well-established standards., (ii) identify information that could be automatically generated on large-scale datasets and could be used for quality assessment and repair tasks, (iii) to propose methods for automatically deriving Linked Data validation constraints for large datasets, and (iv) to develop methods for automatic quality assessment and repair.

Aligned with the aforementioned objectives, four main contributions are derived during this thesis:

i) LDQM, A Linked Data quality model for representing Linked Data quality in a standard manner (Chapter 4)

ii) Loupe, A framework for Linked Data profiling (Chapter 5)

iii) An automatic RDF Shape induction method using the Linked Data profiling metrics (Chapter 6), and

iv) A set of methods for automatic quality assessment and repair using Linked Data profiling metrics and RDF Shapes (Chapter 7)

In Chapter 3, Work Objectives, we have identified a set of restrictions and assumptions that defined the scope of the work and formulated 9 hypotheses that we will use to evaluate the aforementioned contributions (Section 3.4). Furthermore, we designed evaluation plans for each of the hypotheses (Section 3.5).

In this chapter, we evaluate the hypotheses related to each of the contributions according to the evaluation plan. 


\subsection{Linked Data Quality Model (LDQM)}

The first contribution of the thesis is C1. A Linked Data Quality Model for representing Linked Data quality in a standard manner. H1, the hypothesis related to C1.1, is that the proposed Linked Data Quality Model (LDQM) satisfies a set of characteristics that are relevant for a quality model, namely, comprehensiveness, applicability, understandability, and consistency.

There are no exact criteria to be referred to when evaluating quality models [25]; thus we have selected four different properties based on the various criteria related to quality models described in the literature [25, 196], as well as, based on the criterion that we consider as the most important for the Linked Data domain (i.e., consistency).

\subsubsection{Properties of the Quality Model}

The goal of this evaluation is to verify that the proposed quality model satisfies the properties of a good quality model as discussed before.

Comprehensiveness tends to describe the coverage of the quality model in terms of quality characteristics and quality measures [25], i.e., whether a quality model is complete with respect to the state of the art efforts in quality assessment. To this end, meta publications such as literature surveys of the current quality characteristics and metrics as well as primary studies on specific quality metrics published in the literature can be used to compile a representative list of quality characteristics and metrics for Linked Data. The information gathered this way can be used to verify if the proposed model is representative of the current quality characteristics and metrics.

The quality model presented in this thesis is based on the survey by Zaveri et al. [235] which is, arguably, quite comprehensive with respect to the quality characteristics and quality measures that are evaluated in the state of the art. Since the coverage of the quality model presented in this thesis is in direct relation with the coverage of the survey by Zaveri et al. [235], it can be assumed that the Linked Data quality model is as comprehensive and complete as the mentioned survey. Furthermore, the hierarchy of quality measures in the Linked Data quality model includes measures that are related to various aspects of Linked Data quality (Figure 4.1), covering all the defined aspects. To this extent, the Linked Data quality model proposed in this thesis is also comprehensive in terms of the described Linked Data quality aspects. Furthermore, the Linked Data quality model covers the quality measures in more details than Zaveri et al. [235], and it also describes a higher number of measures. 
Applicability tends to describe the cases in which a quality model has been successfully applied in practice. Since the main purpose of a quality model is to provide guidelines in the evaluation process, as a use case we have developed a tool, LD Sniffer, for evaluating the accessibility of Linked Data (Section 4.3.3). Although the mentioned tool does not cover all the quality measures described in the Linked Data quality model, it has been developed following the quality model guidelines. Furthermore, the evaluation results produced by the tool are in line with the quality model.

Despite the fact that the tool presented in this work is an initial effort in exploiting the Linked Data quality model, it can be assumed that this quality model has potential to be successfully applied in practice as a reference for Linked Data evaluation since parts of it are already used by existing tools and ontologies to represent their evaluation results.

Understandability tends to describe whether a quality model can be easily understood in order to be applied in practice. To this extent, Behkamal et al. argue that an important factor for clear and unambiguous quality models is their hierarchical organization of elements [25], while Bertoa et al. argue that understandability of a quality model is influenced by its structure and organisation [31].

The Linked Data quality model presented in this thesis is a hierarchical quality model, similarly as those defined by the ISO (e.g., ISO 25010 and ISO 25012). Furthermore, in order to address the specific nature of the domain and to reduce the ambiguity that is identified as a problem in generic quality models [8], we have introduced a hierarchy of quality measures and quality characteristics specific to Linked Data, and we have also provided definitions for all the elements as well as, where applicable, formulas for the calculation of quality measures. To this extent, it can be assumed that the Linked Data quality model presented in this thesis is characterised by a high level of understandability.

Consistency tends to describe to which extend the elements of a quality model (e.g., quality characteristics and quality measures) are in agreement and compatibility with each other. Radulovic et al [196] argue that, for a quality model to be consistent, all the formulas in a quality model must contain only those quality measures that are already defined in the model. Furthermore, it is recommendable that all quality measures on lower levels in a hierarchy (i.e., base measures or derived measures) are used for obtaining quality measures on higher levels (i.e., derived measures or indicators), although in some cases measures on lower levels can be stand-alone and bring important information related to particular evaluation. 
The quality model for Linked Data presented in this thesis defines the quality measures and quality characteristics that, according to the guide by Radulovic et al [196]., are completely compatible. All the base measures are used for defining derived measures or indicators, all the derived measures are used for defining indicators, and all the formulas contain only those measures that are defined in the quality model. Furthermore, all the indicators defined in the quality model are used for the measurement of quality characteristics, and all the specified measures are used in formulas. In this sense, it can be concluded that the Linked Data quality model presented in this thesis is consistent.

\subsubsection{Usefulness and Applicability of the Quality Model}

The second hypothesis related to C1. A Linked Data Quality Model is that, H2, LDQM is useful for the development of Linked Data quality assessment tools.

This section presents a practical use case of the usefulness of the Linked Data Quality Model discussed in the thesis. The section presents a command-line tool, LD Sniffer, which allows users to assess the quality of Linked Data resources in a dataset such as DBpedia. Given a set of resource URIs each identified by an IRI, for example, "http://dbpedia.org/resource/Madrid", the tool can dereference the corresponding RDF graph and assess each IRI in the graph using the Linked Data Quality Model. The LD Sniffer tool ${ }^{1}$ is an open-source project under the Apache 2.0 license $^{2}$.

The motivation of presenting this use case is to discuss the usefulness of the model with relation to how a quality model could help the design and implementation of such a tool, how the quality model could improve the understanding of the metrics with explicit semantics and reduce the opportunity for ambiguity and misinterpretations, and finally how the quality model and its formal representation could improve the comparison of the assessment results generated by tools and be used for further tasks such as selection of Linked Data resources with a sufficient quality or recommendation of Linked Data resources.

Use of the quality model The main functionality of the LD Sniffer tool is to generate a quality assessment for a set of given Linked Data resources. Thus, the tool will take a set of URIs of Linked Data resources as the input and generate an output as a set of metrics that provide indications about the quality of the resource. With the aforementioned use case, the first step of the quality assessment tool developer is to identify how the quality of a Linked Data resource can be measured and what metrics can be used to measure quality. The quality model presented in this thesis provides the necessary guidance on that by providing an overview of the different aspects

\footnotetext{
${ }^{1}$ https://github.com/nandana/ld - sniffer

${ }^{2}$ https://WwW.apache.org/licenses/LICENSE- 2.0
} 
that have to be taken into account such as domain data, metadata, vocabularies, interlinking, and infrastructure.

Having an understanding of the different quality aspects allows the assessment tool developers to decide and focus on the aspects that they want to assess. For instance, the initial version of LD Sniffer is planned to be a domain-agnostic general purpose quality assessment tool so it will not focus on quality characteristics such as the accuracy or completeness of facts. It will focus on generic aspects that apply to Linked Data and are described in the previous section, such as Metadata, RDF model, Serialization, and Infrastructure. Later on, the developers of LD Sniffer could decide to add the aspects that they did not consider such as Domain data or Vocabularies used by linking the tool to a domain knowledge base which can verify the accuracy of the facts or the most appropriate ontologies to be reused. Understanding those different aspects allows the Linked Data tool assessment developers to develop their roadmaps using the quality model as guidance In the case of the LD Sniffer tool, the developers are mostly interested in the infrastructure aspect which is highly related to the accessibility dimension.

The second step is to decide which metrics to be used in the quality assessment. The quality model provides a set of metrics that can be used to measure different quality characteristics. In the model, those metrics are described using base measures, derived measures, and indicators. These metrics in the quality model provide insights about which metrics can be implemented by the tool for a given aspect. Furthermore, the base measures provide information to the tool developers on which metrics can be directly evaluated with the subject of the evaluation and the derived measures specify how they can be combined to formulate other measures. Having a Linked Data quality model with a well-defined set of metrics allows the developers to implement those metrics in a standard manner. As the metrics in the quality model are defined as Linked Data with global identifiers and explicit semantics, all tools interpret the metrics in the same way. This allows the LD Sniffer tool to advertise the metrics that it uses in an unambiguous manner. The separation of base measures, derived measures, and indicators also helps the assessment tool developers on how to design the tool. For instance, indicators are important at the time of decision making and, thus, they could be highlighted in the quality assessment results. The Linked Data Quality Model allows finding relevant metrics for a given use case, either via the Linked Data Quality Model wiki ${ }^{3}$ or using the its SPARQL endpoint ${ }^{4}$.

Once the set of metrics are defined and implemented, the next step is the representation of quality results. Having global identifiers for the quality metrics helps in representing the quality results. Dataset validation tools that produce evaluation results that are described using the model proposed in this thesis and reference to

\footnotetext{
${ }^{3}$ http://delicias.dia.fi.upm.es/LDQM/index.php/Accessibility

${ }^{4}$ http://linkeddata.es/resource/ldqm/sparql
} 
the Linked Dada representation of the quality model have the advantage of easier publication of structured results, as well as of reusability of such results.

Figure 8.1 shows an example of some evaluation results that are described according to the ontology proposed in this thesis $(l d q)$, and that reference the RDF representation of the Linked Data quality model $(l d q m)$, while the Appendix provides a coded example and some example SPARQL queries.

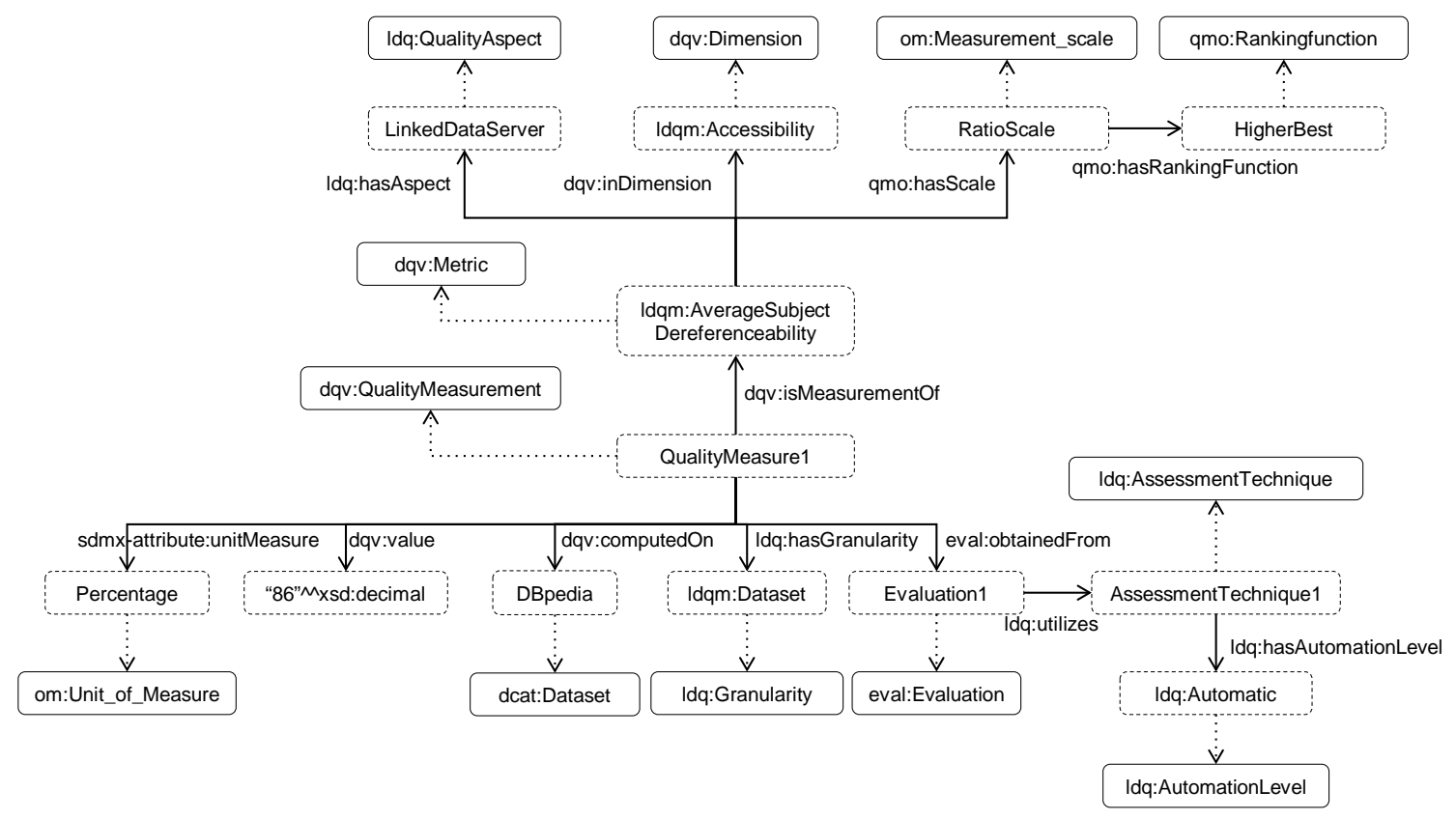

Figure 8.1: Example of dataset evaluation results

Evaluation of DBpedia resources The proposed quality model and the LD sniffer tool were used to perform an assessment of the accessibility of a subset of DBpedia resources. The details of the evaluation are described using LDQM and are available as dereferenceable Linked Data along with a SPARQL endpoint ${ }^{5}$ for querying and as a downloadable RDF dump ${ }^{6}$. The evaluation is focused on the accessibility quality characteristics of the model and uses 8 base measures, 4 derived measures, and 4 indicators as described in the quality model. 1 million IRIs in more than $100 \mathrm{~K}$ resources were assessed to calculate the aforementioned results.

Figure 8.2 shows the distribution of the average subject dereferenceability, average predicate dereferenceability, and average object dereferenceability measurements of the instances in the DBpedia dataset of the dbo:Person and dbo:Place classes, of

\footnotetext{
${ }^{5}$ http://nandana.github.io/ld-sniffer/sparql.html

${ }^{6}$ https : //datahub. io/dataset/ldqm-dbpedia- 2016
} 
the predicates used to describe such instances, and of the objects in those predicates. The $\mathrm{X}$-axis of the line graphs shows the average dereferenceability (rounded to the nearest integer) while the $\mathrm{Y}$ axis shows the number of resources with a given average dereferenceability. In general, both average predicate dereferenceability and average object dereferenceability are commonly in the 90 to 100 range while the average subject dereferenceability varies in a broader range. The main reason for this is the fact that the DBpedia server fails when many requests are sent and it returns a '503 Service Unavailable' error.
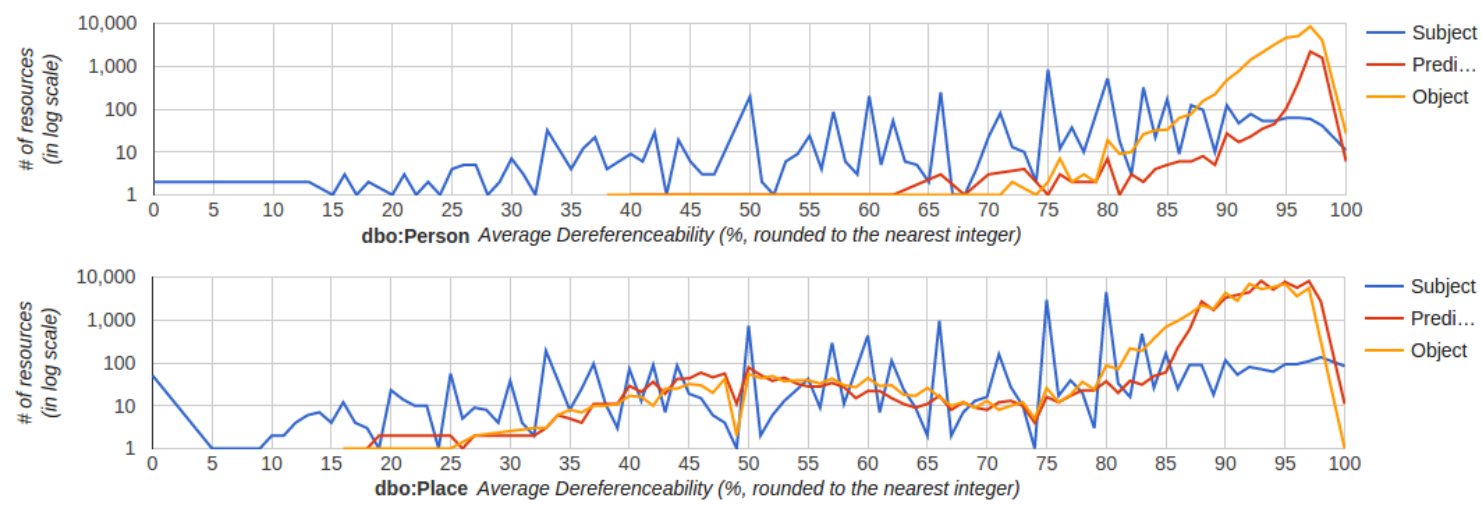

Figure 8.2: Average dereferenceability of dbo:Person/dbo:Place instances

Table 8.1 shows a list of all errors (HTTP status codes) returned by nondereferenceable IRIs in this study. Figure 8.3 shows a pie chart with the most common errors returned when dereferencing IRIs found in the content of dbo:Person and dbo:Place Linked Data resources. The most common status codes include failures in the server to provide a valid response such as '500 Internal Server Error', '502 Bad Gateway', or '503 Service Unavailable' and currently non-exiting IRIs with '404 Not Found', or ' 410 Gone'. More details of the analysis are found in the evaluation results website ${ }^{7}$.

Table 8.2 shows the most common domains non-dereferenceable IRIs in this study and with the number of non-dereferenceable per each domain. As it can be seen, in both dbo:Person and dbo:Place resources the most errors in external IRIs were from $Y A G O$ knowledge base.

\footnotetext{
${ }^{7}$ http://nandana.github.io/ld-sniffer/
} 
Table 8.1: List of errors found in non-dereferenceable IRIs

\begin{tabular}{|l|l|l|l|}
\hline Code & \multicolumn{1}{|c|}{ Description } & Code & \multicolumn{1}{c|}{ Description } \\
\hline 400 & Bad Request & 429 & Too Many Requests \\
\hline 401 & Unauthorized & 451 & Unavailable For Legal Reasons \\
\hline 403 & Forbidden & 456 & Header Field Not Valid \\
\hline 404 & Not Found & 500 & Internal Server Error \\
\hline 405 & Method Not Allowed & 501 & Not Implemented \\
\hline 406 & Not Acceptable & 502 & Bad Gateway \\
\hline 408 & Request Timeout & 503 & Service Unavailable \\
\hline 409 & Conflict & 504 & Gateway Timeout \\
\hline 410 & Gone & 520 & Origin Error \\
\hline 412 & Precondition Failed & 522 & Connection Timed Out \\
\hline 415 & Unsupported Media Type & 523 & Origin is unreachable \\
\hline 416 & Requested Range Not Satisfiable & 524 & A timeout occurred \\
\hline 417 & Expectation Failed & 598 & Network read timeout error \\
\hline
\end{tabular}

Table 8.2: Domains with most non-dereferenceable IRIs

\begin{tabular}{|l|r|l|r|}
\hline \multicolumn{2}{|c|}{ dbo:Person } & \multicolumn{2}{c|}{ dbo:Place } \\
\hline \multicolumn{1}{|c|}{ Domain } & Errors & \multicolumn{1}{c|}{ Domain } & Errors \\
\hline yago-knowledge.org & 34,734 & yago-knowledge.org & 29,494 \\
\hline dbpedia.org & 32,302 & dbpedia.org & 9,402 \\
\hline it.dbpedia.org & 11742 & de.dbpedia.org & 8,486 \\
\hline de.dbpedia.org & 10421 & it.dbpedia.org & 5,923 \\
\hline inkedgeodata.org & 4446 & pt.dbpedia.org & 510 \\
\hline pt.dbpedia.org & 963 & zitgist.com & 230 \\
\hline nl.dbpedia.org & 833 & en.wikipedia.org & 209 \\
\hline gadm.geovocab.org & 785 & es.dbpedia.org & 197 \\
\hline geonames.usgs.gov & 365 & id.dbpedia.org & 149 \\
\hline rdfabout.com & 357 & data.nytimes.com & 142 \\
\hline
\end{tabular}

The main advantage of using, on the one hand, the Linked Data Quality Model for the evaluation and, on the other hand, its RDF representation for representing the information about this evaluation is that it provides reference for the measures to be evaluated and a large amount of provenance information for understanding how the evaluation was performed. For instance, when a metric such as "average subject dereferenceability" 8 is dereferenced it provides information such as the definition of

\footnotetext{
${ }^{8}$ http ://linkeddata.es/page/resource/ldqm/QualityIndicator/
} 

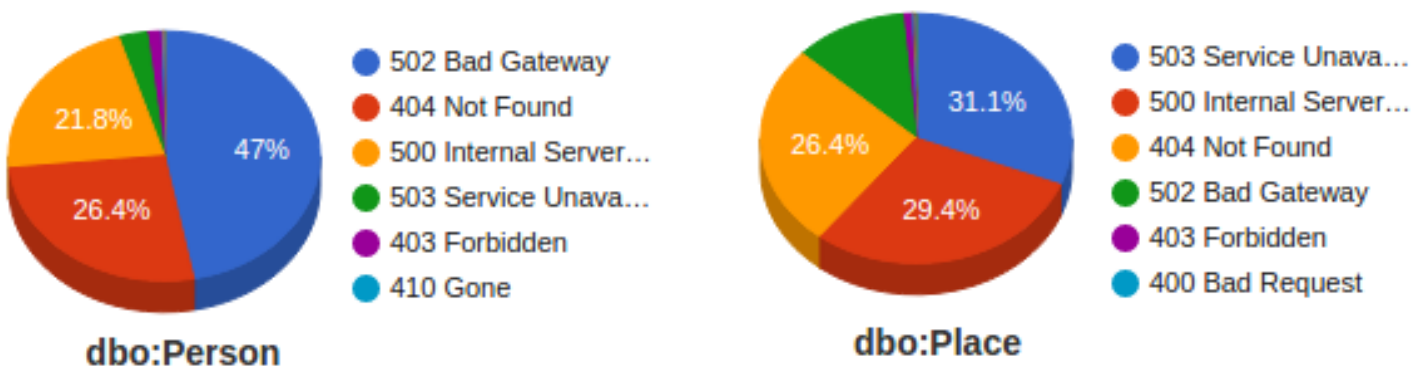

Figure 8.3: Distribution of HTTP status codes for non-dereferenceable IRIs

the metric, its description, the base measures or derived measures used for calculating the given indicator, the scale, the relevant quality characteristic, and the Linked Data aspect. In addition, the base measures such as "number of distinct subjects" 9 provide the technique that was used to calculate the base measure. This additional information helps the quality evaluation result consumers to get a better understanding of the evaluation results and utilise them for making decisions.

\subsection{A Framework for Linked Data profiling (Loupe)}

The second contribution of the thesis is C2. The Loupe Linked Data profiling framework. In this section, we validate the hypotheses $\mathrm{H}_{3}$ and $\mathrm{H}_{4}$ related to $\mathrm{C}_{2}$ according to the evaluation plan discussed in Section 3.5.

\subsubsection{Expressivity of the Loupe Extended Dataset Descrip- tion Model}

The first hypothesis related to the contribution C2, as presented in Chapter 3, is that $\boldsymbol{H} 3$ the proposed Loupe dataset description model (C2.1) is more expressive than the current dataset description models and contains fine-grained Linked Data profiling metrics needed for quality assessment techniques and repair use cases

To this end, we evaluate the expressiveness of the Loupe extended dataset description model for representing Linked Data profiling metrics in RDF based on the profiling tasks and derived metrics that were identified in Section 5.2. There are six

Averagesubjectdereferenceability

${ }^{9}$ http://linkeddata.es/page/resource/ldqm/BaseMeasure/Numberofdistinctsubjects' 
main Linked Data profiling tasks were identified: (T1.) RDF Triple Analysis, (T2.) Vocabulary Usage Analysis, (T3.) Network Metrics Analysis, (T4.) Triple Pattern Analysis (T5.) Value Distribution Analysis, and (T6.) Language Analysis.

The state of the art dataset description models included in the evaluation are listed in Table 8.3. DCAT [152], the data catalogue vocabulary published by W3C for facilitating interoperability between data catalogs published on the Web, is not included in this comparison because it does not contain information specific to RDF. The DCAT vocabulary is designed to be suitable for data of any format such as XML, CSV, JSON, or other formats. Because this evaluation is focused on RDF specific profiling tasks and metrics, DCAT is not part of the evaluation.

It is also important to note that the objective of this evaluation is to validate the expressivity of the aforementioned vocabularies only from the perspective of the data profiling tasks and metrics identified in this thesis. Each of the models evaluated in this section covers a wide variety of other metadata that is not in the scope of this evaluation such as licence metadata, accessibility metadata, information about the distributions, provenance information, etc.

Table 8.3: State-of-the-art dataset description models

\begin{tabular}{|l|l|}
\hline Model & Description \\
\hline RDFStats [143] & $\begin{array}{l}\text { RDFStats is a framework for generating statistics from RDF } \\
\text { data that can be used for query processing and optimisation over } \\
\text { SPARQL endpoints. The generated statistics are described using } \\
\text { the RDFStats vocabulary. }\end{array}$ \\
\hline VoID [132] & $\begin{array}{l}\text { VoID is an RDF Schema vocabulary for expressing metadata } \\
\text { about RDF datasets and it is currently one of the most widely } \\
\text { used dataset description model with RDF data. }\end{array}$ \\
\hline HoID-Ext [153] & $\begin{array}{l}\text { The VoID-ext vocabulary is a VoID extension to allow much } \\
\text { richer statistical data about RDF datasets and it is developed to } \\
\text { be used by the Aether tool. }\end{array}$ \\
\hline $\begin{array}{l}\text { LDSO (LOD- } \\
\text { Stats) [70] }\end{array}$ & $\begin{array}{l}\text { Dataset Descriptions: HCLS Community Profile is a dataset } \\
\text { description model developed by the W3C Semantic Web in Health } \\
\text { Care and Life Sciences Interest Group. Rather than defining a } \\
\text { new vocabulary the HCLS community profile reuses terms from } \\
\text { existing vocabularies such as the Dublin Core Metadata Initiative } \\
\text { (DCMI), DCAT, PROV-O, VoID, VoID-ext, and Schema.org. }\end{array}$ \\
\hline $\begin{array}{l}\text { The LODStats Dataset Vocabulary (LDSO) vocabulary is de- } \\
\text { veloped to represent the RDF dataset statistics information ex- } \\
\text { tracted by the LODStats tool. }\end{array}$ \\
\hline
\end{tabular}

Table 8.3 - Continued on next page 
Table 8.3 - Continued from previous page

\begin{tabular}{|l|l|}
\hline Model & Description \\
\hline $\begin{array}{l}\text { LLM (LOD } \\
\text { Laundro- } \\
\text { mat) }[145]\end{array}$ & $\begin{array}{l}\text { The LOD Laundromat meta-dataset (LLM) vocabulary is a vo- } \\
\text { cabulary that is developed to describe the metadata about the } \\
\text { datasets stored in LOD Laundromat. }\end{array}$ \\
\hline DataID [44] & $\begin{array}{l}\text { DataID is a metadata description model to enabling users to } \\
\text { discover, understand and process the available data and it is } \\
\text { based on the DCAT and VoID vocabularies. }\end{array}$ \\
\hline ABSTAT [214] & $\begin{array}{l}\text { ABSTAT is an ontology-driven data abstraction tool that sup- } \\
\text { ports the extraction and the representation of summaries of linked } \\
\text { datasets using data profiling. }\end{array}$ \\
\hline
\end{tabular}

Table 8.4 shows the coverage of Linked Data profiling metrics in each of the dataset description models we discussed before. As it can be seen from the table, even though metrics related to RDF Triple Analysis and Vocabulary Usage Analysis tasks are partially covered by most dataset description models, other metrics such as Network Metrics, Triple Patterns, Value Distribution and Language analysis is largely not covered by other models. Noticeably, the LLM model partially covers this aspect. Nevertheless, as Table 8.4, there is a gap in coverage related to Linked Data profiling metrics in the current state-of-the-art dataset description models.

Table 8.4: Summary of metrics coverage

\begin{tabular}{|c|c|c|c|c|c|c|c|c|c|}
\hline \multirow{2}{*}{ Task } & \multirow{2}{*}{$\begin{array}{l}\text { \# of } \\
\text { metric: }\end{array}$} & \multicolumn{8}{|c|}{ Coverage } \\
\hline & & $\begin{array}{l}\text { RDF } \\
\text { Stats }\end{array}$ & VoID & $\begin{array}{l}\text { VoID- } \\
\text { Ext }\end{array}$ & $\begin{array}{l}\text { HCLS } \\
\text { Profile }\end{array}$ & LDSO & LLM & DataID & Loupe \\
\hline $\begin{array}{l}\text { RDF Triple Analy- } \\
\text { sis (T1) }\end{array}$ & 20 & 2 & 4 & 14 & 4 & 5 & 18 & 4 & 20 \\
\hline $\begin{array}{l}\text { Vocabulary Usage } \\
\text { Analysis (T2) }\end{array}$ & 18 & 6 & 8 & 8 & 7 & 4 & 7 & 2 & 18 \\
\hline $\begin{array}{l}\text { Network Metric } \\
\text { Analysis (T3) }\end{array}$ & 13 & - & - & - & - & - & 10 & - & 13 \\
\hline $\begin{array}{l}\text { Triple Pattern Anal- } \\
\text { ysis (T4) }\end{array}$ & 9 & - & - & - & - & - & - & - & 9 \\
\hline $\begin{array}{l}\text { Value Distribution } \\
\text { Analysis (T5) }\end{array}$ & 20 & - & - & 6 & - & - & 12 & - & 20 \\
\hline $\begin{array}{l}\text { Language Analysis } \\
\text { (T6) }\end{array}$ & 4 & - & - & 3 & - & 2 & 2 & - & 4 \\
\hline
\end{tabular}


Rest of this section shows detailed tables for coverage of metrics in each task, more concretely, Table 8.5 shows coverage for RDF Triple Analysis, Table 8.6 for Vocabulary Usage Analysis, Table 8.7 for Network Metrics Analysis, Table 8.8 for Triple Pattern Analysis, Table 8.9 for Value Distribution Analysis, and Table 8.10 for Language Analysis.

Table 8.5: T1 - RDF triple analysis

\begin{tabular}{|c|c|c|c|c|c|c|c|c|c|}
\hline ID & Name & $\begin{array}{l}\text { RDF } \\
\text { Stats }\end{array}$ & VoID & $\begin{array}{l}\text { VoID } \\
\text {-Ext }\end{array}$ & $\begin{array}{l}\text { HCLS } \\
\text { Profile }\end{array}$ & LDSO & LLM & DataII & Loupe \\
\hline T.1.1. & $\begin{array}{l}\text { Dataset Triple } \\
\text { Count }\end{array}$ & $\checkmark$ & $\checkmark$ & $\checkmark$ & $\checkmark$ & $\checkmark$ & $\checkmark$ & $\checkmark$ & $\checkmark$ \\
\hline T1.1.2 & $\begin{array}{l}\text { Duplicate Triple } \\
\text { Count }\end{array}$ & & & & & & $\checkmark$ & & $\checkmark$ \\
\hline T1.2.1 & $\begin{array}{ll}\text { Named } & \text { Graph } \\
\text { URIs } & \end{array}$ & & & & & $\checkmark$ & & & $\begin{array}{lll} \\
\checkmark\end{array}$ \\
\hline T1.2.2 & $\begin{array}{l}\text { Named Graph } \\
\text { Triple Count }\end{array}$ & & & & & $\checkmark$ & & & $\checkmark$ \\
\hline T1.3.1 & $\begin{array}{l}\text { Distinct Subject } \\
\text { Count }\end{array}$ & $\checkmark$ & $\checkmark$ & $\checkmark$ & $\checkmark$ & & $\checkmark$ & $\checkmark$ & $\checkmark$ \\
\hline T1.3.2 & $\begin{array}{ll}\text { IRI } & \text { Subject } \\
\text { Count } & \end{array}$ & & $\checkmark$ & $\checkmark$ & & & $\checkmark$ & & $\checkmark$ \\
\hline T1.3.3 & $\begin{array}{l}\text { Distinct IRI Sub- } \\
\text { ject Count }\end{array}$ & & & $\checkmark$ & & & $\checkmark$ & & $\checkmark$ \\
\hline T1.3.4 & $\begin{array}{l}\text { Blanknode Sub- } \\
\text { ject Count }\end{array}$ & & & $\checkmark$ & & & $\checkmark$ & & $\checkmark$ \\
\hline T1.3.5 & $\begin{array}{lr}\text { Distinct } & \text { Blan- } \\
\text { knode } & \text { Subject } \\
\text { Count } & \\
\end{array}$ & & & $\checkmark$ & & & $\checkmark$ & & $\checkmark$ \\
\hline T1.4.1 & $\begin{array}{l}\text { Distinct Object } \\
\text { Count }\end{array}$ & & $\checkmark$ & $\checkmark$ & $\checkmark$ & & $\checkmark$ & $\checkmark$ & $\checkmark$ \\
\hline T1.4.2 & $\begin{array}{ll}\text { IRI } & \text { Object } \\
\text { Count } & \end{array}$ & & & $\checkmark$ & & & $\checkmark$ & & $\checkmark$ \\
\hline T1.4.3 & $\begin{array}{l}\text { Distinct IRI Ob- } \\
\text { ject Count }\end{array}$ & & & $\checkmark$ & & & $\checkmark$ & & $\checkmark$ \\
\hline T1.4.4 & $\begin{array}{l}\text { Blanknode Ob- } \\
\text { ject Count }\end{array}$ & & & $\checkmark$ & & & $\checkmark$ & & $\checkmark$ \\
\hline
\end{tabular}

Table 8.5 - Continued on next page 
Table 8.5 - Continued from previous page

\begin{tabular}{|l|l|l|l|l|l|l|l|l|l|}
\hline ID & Name & $\begin{array}{l}\text { RDF } \\
\text { Stats }\end{array}$ & VoID & $\begin{array}{l}\text { VoID } \\
\text {-Ext }\end{array}$ & $\begin{array}{l}\text { HCLS } \\
\text { Profile }\end{array}$ & LDSO & LLM & DataID Loupe \\
\hline T1.4.5 & $\begin{array}{l}\text { Distinct Blan- } \\
\text { knode Object } \\
\text { Count }\end{array}$ & & & $\checkmark$ & & & $\checkmark$ & & $\checkmark$ \\
\hline T1.4.6 & $\begin{array}{l}\text { Literal Object } \\
\text { Count }\end{array}$ & & & $\checkmark$ & & $\checkmark$ & $\checkmark$ & & $\checkmark$ \\
\hline T1.4.7 & $\begin{array}{l}\text { Distinct Literal } \\
\text { Object Count }\end{array}$ & & $\checkmark$ & & & $\checkmark$ & & $\checkmark$ \\
\hline T1.5 & Total RDF Node & & $\checkmark$ & & & $\checkmark$ & & $\checkmark$ \\
\hline T1.6 & $\begin{array}{l}\text { totalBlankNodes } \\
\text { T1.7 }\end{array}$ & $\begin{array}{l}\text { Typed Entity } \\
\text { Count }\end{array}$ & & & & $\checkmark$ & $\checkmark$ & & $\checkmark$ \\
\hline T1.8 & $\begin{array}{l}\text { Labeled Entity } \\
\text { Count }\end{array}$ & & & & $\checkmark$ & & & $\checkmark$ \\
\hline
\end{tabular}

Table 8.6: T2. Vocabulary usage analysis

\begin{tabular}{|c|c|c|c|c|c|c|c|c|c|}
\hline ID & Name & $\begin{array}{l}\text { RDF } \\
\text { Stats }\end{array}$ & VoID & $\begin{array}{l}\text { VoID } \\
\text {-Ext }\end{array}$ & $\begin{array}{l}\text { HCLS } \\
\text { Profile }\end{array}$ & LDSO & LLM & DataID & Loupe \\
\hline $\mathrm{T} 2.1$ & $\begin{array}{l}\text { Vocabulary } \\
\text { Count }\end{array}$ & & & & & & & & $\checkmark$ \\
\hline $\mathrm{T} 2.2$ & Class Count & & $\checkmark$ & $\checkmark$ & & & $\checkmark$ & $\checkmark$ & $\checkmark$ \\
\hline $\mathrm{T} 2.3$ & Class List & $\checkmark$ & $\checkmark$ & $\checkmark$ & $\checkmark$ & $\checkmark$ & $\checkmark$ & & $\checkmark$ \\
\hline T2.4 & Property Count & & $\checkmark$ & & & & $\checkmark$ & & $\checkmark$ \\
\hline $\mathrm{T} 2.5$ & Property List & $\checkmark$ & $\checkmark$ & $\checkmark$ & $\checkmark$ & $\checkmark$ & $\checkmark$ & & $\checkmark$ \\
\hline T2.6 & $\begin{array}{l}\text { Object Property } \\
\text { Count }\end{array}$ & & & & & & & & $\checkmark$ \\
\hline $\mathrm{T} 2.7$ & $\begin{array}{l}\text { Datatype Prop- } \\
\text { erty Count }\end{array}$ & & & & & & & & $\checkmark$ \\
\hline T2.8 & $\begin{array}{l}\text { Defined Class } \\
\text { Count }\end{array}$ & & & & & & $\checkmark$ & & $\checkmark$ \\
\hline $\mathrm{T} 2.9$ & $\begin{array}{l}\text { Defined Property } \\
\text { Count }\end{array}$ & & & & & & $\checkmark$ & & $\checkmark$ \\
\hline $\mathrm{T} 2.10 .1$ & $\begin{array}{l}\text { Vocabulary } \\
\text { Namespace }\end{array}$ & & $\checkmark$ & $\checkmark$ & $\checkmark$ & $\checkmark$ & $\checkmark$ & $\checkmark$ & $\checkmark$ \\
\hline
\end{tabular}

Table 8.6 - Continued on next page 
Table 8.6 - Continued from previous page

\begin{tabular}{|l|l|l|l|l|l|l|l|l|l|}
\hline ID & Name & $\begin{array}{l}\text { RDF } \\
\text { Stats }\end{array}$ & VoID & $\begin{array}{l}\text { VoID } \\
\text {-Ext }\end{array}$ & $\begin{array}{l}\text { HCLS } \\
\text { Profile }\end{array}$ & LDSO & LLM & DataID Loupe \\
\hline T2.10.2 & $\begin{array}{l}\text { Vocabulary } \\
\text { Class Count }\end{array}$ & & & & & & & & $\checkmark$ \\
\hline T2.10.3 & $\begin{array}{l}\text { Vocabulary } \\
\text { Property Count }\end{array}$ & & & & & & & & $\checkmark$ \\
\hline T2.11.1 & Class IRI & $\checkmark$ & & $\checkmark$ & $\checkmark$ & $\checkmark$ & & & $\checkmark$ \\
\hline T2.11.2 & Instance Count & $\checkmark$ & $\checkmark$ & $\checkmark$ & $\checkmark$ & & & & $\checkmark$ \\
\hline T2.11.3 & Instance Ratio & & & & & & & & $\checkmark$ \\
\hline T2.12.1 & PropertyIRI & $\checkmark$ & $\checkmark$ & $\checkmark$ & $\checkmark$ & & & & $\checkmark$ \\
\hline T2.12.2 & Triple Count & $\checkmark$ & $\checkmark$ & $\checkmark$ & $\checkmark$ & & & & $\checkmark$ \\
\hline T2.12.3 & Triple Ratio & & & & & & & & $\checkmark$ \\
\hline
\end{tabular}

Table 8.7: T3. Network metrics analysis

\begin{tabular}{|l|l|l|l|l|l|l|l|l|l|}
\hline ID & Name & $\begin{array}{c}\text { RDF } \\
\text { Stats }\end{array}$ & VoID & $\begin{array}{l}\text { VoID } \\
\text {-Ext }\end{array}$ & $\begin{array}{c}\text { HCLS } \\
\text { Profile }\end{array}$ & LDSO & LLM & DataID Loupe \\
\hline T3.1.1 & Mean indegree & & & & & & $\checkmark$ & & $\checkmark$ \\
\hline T3.1.2 & Max indegree & & & & & & $\checkmark$ & & $\checkmark$ \\
\hline T3.1.3 & Min indegree & & & & & & $\checkmark$ & & $\checkmark$ \\
\hline T3.1.4 & Mode indegree & & & & & & $\checkmark$ & & $\checkmark$ \\
\hline T3.1.5 & $\begin{array}{l}\text { Std. deviation of } \\
\text { indegree }\end{array}$ & & & & & $\checkmark$ & & $\checkmark$ \\
\hline T3.2.1 & Mean outdegree & & & & & $\checkmark$ & & $\checkmark$ \\
\hline T3.2.2 & Max outdegree & & & & & & $\checkmark$ & & $\checkmark$ \\
\hline T3.2.3 & Min outdegree & & & & & & $\checkmark$ & & $\checkmark$ \\
\hline T3.2.4 & Mode outdegree & & & & & & $\checkmark$ & & $\checkmark$ \\
\hline T3.2.5 & $\begin{array}{l}\text { Std. deviation of } \\
\text { outdegree }\end{array}$ & & & & & $\checkmark$ & & $\checkmark$ \\
\hline T3.3 & $\begin{array}{l}\text { Component } \\
\text { Count }\end{array}$ & & & & & & & & $\checkmark$ \\
\hline T3.4 & $\begin{array}{l}\text { Largest Compo- } \\
\text { nent Size }\end{array}$ & & & & & & & $\checkmark$ \\
\hline T3.5 & $\begin{array}{l}\text { Largest Compo- } \\
\text { nent Ratio }\end{array}$ & & & & & & & $\checkmark$ \\
\hline
\end{tabular}


Table 8.8: T4. Triple pattern analysis

\begin{tabular}{|c|c|c|c|c|c|c|c|c|c|}
\hline ID & Name & $\begin{array}{l}\text { RDF } \\
\text { Stats }\end{array}$ & VoID & $\begin{array}{l}\text { VoID } \\
\text {-Ext }\end{array}$ & $\begin{array}{l}\text { HCLS } \\
\text { Profile }\end{array}$ & LDSO & LLM & DataIl & Loupe \\
\hline T4.1.1 & $\begin{array}{l}\text { Class Property } \\
\text { Triple Count }\end{array}$ & & & & & & & & $\checkmark$ \\
\hline T4.1.2 & $\begin{array}{l}\text { Class-property } \\
\text { Triple Ratio }\end{array}$ & & & & & & & & $\checkmark$ \\
\hline T4.2.1 & $\begin{array}{l}\text { Property-class } \\
\text { Triple Count }\end{array}$ & & & & & & & & $\checkmark$ \\
\hline T4.2.2 & $\begin{array}{l}\text { Property-class } \\
\text { Triple Ratio }\end{array}$ & & & & & & & & $\checkmark$ \\
\hline T4.3.1 & Subject Type & & & & & & & & $\checkmark$ \\
\hline T4.3.2 & Predicate & & & & & & & & $\checkmark$ \\
\hline T4.3.3 & Object Type & & & & & & & & $\checkmark$ \\
\hline T4.3.4 & $\begin{array}{l}\text { Abstract Triple } \\
\text { Pattern Count }\end{array}$ & & & & & & & & $\checkmark$ \\
\hline T4.3.5 & $\begin{array}{l}\text { Abstract Triple } \\
\text { Pattern Ratio }\end{array}$ & & & & & & & & $\checkmark$ \\
\hline
\end{tabular}

Table 8.9: T5. Value distribution analysis

\begin{tabular}{|l|l|l|l|l|l|l|l|l|l|}
\hline ID & Name & $\begin{array}{l}\text { RDF } \\
\text { Stats }\end{array}$ & VoID & $\begin{array}{l}\text { VoID } \\
\text {-Ext }\end{array}$ & $\begin{array}{l}\text { HCLS } \\
\text { Profile }\end{array}$ & LDSO & LLM & DataID Loupe \\
\hline T5.1.1 & Min value & & & & & & $\checkmark$ & & $\checkmark$ \\
\hline T5.1.2 & Max value & & & & & & $\checkmark$ & & $\checkmark$ \\
\hline T5.1.3 & Mean value & & & & & & $\checkmark$ & & $\checkmark$ \\
\hline T5.1.4 & Median value & & & & & & & & $\checkmark$ \\
\hline T5.1.5 & Std. deviation \\
\hline T5.2.1 string & $\begin{array}{l}\text { Min string } \\
\text { length }\end{array}$ & & $\checkmark$ & & & $\checkmark$ & & $\checkmark$ \\
\hline T5.2.2 & $\begin{array}{l}\text { Max } \\
\text { length string }\end{array}$ & & $\checkmark$ & & & $\checkmark$ & & $\checkmark$ \\
\hline T5.2.3 & $\begin{array}{l}\text { Mean } \\
\text { length }\end{array}$ & & $\checkmark$ & & & $\checkmark$ & & $\checkmark$ \\
\hline T5.2.4 & $\begin{array}{l}\text { Median string } \\
\text { length }\end{array}$ & & & & & & & $\checkmark$ \\
\hline
\end{tabular}

Table 8.9 - Continued on next page 
Table 8.9 - Continued from previous page

\begin{tabular}{|l|l|l|l|l|l|l|l|l|l|}
\hline ID & Name & $\begin{array}{l}\text { RDF } \\
\text { Stats }\end{array}$ & VoID & $\begin{array}{l}\text { VoID } \\
\text {-Ext }\end{array}$ & $\begin{array}{l}\text { HCLS } \\
\text { Profile }\end{array}$ & LDSO & LLM & DataID Loupe \\
\hline T5.2.5 & $\begin{array}{l}\text { Mode string } \\
\text { length }\end{array}$ & & & & & & & $\checkmark$ \\
\hline T5.2.6 & $\begin{array}{l}\text { String length } \\
\text { Std. deviation }\end{array}$ & & & & & $\checkmark$ & & $\checkmark$ \\
\hline T5.3.1 & Min IRI length & & & $\checkmark$ & & & $\checkmark$ & & $\checkmark$ \\
\hline T5.3.2 & Max IRI length & & $\checkmark$ & & & $\checkmark$ & & $\checkmark$ \\
\hline T5.3.3 & Mean IRI length & & $\checkmark$ & & & $\checkmark$ & & $\checkmark$ \\
\hline T5.3.4 & $\begin{array}{l}\text { Median IRI } \\
\text { length }\end{array}$ & & & & & & & $\checkmark$ \\
\hline T5.3.5 & Mode IRI length & & & & & & & $\checkmark$ \\
\hline T5.3.6 length Std. & $\begin{array}{l}\text { lRI } \\
\text { deviation }\end{array}$ & & & & & $\checkmark$ & & $\checkmark$ \\
\hline T5.4.1 & $\begin{array}{l}\text { Common names- } \\
\text { pace IRI }\end{array}$ & & & & & & & $\checkmark$ \\
\hline T5.4.2 & $\begin{array}{l}\text { Common names- } \\
\text { pace Count }\end{array}$ & & & & & & & $\checkmark$ \\
\hline T5.4.3 & $\begin{array}{l}\text { Common names- } \\
\text { pace Ratio }\end{array}$ & & & & & & & $\checkmark$ \\
\hline
\end{tabular}

Table 8.10: T6. Language analysis

\begin{tabular}{|l|l|l|l|l|l|l|l|l|l|}
\hline ID & Name & $\begin{array}{l}\text { RDF } \\
\text { Stats }\end{array}$ & VoID & $\begin{array}{l}\text { VoID } \\
\text {-Ext }\end{array}$ & $\begin{array}{l}\text { HCLS } \\
\text { Profile }\end{array}$ & LDSO & LLM & DataID Loupe \\
\hline T6.1.1 & Language & & & $\checkmark$ & & $\checkmark$ & $\checkmark$ & & $\checkmark$ \\
\hline T6.1.2 & String count & & & $\checkmark$ & & $\checkmark$ & $\checkmark$ & & $\checkmark$ \\
\hline T6.1.3 & $\begin{array}{l}\text { Distinct string } \\
\text { count }\end{array}$ & & & $\checkmark$ & & & & & $\checkmark$ \\
\hline T6.1.4 & String ratio & & & & & & & & $\checkmark$ \\
\hline
\end{tabular}

\subsubsection{Scalability of the Loupe Tools}

The second hypothesis related to the contribution C2, as presented in Chapter 3, is that $\boldsymbol{H}_{\mathbf{4}}$, the Loupe Linked Data profiling tools (C2.2) are scalable, i.e., the execution 
time of the Loupe Linked Data tools scale with respect to the dataset size (in triples) and richness of vocabularies used (number of distinct classes and properties) and it can be used to profile large datasets (more than million triples) in the Linked Open Data cloud in less than 30 minutes.

Experimental setup: The methodology used for this evaluation is to select a representative sample of the publicly available datasets as the input and preform the profiling activities to record the execution times per each of the datasets. As the sample of representative datasets, we have collected all the datasets available in the LOD Laundromat repository that is widely considered by the community as a representative sample of the datasets available [201]. In total, 34 billions of triples from 26 thousand datasets were used for the evaluation. A summary of the dataset is presented in Table 8.11.

The experiments are performed in a computer with 8 cores and the frequency range is $2.53 \mathrm{GHz}$ to $1.59 \mathrm{GHz}$. The server was equipped with a $16 \mathrm{~GB}$ of physical memory. The operating system was Ubuntu 16.04.4 LTS and Java 1.8.0_162 version. The open source edition of the Virtuoso server was used for SPARQL queries.

Table 8.11: Summary of input datasets

\begin{tabular}{|l|r|}
\hline Description & Value \\
\hline Number of datasets & 26,063 \\
\hline Number of triples & $34,556,443,529$ \\
\hline Min dataset size (triples) & 1 \\
\hline Max dataset size (triples) & $571,825,316)$ \\
\hline Median dataset size & 109,269 \\
\hline Average dataset size & $1,325,881$ \\
\hline
\end{tabular}

In the first part of the evaluation, the execution time is compared against the dataset size measured in the number of triples. The motivation for using the size of the dataset is because most metrics in the profiling tasks are influenced by the size of the dataset. The size of the datasets used for the evaluation varies from datasets with a single triple to datasets with 571 million triples. Because there are a large number of datasets with the same number of triples (especially for the datasets with a smaller number of triples such as 100 triples), the average execution of all datasets with the same number of triples are calculated. Figure 8.4 illustrates the execution time (in seconds) of the data profiling tool against the dataset size (in millions of triples). The execution time varies from a few milliseconds to several minutes. The largest dataset took 13 minutes to finish. As it can be seen from Figure 8.4, the execution time gradually increases with the dataset size but does not increase exponentially (it shows rather a linear increase). For smaller datasets, there is a noticeable variation 
of the execution time. For smaller datasets, the execution time can be affected by many other factors such as the number of different vocabularies used, the ability to run the profiling tasks in parallel, etc. Nevertheless, for large datasets the effect of these factors becomes less and the execution time shows a more direct relation with the number of triples.

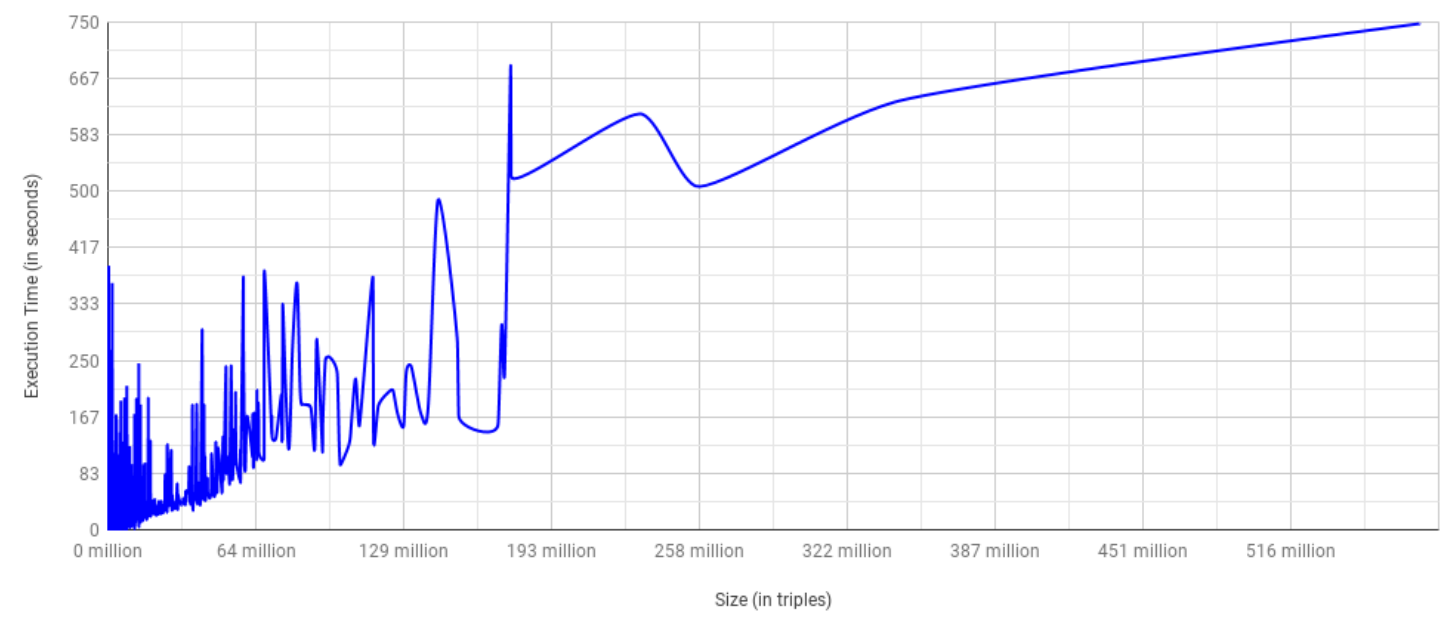

Figure 8.4: Execution time (in seconds) Vs dataset size (in millions of triples)

Similarly, Figure 8.5 illustrates the average execution time per triple vs the size of the dataset in triples. Because of the large variation in the scale, the horizontal axis of the graph is plotted using the log scale. It shows that the average time varies for smaller datasets by it stabilises for larger datasets. Using the same information, we have calculated the correlation between the dataset size and execution time as illustrated in Figure 8.6. There is a positive correlation with a correlation coefficient of 0.74 ( $\mathrm{P}<0.0001)$. Even though correlation does not necessarily imply causation, this shows strong evidence that the execution time increases somewhat linear manner with the dataset size.

In the second evaluation, we look at how the execution time changes with the number of vocabulary terms used in the dataset. By vocabulary terms, we refer to the classes and properties used (i.e., TBox or the schema) in the dataset. The motivation for exploring how the profiling tasks scale with respect to the vocabulary terms is the fact that many profiling tasks are directly dependent on the vocabulary terms. Thus, depending on the vocabulary terms, the number of atomic profiling tasks performed (e.g., the number of queries to extract data) varies. In this evaluation, we have summed up the number of classes and the number of properties used in the dataset to generate the total number of vocabulary terms used. Similar to the previous case of dataset size in triples, when two or more datasets have the same number of 


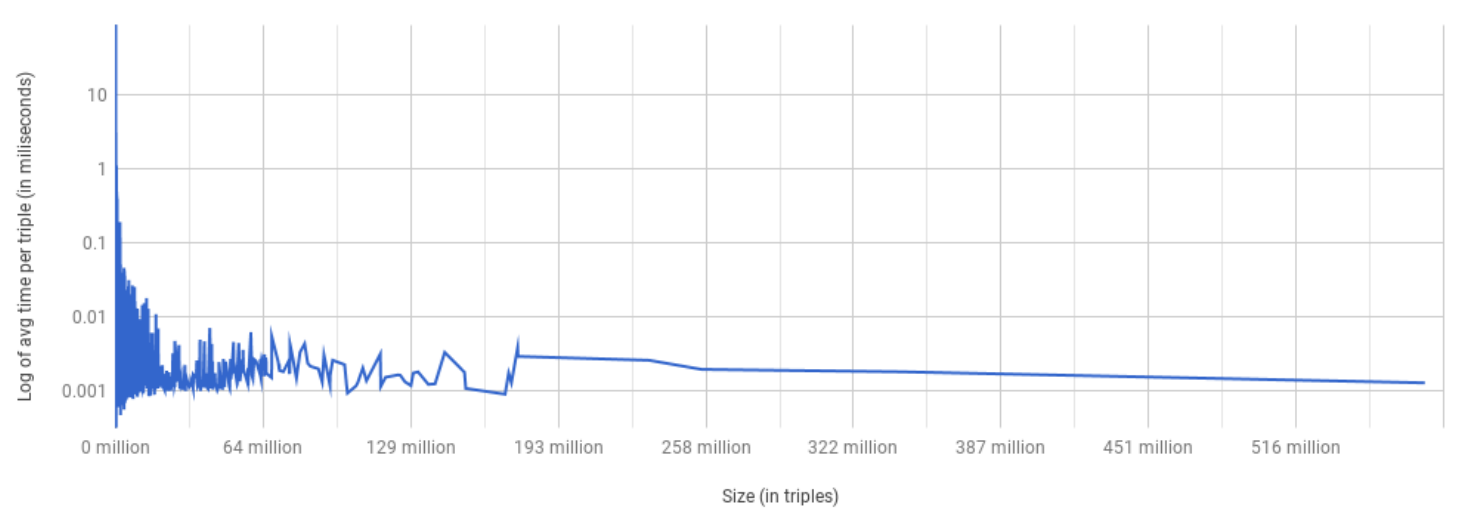

Figure 8.5: Log of avg time per triple (in milliseconds) Vs dataset size (in millions of triples)

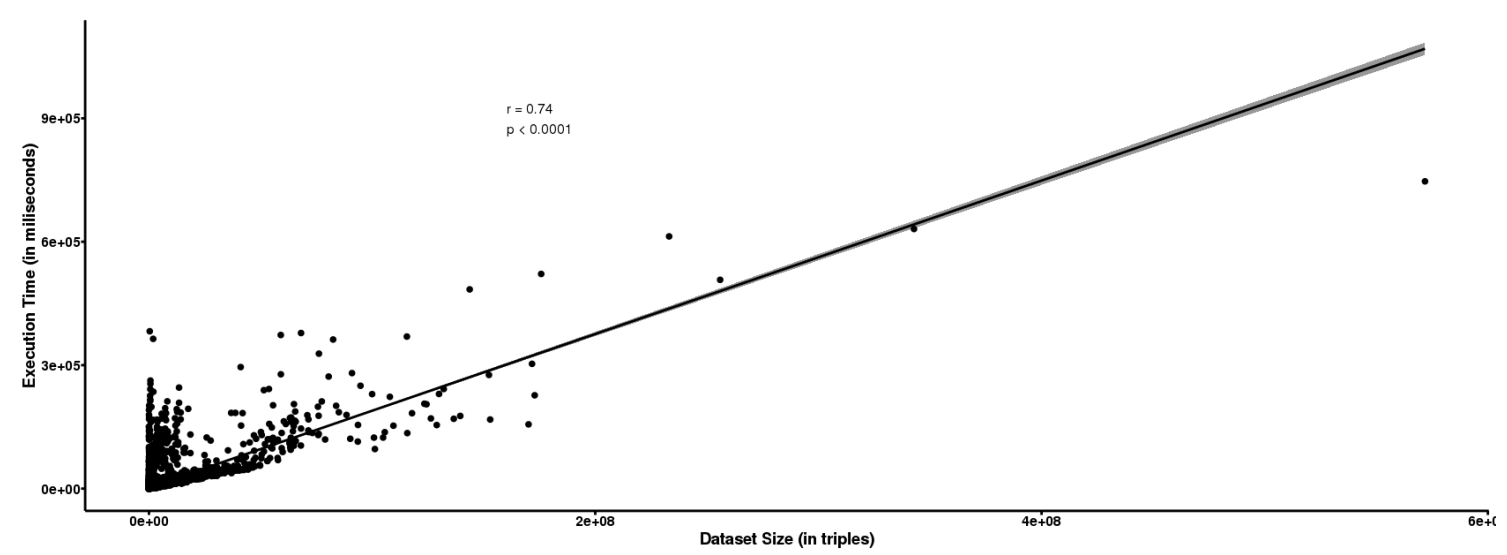

Figure 8.6: Correlation between dataset size and execution time

vocabulary terms, the average execution time of all such datasets were considered. The summary of the distribution of vocabulary term count in each dataset is shown in Table 8.12 .

Table 8.12: Summary of vocabulary term counts

\begin{tabular}{|l|r|}
\hline Description & Value \\
\hline Number of datasets & 26,063 \\
\hline Distinct vocabulary term count & 1,056 \\
\hline Minimum vocabulary term count & 1 \\
\hline Maximum vocabulary term count & 369,145 \\
\hline Median dataset size & 1484 \\
\hline
\end{tabular}




\begin{tabular}{|l|r|}
\hline Average dataset size & 4505 \\
\hline
\end{tabular}

How the execution time changes with the increase of the total number of vocabulary terms is shown in Figure 8.7. Similar to the dataset size, we see a gradual increase in execution time when the number of vocabulary terms are increased but the growth is not exponential.

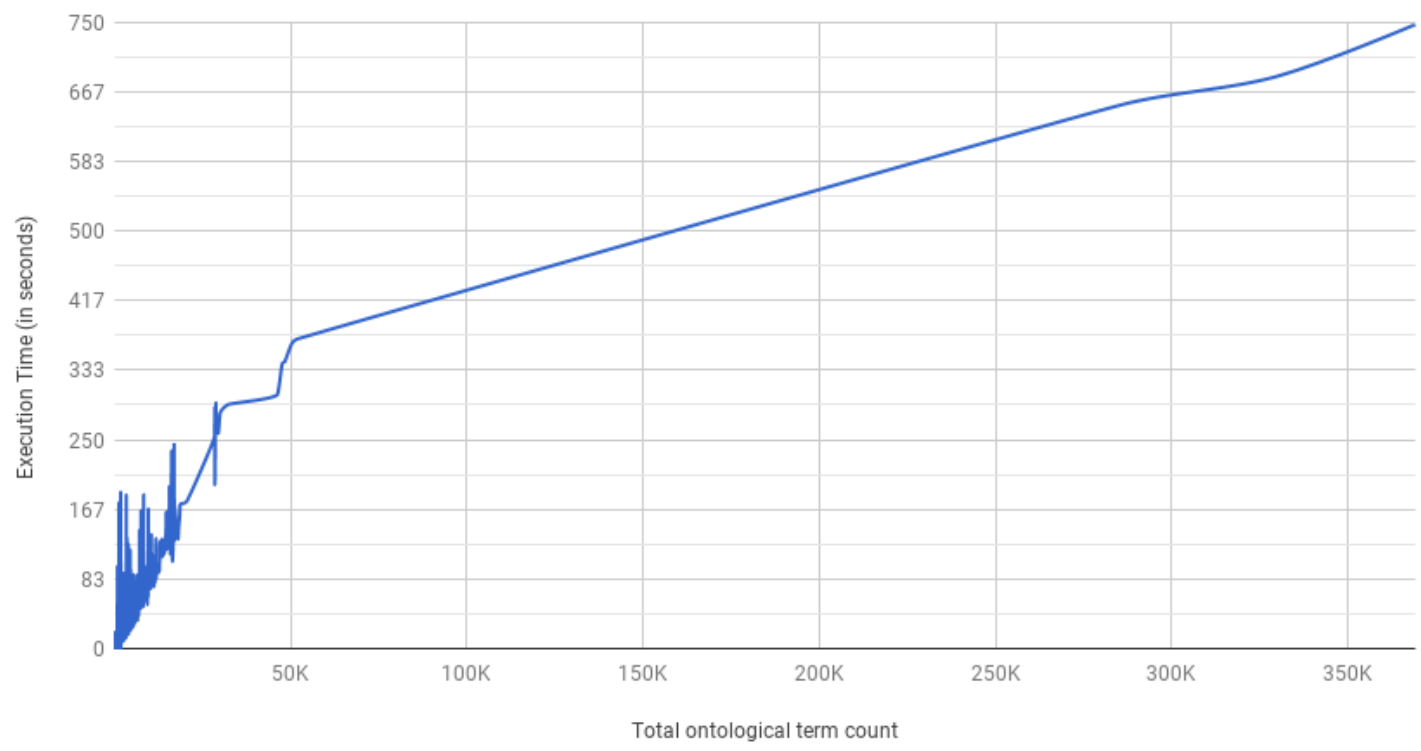

Figure 8.7: Execution time (in seconds) Vs total vocabulary terms

Furthermore, we have calculated how average execution time per vocabulary term vs the total number of vocabulary terms as illustrated in Figure 8.8. As it can be seen from the chart, the time per term gradually decreases when the total number of vocabulary terms increases. This is understandable because the Loupe profiling framework is designed to optimise the execution process by running as much as possible tasks in parallel. Most of the vocabulary terms allow calculation of class-oriented or property-oriented metrics in parallel, thus reduces the effective time per each vocabulary item. When the possibilities of parallel execution increases, it decreases the overall execution time and consequently the average execution time per term.

Similar to the dataset size, we have plotted execution time against the total number of vocabulary term in order to measure the correlation as illustrated in 


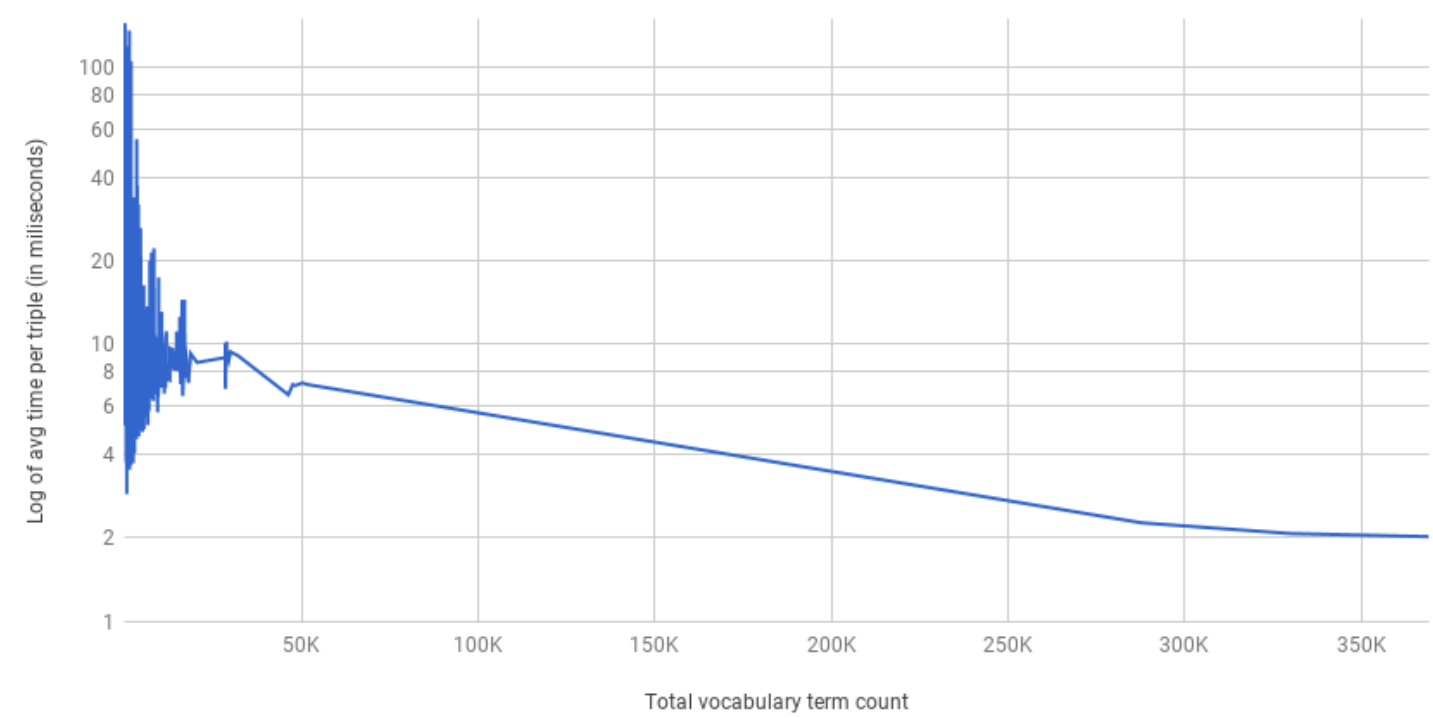

Figure 8.8: Execution time (in seconds) Vs total vocabulary terms

Figure 8.9. There is a positive correlation with a correlation coefficient of 0.74 $(\mathrm{P}<0.0001)$ between the total number of vocabulary terms in the dataset and the execution time. This confirms our intuition that the execution time gradually increases with the number of vocabulary terms.

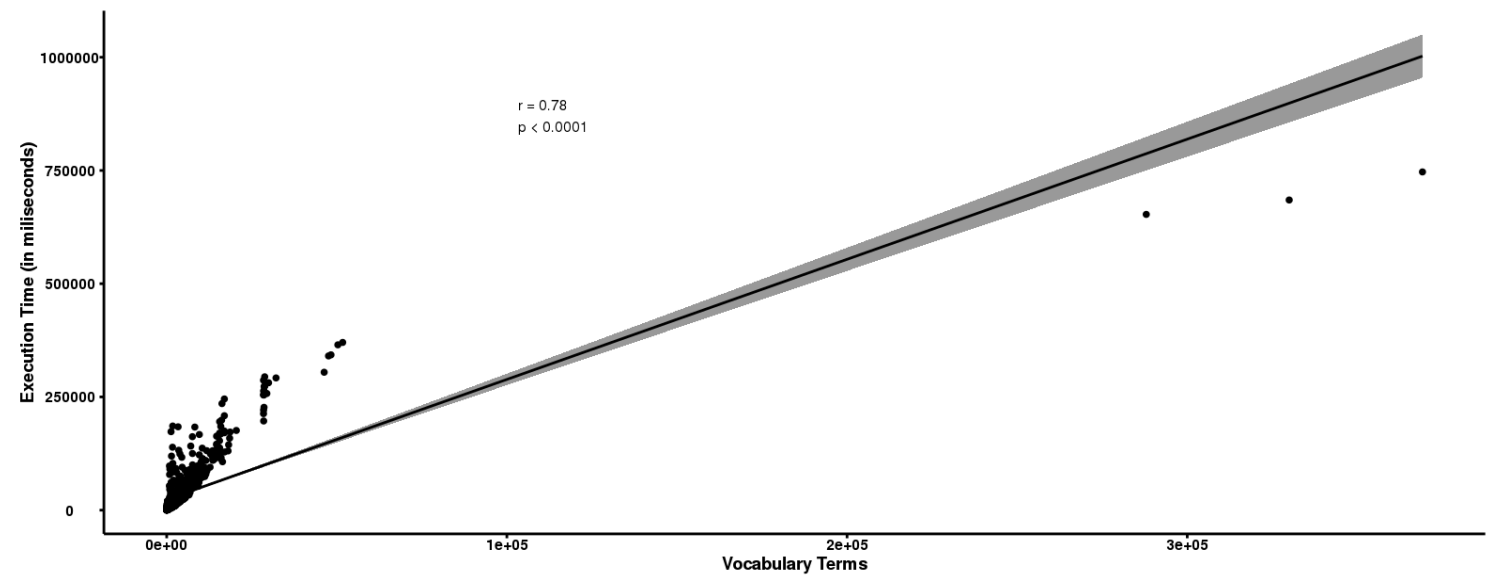

Figure 8.9: Correlation between the total number of vocabulary terms and execution time

Finally, we have examined how the execution time changes with the total number of used classes in the dataset and the total number of used properties in the dataset. 
For this analysis, the number of total classes and properties used in each dataset and their execution time were taken into account. Similar to the previous cases, when there were two or more datasets with the same number of used classes or properties average execution time for all such datasets were calculated. Table 8.13 provides a summary of the distributions of distinct used class counts and used property counts for each of the datasets used in this evaluation.

Table 8.13: Summary of classes used and properties used in each dataset

\begin{tabular}{|l|r|}
\hline Description & Value \\
\hline Number of datasets & 26,063 \\
\hline Distinct used class counts & 191 \\
\hline Minimum used class count & 0 \\
\hline Maximum used class count & 369,144 \\
\hline Median used class count & 139 \\
\hline Average used class count & 8134.06 \\
\hline Distinct used property counts & 984 \\
\hline Minimum used property count & 1 \\
\hline Maximum used property count & 170,583 \\
\hline Median used property count & 1563 \\
\hline Average used property count & 3667.14 \\
\hline
\end{tabular}

Figure 8.10 shows how the execution time varies according to the number of distinct classes in a dataset. On the one hand, as seen from the figure, there is no direct correlation between the number of classes and execution time. On the other hand, Figure 8.11 shows how the execution time varies according to the number of distinct properties in a dataset. As it can be seen from the figure, there is a more direct correlation between the execution time and the number of distinct properties in the dataset.

In conclusion, we have analysed the execution time of the Loupe Linked Data profiling framework with respect to dataset size (in triples) as well as the richness of the vocabulary (measured as the number of distinct classes and properties both as cumulative and separately). Using an experiment, in which, we used execution times of profiling 26 thousand datasets containing 34 billion triples, we have demonstrated that Loupe Linked Data profiling framework scales well with large dataset sets. We have showed that execution time is mostly linearly correlated (i.e., a correlation coefficient closer to 1). While a large majority of datasets had execution of a few seconds, the largest dataset in the experiment with 0.75 billion triples took 13 minutes to complete profiling. 


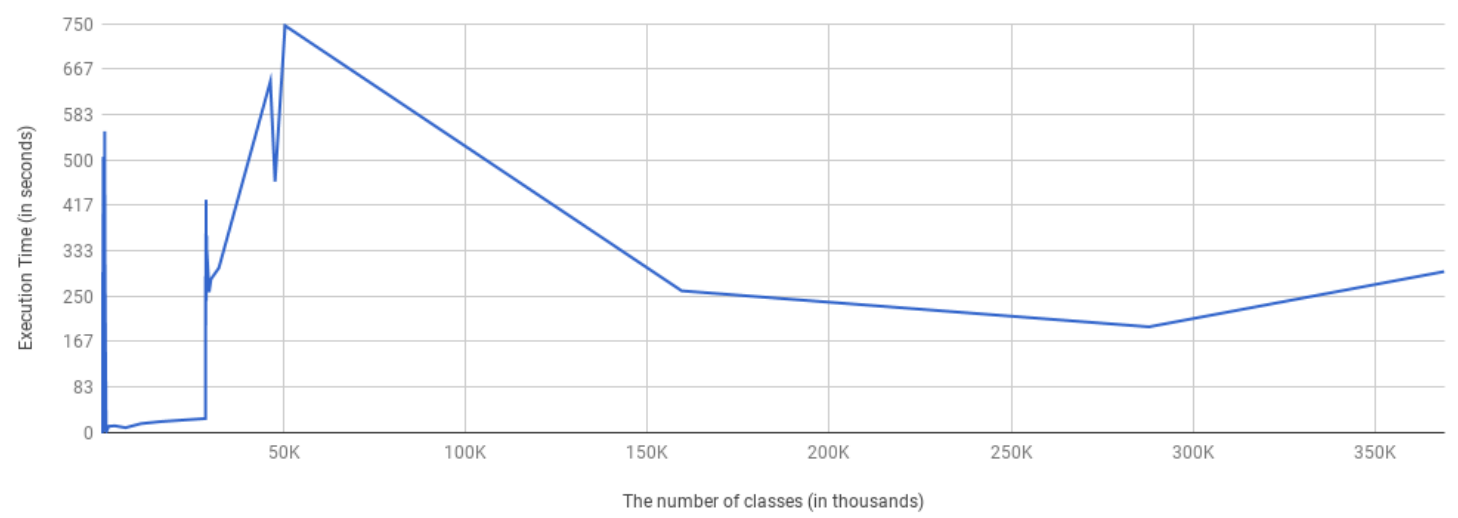

Figure 8.10: Execution time (in seconds) Vs number of classes

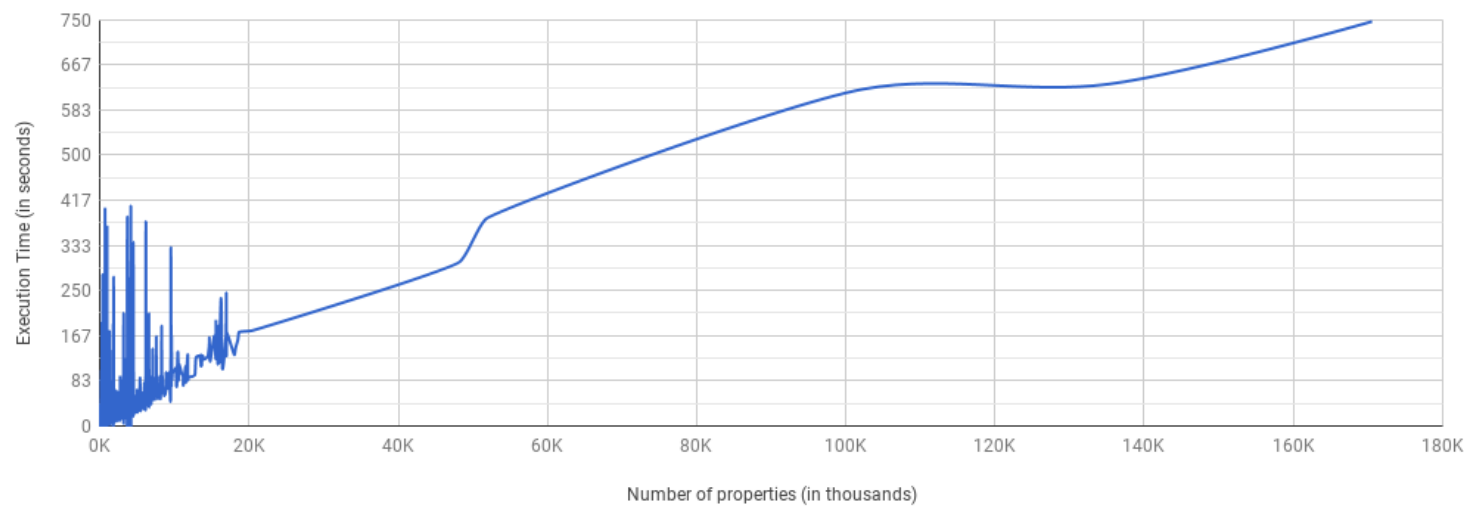

Figure 8.11: Execution time (in seconds) Vs number of properties

\subsection{RDF Shape Induction Method}

The third contribution of the thesis is C3. An automatic RDF shape induction method using the Linked Data profiling metrics. The hypothesis related to C3 is (H5.) that is the proposed automatic RDF Shape Induction method (C3) has a higher accuracy than a baseline approach based on the most frequent value, i.e., a ZeroR based algorithm for generating RDF Shapes with maximum cardinality, minimum cardinality constraints, and range constraints.

Experimental setup: The methodology used for this evaluation is (a) first to create to a gold standard for RDF Shapes by manually annotating the constraints that are considered in the RDF Shape Induction method (i.e., cardinality constraints, and 
range constraints) using the three datasets (English DBpedia ${ }^{10}$, Spanish DBpedia ${ }^{11}$, and 3Cixty [222]) that will be used for the experiments, and then (b) evaluate the performance of the RDF Shape induction method using that manually created gold standard and compare its performance with the baseline.

The proposed RDF Shape induction method was used with different classification algorithms including Random Forrest, Least Square SVM, Neural Network, K-Nearest Neighbour, and Naive Bayes and their performances were recorded. The performance of all those variations were compared against the baseline.

The gold standard: In order to evaluate the performance of the RDF Shape induction, we have created manual annotations for each of the constraints that were considered in the RDF Shape induction method.

For the cardinality constraints, we have selected a set of properties from three different datasets: English DBpedia dataset (version 2016-10), Spanish DBpedia dataset (version 2016-10), and the 3Cixty Nice KB. In order to have diverse set of classes for RDF Shape generation, ten top level classes were selected for DBpedia, namely, dbo:Person, dbo:Place, dbo:Award, dbo:MusicalWork, dbo:Organization, dbo:Film, dbo:Sport, dbo:MeanOfTransportation, dbo:Event, and dbo:Food. For each of these classes, we selected 40 properties and manually annotated their cardinalities. For example, dbo:Person $\rightarrow$ dbo:birthDate is annotated with a minimum cardinality of one and a maximum cardinality of one and dbo:Person $\rightarrow$ dbo:deathDate with minimum cardinality of zero and a maximum cardinality of one. All together, 400 class-property combinations are annotated for each DBpedia dataset. Furthermore, for the 3Cixty Nice dataset we selected ten classes, namely, schema:Person, dul:Place, schema:PostalAddress, ma-ont:Image, schema:Review, linkedevents:Event, linkedevents:YelpBusiness, linkedevents:GooglePlaceBusiness, linkedevents:FacebookBusiness, and sioc:UserAccount. Nevertheless, in 3Cixty dataset, each class had a less number of properties, thus, a total of 215 class-property combinations were manually annotated.

Similarly, for the range constraints, we have annotated 250 class-property combinations from the English and Spanish DBpedia datasets and 215 class-property combinations from the 3Cixty Nice dataset. For example, dbo:Person $\rightarrow$ dbo:birthDate is annotated with the RDF node kind as Literal (as defined in SHACL specification [137]) and the datatype as xsd:Date. dbo:Person $\rightarrow$ dbo:birthPlace is annotated with the node kind as IRI and the class as dbo:Place.

Addressing the class imbalance: From an initial analysis of the dataset, we found that the minimum cardinality value has an imbalance in classifier distribution. For instance, only $23.5 \%$ of DBpedia class-property combinations had MIN1 cardinality

\footnotetext{
${ }^{10}$ https://dbpedia.org/

${ }^{11}$ http://es.dbpedia.org/
} 
class label while $76.5 \%$ had MINO. Similarly, only $20.8 \%$ of 3Cixty class-property combinations had MAX1+ cardinality class label while $79.2 \%$ had MAX1. Because this could affect the classifier performance, we have addressed using sampling techniques. Concretely, we applied SMOTE (Synthetic Minority Over-sampling Technique) [49] for oversampling the rare cardinality class labels. The SMOTE function over-samples rare class labels by using bootstrapping and k-Nearest neighbours to synthetically create additional observations of that class label. In our experiment, we applied an SMOTE over-sampling value of 100 to double the number of positive cases, and an undersampling value of 200 to keep half of what was created as negative cases. It balances the classifier and achieves better performance than only under-sampling the majority class. The class balance before and after applying SMOTE over-sampling are reported in Table 8.14.

Table 8.14: Cardinality class imbalances

\begin{tabular}{|l|l|l|l|l|}
\hline \multirow{2}{*}{ Distribution } & \multicolumn{1}{|c|}{$\begin{array}{c}\text { Minimum } \\
\text { Cardinality }\end{array}$} & \multicolumn{1}{c|}{$\begin{array}{c}\text { Maximum } \\
\text { Cardinality }\end{array}$} \\
\cline { 2 - 5 } & MIN0 & MIN1 & MAX1 & MAX1+ \\
\hline \multicolumn{5}{|c|}{ English DBpedia dataset } \\
\hline Without SMOTE & $76.5 \%$ & $23.5 \%$ & $53 \%$ & $47 \%$ \\
\hline With SMOTE(100,200) & $50 \%$ & $50 \%$ & $50 \%$ & $50 \%$ \\
\hline \multicolumn{5}{|c|}{ Spanish DBpedia dataset } \\
\hline Without SMOTE & $72 \%$ & $28 \%$ & $56 \%$ & $44 \%$ \\
\hline With SMOTE(100,200) & $50 \%$ & $50 \%$ & $50 \%$ & $50 \%$ \\
\hline \multicolumn{5}{|c|}{ 3Cixty Nice dataset } \\
\hline Without SMOTE & $47.2 \%$ & $52.8 \%$ & $79.2 \%$ & $20.8 \%$ \\
\hline With SMOTE(100,200) & $50 \%$ & $50 \%$ & $50 \%$ & $50 \%$ \\
\hline
\end{tabular}

\section{Cardinality Constraints:}

- English DBpedia: Table 8.15 illustrates the performance of generating the minimum cardinality and maximum cardinality constraints the English DBpedia dataset. As it can be seen from the Table, the proposed constraint generation method using the Random Forrest algorithm proven to be effective in achieving an F1 value greater than $90 \%$ for both cases. More concretely, for the minimum cardinality constraints the Random Forest algorithm reached an F1 score value of 97\% with $99 \%$ precision and $96 \%$ recall. Similarly, for the maximum cardinality constraints the Random Forest algorithm achieved an F1 score value of $99 \%$ with $98 \%$ precision and $99 \%$ recall. In comparison, the baseline approach had a lower F1 values with $87 \%$ for the minimum cardinality constraints and $69 \%$ 
Table 8.15: Integrity constraints performance measure for English DBpedia

\begin{tabular}{|c|c|c|c|c|c|c|}
\hline \multirow{2}{*}{$\begin{array}{c}\text { Learning } \\
\text { Algorithm }\end{array}$} & \multicolumn{2}{|c|}{ Minimum Cardinality } & \multicolumn{2}{c|}{ Maximum Cardinality } \\
\cline { 2 - 7 } & Precision & Recall & F1 & Precision & Recall & F1 \\
\hline Random Forest & $\mathbf{0 . 9 9}$ & $\mathbf{0 . 9 6}$ & $\mathbf{0 . 9 7}$ & $\mathbf{0 . 9 8}$ & $\mathbf{0 . 9 9}$ & $\mathbf{0 . 9 9}$ \\
\hline Least Squares SVM & 0.99 & 0.94 & 0.97 & 0.85 & 0.96 & 0.90 \\
\hline Neural Network & 0.97 & 0.95 & 0.96 & 0.82 & 0.96 & 0.88 \\
\hline K-Nearest Neighbour & 0.95 & 0.93 & 0.94 & 0.88 & 0.87 & 0.87 \\
\hline Naive Bayes & 0.94 & 0.84 & 0.88 & 0.91 & 0.77 & 0.83 \\
\hline Baseline & $\mathbf{0 . 7 6}$ & $\mathbf{1 . 0}$ & $\mathbf{0 . 8 7}$ & $\mathbf{0 . 5 3}$ & $\mathbf{1 . 0 0}$ & $\mathbf{0 . 6 9}$ \\
\hline
\end{tabular}

for the maximum cardinality constraints. It is important to note that because the ZeroR algorithm used in the baseline always predicts the majority class, it has a recall of $100 \%$ but the precision is lower than the RDF Shape Induction method using Linked Data profiling metrics as inputs. Furthermore, we can see that when the RDF Shape induction method is used with other classification algorithms such as Least Square SVM, Neural Network, K-Nearest Neighbour, and Naive Bayes they also yielded significantly high F1 values.

- Spanish DBpedia: Table 8.16 illustrates the performance of generating the minimum cardinality and maximum cardinality constraints the Spanish DBpedia dataset. Similar to the English DBpedia dataset, the proposed constraint generation method using the Random Forrest algorithm achieved the best results. More concretely, for the minimum cardinality constraints the Random Forest algorithm reached an F1 score value of $90 \%$ with $85 \%$ precision and $87 \%$ recall. Similarly, for the maximum cardinality constraints the Random Forest algorithm achieved an F1 score value of $93 \%$ with $92 \%$ precision and $93 \%$ recall. In comparison, the baseline approach had a lower F1 values with $83 \%$ for the minimum cardinality constraints and $72 \%$ for the maximum cardinality constraints. The other algorithms variations that were used with the RDF Shape induction method, i.e., Least Square SVM, Neural Network, K-Nearest Neighbour, and Naive Bayes also achieved better results than the baseline. In comparison with the English DBpedia dataset, the performance for the cardinality constraint generation was a bit lower for the Spanish DBpedia dataset.

- 3cixty Nice Table 8.17 illustrates the performance of generating the minimum cardinality and maximum cardinality constraints the 3Cixty Nice dataset. Similar to the previous two datasets, the proposed constraint generation method using the Random Forrest algorithm was proven to be the most effective with an F1 value greater than $90 \%$ for both cases. More concretely, for the minimum 
Table 8.16: Cardinality constraints performance measure for Spanish DBpedia

\begin{tabular}{|c|c|c|c|c|c|c|}
\hline \multirow{2}{*}{$\begin{array}{c}\text { Learning } \\
\text { Algorithm }\end{array}$} & \multicolumn{2}{|c|}{ Minimum Cardinality } & \multicolumn{3}{c|}{ Maximum Cardinality } \\
\cline { 2 - 7 } & Precision & Recall & F1 & Precision & Recall & F1 \\
\hline Random Forest & $\mathbf{0 . 9 0}$ & $\mathbf{0 . 8 5}$ & $\mathbf{0 . 8 8}$ & $\mathbf{0 . 9 2}$ & $\mathbf{0 . 9 3}$ & $\mathbf{0 . 9 3}$ \\
\hline Least Squares SVM & 0.85 & 0.89 & 0.87 & 0.81 & 0.88 & 0.84 \\
\hline Neural Network & 0.87 & 0.82 & 0.84 & 0.89 & 0.85 & 0.87 \\
\hline K-Nearest Neighbour & 0.84 & 0.82 & 0.83 & 0.82 & 0.79 & 0.80 \\
\hline Naive Bayes & 0.71 & 0.73 & 0.72 & 0.79 & 0.80 & 0.79 \\
\hline Baseline & $\mathbf{0 . 7 2}$ & $\mathbf{1 . 0 0}$ & $\mathbf{0 . 8 4}$ & $\mathbf{0 . 5 6}$ & $\mathbf{1 . 0 0}$ & $\mathbf{0 . 7 2}$ \\
\hline
\end{tabular}

Table 8.17: Cardinality constraints performance measures for 3cixty

\begin{tabular}{|c|c|c|c|c|c|c|}
\hline \multirow{2}{*}{$\begin{array}{c}\text { Learning } \\
\text { Algorithm }\end{array}$} & \multicolumn{2}{|c|}{ Minimum Cardinality } & \multicolumn{2}{c|}{ Maximum Cardinality } \\
\cline { 2 - 7 } & Precision & Recall & F1 & Precision & Recall & F1 \\
\hline Random Forest & $\mathbf{0 . 9 6}$ & $\mathbf{0 . 8 7}$ & $\mathbf{0 . 9 2}$ & $\mathbf{0 . 8 9}$ & $\mathbf{0 . 9 4}$ & $\mathbf{0 . 9 2}$ \\
\hline Neural Network & 0.88 & 0.88 & 0.81 & 0.81 & 0.83 & 0.82 \\
\hline Least Squares SVM & 0.77 & 0.73 & 0.75 & 0.81 & 0.88 & 0.84 \\
\hline Naive Bayes & 0.72 & 0.69 & 0.70 & 0.73 & 0.83 & 0.77 \\
\hline K-Nearest Neighbour & 0.70 & 0.67 & 0.68 & 0.70 & 0.83 & 0.76 \\
\hline Baseline & $\mathbf{0 . 5 3}$ & $\mathbf{1 . 0 0}$ & $\mathbf{0 . 6 9}$ & $\mathbf{0 . 7 9}$ & $\mathbf{1 . 0 0}$ & $\mathbf{0 . 8 8}$ \\
\hline
\end{tabular}

cardinality constraints the Random Forest algorithm reached an F1 score value of $92 \%$ with $96 \%$ precision and $87 \%$ recall. Similarly, for the maximum cardinality constraints the Random Forest algorithm achieved an F1 score value of $92 \%$ with $89 \%$ precision and $94 \%$ recall. In comparison, the baseline approach had a lower F1 values with $69 \%$ for the minimum cardinality constraints and $88 \%$ for the maximum cardinality constraints. Nevertheless, in this case, the Naive Bayes algorithm had a performance lower than the baseline for the minimum cardinality constraints. For maximum cardinality constraints, all other classification algorithms except for the Random Forest algorithm had lower performances.

Discussion: The goal of the aforementioned experiments was to measure the performance of minimum and maximum cardinality constraint generation using the RDF Shape induction method with the Linked Data profiling metrics as input features and compare the performance with the baseline. The prediction performance of cardinality constraint classifiers was measured by precision, recall and F1 score. In our experimental analysis, we have used ZeroR classifier as our baseline. It is a simple classifier that predicts a majority class as the prediction and can be used to provide a baseline for the prediction tasks. For the baseline, we used the data 
before oversampling with the SMOTE algorithm. Overall, the RDF Shape induction method using the Random Forest algorithm achieved the best results for all three datasets. Furthermore, neural network and least squares SVM also achieved high F1 scores greater than $90 \%$ for the English DBpedia Dataset. In all three datasets for both minimum cardinality and maximum cardinality, our approach with the Random Forrest classification algorithm achieved better results than the baseline approach.

\section{Range Constraints:}

English DBpedia: Table 8.18 illustrates the performance of generating the range constraints, more specifically, the object node type constraints for the English DBpedia dataset. Similar to the previous experiments on the cardinality constraints, the proposed constraint generation method using the Random Forrest algorithm achieved the best results reaching an F1 value of $95 \%$ with a precision of $94 \%$ and a recall of $95 \%$. This is significantly higher than the baseline performance; an F1 value of $76 \%$ with a precision of $62 \%$ and a recall of $100 \%$. It is important to note that because the Zero $R$ algorithm used in the baseline always predicts the majority class, it reaches a recall of $100 \%$ but both the precision and F1 value are lower than the RDF Shape Induction method using Linked Data profiling metrics as inputs. The other algorithm variations used with the proposed RDF Shape induction method such as Least Square SVM, Neural Network, K-Nearest Neighbour, and Naive Bayes also achieve better F1 values than the baseline approach.

Table 8.18: Range constraints performance measure for the English DBpedia dataset.

\begin{tabular}{|l|r|r|r|}
\hline Learning Algorithm & Precision & Recall & F1 \\
\hline Random Forest & $\mathbf{0 . 9 4 5 7}$ & $\mathbf{0 . 9 5 2 7}$ & $\mathbf{0 . 9 5 9 4}$ \\
\hline Least Squares SVM & 0.8596 & 0.9231 & 0.8902 \\
\hline Multilayer Perceptron & 0.8262 & 0.8657 & 0.8456 \\
\hline K-Nearest Neighbour & 0.8361 & 0.8425 & 0.8393 \\
\hline Naive Bayes & 0.8953 & 0.7951 & 0.8422 \\
\hline Baseline & $\mathbf{0 . 6 1 7 4}$ & $\mathbf{1 . 0}$ & $\mathbf{0 . 7 6 3 4}$ \\
\hline
\end{tabular}

Spanish DBpedia: Table 8.19 illustrates the performance of generating the object node type range constraints for the Spanish DBpedia dataset. Similar to the English DBpedia dataset, the proposed constraint generation method using the Random Forrest algorithm achieved the best results reaching an F1 value of $88 \%$ with a precision of $87 \%$ and a recall of $89 \%$. This is significantly higher than the baseline performance; an $\mathrm{F} 1$ value of $68 \%$ with a precision of $52 \%$ and a recall of $100 \%$. The performance for the Spanish DBpedia dataset is a bit less than the performance for the English DBpedia dataset in all algorithm variations used in the experiment. 
Table 8.19: Range constraints performance measure for the Spanish DBpedia dataset.

\begin{tabular}{|l|r|r|r|}
\hline Learning Algorithm & Precision & Recall & F1 \\
\hline Random Forest & $\mathbf{0 . 8 7 4 1}$ & $\mathbf{0 . 8 9 5 4}$ & $\mathbf{0 . 8 8 4 6}$ \\
\hline Least Squares SVM & 0.8348 & 0.8416 & 0.8381 \\
\hline Multilayer Perceptron & 0.7942 & 0.7701 & 0.7819 \\
\hline K-Nearest Neighbour & 0.7714 & 0.7808 & 0.7761 \\
\hline Naive Bayes & 0.7620 & 0.7901 & 0.7758 \\
\hline Baseline & $\mathbf{0 . 5 2 0 0}$ & $\mathbf{1 . 0 0}$ & $\mathbf{0 . 6 8 4 2}$ \\
\hline
\end{tabular}

3Cixty Nice dataset: Table 8.20 illustrates the performance of generating the object node type range constraints for the 3Cixty Nice dataset. Similar to the previous two cases of the English DBpedia dataset and the Spanish DBpedia dataset, the proposed constraint generation method using the Random Forrest algorithm achieved the best results reaching an $\mathrm{F} 1$ value of $92 \%$ with a precision of $93 \%$ and a recall of $92 \%$. This is significantly higher than the baseline F1 value of $77 \%$ with a precision of $63 \%$ and a recall of $100 \%$. All the other algorithm variations, i.e., Least Square SVM, Neural Network, K-Nearest Neighbour, and Naive Bayes also have higher F1 values than the baseline.

Table 8.20: Range constraints performance for the 3Cixty dataset.

\begin{tabular}{|l|r|r|r|}
\hline Learning Algorithm & Precision & Recall & F1 \\
\hline Random Forest & $\mathbf{0 . 9 3 3 3}$ & $\mathbf{0 . 9 0 3 2}$ & $\mathbf{0 . 9 1 8 0}$ \\
\hline Least Squares SVM & 0.9375 & 0.8823 & 0.9091 \\
\hline Multilayer Perceptron & 0.8148 & 0.9167 & 0.8627 \\
\hline K-Nearest Neighbour & 0.8266 & 0.7462 & 0.8275 \\
\hline Naive Bayes & 0.7837 & 0.8285 & 0.8055 \\
\hline Baseline & $\mathbf{0 . 6 3 7 2}$ & $\mathbf{1 . 0 0}$ & $\mathbf{0 . 7 7 8 4}$ \\
\hline
\end{tabular}

\subsection{Methods for Quality Assessment and Repair}

The fourth contribution of the thesis is C4. A set of methods for automatic quality assessment and repair using Linked Data profiling metrics and RDF Shapes.

The methodology used for this evaluation is to select a set of use cases related to quality assessment and repair in Linked Data and develop methods to address those use cases using the Linked Data profiling information and RDF Shapes. In the following sections, we evaluate more concrete hypotheses related to each of the methods purposed in this thesis. 


\subsubsection{Prediction of Inconsistent Mappings}

The first method purposed under $C_{4}$ is $C_{4} .1$ a method to predict inconsistent mappings based on Linked Data profiling metrics.

Use case: As discussed in Chapter 1, many Linked Data sources use RDF mappings in the RDF generation process to transform non-RDF source data into Linked Data represented as RDF. Nevertheless, mappings can contain errors and can be inconsistent. Because large-scale datasets, such as DBpedia, have a large number of mappings to generate data, it is not feasible to inspect all such mappings manually to ensure their consistency. Thus, the proposed technique detects inconsistent mappings automatically in a data-driven manner by analysing profiling information from instance data extracted using the Linked Data profiling framework.

The hypothesis related to this method is that (H6.) the proposed method to predict inconsistent mappings based on Linked Data profiling metrics (C4.1) can achieve an accuracy of $80 \%$ in identifying inconsistent mappings..

Metrics: The goal of the proposed technique is to identify inconsistent mappings from a set of RDF generation mappings, which are used for transforming non-RDF data sources into RDF. Thus, to measure the performance of the proposed approach, we have used the accuracy of the classification between inconsistent and consistent mappings.

Dataset: For the evaluation of the proposed method, we have used data from several DBpedia multilingual datasets from the version 2016-10 that was released on July, 2017. Concretely, English DBpedia (EN), Spanish DBpedia (ES), Dutch DBpedia (NL), and Greek DBpedia (GR) were used in the experiments. In addition, all RDF mappings related to the generation of these datasets were extracted.

The RDF data from each dataset could be used directly in the Linked Data Profiling Framework. Nevertheless, the triples in each dataset did not contain information for extracting provenance information about each individual triple, i.e., which were the infobox and the mapping that were used for generating the triple.

Because we needed this information to identify the inconsistent mappings, we have used the n-quad files which contained this information encoded as query parameters in an IRI and extracted this information. Listing 8.1 illustrates an example n-quad from the quad-turtle (.tql) files available in each DBpedia release. This example is about the triple, "dbr:Madrid dbo:country dbr:Spain" and the fourth element in the n-quad provides provenance information about the triple. For instance, it specifies that the infobox "Ficha_de_localidad_de_Espana" and the infobox attribute "maps_loc" were used to extract the data for generating the triple. 


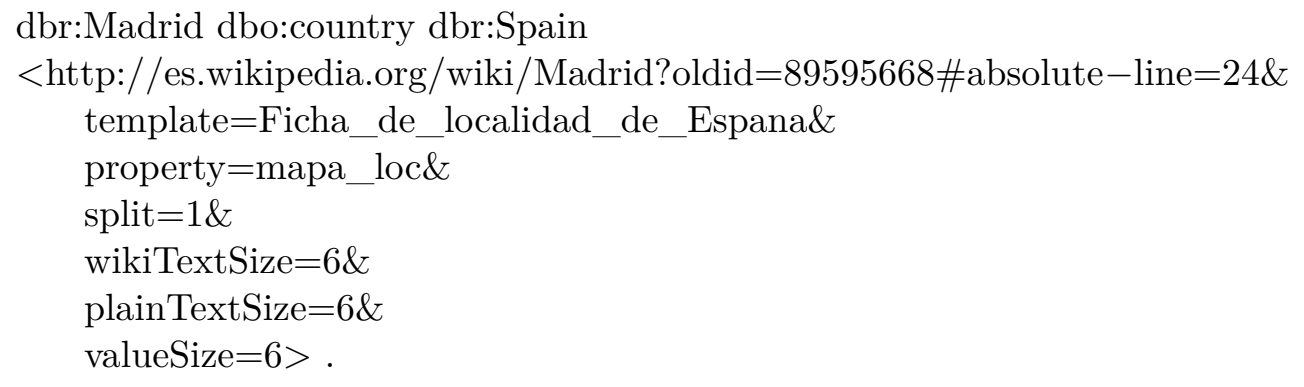

Listing 8.1: Example of an n-quad (from .tql file available in each DBpedia release)

In order to use this information with the Linked Data profiling framework and generate the metrics required by the proposed approach, we used a "reification" process to extract the provenance information encoded as query parameters in IRIs and represent them explicitly in RDF. First, we used regular expressions to extract the values of query parameters template and property for each of the triples. Then, we represented that information in RDF as illustrated in Listing 8.2.

_.uuid rdf:type rdf:Statement ; rdf:subject dbo:Madrid ; rdf:predicate dbo:country ; dbp:template "Ficha_de_localidad_de_Espana"; dbp:attribute "mapa_loc" ; prov:wasDerivedFrom $<$ http://es.wikipedia.org/wiki/Madrid?oldid=89595668 \#absolute-line $=24>$.

Listing 8.2: An example reification result

Experimental setup: We have designed the experiment to evaluate the hypothesis that inconsistent mappings used for RDF generation can be identified with high accuracy than a baseline approach using machine learning techniques using Linked Data profiling information as features. The methodology followed is to first create a gold standard by manually annotating a set of mappings by human experts and then use that gold standard for estimating the classification accuracy of the proposed method and the baseline approach for validating the hypothesis.

DBpedia provides triples with IRI objects (i.e., object properties) and literal objects (i.e., datatype properties) separately in two different files. Thus, in order to have more fine-grained insights about inconsistent mappings, we performed the analysis separately for triples with IRI objects and literal objects. In order annotate inconsistent mappings, we need to generate mapping pairs that generate triples with distinct properties (e.g., dbo:height and dbo:elevation) but with the same subject (e.g., dbr:Mount_Everest) and object (e.g., 8848) pairs. For this, we have annotated 
mapping pairs for four language combinations: English-Spanish (EN-ES), SpanishGerman (ES-DE), English-Dutch (EN-NL), English-Greek (EN-GR). The language pairs were selected based on the availability of multilingual experts who could annotate if the mappings were consistent or not. In total, 758 mapping pairs were annotated to be used as the gold standard. Each annotation was done by a multilingual expert who had good knowledge of both languages of the language pair (e.g., English and Spanish) and was verified by another expert with similar knowledge. When there was no inter-annotator agreement, the mapping pair was discussed among all the experts and agreement was made. Ten multilingual experts from different language chapters of DBpedia participated in the creation of the gold standard.

The gold standard was used to calculate the accuracy of the classification of inconsistent mappings using different approaches.

\section{Results:}

In this section, we present the classification accuracy results for different scenarios. First, we explain the results for mapping pairs of English-Spanish DBpedia datasets. As discussed before, we present the results separately for mappings that generate triples with literal objects such as strings, numerics, and dates and for mappings that generate triples with IRI objects.

English-Spanish literal mappings: For this setting, 240 mapping pairs have been manually annotated by contributors from experts from the DBpedia community, fluent in both languages. We reserved 14 annotations (holdout set) for the revalidation of the model. Then, we trained a predictive model with the remaining 226 annotations. The data file ${ }^{12}$ is publicly available, which also contains a statistic summary for each feature in the dataset. The training set contains 182 mappings annotated as "Correct" and 44 as "Incorrect". Therefore, the baseline classifier, which always assigns the most frequent class for every instance, has an accuracy of $64.29 \%$. This classifier establishes the baseline accuracy that is to be compared with the accuracy of the proposed model to validate the hypothesis related to this method. As it is shown in Table 8.22, with the Random Forest classification algorithm, the proposed method archives a classification accuracy of $94.25 \%$.

English-Spanish IRI mappings: For this setting, we have used 80 mapping pairs that generate triples with IRI objects. The data file ${ }^{13}$ is publicly available, which also contains a statistic summary for each feature in the dataset. The training set contains 80 mappings in total, out of which 71 mappings are annotated as "Correct" and 9 as "Incorrect". Therefore, the baseline classifier, which always assigns the most frequent class for every instance, has an accuracy of $88.75 \%$.

\footnotetext{
${ }^{12}$ https://wWw. openml.org/d/41120

${ }^{13}$ https : //WWw. openml .org/d/41121
} 
Table 8.21: English-Spanish literals results

\begin{tabular}{|l|c|c|c|}
\hline & $\begin{array}{l}\text { Random } \\
\text { Forest }\end{array}$ & $\begin{array}{l}\text { Multilayer } \\
\text { Perceptron }\end{array}$ & SMO \\
\hline Correctly Classified & 211 & 213 & 211 \\
\hline Incorrectly Classified & $15(6.64 \%)$ & $13(5.75 \%)$ & $15(6.64 \%)$ \\
\hline Classifier accuracy & $93.36 \%$ & $\mathbf{9 4 . 2 5 \%}$ & $93.36 \%$ \\
\hline Baseline & \multicolumn{3}{|c|}{$\mathbf{6 4 . 2 9 \%}$} \\
\hline Kappa statistic & 0.7865 & 0.8117 & 0.7748 \\
\hline Mean absolute error & 0.1101 & 0.0641 & 0.0664 \\
\hline Root mean squared error & 0.2288 & 0.2276 & 0.2576 \\
\hline Relative absolute error & $34.8987 \%$ & $20.3324 \%$ & $21.0402 \%$ \\
\hline Root relative squared error & $57.7554 \%$ & $57.4747 \%$ & $65.0442 \%$ \\
\hline Total Number of Instances & 226 & 226 & 226 \\
\hline
\end{tabular}

Prediction of inconsistent mappings on object properties If we apply this predictive model to object properties, i.e., objects being IRIs, the accuracy (correctly classified instances) is $95.00 \%$, very similar to the $93.36 \%$ achieved when objects are literals. Notice that this IRIs dataset, or any other IRI dataset, has not been "seen" by the model in its training.

The dataset (80 instances, 71 Correct and 9 Incorrect) is publicly available ${ }^{14}$.

\section{Prediction on datasets in other languages}

Since creating a gold standard for each language is a costly approach, it would be desirable to have a universal model (trained with data annotated in any language) capable of predicting incorrect mappings not only in the language it has been trained on, but in other languages. This would be some sort of multilingual predictor. Notice that the manual annotation of each dataset is a highly-specialised task that requires humans with excellent knowledge of the two languages involved. A unique model would save a lot of human work.

We have applied this specific predictive model (EN-ES-lit) to the dataset ESDE-IRI containing 110 annotations. The accuracy (correctly classified instances) is $87.28 \%$, very similar to the $85.71 \%$ achieved for EN-ES-IRIs, but not as good as the EN-ES-lit with $93.36 \%$.

However, the English-Dutch literals (EN-NL-lit), with 83 annotations (35 Incorrect, 48 Correct) has an accuracy of only $61.45 \%$ If we create a predictive model for this data we get an accuracy of $71.08 \%$.

\footnotetext{
${ }^{14}$ https : //WwW. openml . org/d/40744
} 
Table 8.22: Summary of classifiers output.

\begin{tabular}{|l|c|c|c|}
\hline & Random Forest & Multilayer Perceptron & SMO \\
\hline Correctly Classified Instances & 211 & 213 & 211 \\
\hline Incorrectly Classified Instances & $15(6.64 \%)$ & $13(5.75 \%)$ & $15(6.64 \%)$ \\
\hline Classifier accuracy & $93.36 \%$ & $\mathbf{9 4 . 2 5 \%}$ & $93.36 \%$ \\
\hline Baseline & \multicolumn{3}{|c|}{$\mathbf{8 8 . 7 5 \%}$} \\
\hline Kappa statistic & 0.7865 & 0.8117 & 0.7748 \\
\hline Mean absolute error & 0.1101 & 0.0641 & 0.0664 \\
\hline Root mean squared error & 0.2288 & 0.2276 & 0.2576 \\
\hline Relative absolute error & $34.8987 \%$ & $20.3324 \%$ & $21.0402 \%$ \\
\hline Root relative squared error & $57.7554 \%$ & $57.4747 \%$ & $65.0442 \%$ \\
\hline Total Number of Instances & 226 & 226 & 226 \\
\hline
\end{tabular}

Table 8.23: Summary of the results of applying the predictive model (EN-ES literals) to the IRIs dataset

\begin{tabular}{ll}
\hline Correctly Classified Instances & $12(85.71 \%)$ \\
Incorrectly Classified Instances & $2(14.29 \%)$ \\
Kappa statistic & 0.6889 \\
Mean absolute error & 0.2479 \\
Root mean squared error & 0.3102 \\
Total Number of Instances & 14 \\
\hline
\end{tabular}

For Dutch (EN-NL-IRIs), with 28 annotations (19 Incorrect, 9 Correct) has an accuracy very low as well, only $67.86 \%$. A predictive model with this dataset would get an accuracy of $100 \%$. A detailed analysis, changing the aleatory seed, produces accuracy values around $94 \%$. This dependency on the seed indicates that the number of instances is too low.

For EN-GR-lit, with 64 annotations (30 Incorrect, 33 Correct) we get an accuracy of $77.78 \%$. If we create a predictive model for this data we get an accuracy of $73.02 \%$. All this information is condensed in Table 8.25, where we can see that for the 4 language-pairs studied the model for IRIs is always better than the model for literals. Besides, we can see that the EN-ES-lit model is good predicting incorrect mappings for some language-pairs (such as ES-DE-IRI or EN-GR-IRI), but not so good for another language-pairs (such as EN-NL-lit or EN-NL-IRI). Our conclusion is that it is not feasible to have a unique predictive model for all the language-pairs.

Optimising the model We have computed a Principal Component Analysis over the EN-ES-lit dataset. This analysis produces a new set of attributes, a linear 
Table 8.24: Detailed accuracy data and confusion matrix for the predictive model (EN-ES literals) on the IRIs dataset

\begin{tabular}{|c|c|c|c|c|c|c|c|c|c|}
\hline \multirow[t]{3}{*}{ Accuracy } & TP Rate & FP Rate & Precision & Recall & F-Measure & $\mathrm{MCC}$ & ROC Area & PRC Area & Class \\
\hline & 0.800 & 0.111 & 0.800 & 0.800 & 0.800 & 0.689 & 0.978 & 0.967 & Incorrect \\
\hline & 0.889 & 0.200 & 0.889 & 0.889 & 0.889 & 0.689 & 0.978 & 0.989 & Correct \\
\hline Avg. & 0.857 & 0.168 & 0.857 & 0.857 & 0.857 & 0.689 & 0.978 & 0.981 & \\
\hline \multicolumn{10}{|l|}{$\begin{array}{l}\text { Confusion } \\
\text { ma- } \\
\text { trix }\end{array}$} \\
\hline & & $\begin{array}{l}\text { Pred. } \\
\text { Correct }\end{array}$ & $\begin{array}{l}\text { Pred. } \\
\text { Incorrect }\end{array}$ & & & & & & \\
\hline \multicolumn{2}{|c|}{ True Correct } & 8 & 1 & & & & & & \\
\hline \multicolumn{2}{|c|}{ True Incorrect } & 1 & 4 & & & & & & \\
\hline
\end{tabular}

Table 8.25: Summary of the prediction accuracy for other language-pairs

\begin{tabular}{|c|c|c|c|c|c|c|c|c|c|}
\hline & \multicolumn{2}{|c|}{ EN-ES } & \multicolumn{2}{|c|}{ ES-DE } & \multicolumn{2}{|c|}{ EN-NL } & \multicolumn{2}{|c|}{ EN-GR } \\
\hline & & lit & IRI & lit & IRI & lit & IRI & lit & IRI \\
\hline \multirow{2}{*}{ Accuracy } & Trained model & \multirow{2}{*}{$93.36 \%$} & $95.00 \%$ & 93.91 & $96.36 \%$ & $71.08 \%$ & $\sim 94 \%$ & $73.02 \%$ & $89.71 \%$ \\
\hline & En-ES-lit model & & $65.00 \%$ & $88.70 \%$ & $87.28 \%$ & $61.45 \%$ & $67.86 \%$ & $77.78 \%$ & $88.24 \%$ \\
\hline \multicolumn{2}{|l|}{ Baseline } & $82.94 \%$ & $88.75 \%$ & 87.83 & $92.72 \%$ & $57.83 \%$ & $32.14 \%$ & $52.38 \%$ & $64.71 \%$ \\
\hline \multirow{3}{*}{ Annotations } & Total instances & 211 & 80 & 115 & 110 & 83 & 28 & 63 & 68 \\
\hline & 'Correct' instances & 175 & 71 & 101 & 102 & 35 & 9 & 33 & 44 \\
\hline & 'Incorrect' instances & 36 & 9 & 14 & 8 & 48 & 19 & 30 & 24 \\
\hline
\end{tabular}

combination of the initial attributes, ranked by their contribution to the data variance, from more relevant to less relevant. Specifically, keeping a variance of $95 \%$, we can move from a dataset with 23 attributes to a dataset with 13 attributes (12 numeric attributes and one nominal attribute). If we compute a new model using again Random Forest we achieve an accuracy of $92.48 \%$, very close to the $93.36 \%$ achieved with all the attributes. Table 8.26 shows the effect of the progressive elimination of less relevant attributes on the accuracy of the predictive model. The 'Order' column is the variable ID, from lowest to highest variance. For instance, the first row in this table shows that the effect of removing the first lowest variance PCA attribute, having a 0.55 variance (indeed std. dev.), produces a predictive model with accuracy $93.81 \%$. The second row shows that the effect of removing the two lowest variance PCA attributes, in which the second attribute has a variance (std. dev.) of 0.688, produces a predictive model with accuracy $93.81 \%$. In this table we can see that the effect of removing the three lowest variance PCT attributes is the same: a model accuracy of $93.81 \%$. However, removing the four lowest we get lower accuracy $(93.36 \%)$, that maintains this value when removing the $5^{\text {th }}$ and $6^{\text {th }}$ lowest variance PCA attributes. But, when we remove the $8^{\text {th }}$ we get an increment in the accuracy. If we remove more PCA attributes we get lower values. The last row shows the effect of removing all the PCA attributes except the one with the highest variance. Using 
Table 8.26: Principal Components Analysis (PCA): Effect of reducing the number of PCA attributes on the accuracy of the predictive model

\begin{tabular}{cllll}
\hline \multirow{2}{*}{ Order } & \multicolumn{4}{c}{ Data variance } \\
\cline { 2 - 5 } & \multicolumn{3}{c}{$95 \%$} & \multicolumn{2}{c}{$90 \%$} \\
\cline { 2 - 5 } & Std.dev. & Accuracy & Std. dev. & Accuracy \\
\cline { 2 - 5 } 1 & 0.55 & $93.8053 \%$ & 0.779 & $93.8053 \%$ \\
2 & 0.688 & $93.8053 \%$ & 0.91 & $93.3628 \%$ \\
3 & 0.779 & $93.8053 \%$ & 0.938 & $93.3628 \%$ \\
4 & 0.91 & $93.3628 \%$ & 0.996 & $93.3628 \%$ \\
5 & 0.938 & $93.3628 \%$ & 1.047 & $92.9204 \%$ \\
6 & 0.996 & $93.3628 \%$ & 1.074 & $\mathbf{9 3 . 8 0 5 3 \%}$ \\
7 & 1.047 & $92.9204 \%$ & 1.207 & $92.9204 \%$ \\
8 & 1.074 & $\mathbf{9 3 . 8 0 5 3 \%}$ & 1.277 & $88.4956 \%$ \\
9 & 1.207 & $92.9204 \%$ & 1.939 & $80.9735 \%$ \\
10 & 1.277 & $88.4956 \%$ & & \\
11 & 1.939 & $80.9735 \%$ & & \\
\hline
\end{tabular}

this unique attribute we get a model with accuracy of $80.97 \%$.

It is remarkable that with a reduction from 23 to 4 attributes, the Random Forest classifier obtains a slightly better accuracy (93.81\%) than the initial model with 23 attributes (accuracy 93.36\%).

If we repeat the PCA keeping a variance slightly lower than before, specifically to 90\%, we get now ten PCA attributes. In Table 8.26, we can see that we can remove the six attributes with lowest variance while getting a predictive model with accuracy of $93.81 \%$. Again, we get a model with only four attributes that produces a predictive model with accuracy of $93.81 \%$.

Figure 8.12 shows a 2D projection of the 4D PCA space after the optimization. Although there is no clear separation for the classes, despite the $93 \%$ accuracy of the model, we can see a clear concentration (cluster) for Incorrect instances (dashed black ellipse). This concentration could provide the separation between classes required for such a good classification.

\subsubsection{Noise Detection in Information Extraction}

The second method proposed under the contribution $C_{4}$ is $\mathrm{C} 4.2$ a noise detection method for information extraction tools based on Linked Data profiling metrics and RDF Shapes. 


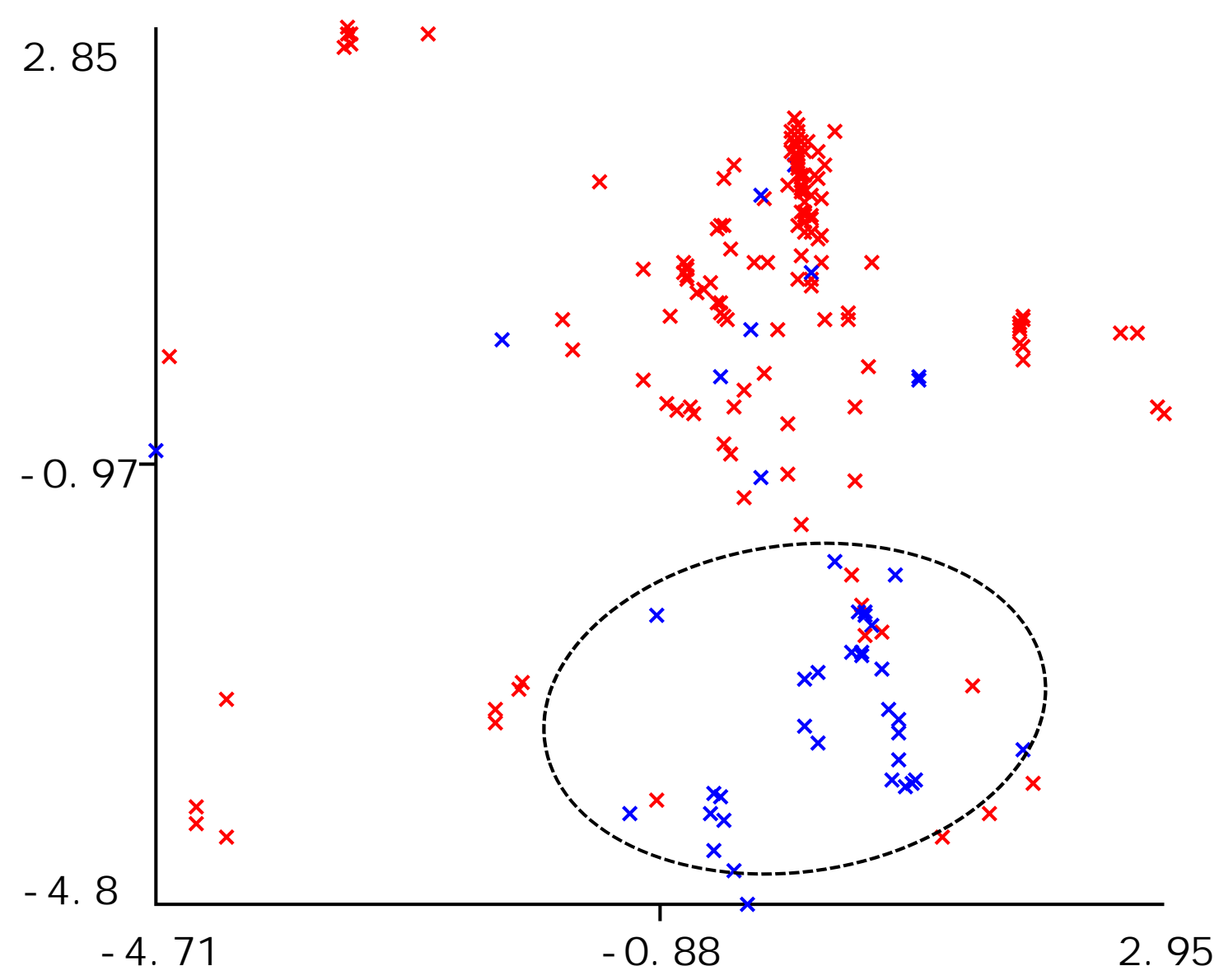

Figure 8.12: Selected projection to 2D of the $4 \mathrm{D}$ optimized PCA space. Blue crosses are Incorrect predictions. The ellipse shows a high concentration of Incorrect predictions that could explain the good classification of the model.

Use case: As discussed in Chapter 7, there is a growing number of large datasets that are constructed from textual corpora using automatic information extraction techniques such as Open Information Extraction (OpenIE) [72]. The output of such automatic techniques contains a set of high-quality triples along with a set of erroneous triples which should be considered as noise as illustrated in Figure 7.4. Thus, in order to have a high quality datasets, detecting noisy triples and removing them become essential steps.

Metrics: In this experiment, we wanted to identify noisy triples from automatically generated triples from textual data. Noisy triples are identified by three filters which classify if a given triple is Inconsistent, Generic, or Factually False. For each of these, 
we have used Precision and Recall as evaluation metrics. In this context, each filter works as a binary classifier such that each triple that passes through it will be either labelled positive or negative. For example, an inconsistent filer will mark a triple as positive if the algorithm finds it to be inconsistent and as negative otherwise. Based on that, we calculate the precision and recall using the following formulas.

$$
\begin{aligned}
\text { precision } & =\frac{\text { true positives }+ \text { true negatives }}{\text { all triples }} \\
\text { recall } & =\frac{\text { true positives }}{\text { expected positive triples }}
\end{aligned}
$$

Dataset: In this experiment, we have used the output of a commercial information extraction tool as our dataset which contained triples in the form of < subject, predicate, object $>$. The corpus used by the tools to generate the triples is Wikipedia. As our gold standard, we manually labelled a sample of 5,342 triples from the tool output. Each triple was labelled either Inconsistent, Generic, or Factual by human annotators. Generic and factual triples were further labelled with a truth value: True, False, or True in the past. Then we conducted three experiments to evaluate the algorithms using those manually labelled triples.

\section{Results:}

We evaluated the performance of the three filters proposed in the method for detecting different types of noise. The results are presented in Table 8.27.

Table 8.27: Experimental results

\begin{tabular}{|l|l|l|l|}
\hline Category & Configuration & Precision & Recall \\
\hline Inconsistent triple detection & & $86.84 \%$ & $62.26 \%$ \\
\hline \multirow{3}{*}{ Generic triple detection } & Standford NLP & $85.65 \%$ & $100 \%$ \\
\cline { 2 - 4 } & Open NLP & $78.16 \%$ & $100 \%$ \\
\cline { 2 - 4 } & Combined & $98.25 \%$ & $100 \%$ \\
\hline Factual false triple detection & & $85.12 \%$ & $91.26 \%$ \\
\hline
\end{tabular}

Discussion: As it can be seen in Table 8.27, all three filters achieve high precision and recall values for this experiment. As we have detailed in Section 7.2.5, the inconsistent triple detection algorithm used Linked Data profiling metrics to identify an abstract pattern for a triple using the type of subject and object and then assign it a score. As the results show, that information provides good signals for classifying inconsistent triples. Generic triple detection performed mainly based on NLP techniques such as part-of-speech tagging. Factual triple detection also uses Linked Data profiling 
metrics for aligning the triples extracted from the text with external knowledge graphs such as DBpedia.

\subsubsection{Repairing Hidden Links}

The third method proposed under $C_{4}$ is $\mathrm{C} 4.3$ a link repair technique based on $R D F$ Shapes. The hypothesis related to this method is that $\boldsymbol{H 8}$. The proposed link repair method based on RDF Shapes (C4.3) can achieve more than 80\% F1 in identifying and repairing broken links in Linked Data (i.e., entities that should be denoted by IRIs but are represented as strings).

Use case: As discussed in Chapter 7, Linked Data allows applications to traverse within, and even out of, a dataset thanks to use of global dereferenceable identifiers in the form of IRIs denoting entities. Nevertheless, we identified that it is a common problem that many entities that should be represented IRIs are being represented using literal strings. Because of this some entities are not linked diminishing the advantages of using Linked Data. Thus, in Chapter 7, we proposed a method for identifying strings that denote entities and replacing them with their corresponding entity IRIs using RDF Shapes and data profiling information.

We have formulated three sub-hypotheses related to this method.

- H8.1 Entities that should be represented as IRI but are presented as strings in data can be identified using data profiling information and RDF Shapes.

- H8.2 Such strings can be converted to their corresponding IRIs using named entity recognition techniques.

- H8.3 Repairing such links increases the connectivity of the RDF graph.

Metrics: For this, we perform three different experiments to evaluate the different aspects of the proposed method. The first evaluation is related to candidate identification, i.e., the detection of properties that relate two entities. Identification of such relations is important because it allows to detect strings that should be represented as IRIs. For this evaluation, we use precision and recall as the evaluation metrics. In this context, the precision refers to the fraction of correct candidates among the all candidates identified by the proposed method. Similarly, the recall refers to the fraction of the correct candidates identified by the method among all the correct instances in the input to the method.

The second evaluation is related the performance of the disambiguation of strings, i.e., once a string is identified as a candidate for converting to an IRI, how good is the method in finding the correct IRI for the given string. Similar to the previous 
evaluation, precision and recall are used as the evaluation metrics. In this context, the precision refers to the fraction of correctly disambiguated and linked strings among all disambiguated strings. When calculating the recall, we have calculated it using two different criteria, Recall-1 and Recall-2. In Recall-1, we simply calculate the fraction of correctly disambiguated and linked strings among all the strings were used as the input. Nevertheless, sometimes there is no entity with IRI in the dataset that the string can be linked to. In such cases, it is impossible for the method to link such strings to an IRI. Thus, to reflect a more realistic value, we define Recall-2 as the fraction of correctly disambiguated and linked strings among all the strings where a corresponding entity with IRI exists in the dataset.

The third evaluation is related to the improvement of connectivity of the RDF graph in the dataset. When string literals denoting entities are converted to their IRIs, some of the nodes in the RDF graphs get merged together and also new links are created between nodes. Thus, the connectivity of the RDF graph gets improved. We measure this improvement using the difference of a set of network metrics. First we measure, the difference in the number of edges in the RDF graphs before and after applying the method. When the number of edges increases, it means that there are more links between the nodes in the graph and the connectivity is increased. Secondly, we measure the number of components in the graph. In graph theory, a component is defined as a subgraph in which all nodes are connected but the given subgraph (the component) is not connected to any other subgraphs in the supergraph. When there are more components in the graph, it means there are more subgraphs that are not connected to each other. Thus, if the number of components are decreased, it indicates that the connectivity of the graph is increased. Thirdly, we measure the isolated nodes in the graph. Isolated nodes are a special case of components, i.e., the components of size one or nodes that are not connected to any other node in the graph. When the number of isolated nodes are decreased in the graph, it also provides an indication that the connectivity of the graph is increased. Finally, we consider the number of nodes in the largest component of the graph and the percentage of the largest component of the whole RDF graph. When the size of the largest component is increased, the connectivity of the graph increases.

Dataset: In this evaluation, we have used a subset of the Spanish DBpedia including the resources of types dbo:Athlete, dbo:SportsTeam, and dbo:SportsEvent.

\section{Experimental setup:}

We evaluate our work based on three dimensions. First, we evaluate our entity relation detection algorithm. The goal of this evaluation is to validate if the relations detected by the algorithm are correct (i.e., precision) and also the algorithms detects all entity relations (i.e., recall). We performed the evaluation by manually annotating all class-property combinations of the three selected classes: dbo:Athlete, 
dbo:SportsTeam, and dbo:SportsEvent. The annotation process was conducted by three reviewers who agreed on whether each property should be considered an entity relation or not.

\section{Results:}

Table 8.28 shows the evaluation results. It shows that the algorithm achieves high recall with a reasonable precision. We intentionally optimized the algorithm for recall because the false positives get filtered in the second step.

Table 8.28: Candidate identification performance

\begin{tabular}{|l|l|l|l|l|l|}
\hline \multirow{2}{*}{ Class } & Entity & \multicolumn{2}{|l|}{ Detected relations } & \multirow{2}{*}{ Prec. } & \multirow{2}{*}{ Recall } \\
\cline { 3 - 4 } & relations & Correct & Incorrect & & \\
\hline dbo:Athlete & 183 & 178 & 53 & $77.06 \%$ & $97.27 \%$ \\
\hline dbo:SportsTeam & 157 & 156 & 48 & $76.47 \%$ & $99.36 \%$ \\
\hline dbo:SportsEvent & 116 & 115 & 41 & $73.71 \%$ & $99.14 \%$ \\
\hline Total & 456 & 449 & 142 & $75.97 \%$ & $98.47 \%$ \\
\hline
\end{tabular}

Second, we evaluated the string to IRI conversion process using the candidate class-property combinations. Similar to the previous evaluation, this is performed by selecting a sample of 100 strings from each of the three classes selected before and manually annotating whether the linking is done correctly or not. Table 8.29 shows the evaluation results and in this step we have a high precision and low recall. The lower recall is due to the fact that some entities do not have a corresponding IRI in the KG. High precision in this step compensates the high recall and the medium precision in the previous step.

Table 8.29: String disambiguation performance

\begin{tabular}{|l|l|l|l|l|l|l|}
\hline \multirow{2}{*}{ Class } & \# of & \multicolumn{2}{|l|}{ Disambiguated } & \multirow{2}{*}{ Prec. } & \multirow{2}{*}{ Recall-1 } & \multirow{2}{*}{ Recall-2 } \\
\cline { 3 - 4 } & strings & $\#$ & Correct & & & \\
\hline dbo:Athlete & 100 & 51 & 50 & $98.04 \%$ & $50 \%$ & $87.72 \%$ \\
\hline dbo:SportsTeam & 100 & 44 & 44 & $100 \%$ & $44 \%$ & $91.67 \%$ \\
\hline dbo:SportsEvent & 100 & 58 & 55 & $94.83 \%$ & $56 \%$ & $85.94 \%$ \\
\hline Total & 300 & 153 & 149 & $97.38 \%$ & $49.67 \%$ & $88.76 \%$ \\
\hline
\end{tabular}

Third, we selected a subset of Spanish DBpedia containing the data about instances of dbo:Athlete, dbo:SportsTeam, dbo:SportsEvent, and dbo:PopulatedPlace (i.e., original graph). Then, we ran our algorithms for detecting entity strings and converting them to entity IRIs to generate a new graph (i.e., repaired graph). Then, we calculated the number of graph components to check the increase of connectivity in the RDF graph. 
Table 8.30: Improvements in connectivity

\begin{tabular}{|l|r|r|r|r|r|}
\hline \multirow{2}{*}{ Graph } & \multirow{2}{*}{ edges } & \multirow{2}{*}{ comeponents } & \multirow{2}{*}{ isolated } & \multicolumn{2}{|c|}{ largest component } \\
\cline { 5 - 6 } & & & & size & \% of total \\
\hline Original & 828,310 & 119,623 & 112,331 & 168,128 & $54.28 \%$ \\
\hline Repaired & $1,035,912$ & 99,137 & 93,507 & 192,805 & $64.16 \%$ \\
\hline Connectivity & $+207,602$ & $-20,486$ & $-18,824$ & $+24,677$ & \\
difference $\Delta$ & $(+25.06) \%$ & $(-17.12 \%)$ & $(-16.76 \%)$ & $(+14.68 \%)$ & $(+9.88 \%)$ \\
\hline
\end{tabular}

Table 8.30 shows that, as a result of increasing the number of edges, the connectivity increases significantly according to several metrics.

When there is a reduction in the number of components in a graph, the graph increases its connectivity. In the repaired graph, we reduce the number of components by $17.12 \%$ due to the additional links. Another measure of connectivity is the number of components that have only one node, that is, isolated nodes. These nodes are not connected to any other nodes in the graph. When connectivity increases, the number of isolated nodes decreases. In the repaired graph, we get a reduction of $16,76 \%$ in the number of isolated nodes.

Also the number (or the percentage) of nodes in the largest component provides a good indicator of the connectivity. When this number increases, more nodes in the graph are in the largest subgraph, as a result of connecting with most of the other nodes. In our experiment we have increased the largest component by $14.68 \%$. Figure 8.13 shows the size of the components, ordered by size (number of nodes in the component), for the original graph. As we discussed before, we were able to move a large number of nodes $(24,677)$ from the long tail into the main largest component and most of them were previously isolated components $(18,824)$.

\section{Discussion:}

It is worth to note that several issues were identified during the evaluation, as part of the manual annotation, which makes the conversion process not always straightforward. Some properties were ambiguous; for instance, the property "inicio" (meaning start) in the Athlete instances is being used to relate to either (a) the club where an athlete began her career or (b) the year she or he begun. In such cases, it is hard to detect whether it is an entity relation or not, because it acts as both depending on which sense it is used. In other cases we have detected that some string objects actually denote entity lists rather than a single entity. For example, "equipos" (teams) has string values such as "*Los Angeles Lakers *Chicago Bulls *Toronto Raptors *New Orleans". Such values would need to be preprocessed to extract each individual entity. We also noticed that often URLs are encoded as strings whereas they could be represented as IRIs. To handle these cases, we propose to perform a 


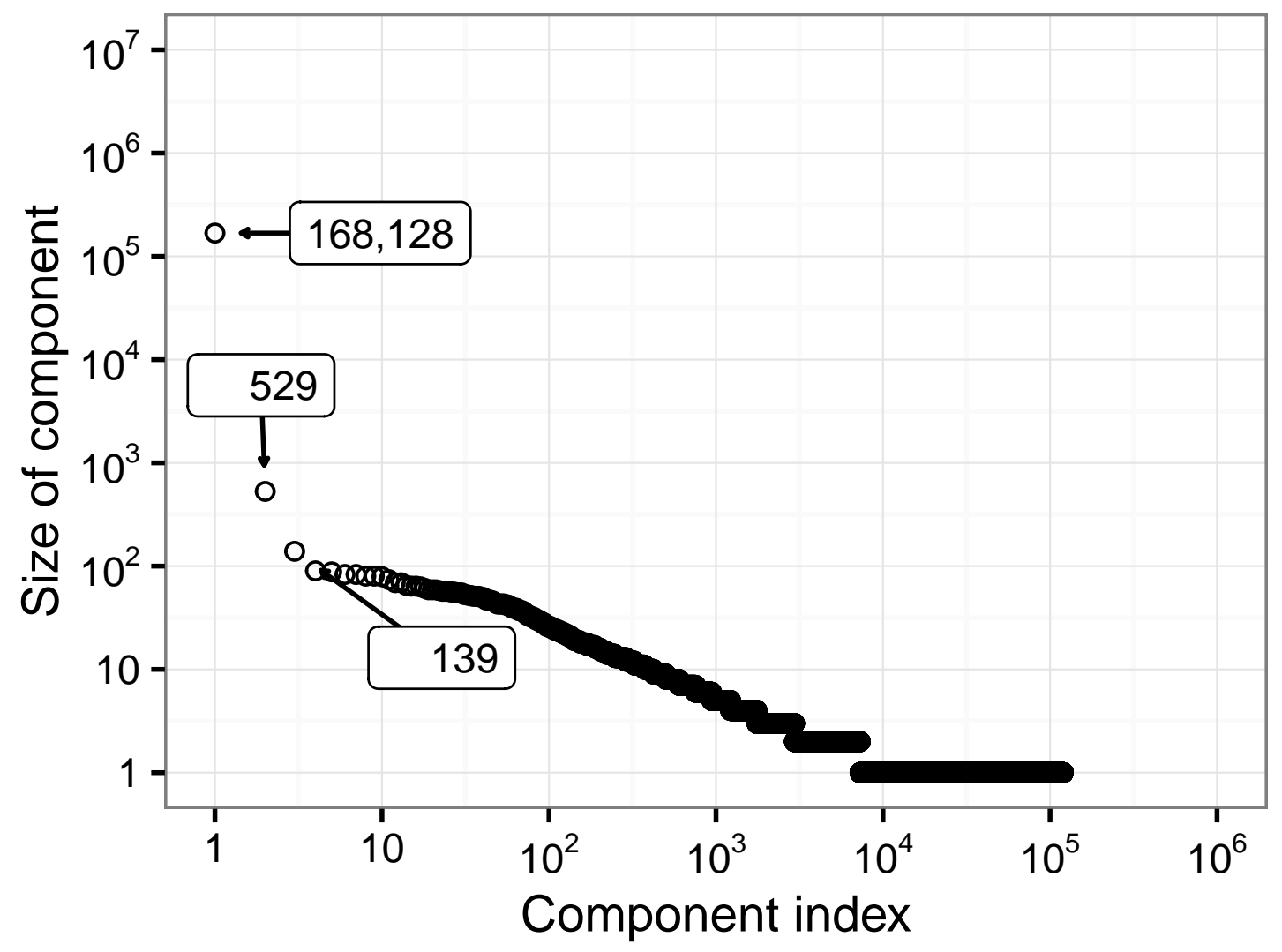

Figure 8.13: Ranking of component sizes in the original graph. Components with more than 100 nodes are labelled with the number of nodes.

quick regex check to detect if the string is a URL and convert it to an IRI directly.

Finally, we have to consider that it is not always possible to convert a string to an IRI, even when we correctly detect that a given string denotes an entity. The corresponding resource may not exist in the KG and thus we might not have an identifier to link to. For instance, in the case of DBpedia, even if a resource exists in the real world, it might not have an entry in DBpedia if there is not a Wikipedia page for it. This is out of the scope of this work.

\subsubsection{Type Prediction Method using Binary Classifiers}

The fourth method purposed under $\mathrm{C} 4$ methods for automatic quality assessment and repair using Linked Data profiling metrics and RDF Shapes is $C_{4.4}$ a type completion method using Linked Data profiling metrics. The hypothesis related to this method is that $\boldsymbol{H 9}$. the proposed type completion method (C4.4) using Linked Data profiling 
metrics can achieve an accuracy of $80 \%$ when predicting types for instances.

Use case: Type information plays an important role in making the data on the Semantic Web easily queryable and reusable. Nevertheless, in some datasets there are a significant amount of resources without type information and does not contain enough information to infer their types only using reasoning. Thus, this thesis proposes a data-driven approach for predicting type information of instances utilising machine learning techniques data profiling metrics as input features.

Metrics: The goal of this evaluation is to measure the performance of the type prediction method proposed in this section. The proposed method builds a type predictor model for each class as a binary classifier which predicts if a given resource in the dataset belongs to the given class or not. In this context, as illustrated in Table 8.31, we can identify four types of predictions: true positives, false positives, true negatives, and false negatives.

Table 8.31: Possible predictions of type prediction model for class X

\begin{tabular}{|l|l|l|l|}
\cline { 3 - 3 } \multicolumn{2}{c|}{} & \multicolumn{2}{c|}{ A resource of } \\
\cline { 3 - 4 } \multicolumn{2}{c|}{} & \multicolumn{1}{c|}{ Class X } & Other \\
\hline \multirow{2}{*}{ Predicted as } & Class X & True Positives & False Positives \\
\cline { 2 - 4 } & Other & False Negative & True Negatives \\
\hline
\end{tabular}

In order to measure the performance of the type predictors, we have used the classification accuracy.

$$
\text { accuracy }=\frac{\text { true positives }+ \text { true negatives }}{\text { true positives }+ \text { false positives }+ \text { true negatives }+ \text { false negatives }}
$$

Dataset: In this evaluation, we have used the English and Spanish DBpedia datasets. We have selected a sample from each dataset as illustrated in Table 8.32. For the selected sample data, we have removed the type information from each resource and used the prediction models to predict the type only using the Linked Data profiling metrics related to the presence of different properties. We have used the type information in those two datasets as the gold standard for testing the performance of the proposed method and baseline.

\section{Experimental setup:}

The main hypothesis evaluated in this experiment is that the type prediction method utilising binary classifiers with Linked Data profiling metrics as input has 
Table 8.32: Datasets used in the experiments

\begin{tabular}{|c|c|c|c|c|c|c|c|c|}
\hline Source & ID & Feat. & \multicolumn{2}{|c|}{ Labelled Instances } & ID & Feat. & \multicolumn{2}{|c|}{ Labelled Instances } \\
\hline \multirow{4}{*}{$\begin{array}{l}\text { Spanish } \\
\text { DBpedia }\end{array}$} & \multirow{2}{*}{ ES-1 } & \multirow{2}{*}{132} & Positive & Holiday $(809)$ & \multirow{2}{*}{ ES-2 } & \multirow{2}{*}{144} & Postive & Holiday $(809)$ \\
\hline & & & Negative & Person(1000) & & & Negative & $\begin{array}{l}\text { Person(500), } \\
\text { Event }(500)\end{array}$ \\
\hline & \multirow{2}{*}{ ES-3 } & \multirow{2}{*}{299} & Postive & \multicolumn{5}{|l|}{$\operatorname{Holiday}(809)$} \\
\hline & & & Negative & \multicolumn{5}{|c|}{$\begin{array}{l}\text { Activity, Agent, AnatomicalStructure, Award, } \\
\text { Biomolecule, ChemicalSubstance, Colour, } \\
\text { Currency, Device, Disease, Event, Food, } \\
\text { Language, MeanOfTransportation, Species, } \\
\text { SportCompetitionResult, TopicalConcept, } \\
\text { Work - } 50 \text { each, Place(100) }\end{array}$} \\
\hline \multirow{4}{*}{$\begin{array}{l}\text { English } \\
\text { DBpedia }\end{array}$} & \multirow{2}{*}{ EN-1 } & \multirow{2}{*}{132} & Positive & Holiday(1175) & \multirow{2}{*}{ EN-2 } & \multirow{2}{*}{144} & Positive & Holiday(1175) \\
\hline & & & Negative & Person(1175) & & & Negative & $\begin{array}{l}\text { Person(588), } \\
\text { Event }(587)\end{array}$ \\
\hline & \multirow{2}{*}{ EN-3 } & \multirow{2}{*}{299} & Positive & \multicolumn{5}{|l|}{$\operatorname{Holiday}(1175)$} \\
\hline & & & Negative & \multicolumn{5}{|c|}{$\begin{array}{l}\text { Activity, Agent, AnatomicalStructure, Award, } \\
\text { Biomolecule, ChemicalSubstance, Colour, } \\
\text { Currency, Device, Disease, Event, Food, } \\
\text { Language, MeanOfTransportation, Place, } \\
\text { Species, SportCompetitionResult, Topical- } \\
\text { Concept - } 65 \text { each, and Work(70) }\end{array}$} \\
\hline
\end{tabular}

better performance than the baseline. As the baseline for type prediction, we have used a keyword based type prediction approach where we have used the presence of a given keyword in the resource label (rdfs:label) and resource description (dbo:abstract) to predict the type. We have designed three distinct experiments to evaluate the aforementioned hypothesis under the following conditions:

- A Machine Learning binary classifier can be used for type prediction with the presence of properties as features with high precision.

- The accuracy of the aforementioned classifier will depend on the diversity of data (i.e., the number of different types of instances in data)

- A model learned from one dataset can be used to predict types of another dataset. (e.g., learning from Spanish DBpedia and predicting on English DBpedia or vice versa).

Experiment 1: The objective of the first experiment is to evaluate H4.5.1, that a machine learning classifier can be used to predict the type of a resource using as features the presence of properties. In this experiment, we build a supervised type predictor for Holiday class using as features a set of 132 properties of the DBpedia 
ontology (i.e., all the properties found in training data). All the features are binary $(0 / 1)$ nominal attributes, that is, a given resource has (or has not) a given property, not considering the value or the property.

For this experiment we used the dataset ES-1 extracted from the Spanish DBpedia (Table 8.32), which contains 809 instances of Holiday (labelled as positive) and 1000 instances of Person (labelled as negative). We selected the class Person because it is conceptually different from Holiday, as a first test to distinguish between instances of Holiday and Person.

Using the dataset ES-1, we ran a 10-fold stratified cross validation and the results are presented in Table 8.33. The proposed approach achieved an accuracy of $\mathbf{9 9 . 0 6 \%}$ for method J48 [195] (only 17 instances were incorrectly classified in 1,809), and $\mathbf{9 9 . 2 8 \%}$ for Random Forest [42]. We can visualise the relation between instances computing the distance between instances as its euclidean distance in the vector space defined by its features. In this way we can compute a distance matrix and create a graph. Applying a force-directed layout such as Fruchterman-Reingold algorithm [85] we get the graph show on the right side of figure 8.14. In that figure we can see a clear separation between Holiday instances and Person instances, with only a few instances of Person in the cluster of Holiday, in concordance with the high accuracy of the prediction.

Table 8.33: Results of experiment I

\begin{tabular}{|l|l|l|}
\hline \multirow{2}{*}{ Approach } & \multicolumn{2}{|c|}{ Dataset } \\
\cline { 2 - 3 } & ES-1 & EN-1 \\
\hline Type prediction with Random Forest & $\mathbf{9 9 . 2 8 \%}$ & $\mathbf{9 8 . 6 4 \%}$ \\
\hline Type prediction with J48 & $99.06 \%$ & $98.38 \%$ \\
\hline Baseline & $54.34 \%$ & $62.26 \%$ \\
\hline
\end{tabular}

Experiment 2: The objective of the second experiment is to evaluate the H4.5.2, that the accuracy of the aforementioned classifier depends on the diversity of the knowledge graph. For this experiment, in addition to dataset ES-1, two other datasets were used. In all these three datasets, the positive training data remained the same, i.e., in each of the three datasets there are 809 instances of Holiday. However, the negative training dataset ES-2 includes 500 Person instances and 500 Event instances. By including instances of Event, which are conceptually closer to Holiday, we increase the complexity of the training/test data.

The 10-fold stratified cross validation achieves an accuracy of $\mathbf{9 1 . 4 9 \%}$ for method J48, and 91.21\% for Random Forest. Notice the high values despite Event is the closest class to Holiday from the 162 classes (considering all levels not just first level) with instances in the Spanish DBpedia. 

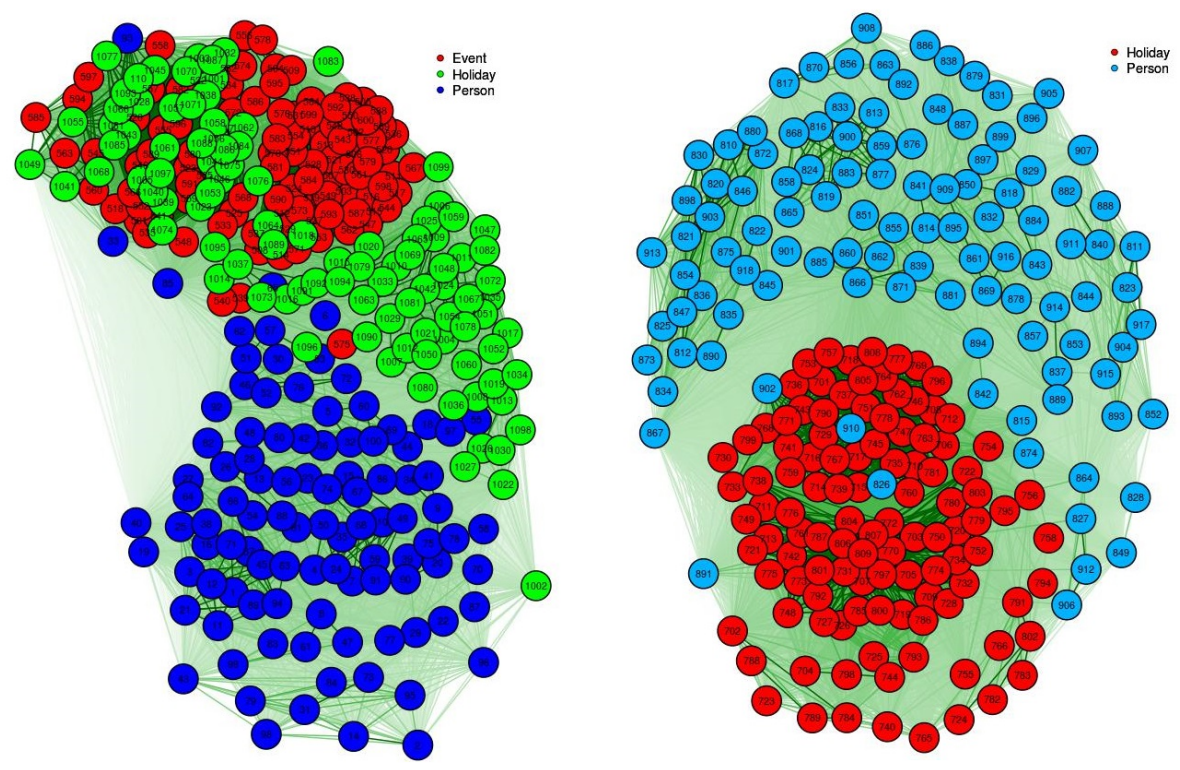

Figure 8.14: Identifying Holidays in a knowledge graph. Right) in a mix of Holidays and Persons, and Left) in a mix of Holidays, Persons and Events. Notice that the colors for instances of Holiday and Person differ in each graph.

The knowledge graph, using the previously explained method, is the left graph in figure 8.14. In this graph we see a clear separation between instances of Holiday and Person, but there is a mix between Holiday and Event.

In dataset ES-3, the negative training data includes instances of all ten 1st level classes (i.e., direct subclasses of owl:Thing) from the Spanish DBpedia. These classes are listed in table 8.32. The 10-fold stratified cross validation achieves an accuracy of $\mathbf{9 4 . 9 7 \%}$ for method J48, and $\mathbf{9 5 . 6 3 \%}$ for Random Forest. We get higher values than in ES-2 because there is a proportional lower number of classes conceptually similar to Holiday (like Event).

Therefore we conclude that this method predicts with high accuracy when the diversity of classes is high and, even in the worst case, when there are few classes but very similar to the trained class, the achieved accuracy is high.

Table 8.34: Results of experiment II

\begin{tabular}{|l|l|l|l|l|}
\hline \multirow{2}{*}{ Approach } & \multicolumn{4}{|c|}{ Dataset } \\
\cline { 2 - 5 } & ES-2 & EN-2 & ES-3 & EN-3 \\
\hline Type prediction with Random Forest & $91.21 \%$ & $\mathbf{9 3 . 6 0 \%}$ & $\mathbf{9 5 . 6 3 \%}$ & $\mathbf{9 2 . 7 7 \%}$ \\
\hline Type prediction with J48 & $\mathbf{9 1 . 4 9 \%}$ & $92.60 \%$ & $94.97 \%$ & $91.70 \%$ \\
\hline Baseline & $54.01 \%$ & $61.91 \%$ & $53.51 \%$ & $59.49 \%$ \\
\hline
\end{tabular}


Experiment 3: The objective of this third experiment is to evaluate H4.5.3, that a model learned from one dataset can be used to predict types of another dataset. In this case, we first trained the model by using data from the Spanish DBpedia (ES-1) and testing the model by using the data from the English DBpedia (EN-1). For this case, we achieved an accuracy of $98.63 \%$. Then, we repeated the experiment with a more diverse setting using datasets ES-3 and EN-3. Again, we trained the model by using data from the Spanish DBpedia (in this case, ES-3) and testing the model by using the data from the English DBpedia (EN-3). For this setting, we achieved an accuracy of $75.52 \%$. Finally, we trained the model ES-2 and tested it using EN-2. This final setting does not yield good results (i.e., just $5 \%$ over the $50 \%$ random guessing baseline) and that is mainly because of the conceptual similarity between Holiday and Event. We plan to further investigate how to improve such cases in the future. A summary of all experimental results are presented in Table 8.35.

Table 8.35: Results of experiment III

\begin{tabular}{|l|l|l|l|l|l|l|l|}
\hline \multirow{3}{*}{ Approach } & \multicolumn{7}{|c|}{ Dataset } \\
\cline { 2 - 8 } & Training & ES-1 & EN-1 & ES-2 & EN-2 & ES-3 & EN-3 \\
\cline { 2 - 8 } & Testing & EN-1 & ES-1 & EN-2 & ES-2 & EN-3 & ES-3 \\
\hline Random Forest & $98.63 \%$ & $91.40 \%$ & $55.72 \%$ & $90.30 \%$ & $75.52 \%$ & $94.20 \%$ \\
\hline Baseline & $54.34 \%$ & $62.26 \%$ & $50.01 \%$ & $61.91 \%$ & $53.51 \%$ & $59.49 \%$ \\
\hline
\end{tabular}

\section{Discussion:}

In Linked Data represented using Semantic Web techniques type information is quite useful for many applications such as efficient querying or visualisation of data. Thus, completing type information in datasets improves the quality and usefullness of the dataset.

In this proposed method, our intuition is that in general the relations or the attributes provide signals about what the type of the instance is. Thus, we can use the Linked Data profiling metrics from a large number of instances to train a classifier to predict types based on the presence of relations in an instance. The classifier learns which combinations of relations are associated with a given type.

Using the three experiments presented in this section, we demonstrate that Linked Data profiling metrics can be effectively used as features for a classifier that is capable for predicting types of instances. In all three experiments, the proposed classifier had much better performance than the baseline approach. 


\section{Chapter 9}

\section{Conclusions and Future Work}

With the growth of data-intensive and knowledge-driven applications in the current era of digital transformation, there is an essential need for large amounts of data for them to use. If such data is in a structured and machine-processable format with explicit semantics, their value increases because such data is much easier to exploit in knowledge-driven applications. Thus, Linked Data represented using Semantic Web standards such as RDF and OWL can bring a lot of benefits. Nevertheless, as discussed throughout this thesis, one of the essential requirements for such data is to have a high quality.

Until recent times, Linked Data generation processes have focused more on quantity than quality. This led to datasets with billions of triples; however, for these datasets to be used in practical applications, there was a need for scalable quality assessment and repair methods. To this end, the main objective of this thesis was to propose novel and scalable methods for automatic quality assessment and repair of Linked Data by addressing the open research problems identified in Chapter 3. We have identified several research problems from both a methodological perspective and a technological perspective and established four main objectives. The objectives of the thesis are fulfilled by the main contributions of the thesis.

In this chapter, we conclude by reviewing the main contributions of the thesis. Furthermore, we describe the practical exploitation of the results of the thesis. Finally, we provide some insights on future research directions that can be thought of as a continuation of the work conducted in this thesis.

\subsection{Review of Main Contributions}

For quality assessment and repair methods, it is important that the quality of a dataset and relative improvements to it by repair methods can be measured and represented in a standard manner. With this respect, we have identified the following 
open issues while analysing the state of the art on Linked Data quality. There is a high diversity in terms that refers to quality aspects in Linked Data, which leads to difficulties in comparing and benchmarking evaluation results, as well as in selecting the right data source according to a set of particular quality needs. More concretely, there is a lack of a quality model for Linked Data that specifies a set of quality characteristics and metrics related to the quality characteristics in the domain of Linked Data with a unique terminology as a reference for data quality specification and evaluation. Furthermore, we noted that the existing quality characteristics and metrics for Linked Data found in the literature were not aligned with the models proposed by standardisation initiatives such as ISO.

- To this end, the first objective of the thesis was to provide means for representing quality assessment results along with all associated metadata in a comparable manner using well-established standards such as ISO 25012 for data quality and the W3C Data Quality Vocabulary. This allows the quality assessment results about datasets to be published in a standard and interoperable manner.

To achieve the first objective, we have created $L D Q M$, a quality model for Linked Data, which provides a unique terminology and reference for Linked Data quality specification and evaluation. The proposed quality model specifies a set of quality characteristics and quality measures related to Linked Data, together with formulas for the calculation of those measures. It contains 15 quality characteristics, 89 base measures, 23 derived measures, and 124 quality indicators. LDQM also provides an ontology extension of the W3C Data Quality Vocabulary that can be used to capture Linked Data quality information and provide an RDF representation. LDQM has been evaluated to test the hypothesis that it satisfies a set of characteristics that are relevant for a quality model, namely, comprehensiveness, applicability, understandability, and consistency. The evaluation shows that the quality model stratifies all four desired characteristics. LD Sniffer, a tool for evaluating Linked Data accessibility, was built to evaluate the hypothesis that LDQM can help the development of Linked Data quality assessment tools. The evaluation shows that the quality model is useful in the process of developing quality assessment tools.

Regarding Linked Data profiling, we have identified the following gaps in existing research. Even though data profiling metrics can provide valuable inputs to quality assessment methods and they have been widely used in other paradigms such as relational data, the current Linked Data profiling methods do not extract information with the level of detail required for quality assessment and repair use cases. This hinders the usage of data profiling metrics in data quality assessment and repair methods. Furthermore, there is a lack of Linked Data profiling tools that can produce 
comprehensive profiling metrics with the level of detail required by data quality use cases.

- To this end, the second objective of the thesis was to identify information that could be used in quality assessment and repair methods and could be generated automatically generated by profiling large-scale datasets. More concretely, our aim was to improve RDF data profiling techniques focusing on the Linked Data quality assessment and repair use cases. This was done by extending metrics collected by the current RDF data profiling tools to include fine-grained profiling information that is needed for quality assessment and by proposing an RDF dataset description model for representing data profiling information, dataset statistics, and other data pattern information with a sufficient level of detail.

Loupe, a framework for Linked Data profiling, was developed to achieve this objective. The Loupe Linked Data profiling framework consists of (a) an extended dataset description model with fine-grained metrics, (b) a scalable Linked Data profiling toolkit, (c) a web portal to visually explore the profiling metrics, and d) a REST API for ease of integrating the toolkit with other systems. The Loupe extended dataset description model defines 84 Linked Data profiling metrics that are useful for quality assessment and repair tasks. We have evaluated eight state-of-the-art dataset description models and demonstrated that the current dataset description models are not expressive enough to represent the fine-grained Linked Data profiling metrics needed for quality assessment and repair tasks. Furthermore, we showed that the Loupe dataset description model is more expressive than the current dataset description models. We have used the Loupe Linked Data profiling toolkit to evaluate 26 thousand datasets containing 34 billions of triples. Through an experiment, we demonstrated that the execution time of the Loupe Linked Data profiling framework scales linearly with respect to the dataset size and richness of vocabularies used and that it can process large datasets in a reasonable time. The Loupe Web portal has been visited more than 40,000 times by $3000+$ unique visitors from 87 countries. These statistics provide evidence that it is a useful tool for the Semantic Web community. Furthermore, a method that used the Linked Data profiling framework won the ISWC Semantic Web Challenge $201 \%$.

For validating data quality requirements and performing the necessary actions for repairing data, a set of validation constraints or rules are necessary. Such validation constraints define the characteristics or quality standards that the data has to satisfy. In the state of the art methods, these validation constraints are mostly manually formulated by domain experts, which is a tedious process that requires a significant amount of manual labour. Furthermore, it requires human experts to have good knowledge about the domain. Such approaches do not scale for large datasets. There 
is a lack of methods for inducing integrity constraints automatically from data and generating validation inputs, such as RDF Shapes.

- To this end, the third objective was to propose methods for automatically deriving Linked Data validation constraints for large datasets. More concretely, to develop an automatic RDF Shape Induction technique to generate constraints using a data-driven approach without requiring manual effort and to combine the aforementioned constraints into RDF Shapes.

To achieve this objective, we have developed a data-driven approach for inducing integrity constraints for RDF data using Linked Data profiling metrics. The constraints generated can be combined into RDF Shapes and they can be used to validate $\mathrm{RDF}$ graphs. The proposed method is based on machine learning techniques to automatically generate RDF shapes using profiling metrics as features. The method consists of five phases including (a) task formulation, (b) feature engineering, (c) gold standard preparation, (d) algorithm selection, and (e) constraints and RDF Shape generation. In the experiments, the proposed approach achieved $98.81 \%$ F1 in deriving maximum cardinality constraints, $97.30 \% \mathrm{~F} 1$ in deriving minimum cardinality constraints, and $95.94 \%$ in deriving range constraints for a subset of the English DBpedia data using a Random Forest model. In each of these cases, the proposed method showed approximately $20 \%$ improvement compared to the baseline validating the hypothesis that the proposed automatic RDF Shape Induction method based on machine learning algorithms using Linked Data profiling metrics as features, has a higher accuracy than the baseline approach.

Similar to the case of constraint generation, the current Linked Data quality assessment techniques generally require a large amount of manual effort, which makes such approaches not feasible for large-scale Linked Data datasets with millions of triples. Furthermore, most of the research on Linked Data quality is primarily focused on the quality assessment aspect, and not on repair. This is natural as Linked Data quality research is still in an early stage and quality assessment is the first step towards ensuring data quality. However, repairing data is also an essential aspect to improve the quality of data. With this respect, there is a lack of methods for repairing the data quality issues in Linked Data.

- To this end, the fourth objective was to develop methods for automatic quality assessment and repair using Linked Data profiling metrics and RDF Shapes. Such methods and techniques can reduce the manual effort required for quality assessment and repair and will enable efficient quality assessment and repair of large-scale Linked Data datasets such as DBpedia or Wikidata.

To achieve the fourth objective, we have developed four automatic quality assessment and repair methods. 
The goal of the first method is to detect inconsistent mappings that are used to generate Linked Data. This method allows detecting quality issues early in the Linked Data generation process. The proposed method uses a machine learningbased approach to build a predictive model which can detect incorrect mappings by analysing the characteristics of the data generated by them. Linked Data profiling metrics were used as features for training the predictive model. In the evaluation, we showed that the proposed method achieves $93 \%$ accuracy in identifying inconsistent mappings. These results helped to detect incorrect mappings in DBpedia and to correct them. Furthermore, this approach inspired the idea for a Google Summer of Code project ${ }^{1}$ in 2018, which the author co-mentored.

The goal of the second method was to assess and repair triples extracted from text using Open Information Extraction tools. The noisy triples were identified using three filters that detected Inconsistent, Overly Generic, and Factually False triples. The filters were designed as binary classifiers that detected if a triple falls into a given category using features generated by Linked Data profiling metrics and part-of-speech tagging. In the experiments, we demonstrated that the inconsistent triple detection filter, generic triple detection filter, and factual false triple detection filter achieved precisions of $86.84 \%, 98.25 \%$, and $85.12 \%$ respectively.

The goal of the third method is to repair links in data. One of the key advantages of using Linked Data is that data consumers can traverse through the typed links of the graph to discover related entities denoted by global and dereferenceable identifiers (i.e., IRIs). This characteristic is in the very core of Linked Data, as stated in the first and the fourth principles: "Use URIs as names for things" and "Include links to other URIs, so that they can discover more things". Nevertheless, sometimes strings are used instead of URIs. To remedy this, we propose an approach for identifying such strings and replacing them with their corresponding entity IRIs. The proposed approach is based on identifying relations between entities based on both ontological axioms as well as Linked Data profiling metrics and converting strings to entity IRIs based on the types of entities linked by each relation. Our approach showed $98 \%$ recall and $76 \%$ precision in identifying such strings and $97 \%$ precision in converting them to their corresponding IRIs. Furthermore, we analysed how the connectivity of the KG is increased when new links are added to the graph as a result of this method. The experiments show that this link repair technique could add 25\% more new links and improve the overall connectivity by $17 \%$.

Finally, the goal of the fourth method is to improve the completeness of Linked Data by adding missing type information to instances. The type information about resources in Linked Data plays a vital role in making them queryable and reusable. Nevertheless, in some datasets there are a significant amount of resources without type information (e.g., 1.1 million entities in DBpedia). This could be due to various

\footnotetext{
${ }^{1}$ https://github.com/dbpedia/GSoC/issues/15
} 
reasons such as deficiencies in the data generation or transformation process (e.g., lack of mappings) or deficiencies in source data (e.g., missing type information). The fact that data is incomplete and could contain noise makes it difficult to use classic type inference by reasoning to complete the missing type information [187]. We have developed a repair technique to predict missing type information using binary classifiers that use Linked Data profiling metrics about the attributes or the relations of a given instance as features to predict if the instance belongs to a particular class. We showed that an accuracy of $99.28 \%$ could be achieved with the proposed method when the dataset has only two classes and of $95.63 \%$ when the dataset has 20 classes.

\section{$9.2 \quad$ Future Work}

This thesis presents several advances to the research topic of Linked Data quality assessment and repair; nevertheless, there are still some open problems that have not been addressed in this thesis, and new research directions have come out as a consequence of the advances proposed in it. During this thesis, we have identified several future directions that would be interesting as follow up research:

- In this thesis, we have mainly focused on one of the main quality dimensions of Linked Data, that is, consistency. RDF Shape induction and Linked Data profiling metrics were used to develop methods that could perform quality assessment and repair related to inconsistencies in data. In addition, some other dimensions such as accessibility, accuracy, and completeness were also partially covered in some of the quality assessment and repair methods. Nevertheless, there are many other quality dimensions such as credibility, compliance, correctness, traceability, or understandability. The logical next step would be to explore how Linked Data profiling or other automatic techniques can be used to build scalable methods to assess and repair quality issues related to other dimensions that were out of the scope of this thesis.

- Continuing on the same line of thought of the previous point, one concrete research direction would be to use Linked Data profiling metrics along with pretrained language models to assess and repair quality issues related to correctness and completeness. Many datasets in the Linked Data cloud have a corresponding natural language text associated with them; for instance, Wikidata / DBpedia entities are associated with a Wikipedia article about the entity. If there are no directly associated documents, web searches or other information retrieval methods could be used to collect text related to a given triple [215]. Because language representation models such as BERT [60], XLNet [234], RoBERTa [150] have shown significant improvements in natural language processing tasks in 
recent years, it would a promising research direction to combine Linked Data profiling metrics from data and pre-trained language representations from their corresponding natural language text to develop quality assessment and repair techniques for quality dimensions that require additional background knowledge, such as correctness or completeness.

- In this thesis, we have used classical machine learning algorithms for classification and regression such as Random Forrest, SVM, or Naive Bayes. In recent years, Deep Learning [146] based on artificial neural networks have significantly improved the state-of-the-art in many domains, such as natural language processing, speech recognition, machine translation, object recognition and classification. Different deep learning architectures such as convolutional neural networks, recurrent neural networks, and deep belief networks have been used to address specific problems and are known to excel in the aforementioned domains. Thus, another potential research direction is to apply deep learning algorithms for automatic RDF Shape induction and Linked Data quality assessment and repair methods. One key challenge for deep learning applications is to find a large amount of labelled data for training. Linked Data profiling metrics could be used to generate labelled training data for such deep learning applications in a distantly supervised manner [172].

- Linked Data can be essentially thought as graphs of entities as nodes and relations as edges. Knowledge graph embedding is a way to learn a representation of the graph in continuous vector spaces, in which, both entities and relations can be represented as vectors [228]. Such embeddings allow performing operations on the graph more efficiently. For instance, the knowledge graph embeddings can be used for tasks such as link prediction, triple validation, or relation fact extraction [230]. RDF2Vec, one popular graph embedding model for Linked Data, converts the graph into a set of sequences of entities and uses those sequences to train a neural model in which each entity is represented as a vector. One research direction would be to explore the feasibility of using Linked Data profiling metrics for training graph embeddings.

- Recently Neuro-Symbolic AI $[13,154]$ approaches are getting much attention in the research community. The main idea behind Neuro-Symbolic approaches is to combine (a) symbolic knowledge represented using conventional logical knowledge representation languages that can be easily understood and explained by humans with (b) sub-symbolic knowledge represented as embeddings or vectors of numbers and learned through neural networks based representation learning. Thus, one promising research direction would be to explore NeuroSymbolic approaches for quality assessment and repair. Profiling the symbolic 
knowledge represented as Linked Data could provide valuable features for training the neural models and the combination of both symbolic and subsymbolic knowledge could be used in novel quality assessment and repair methods.

\subsection{Exploitation}

Finally, the contributions of this thesis have been already exploited by several research projects. In this section, we provide details of such exploitations.

\subsubsection{Linked Data Profiling Framework}

Chapter 6 discusses how data profiling information is used for RDF Shape Induction and Chapter 7 demonstrates how it is used in methods and techniques for quality assessment and repair in various use cases. In addition to those, the Loupe Linked Data profiling framework was used for performing various other tasks as discussed below.

3Cixty 3Cixty is a semantic web platform that enables to build real-world and comprehensive knowledge bases in the domain of culture and tourism for cities. The 3Cixty platform has been first used in the Expo Milano 2015 [205], where a specific knowledge base for the city of Milan has been developed, and it has been extended further with the development of knowledge bases for the cities of Nice and London. The 3Cixty knowledge bases contain descriptions of events, places (sights and businesses), transportation facilities and social activities, collected from numerous static, near- and real-time local and global data providers, including Expo Milano 2015 official services in the case of Milan, and numerous social media platforms. When deploying 3cixty on a regular basis, the developers have observed the imperial need of following an agile process. 3Cixty knowledge base is continuously evolving, since new cultural events are proposed every day, while some other resources cease to exist, e.g., a business has moved or has closed or is changing its opening hours. Thus, 3Cixty needed an approach to validate the evolution of the content of a knowledge base while maintaining a high level of quality. Furthermore, it was needed to monitor the growth of the knowledge base providing a high-level view of its evolution.

To this end, 3Cixty used a methodology suitable for quality assessment of continuously evolving knowledge bases that are consumed by real-world applications. The main objective of the methodology is to ensure that the periodically generated datasets (e.g., on a daily basis) meet the quality requirements of the use cases. The methodology consists of two main phases: (i) exploratory testing of the KB using dataset statistics and common patterns, and (ii) fine-grained analysis of the KB 
aligned with the quality requirements. For exploratory testing, the 3Cixty team used the Loupe Linked Data profiling framework [168].

Exploratory testing (also known as ad-hoc testing) is a branch of blackbox software testing where the testers simultaneously learn, design and execute tests in an exploratory manner [118]. In exploratory software testing, a tester would explore the software capabilities without a pre-defined test plan and design the tests on the fly based on the information gained while testing. This same idea, which is quite successful in the software industry [119], can be adapted to testing knowledge bases. In knowledge bases, exploratory testing can be performed by a tester by executing different queries on the knowledge base, analyzing them, and incrementally planning more queries based on the knowledge acquired. This requires the testers to write a lot of boiler-plate queries for extracting information about different aspects such as the usage of classes or properties of different vocabularies or other patterns in the knowledge base. This process can be accelerated and made more efficient using a set of query templates that extract statistics about the knowledge base or the patterns of vocabulary usage. These query templates can extract both statistics that are generic such as the number of triples in the knowledge base, the number of instances of a specific class (e.g., the number of bus stops or the number of business places that belong to the category restaurant). In addition to finding defective patterns, by observing the evolution of these values after each new deployment, one can monitor the growth of the knowledge base.

Testers used Loupe to explore the statistics of the knowledge base and identify potential outliers. For example, all instances of a given class were analyzed for checking what the properties that were used with those instances and their cardinality are. Concretely, testers analyzed what is the cardinality of the geolocation properties associated with the instances of the dul: Place class that belong to the YelpBusiness category. Whilst exploring the data instances, testers identified that there are certain individuals with multiple geolocation properties or some individuals without any geolocation property even though the most common pattern is to have exactly one geolocation property. This indicated a quality problem in the knowledge base and the tester reported this to the developers to find the root cause of this inconsistency so that the developers can correct the errors in the knowledge base generation process.

MappingPedia A large volume of Semantic Web data is being generated from existing legacy datasets, for example, relational databases or CSV files. This data has to be transformed to RDF generally using mappings. W3C has standardised languages for mapping relational data and relational database schemas into RDF and OWL. The two main languages for mappings are the Direct Mapping (DM) [15] and the RDB to RDF Mapping Language (R2RML) [53]. There are other languages that 
extend DM and R2RML to fit other use cases, such as RML [61] and xR2RML [159]. Currently, a significant number of users have developed R2RML mappings, but they have been kept locally in each organization duplicating the effort needed to create mappings each time.

MappingPedia is a collaborative environment for the discovery, sharing, execution, and analysis of R2RML mappings. MappingPedia integrates several tools developed for the exploitation of RDF data. The core of the integrated tools is morph-RDB [191], an RDB2RDF engine that follows the R2RML specification. It supports two operational modes: (1) RDF data generation from a relational database or CSV file, and (2) SPARQL to SQL Query translation according to R2RML mapping descriptions. MIRROR [54] is a system that generates two sets of R2RML mappings: First, it creates a set of mappings similar to the W3C Direct Mappings ${ }^{2}$, and second, a set of R2RML mappings that result from the implicit knowledge encoded in relational database schemas.

In MappingPedia, the Loupe Linked Data profiling framework is used to perform inspections of the classes, properties and triples to gather explicit vocabulary, classes and property usage, and to discover implicit data patterns through a fine-grained set of metrics [192]. Such information can help to get an overview of the available mappings in Mappingpedia and the ontologies that are being used by those mappings. Furthermore, profiling information extracted by Loupe can be used for recommending mappings to be reused based on the vocabulary usage analysis of the mappings.

Common Quality Issues in Spanish DBpedia DBpedia data come from Wikipedia, the vast online encyclopedia that grows daily with the contributions of its editors. Until 2011 only data from the English language Wikipedia was extracted but, since then, several "local" DBpedia chapters were created for other Wikipedia languages. For the Spanish language, the Spanish DBpedia ${ }^{3}$ (esDBpedia) was created in 2012 and currently there are 15 other "local" DBpedia chapters. The Loupe Linked Data profiling framework was used to inspect the quality issues of the properties used in the Spanish DBpedia dataset according to conciseness, consistency, syntactic validity, and semantic accuracy quality dimensions.

The automatic generation of RDF data using the entities of the DBpedia ontology relies on the manual creation of mappings. In the case of the Spanish chapter of DBpedia, there have been three community events intended to show attendants the mapping process and to provide mappings for most used infobox templates ${ }^{4}$. Currently, there are around 100 mappings which cover more than $80 \%$ of the total data stored in the infoboxes of the Spanish Wikipedia.

\footnotetext{
2https://Www.w3 . org/TR/rdb-direct - mapping/

${ }^{3}$ http: //es.dbpedia.org

${ }^{4}$ http://mappings.dbpedia.org/server/statistics/es/
} 
However, many of these mappings have infobox fields that are neither related to DBpedia entities (properties or classes) nor to other ontology entities. In such cases, rather than ignoring these valuable data, the extraction process generates a triple relating the Wikipedia resource (as the subject), a property generated using the infobox field (as the predicate) and its value (as the object). On the one hand, the properties that are generated by the extractors using infobox fields can be distinguished because they have distinct namespace prefixes $(i . e$., either http://es.dbpedia.org/property/or http://dbpedia.org/property/). On the other hand, the DBpedia ontology properties have a different namespace ( http://dbpedia.org/ontology/).

Loupe was used to analyze the 37,857 distinct properties used in the Spanish DBpedia 2014 dataset in order to identify quality problems with respect to the conciseness, consistency, syntactic validity, and semantic accuracy quality dimensions [165]. More concretely, the analysis aimed at identifying the common patterns of quality problems in the dataset, the root-causes of those patterns, and potential means for minimizing or eliminating those identified quality problems. Furthermore, the statistical data for each ontology element (class or property) extracted from the Spanish DBpedia dataset are made available online ${ }^{5}$.

The study showed that $97.93 \%$ of the properties used in the Spanish DBpedia are auto-generated properties (not using the mappings in the DBpedia extraction process). The results showed that such properties suffer from conciseness quality issues due to several causes such as inconsistent capitalization (857 properties), inconsistent usage of accents (1,252 properties), slashes in infobox labels (107 properties), spelling mistakes, etc. We also found inconsistencies such as properties simultaneously being object and datatype properties (3,380 properties) or wrong domain/range values $(2,821$ properties). Syntactic validity problems were found in 3,675 properties.

The results of this analysis provided valuable feedback to the DBpedia community to understand the current quality issues in DBpedia and to improve the extraction process to eliminate some of the identified quality issues. Especially some of the issues found in the conciseness dimension can be eliminated by performing some pre-processing and cleaning of the infobox attributes before converting them into properties.

Ontology Evolution Analysis Loupe was used to perform an empirical analysis on how four well-known ontologies that were developed in a collaborative manner (DBpedia, Schema.org, PROV-O, and FOAF) to understand community-driven ontology evolution in practice. Loupe was used to analyse how those ontologies evolved through their lifetime and analyse the data quality issues caused by some of the

\footnotetext{
${ }^{5}$ http://loupe. linkeddata.es/loupe/
} 
ontology changes [170]. The study analysed the changes in those ontologies in the 53 versions of them examined in this study.

The elements that are subject to change in ontologies include concepts, properties, instances and axioms. These changes impact the entities that are described using these ontologies as well as any other ontologies that are dependent on the given ontology. In the ontology evolution topic, the research looked into several problems and challenging tasks. These problems include consistency maintenance, backward compatibility, ontology manipulation, understanding of ontology evolution, and change propagation. This study was focused on analyzing the changes in different versions of the ontology and on discussing their effect on data quality.

For discovering and extracting the versions of the different ontologies, we used LOV. LOV stores information about more than 500 vocabularies and several versions for each of them. We used the SPARQL querying facility in LOV to discover the versions of a given vocabulary and also to extract them. For the analysis, the Loupe Linked Data profiling framework was used. Loupe allows to easily analyze datasets by creating virtual SPARQL endpoints via a Dockerized Virtuoso instance and a set of parameterized SPARQL queries. In this study, Loupe for extracting all the information about classes, properties, and also subclass and subproperty relations in each of the vocabulary versions and also for comparing subsequent versions to identify classes and properties that are added or removed.

From the analysis carried out, the study suggested that there is a necessity of incorporation of a rigorous editorial process and governance to DBpedia as well as a set of tools to facilitate the proper evolution of the ontology. As DBpedia makes use of crowdsourcing at many different stages and includes people from different levels and areas of knowledge about the ontology, having a proper editorial process becomes very important.

Semantic Sensor Network Ontology Usage The W3C Spatial Data on the Web Interest Group carried out a study with the objective of analysing the usage of the Sensor, Observation, Sample, and Actuator (SOSA) ontology core and the Semantic Sensor Network (SSN) ontology. The analysis was carried out along two aspects: (a) usage in Linked Data datasets (Consumers) and (b) usage in ontologies that reuse or extend those ontologies (Producers). The Loupe Linked Data profiling framework was used in the former to extract the usage information.

The goals of the usage in Linked Data analysis were the following: a) to analyse which modules of the SSN ontology have been used in different datasets and b) to analyse the coverage of the SSN vocabulary terms and modules in those datasets. To this end, the Loupe Linked Data profiling framework was used for analysing the vocabulary usage of a large number of datasets.

The analysis was two-fold with automated analysis of datasets in the first stage 
and the manual inspection in the second stage. For the automatic analysis, the main data sources were used: (i) LOD Laundromat which includes datasets collected by catalog-specific scripts (e.g., the Datahub ones) and by hand, (ii) LOD Cloud Cache which includes crawled data from various RDF and Linked Data resources from around the Web, as well as data extracted using Virtuoso Sponger from web pages, and (iii) LODStats which includes the datasets from data catalogs such as data.gov, publicdata.eu and datahub.io.

In this task, the Loupe Linked Data profiling framework has been used to identify which vocabulary terms from the SSN ontology are used in each dataset. Loupe analyzed the vocabulary usage patterns stored in LOD Laundromat (657,902 datasets). Besides, LOD Cloud Cache (3,203,793 documents) and LODStats (2,005 datasets) have been analysed using SPARQL queries. The results of the study are reported as a W3C Note [92].

\subsubsection{RDF Shape Induction Method}

We have used RDF Shape induction for quality assessment and repair in several datasets and they are discussed in detail in Chapter 7. For understanding the practical use of the RDF Shape induction method, we have selected three different datasets based on the (i) popularity and representativeness in their domain: DBpedia for the encyclopedic domain, 3cixty Nice for the tourist and cultural domain, and BNE for the library domain; (ii) heterogeneity in terms of content being hosted such as periodic extraction of various event information collected in 3cixty Nice KB, encyclopedic information in DBpedia, and library records in BNE (iii) diversity in the update strategy: incremental and usually as batch for DBpedia and BNE, continuous update for 3cixty.

DBpedia $^{6}$ is among the most popular knowledge bases in the LOD cloud. This knowledge base is the output of the DBpedia project that was initiated by researchers from the Free University of Berlin and the University of Leipzig, in collaboration with OpenLink Software. DBpedia is roughly updated every year since the first public release in 2007. DBpedia is created from automatically extracted structured information contained in Wikipedia, ${ }^{7}$ such as infobox tables categorisation information, geo-coordinates, and external links. As we have discussed in Chapter 7

3Cixty is a knowledgebase describing cultural and tourist information concerning the cities of Milan and Nice. This knowledge base was initially developed within the 3Cixty project, ${ }^{8}$ which aimed to develop a semantic web platform to build realworld and comprehensive knowledge bases in the domain of culture and tourism for

\footnotetext{
${ }^{6}$ http://wiki.dbpedia.org

${ }^{7}$ https://Www. wikipedia.org

${ }^{8}$ https://www.3cixty.com
} 
cities. In detail, it contains descriptions of events and activities, places and sights, transportation facilities as well as social activities, collected from local and global data providers, and social media platforms.

Spanish National Library (BNE) dataset is a knowledge base that provides the information about bibliography catalogue from the Biblioteca Nacional de España (BNE, National Library of Spain) as Linked Data [225]. It contains more than 7 million entities from the bibliographic records. This dataset is generated by transforming records in MARC 21 format into RDF using vocabularies such as IFLA (International Federation of Library Associations) ontologies, RDA, and Dublin Core. 


\section{Bibliography}

[1] Ziawasch Abedjan and Felix Naumann. Improving rdf data through association rule mining. Datenbank-Spektrum, 13(2):111-120, Jul 2013. ISSN 1610-1995. doi: 10.1007/s13222-013-0126-x. URL https ://doi .org/10 . 1007/s13222 $013-0126-x$.

[2] Ziawasch Abedjan, Toni Gruetze, Anja Jentzsch, and Felix Naumann. Profiling and mining RDF data with ProLOD++. In Proceedings of the IEEE 30th International Conference on Data Engineering (ICDE 2014), pages 1198-1201. IEEE, 2014.

[3] Ziawasch Abedjan, Lukasz Golab, and Felix Naumann. Profiling relational data: a survey. The VLDB Journal, 24(4):557-581, 2015. ISSN 0949-877X. doi: 10. 1007/s00778-015-0389-y. URL http://dx. doi.org/10 . 1007/s00778-015$0389-\mathrm{y}$.

[4] Andrejs Abele. Linked data profiling: Identifying the domain of datasets based on data content and metadata. In Proceedings of the 25th International Conference Companion on World Wide Web, pages 287-291, 2016.

[5] Maribel Acosta, Amrapali Zaveri, Elena Simperl, Dimitris Kontokostas, Sören Auer, and Jens Lehmann. Crowdsourcing Linked Data quality assessment. In H. Alani, L. Kagal, A. Fokoue, P. Groth, C. Biemann, J.X. Parreira, L. Aroyo, N. Noy, C. Welty, and K. Janowicz, editors, Proceedings of the 12th International Semantic Web Conference, Sydney, NSW, Australia, pages 260-276. Springer, 2013.

[6] Rakesh Agrawal, Tomasz Imieliński, and Arun Swami. Mining association rules between sets of items in large databases. In Acm sigmod record, volume 22, pages 207-216. ACM, 1993.

[7] Rakesh Agrawal, Heikki Mannila, Ramakrishnan Srikant, Hannu Toivonen, A Inkeri Verkamo, et al. Fast discovery of association rules. Advances in knowledge discovery and data mining, 12(1):307-328, 1996. 
[8] H. Al-Kilidar, K. Cox, and B. Kitchenham. The use and usefulness of the ISO/IEC 9126 quality standard. In Proceedings of the 2005 International Symposium on Empirical Software Engineering, Noosa Heads, Australia, pages 126-132. 2005.

[9] Gray Alasdair, Joachim Baran, Marshall M. Scott, and Dumontier Michel. Dataset Descriptions: HCLS Community Profile. https : //www.w3.org/TR/ hcls-dataset/, 2015. [Online; accessed 17-March-2017].

[10] Riccardo Albertoni and Antoine Isaac. Data on the web best practices: Data quality vocabulary. W3C recommendation, 2016.

[11] Riccardo Albertoni, Monica De Martino, and Paola Podesta. A linkset quality metric measuring multilingual gain in SKOS thesauri. In Anisa Rula, Amrapali Zaveri, Magnus Knuth, and Dimitris Kontokostas, editors, Proceedings of the 2nd Workshop on Linked Data Quality (LDQ2015). Co-located with 12th Extended Semantic Web Conference (ESWC 2015) . Portorož, Slovenia, 2015.

[12] Hunt Allcott and Matthew Gentzkow. Social Media and Fake News in the 2016 Election. Technical report, National Bureau of Economic Research, 2017.

[13] Mona Alshahrani, Mohammad Asif Khan, Omar Maddouri, Akira R Kinjo, Núria Queralt-Rosinach, and Robert Hoehndorf. Neuro-symbolic representation learning on biological knowledge graphs. Bioinformatics, 33(17):2723-2730, 2017.

[14] Gabor Angeli, Melvin Jose Johnson Premkumar, and Christopher D Manning. Leveraging linguistic structure for open domain information extraction. In Proceedings of the 53rd Annual Meeting of the Association for Computational Linguistics and the 7th International Joint Conference on Natural Language Processing (Volume 1: Long Papers), volume 1, pages 344-354, 2015.

[15] Marcelo Arenas, Alexandre Bertails, Eric Prud'hommeaux, and Juan Sequeda. A Direct Mapping of Relational Data to RDF, September 2012. URL https: //wWw.w3 . org/TR/rdb-direct-mapping/.

[16] G Atemezing, Fabien Gandon, Gabriel Kepeklian, François Scharffe, Raphaël Troncy, B Vatant, and S Villata. Publishing Linked Data Requires More than Just Using a Tool. In W3C 2013, Workshop on Open Data on the Web, 2013.

[17] Sören Auer, Christian Bizer, Georgi Kobilarov, Jens Lehmann, Richard Cyganiak, and Zachary Ives. DBpedia: A Nucleus for a Web of Open Data. The Semantic Web, pages 722-735, 2007. 
[18] Sören Auer, Jens Lehmann, and Axel-Cyrille Ngonga Ngomo. Introduction to linked data and its lifecycle on the web. In Reasoning Web. Semantic Technologies for the Web of Data, pages 1-75. Springer, 2011.

[19] Motoei Azuma. SQuaRE: The next generation of the ISO/IEC 9126 and 14598 international standards series on software product quality. In Katrina Maxwell, Serge Oligny, Rob Kusters, and Erik van Veenendaal, editors, Proceedings of the 12th European Software Control and Metrics Conference (ESCOM). London, UK, pages 337-346, 2001.

[20] Franz Baader. The description logic handbook: Theory, implementation and applications. Cambridge university press, 2003.

[21] Michele Banko, Michael J Cafarella, Stephen Soderland, Matthew Broadhead, and Oren Etzioni. Open information extraction from the web. In IJCAI, volume 7, pages 2670-2676, 2007.

[22] Adrien Basse, Fabien Gandon, Isabelle Mirbel, and Moussa Lo. Dfs-based frequent graph pattern extraction to characterize the content of rdf triple stores. In Web Science Conference 2010 (WebSci10), 2010.

[23] Dave Beckett and Brian McBride. RDF/XML syntax specification (revised). W3C recommendation, 2004.

[24] Wouter Beek, Laurens Rietveld, Hamid R Bazoobandi, Jan Wielemaker, and Stefan Schlobach. Lod laundromat: a uniform way of publishing other peopleâĂŹs dirty data. In International Semantic Web Conference, pages 213-228. Springer, 2014.

[25] B. Behkamal, M. Kahani, and M.K. Akbari. Customizing ISO 9126 quality model for evaluation of B2B applications. Information and software technology, 51(3):599-609, 2009. ISSN 0950-5849.

[26] Behshid Behkamal, Mohsen Kahani, and Ebrahim Bagheri. Quality Metrics for Linked Open Data. In Qiming Chen, Abdelkader Hameurlain, Farouk Toumani, Roland Wagner, and Hendrik Decker, editors, Proceedings of the 26th International Conference on Database and Expert Systems Applications, volume 9261, pages 144-152. Springer, 2015. doi: http://dx.doi.org/10.1007/978-3-31922849-5_11.

[27] François Belleau, Marc-Alexandre Nolin, Nicole Tourigny, Philippe Rigault, and Jean Morissette. Bio2rdf: towards a mashup to build bioinformatics knowledge systems. Journal of biomedical informatics, 41(5):706-716, 2008. 
[28] Mohamed Ben Ellefi, Zohra Bellahsene, John G Breslin, Elena Demidova, Stefan Dietze, Julian Szymański, and Konstantin Todorov. Rdf dataset profiling-a survey of features, methods, vocabularies and applications. Semantic Web, 9 (5):677-705, 2018.

[29] Salima Benbernou and Mourad Ouziri. Enhancing data quality by cleaning inconsistent big rdf data. In 2017 IEEE International Conference on Big Data (Big Data), pages 74-79. IEEE, 2017.

[30] Tim Berners-Lee, James Hendler, and Ora Lassila. The Semantic Web. Scientific American, 284(5):34-43, 2001.

[31] Manuel F Bertoa, José María Troya, and Antonio Vallecillo. Measuring the usability of software components. Journal of Systems and Software, 79(3): 427-439, 2006.

[32] Christian Bizer and Richard Cyganiak. Quality-driven information filtering using the WIQA policy framework. Web Semantics: Science, Services and Agents on the World Wide Web, 7(1):1-10, 2009.

[33] Christian Bizer, Tom Heath, and Tim Berners-Lee. Linked Data: The story so far. In Semantic services, interoperability and web applications: emerging concepts, pages 205-227. IGI Global, 2011.

[34] Jens Bleiholder and Felix Naumann. Data fusion. ACM computing surveys (CSUR), 41(1):1-41, 2009.

[35] Peter Bloem and Gerben K. D. De Vries. Machine learning on linked data, a position paper. In Proceedings of the 1st International Conference on Linked Data for Knowledge Discovery - Volume 1232, LD4KD'14, pages 64-68, Aachen, Germany, Germany, 2014. CEUR-WS.org. URL http://dl . acm . org/citation . cfm?id=3053827. 3053834.

[36] Eva Blomqvist, Ziqi Zhang, Anna Lisa Gentile, Isabelle Augenstein, and Fabio Ciravegna. Statistical knowledge patterns for characterising linked data. In Proceedings of the 4th International Conference on Ontology and Semantic Web Patterns-Volume 1188, pages 1-13. CEUR-WS. org, 2013.

[37] Christoph Böhm, Felix Naumann, Ziawasch Abedjan, Dandy Fenz, Toni Grütze, Daniel Hefenbrock, Matthias Pohl, and David Sonnabend. Profiling Linked Open Data with ProLOD. In Proceedings of the 26th International Conference on Data Engineering Workshops (ICDEW), Long Beach, California, USA, pages 175-178. IEEE, 2010. 
[38] Kurt Bollacker, Colin Evans, Praveen Paritosh, Tim Sturge, and Jamie Taylor. Freebase: a collaboratively created graph database for structuring human knowledge. In Proceedings of the 2008 ACM SIGMOD international conference on Management of data, pages 1247-1250, 2008.

[39] Piero A Bonatti, Aidan Hogan, Axel Polleres, and Luigi Sauro. Robust and scalable linked data reasoning incorporating provenance and trust annotations. Web Semantics: Science, Services and Agents on the World Wide Web, 9(2): 165-201, 2011.

[40] Iovka Boneva, Jérémie Dusart, Daniel Fernández Alvarez, and Jose Emilio Labra Gayo. Semi automatic construction of shex and shacl schemas. arXiv preprint arXiv:190\%.10603, 2019.

[41] Iovka Boneva, Jérémie Dusart, Daniel Fernándezálvarez, and Jose Emilio Labra Gayo. Shape designer for shex and shacl constraints. Posters and Demonstrations at the 18th International Semantic Web Conference (ISWC 2019), 2019.

[42] Leo Breiman. Random forests. Machine learning, 45(1):5-32, 2001.

[43] Dan Brickley and R.V. Guha. RDF Schema 1.1, February 2014. URL https: //www.w3. org/TR/rdf-schema/.

[44] Martin Brümmer, Ciro Baron, Ivan Ermilov, Markus Freudenberg, Dimitris Kontokostas, and Sebastian Hellmann. DataID: Towards Semantically Rich Metadata for Complex Datasets. In Proceedings of the 10th International Conference on Semantic Systems, SEM '14, pages 84-91. ACM, 2014. ISBN 978-1-4503-2927-9. doi: 10.1145/2660517.2660538.

[45] Lorenz Bühmann, Daniel Fleischhacker, Jens Lehmann, Andre Melo, and Johanna Völker. Inductive lexical learning of class expressions. In International Conference on Knowledge Engineering and Knowledge Management, pages 42-53. Springer, 2014.

[46] Carlos Buil-Aranda, Aidan Hogan, Jürgen Umbrich, and Pierre-Yves Vandenbussche. Sparql web-querying infrastructure: Ready for action? In International Semantic Web Conference, pages 277-293. Springer, 2013.

[47] Cody Burleson, Miguel Esteban-Gutiérrez, and Nandana Mihindukulasooriya. Linked Data Platform Best Practices and Guidelines, August 2014. URL http : //www.w3 . org/TR/ldp - bp/. 
[48] Andrew Carlson, Justin Betteridge, Bryan Kisiel, Burr Settles, Estevam R Hruschka Jr, and Tom M Mitchell. Toward an architecture for never-ending language learning. In $A A A I$, volume 5, page 3. Atlanta, 2010.

[49] Nitesh V Chawla, Kevin W Bowyer, Lawrence O Hall, and W Philip Kegelmeyer. Smote: synthetic minority over-sampling technique. Journal of artificial intelligence research, 16:321-357, 2002.

[50] Ping Chen and Walter Garcia. Hypothesis generation and data quality assessment through association mining. In Cognitive Informatics (ICCI), 2010 9th IEEE International Conference on, pages 659-666. IEEE, 2010.

[51] M Chisholm. The Implementation of Basel Committee BCBS 239: An IndustryWide Challenge for International Data Management. In Proceedings of the 2nd International Data and Information Management Conference (IDIMC), Loughborough, UK, 2014.

[52] Richard Cyganiak, David Wood, and Markus Lanthaler. RDF 1.1 Concepts and Abstract Syntax, February 2014. URL https://www.w3.org/TR/rdf11concepts/.

[53] Souripriya Das, Seema Sundara, and Richard Cyganiak. R2RML: RDB to RDF Mapping Language, September 2012. URL https : //www.w3 . org/TR/r2 rml/.

[54] Luciano Frontino de Medeiros, Freddy Priyatna, and Óscar Corcho. MIRROR: automatic R2RML mapping generation from relational databases. In Engineering the Web in the Big Data Era, ICWE'15, 2015.

[55] Luc De Raedt, Tias Guns, and Siegfried Nijssen. Constraint programming for data mining and machine learning. In Proceedings of the Twenty-Fourth AAAI Conference on Artificial Intelligence (AAAI-10), pages 1671-1675, 2010.

[56] Jeremy Debattista, Christoph Lange, and Sören Auer. daQ, an Ontology for Dataset Quality Information. In Christian Bizer, Tom Heath, Sören Auer, and Tim Berners-Lee, editors, Proceedings of the Workshop on Linked Data on the Web (LDOW2014). Co-located with the 23rd International World Wide Web Conference (WWW2014), Seoul, Korea, 2014.

[57] Jeremy Debattista, Sören Auer, and Christoph Lange. Luzzu - A Framework for Linked Data quality assessment. In Proceedings of IEEE Tenth International Conference on Semantic Computing (ICSC2016), pages 124-131, 2016. 
[58] Jeremy Debattista, Christoph Lange, Sören Auer, and Dominic Cortis. Evaluating the quality of the lod cloud: an empirical investigation. Semantic Web, 9 (6):859-901, 2018.

[59] Jeremy Debattista, Judie Attard, Rob Brennan, and Declan O'Sullivan. Is the lod cloud at risk of becoming a museum for datasets? looking ahead towards a fully collaborative and sustainable lod cloud. In Companion Proceedings of The 2019 World Wide Web Conference, pages 850-858, 2019.

[60] Jacob Devlin, Ming-Wei Chang, Kenton Lee, and Kristina Toutanova. BERT: Pre-training of deep bidirectional transformers for language understanding. In Proceedings of the 2019 Conference of the North American Chapter of the Association for Computational Linguistics: Human Language Technologies, Volume 1 (Long and Short Papers), pages 4171-4186, Minneapolis, Minnesota, June 2019. Association for Computational Linguistics. doi: 10.18653/v1/N191423. URL https://www. aclweb.org/anthology/N19-1423.

[61] Anastasia Dimou, Miel Vander Sande, Pieter Colpaert, Ruben Verborgh, Erik Mannens, and Rik Van de Walle. RML: A Generic Language for Integrated RDF Mappings of Heterogeneous Data. In Proceedings of the Workshop on Linked Data on the Web (LDOW 2014), pages 1-5, 2014.

[62] Anastasia Dimou, Dimitris Kontokostas, Markus Freudenberg, Ruben Verborgh, Jens Lehmann, Erik Mannens, Sebastian Hellmann, and Rik Van de Walle. Assessing and refining mappingsto rdf to improve dataset quality. In International Semantic Web Conference, pages 133-149. Springer, 2015.

[63] Anastasia Dimou, Dimitris Kontokostas, Markus Freudenberg, Ruben Verborgh, Jens Lehmann, Erik Mannens, Sebastian Hellman, and Rik Van de Walle. Dbpedia mappings quality assessment. In 15th International Semantic Web Conference, pages 1-4. CEUR-WS, 2016.

[64] Xin Luna Dong and Divesh Srivastava. Big data integration. In Data Engineering (ICDE), 2013 IEEE 29th International Conference on, pages 1245-1248. IEEE, 2013.

[65] R.G. Dromey. Software product quality: theory, model, and practice. Technical report, Software Quality Institute, Brisbane, Australia, 1998.

[66] M. Duerst and M. Suignard. Internationalized Resource Identifiers (IRIs), January 2005. URL https : //www.w3 . org/TR/rdf11-concepts/. 
[67] Suzanne M Embury, Binling Jin, Sandra Sampaio, and Iliada Eleftheriou. On the Feasibility of Crawling Linked Data Sets for Reusable Defect Corrections. In Proceedings of the 1st Workshop on Linked Data Quality (LDQ2014), 2014.

[68] Orri Erling and Ivan Mikhailov. Virtuoso: RDF Support in a Native RDBMS, pages 501-519. Springer Berlin Heidelberg, Berlin, Heidelberg, 2010. ISBN 978-3-642-04329-1. doi: 10.1007/978-3-642-04329-1_21. URL https://doi. org/10 . 1007/978- 3-642-04329-1_21.

[69] Ivan Ermilov, Michael Martin, Jens Lehmann, and Sören Auer. Linked Open Data statistics: Collection and exploitation. In Pavel Klinov and Dmitry Mouromtsev, editors, Proceedings of the 4 th International Conference on Knowledge Engineering and the Semantic Web (KESW2013), Saint Petersburg, Russia. Communications in Computer and Information Science, Volume 394, pages 242-249. Springer, 2013.

[70] Ivan Ermilov, Jens Lehmann, Michael Martin, editor="Groth Paul Auer, Sören", Elena Simperl, Alasdair Gray, Marta Sabou, Markus Krötzsch, Freddy Lecue, Fabian Flöck, and Yolanda Gil. LODStats: The Data Web Census Dataset, pages 38-46. Springer International Publishing, Cham, 2016. ISBN 978-3-319-46547-0. doi: 10.1007/978-3-319-46547-0_5. URL http : //dx . doi .org/10. 1007/9783-319-46547- - -5 .

[71] Ivan Ermilov, Jens Lehmann, Michael Martin, editor="Groth Paul Auer, Sören", Elena Simperl, Alasdair Gray, Marta Sabou, Markus Krötzsch, Freddy Lecue, Fabian Flöck, and Yolanda Gil. LODStats: The Data Web Census Dataset, pages 38-46. Springer International Publishing, Cham, 2016. ISBN 978-3-319-46547-0. doi: 10.1007/978-3-319-46547-0_5. URL http : //dx . doi . org/10 . 1007/9783 - 319 - 46547 - 0 -5.

[72] Oren Etzioni, Anthony Fader, Janara Christensen, Stephen Soderland, et al. Open information extraction: The second generation. In Twenty-Second International Joint Conference on Artificial Intelligence, 2011.

[73] Wenfei Fan and Floris Geerts. Foundations of data quality management. Synthesis Lectures on Data Management, 4(5):1-217, 2012.

[74] Kevin Chekov Feeney, Declan O'Sullivan, Wei Tai, and Rob Brennan. Improving curated web-data quality with structured harvesting and assessment. International Journal on Semantic Web and Information Systems (IJSWIS), 10(2):35-62, 2014. 
[75] Javier D Fernández, Miguel A Martínez-Prieto, Pablo de la Fuente Redondo, and Claudio Gutiérrez. Characterising rdf data sets. Journal of Information Science, 44(2):203-229, 2018.

[76] Javier David Fernandez Garcia, Wouter Beek, Miguel A Martínez-Prieto, and Mario Arias. Lod-a-lot: A queryable dump of the lod cloud. 2017.

[77] Philippe Flajolet. On adaptive sampling. Computing, 43(4):391-400, 1990.

[78] Philippe Flajolet and G Nigel Martin. Probabilistic counting algorithms for data base applications. Journal of computer and system sciences, 31(2):182-209, 1985.

[79] Daniel Fleischhacker, Johanna Völker, and Heiner Stuckenschmidt. Mining rdf data for property axioms. In On the Move to Meaningful Internet Systems: OTM 2012, pages 718-735, Berlin, Heidelberg, 2012. Springer Berlin Heidelberg.

[80] Daniel Fleischhacker, Heiko Paulheim, Volha Bryl, Johanna Völker, and Christian Bizer. Detecting errors in numerical linked data using cross-checked outlier detection. In International Semantic Web Conference, pages 357-372. Springer, 2014.

[81] Annika Flemming. Quality characteristics of linked data publishing datasources. Master's thesis, Humboldt-Universität of Berlin, 2010.

[82] Benedikt Forchhammer, Anja Jentzsch, and Felix Naumann. LODOP-MultiQuery Optimization for Linked Data Profiling Queries. In In Proceedings of the 1st International Workshop on Dataset Profiling, 2014.

[83] X. Franch and J.P. Carvallo. Using quality models in software package selection. Software, IEEE, 20(1):34-41, 2003. ISSN 0740-7459.

[84] David A Freedman. Statistical models: theory and practice. cambridge university press, 2009.

[85] Thomas MJ Fruchterman and Edward M Reingold. Graph drawing by forcedirected placement. Software: Practice and experience, 21(11):1129-1164, 1991.

[86] Christian Fürber and Martin Hepp. SWIQA - A Semantic Web information quality assessment framework. In Proceedings of the 19th European Conference on Information Systems (ECIS 2011), volume 15, page 19, 2011.

[87] Christian Fürber and Martin Hepp. Towards a vocabulary for data quality management in Semantic Web architectures. In Roberto De Virgilio, Devis 
Bianchini, Valeria De Antonellis, Kjell Orsborn, and Silvia Stefanova, editors, Proceedings of the 1st International Workshop on Linked Web Data Management, Uppsala, Sweden, pages 1-8. ACM, 2011. doi: http://dx.doi.org/10.1145/ 1966901.1966903.

[88] Johannes Fürnkranz and Peter A Flach. Roc âĂŸnâĂŹrule learningâĂ ̌̌towards a better understanding of covering algorithms. Machine Learning, 58(1):39-77, 2005 .

[89] Matthew Gamble and Carole Goble. Quality, trust, and utility of scientific data on the web: Towards a joint model. In Proceedings of the 3rd international web science conference, page 15. ACM, 2011.

[90] Aldo Gangemi, Valentina Presutti, Diego Reforgiato Recupero, Andrea Giovanni Nuzzolese, Francesco Draicchio, and Misael Mongiovã ČǍň. Semantic Web Machine Reading with FRED. Semantic Web, 8(6):873-893, 2017.

[91] Raúl García-Castro. Benchmarking Semantic Web technology. Studies on the Semantic Web vol. 3. AKA Verlag - IOS Press., 2010.

[92] Raúl García-Castro, Armin Haller, and Nandana Mihindukulasooriya. On the usage of the SSN ontology, May 2018. URL https://w3c.github.io/sdw/ ssn-usage/.

[93] Jose Emilio Labra Gayo, Eric Prud'Hommeaux, Iovka Boneva, and Dimitris Kontokostas. Validating rdf data. Synthesis Lectures on Semantic Web: Theory and Technology, 7(1):1-328, 2017.

[94] Lise Getoor and Ben Taskar. Introduction to statistical relational learning. MIT press, 2007.

[95] Yolanda Gil and Donovan Artz. Towards content trust of web resources. Web Semantics: Science, Services and Agents on the World Wide Web, 5(4):227-239, 2007.

[96] Yolanda Gil and Varun Ratnakar. Trusting information sources one citizen at a time. In Ian Horrocks and James Hendler, editors, Proceedings of the 1st International Semantic Web Conference, Sardinia, Italy, pages 162-176. Springer, 2002.

[97] Hugh Glaser, Afraz Jaffri, and Ian Millard. Managing Co-reference on the Semantic Web. In Proceedings of the Linked Data on the Web Workshop (LDOW2009), pages 1-6, April 2009. 
[98] Jennifer Golbeck and Aaron Mannes. Using trust and provenance for content filtering on the semantic web. In $M T W, 2006$.

[99] Jennifer Golbeck, Bijan Parsia, and James Hendler. Trust Networks on the Semantic Web. In Cooperative Information Agents VII, volume 2782, pages 238-249. Springer Berlin Heidelberg, 2003.

[100] Tudor Groza, Anika Oellrich, and Nigel Collier. Using silver and semi-gold standard corpora to compare open named entity recognisers. In Bioinformatics and Biomedicine (BIBM), 2013 IEEE International Conference on, pages 481-485. IEEE, 2013.

[101] Christophe Guéret, Paul Groth, Claus Stadler, and Jens Lehmann. Assessing Linked Data mappings using network measures. In The Semantic Web: Research and Applications, pages 87-102. Springer, 2012.

[102] ZM Guo and AY Zhou. Research on Data Quality and Data Cleaning: A Survey. Journal of software, 13(11):2076-2082, 2002.

[103] Ben Hachey, Will Radford, Joel Nothman, Matthew Honnibal, and James R Curran. Evaluating entity linking with wikipedia. Artificial intelligence, 194: 130-150, 2013.

[104] A Hadhiatma. Improving data quality in the linked open data: a survey. In Journal of Physics: Conference Series, volume 978, page 012026. IOP Publishing, 2018.

[105] Steve Harris and Andy Seaborne. SPARQL 1.1 Query Language, March 2013. URL https://www.w3.org/TR/sparql11-query/.

[106] Olaf Hartig. Trustworthiness of data on the Web. In Proceedings of the STI Berlin \& CSW PhD Workshop. Citeseer, 2008.

[107] Sebastian Hellmann, Volha Bryl, Lorenz Bühmann, Milan Dojchinovski, Dimitris Kontokostas, Jens Lehmann, Uroš Milošević, Petar Petrovski, Vojtěch Svátek, Mladen Stanojević, et al. Knowledge base creation, enrichment and repair. In Linked Open Data-Creating Knowledge Out of Interlinked Data, pages 45-69. Springer, 2014.

[108] Stefan Heule, Marc Nunkesser, and Alexander Hall. Hyperloglog in practice: algorithmic engineering of a state of the art cardinality estimation algorithm. In Proceedings of the 16th International Conference on Extending Database Technology, pages 683-692. ACM, 2013. 
[109] Pascal Hitzler, Markus Krotzsch, Bijan Parsia, Peter F. Patel-Schneider, and Sebastian Rudolph. OWL 2 Web Ontology Language Primer, December 2012. URL https : //www.w3 . org/TR/2012/REC - owl2 - primer - 20121211/.

[110] Aidan Hogan, Andreas Harth, Alexandre Passant, Stefan Decker, and Axel Polleres. Weaving the Pedantic Web. In Proceedings of the Linked Data on the Web Workshop (LDOW2010), volume 628, 2010.

[111] Aidan Hogan, Jürgen Umbrich, Andreas Harth, Richard Cyganiak, Axel Polleres, and Stefan Decker. An empirical survey of linked data conformance. Web Semantics: Science, Services and Agents on the World Wide Web, 14:14-44, 2012 .

[112] Bernadette Hyland and David Wood. The Joy of Data - A Cookbook for Publishing Linked Government Data on the Web. In Linking government data, pages 3-26. Springer, 2011.

[113] ISO. ISO/IEC 15939:2007, Systems and software engineering - Measurement process. Technical report, International Organization for Standardization, 2007.

[114] ISO. ISO/IEC 25012:2008, Software engineering - Systems product Quality Requirements and Evaluation (SQuaRE) - Data quality model. Technical report, International Organization for Standardization, 2008.

[115] ISO. ISO/IEC 25010:2011, Systems and software engineering - Systems and software Quality Requirements and Evaluation (SQuaRE) - System and software quality models. Technical report, International Organization for Standardization, 2011.

[116] Subhi Issa, Onaopepo Adekunle, Fayçal Hamdia, Samira Si-said Cherfia, Michel Dumontierb, and Amrapali Zaverib. Linked data completeness: A systematic literature review. Semantic Web, pages 1-18, 2019.

[117] Subhi Issa, Fayçal Hamdi, and Samira Si-said Cherfi. Enhancing the conciseness of linked data by discovering synonym predicates. In International Conference on Knowledge Science, Engineering and Management, pages 739-750. Springer, 2019 .

[118] Juha Itkonen and Kristian Rautiainen. Exploratory testing: a multiple case study. In International Symposium on Empirical Software Engineering, 2005.

[119] Juha Itkonen, Mika V Mäntylä, and Casper Lassenius. Defect detection efficiency: Test case based vs. exploratory testing. In Empirical Software Engineering and Measurement, 200\%. ESEM 200\%. First International Symposium on, pages 61-70. IEEE, 2007. 
[120] Ian Jacobi, Lalana Kagal, and Ankesh Khandelwal. Rule-based trust assessment on the semantic web. In International Workshop on Rules and Rule Markup Languages for the Semantic Web, pages 227-241. Springer, 2011.

[121] Krzysztof Janowicz, Yingjie Hu, Grant McKenzie, Song Gao, Blake Regalia, Gengchen Mai, Rui Zhu, Benjamin Adams, and Kerry Taylor. Moon landing or safari? a study of systematic errors and their causes in geographic linked data. In The Annual International Conference on Geographic Information Science, pages 275-290. Springer, 2016.

[122] Alan Jeffrey and Peter F Patel-Schneider. Integrity constraints for linked data. In Proc. Int. Workshop Description Logics. Citeseer, 2011.

[123] Anja Jentzsch. Profiling the Web of Data. Proceedings of the 8th Ph.D. Retreat of the HPI Research school on Service-Oriented Systems Engineering, pages 107-117, 2014.

[124] Qiu Ji, Peter Haase, Guilin Qi, Pascal Hitzler, and Steffen Stadtmüller. RaDONâĂŤRepair and Diagnosis in Ontology Networks. In European Semantic Web Conference, pages 863-867. Springer, 2009.

[125] Saint John Walker. Big data: A revolution that will transform how we live, work, and think, 2014.

[126] Theodore Johnson. Data profiling. In LING LIU and M. TAMER ÖZSU, editors, Encyclopedia of Database Systems, pages 604-608, Boston, MA, 2009. Springer US. ISBN 978-0-387-39940-9. doi: 10.1007/978-0-387-39940-9_601. URL http://dx.doi.org/10.1007/978- 0-387-39940-9_601.

[127] Sujata Joshi and SR PriyankaShetty. Performance analysis of different classification methods in data mining for diabetes dataset using weka tool. International Journal on Recent and Innovation Trends in Computing and Communication, 3(3):1168-1173, 2015.

[128] Ademar Crotti Junior, Jeremy Debattista, and Declan OâĂŹSullivan. Assessing the Quality of R2RML Mappings. In Proceedings of the 1st International Workshop on Approaches for Making Data Interoperable, pages 1-12. 2019.

[129] Joseph M. Juran and A. Blanton Godfrey. Juran's Quality Handbook. Fifth Edition. McGraw-Hill, 2000.

[130] Stephen H Kan. Metrics and Models in Software Quality Engineering. AddisonWesley Longman Publishing Co., Inc., 2002. 
[131] Ning Kang, Erik M van Mulligen, and Jan A Kors. Training text chunkers on a silver standard corpus: can silver replace gold? BMC bioinformatics, 13(1): 17,2012 .

[132] Alexander Keith, Cyganiak Richard, Hausenblas Michael, and Zhao Jun. Describing Linked Datasets with the VoID Vocabulary (). W3C Interest Group Note, 2011. [Online; accessed 17-March-2017].

[133] Shahan Khatchadourian and Mariano P. Consens. Explod: Summary-based exploration of interlinking and rdf usage in the linked open data cloud. In The Semantic Web: Research and Applications, pages 272-287, Berlin, Heidelberg, 2010. Springer Berlin Heidelberg. ISBN 978-3-642-13489-0.

[134] Hassan Khosravi and Bahareh Bina. A survey on statistical relational learning. In Canadian Conference on AI, pages 256-268. Springer, 2010.

[135] B. Kitchenham. Procedures for performing systematic reviews. Technical report, Software Engineering Group, Department of Computer Science Keele University (UK) and Empirical Software Engineering, National ICT Australia, 2004.

[136] Barbara Kitchenham. DESMET: A method for evaluating software engineering methods and tools. Technical report, Department of Computer Science, University of Keele, Staffordshire, UK, 1996.

[137] Holger Knublauch and Dimitris Kontokostas. W3C Shapes Constraint Language (SHACL), July 2017. URL https://www.w3.org/TR/shacl/.

[138] Holger Knublauch, James Hendler, and Kingsley Idehen. SPARQL Inferencing Notation (SPIN), 2011. URL https://www.w3.org/Submission/2011/ SUBM-spin-overview-20110222/.

[139] Magnus Knuth, Dimitris Kontokostas, and Harald Sack. Linked Data Quality: Identifying and Tackling the Key Challenges. In Proceedings of the 1st Workshop on Linked Data Quality (LDQ2014), 2014.

[140] Daphne Koller and Nir Friedman. Probabilistic Graphical Models: Principles and Techniques - Adaptive Computation and Machine Learning. The MIT Press, 2009. ISBN 0262013193, 9780262013192.

[141] Dimitris Kontokostas, Amrapali Zaveri, Sören Auer, and Jens Lehmann. Triplecheckmate: A tool for crowdsourcing the quality assessment of linked data. In International Conference on Knowledge Engineering and the Semantic Web, pages 265-272. Springer, 2013. 
[142] Dimitris Kontokostas, Patrick Westphal, Sören Auer, Sebastian Hellmann, Jens Lehmann, Roland Cornelissen, and Amrapali Zaveri. Test-driven evaluation of Linked Data quality. In Proceedings of the 23rd International Conference on World Wide Web, pages 747-758. ACM, 2014.

[143] Andreas Langegger and Wolfram Woss. RDFStats - An Extensible RDF Statistics Generator and Library. In Proceedings of the 20th International Workshop on Database and Expert Systems Application, pages 79-83. IEEE, 2009.

[144] Andreas Langegger and Wolfram Woss. RDFStats âĂŞ An Extensible RDF Statistics Generator and Library. In 20th International Workshop on Database and Expert Systems Application, pages 79-83. IEEE, 2009.

[145] Rietveld Laurens, Wouter Beek, Rinkea Hoekstra, and Stefana Schlobach. Meta-data for a lot of LOD. Semantic Web Journal, 8(6):1067-1080, 2017.

[146] Yann LeCun, Yoshua Bengio, and Geoffrey Hinton. Deep learning. nature, 521 (7553):436-444, 2015.

[147] Jens Lehmann, Robert Isele, Max Jakob, Anja Jentzsch, Dimitris Kontokostas, Pablo N Mendes, Sebastian Hellmann, Mohamed Morsey, Patrick Van Kleef, Sören Auer, et al. DBpedia - A Large-scale, Multilingual Knowledge Base Extracted from Wikipedia. Semantic Web, 6(2):167-195, 2015.

[148] Yuangui Lei, Victoria Uren, and Enrico Motta. A framework for evaluating semantic metadata. In Proceedings of the 4 th international conference on Knowledge capture, pages 135-142. ACM, 2007.

[149] Huiying Li. Data Profiling for Semantic Web Data. In Web Information Systems and Mining, pages 472-479, Berlin, Heidelberg, 2012. Springer Berlin Heidelberg. ISBN 978-3-642-33469-6.

[150] Yinhan Liu, Myle Ott, Naman Goyal, Jingfei Du, Mandar Joshi, Danqi Chen, Omer Levy, Mike Lewis, Luke Zettlemoyer, and Veselin Stoyanov. Roberta: A robustly optimized bert pretraining approach. ArXiv, abs/1907.11692, 2019.

[151] Zhen Hua Liu and Dieter Gawlick. Management of Flexible Schema Data in RDBMSs-Opportunities and Limitations for NoSQL. In Conference on Innovative Data Systems Research (CIDR 2015), 2015.

[152] Fadi Maali and John Erickson. Data Catalog Vocabulary (DCAT). W3C recommendation, jan 2014. 
[153] Eetu Mäkelä. Aether - Generating and Viewing Extended VoID Statistical Descriptions of RDF Datasets, pages 429-433. Springer International Publishing, Cham, 2014. ISBN 978-3-319-11955-7. doi: 10.1007/978-3-319-11955-7_61. URL http : //dx.doi.org/10.1007/978-3-319-11955-7_61.

[154] Jiayuan Mao, Chuang Gan, Pushmeet Kohli, Joshua B Tenenbaum, and Jiajun $\mathrm{Wu}$. The neuro-symbolic concept learner: Interpreting scenes, words, and sentences from natural supervision. arXiv preprint arXiv:1904.12584, 2019.

[155] Andre Melo, Johanna Völker, and Heiko Paulheim. Type prediction in noisy rdf knowledge bases using hierarchical multilabel classification with graph and latent features. International Journal on Artificial Intelligence Tools, 26(02): 1760011, 2017.

[156] Pablo N. Mendes, Max Jakob, Andrés García-Silva, and Christian Bizer. DBpedia Spotlight: Shedding Light on the Web of Documents. In Proceedings of the rth International Conference on Semantic Systems, I-Semantics '11, pages 1-8, New York, NY, USA, 2011. ACM. ISBN 978-1-4503-0621-8.

[157] Pablo N Mendes, Hannes Mühleisen, and Christian Bizer. Sieve: Linked Data quality assessment and fusion. In Proceedings of the 2012 Joint EDBT/ICDT Workshops, pages 116-123. ACM, 2012.

[158] Robert Meusel and Heiko Paulheim. Heuristics for fixing common errors in deployed schema. org microdata. In European Semantic Web Conference, pages 152-168. Springer, 2015.

[159] Franck Michel, Loïc Djimenou, Catherine Faron-Zucker, and Johan Montagnat. Translation of relational and non-relational databases into RDF with xR2RML. In International Conference on Web Information Systems and Technologies WEBIST'15, 2015.

[160] Nandana Mihindukulasooriya and Roger Menday. Linked Data Platform 1.0 Primer, April 2015. URL http://www.w3.org/TR/ldp-primer/.

[161] Nandana Mihindukulasooriya, Raúl García-Castro, and Miguel EstebanGutiérrez. Linked Data Platform as a novel approach for Enterprise Application Integration. In Proceedings of the 4th International Workshop on Consuming Linked Data (COLD2013), Sydney, Australia, Oct 2013.

[162] Nandana Mihindukulasooriya, Raul Garcia-Castro, and Miguel Esteban GutiÂlrrez. Linked data platform as a novel approach for enterprise application integration. In Olaf Hartig, Juan F. Sequeda, Aidan Hogan, and Takahide 
Matsutsuka, editors, COLD, volume 1034 of CEUR Workshop Proceedings. CEUR-WS.org, 2013. URL http://dblp.uni-trier.de/db/conf/semweb/ cold2013.html\#MihindukulasooriyaGG13.

[163] Nandana Mihindukulasooriya, María Poveda-Villalón, Raúl García-Castro, and Asunción Gómez-Pérez. Loupe-An Online Tool for Inspecting Datasets in the Linked Data Cloud. In Demo at the 14th International Semantic Web Conference, Bethlehem, USA, 2015.

[164] Nandana Mihindukulasooriya, Mariano Rico, Raúl García-Castro, and Asunción Gómez-Pérez. An analysis of the quality issues of the properties available in the spanish dbpedia. In Conference of the Spanish Association for Artificial Intelligence, pages 198-209. Springer, 2015.

[165] Nandana Mihindukulasooriya, Mariano Rico, Raúl García-Castro, and Asunción" Gómez-Pérez. An analysis of the quality issues of the properties available in the spanish dbpedia. In Advances in Artificial Intelligence, pages 198-209, Cham, 2015. Springer International Publishing. ISBN 978-3-319-24598-0.

[166] Nandana Mihindukulasooriya, Raúl García-Castro, and Asunción Gómez-Pérez. Ld sniffer: a quality assessment tool for measuring the accessibility of linked data. In European Knowledge Acquisition Workshop, pages 149-152. Springer, 2016 .

[167] Nandana Mihindukulasooriya, María Poveda-Villalón, Raúl García-Castro, and Asunción Gómez-Pérez. An RDF Dataset Description Model for Expressing Vocabulary Usage Patterns. In Proceedings of the W3C Smart Descriptions Smarter Vocabularies Workshop (SDSVoc 2016), pages 1-4. W3C, 2016.

[168] Nandana Mihindukulasooriya, Giuseppe Rizzo, Raphaël Troncy, Oscar Corcho, and Raul Garcia Castro. A two-fold quality assurance approach for dynamic knowledge bases: The 3cixty use case. In International Workshop on Completing and Debugging the Semantic Web (CoDeS'16), May 30, 2016, Heraklion, Greece, Heraklion, GRĖCE, 05 2016. URL http: //ceur-ws . org/Vol - 1586/codes2. pdf.

[169] Nandana Mihindukulasooriya, Raúl García-Castro, Freddy Priyatna, Edna Ruckhaus, and Nelson Saturno. A linked data profiling service for quality assessment. In The Semantic Web: ESWC 2017 Satellite Events, pages 335340, Cham, 2017. Springer International Publishing. ISBN 978-3-319-70407-4.

[170] Nandana Mihindukulasooriya, María Poveda-Villalón, Raúl García-Castro, and Asunción Gómez-Pérez. Collaborative ontology evolution and data quality - an 
empirical analysis. In OWL: Experiences and Directions - Reasoner Evaluation, pages 95-114, Cham, 2017. Springer International Publishing. ISBN 978-3-31954627-8.

[171] Nandana Mihindukulasooriya, Mariano Rico, Idafen Santana-Pérez, Raúl García-Castro, and Asunción Gómez-Pérez. Repairing hidden links in linked data: Enhancing the quality of rdf knowledge graphs. In Proceedings of the Knowledge Capture Conference, pages 1-8, 2017.

[172] Mike Mintz, Steven Bills, Rion Snow, and Dan Jurafsky. Distant supervision for relation extraction without labeled data. In Proceedings of the Joint Conference of the 47th Annual Meeting of the ACL and the 4th International Joint Conference on Natural Language Processing of the AFNLP: Volume 2-Volume 2, pages 1003-1011. Association for Computational Linguistics, 2009.

[173] Mehryar Mohri, Afshin Rostamizadeh, and Ameet Talwalkar. Foundations of machine learning. MIT press, 2012.

[174] Mir-Abolfazl Mostafavi, Geoffrey Edwards, and Robert Jeansoulin. An ontologybased method for quality assessment of spatial data bases. In Proc. of the Third International Symposium on Spatial Data Quality, pages 49-66, 2004.

[175] Hannes Mühleisen and Christian Bizer. Web Data Commons-Extracting Structured Data from Two Large Web Corpora. Linked Data on the Web (LDOW 2012), 937:133-145, 2012.

[176] Heiko Müller and Johann-Christph Freytag. Problems, methods, and challenges in comprehensive data cleansing. Professoren des Inst. Für Informatik, 2005.

[177] Sergio Munoz, Jorge Pérez, and Claudio Gutierrez. Minimal Deductive Systems for RDF. In European Semantic Web Conference, pages 53-67. Springer, 2007.

[178] Felix Naumann. Data profiling revisited. ACM SIGMOD Record, 42(4):40-49, 2014 .

[179] Victoria Nebot and Rafael Berlanga. Finding association rules in semantic web data. Knowledge-Based Systems, 25(1):51-62, 2012.

[180] Thomas Neumann and Guido Moerkotte. Characteristic sets: Accurate cardinality estimation for rdf queries with multiple joins. In Data Engineering (ICDE), 2011 IEEE 27th International Conference on, pages 984-994. IEEE, 2011. 
[181] JS Oakland and AJ Aldridge. Quality management in civil and structural engineering consulting. International Journal of Quality \& Reliability Management, 1995.

[182] OMB. Guidelines for ensuring and maximizing the quality, objectivity, utility, and integrity of information disseminated by federal agencies. Part IX. Office of Management and Budget. Technical report, Office of Management and Budged (USA), 2002.

[183] Matteo Palmonari, Anisa Rula, Riccardo Porrini, Andrea Maurino, Blerina Spahiu, and Vincenzo Ferme. ABSTAT: Linked Data summaries with ABstraction and STATistics. In The Semantic Web: ESWC 2015 Satellite Events, pages 128-132. Springer, 2015.

[184] Peter F Patel-Schneider. Using description logics for rdf constraint checking and closed-world recognition. In Twenty-Ninth AAAI Conference on Artificial Intelligence, 2015.

[185] Peter F Patel-Schneider. Ashacl: Alternative shapes constraint language. arXiv preprint arXiv:1702.01795, 2017.

[186] Heiko Paulheim. Knowledge graph refinement: A survey of approaches and evaluation methods. Semantic web, 8(3):489-508, 2017.

[187] Heiko Paulheim and Christian Bizer. Type Inference on Noisy RDF Data. In International semantic web conference, pages 510-525. Springer, 2013.

[188] Heiko Paulheim and Christian Bizer. Improving the quality of linked data using statistical distributions. International Journal on Semantic Web and Information Systems (IJSWIS), 10(2):63-86, 2014.

[189] Christoph Pinkel, Carsten Binnig, Ernesto Jiménez-Ruiz, Evgeny Kharlamov, Wolfgang May, Andriy Nikolov, Ana Sasa Bastinos, Martin G Skjæveland, Alessandro Solimando, Mohsen Taheriyan, et al. Rodi: Benchmarking relationalto-ontology mapping generation quality. Semantic Web, 9(1):25-52, 2018.

[190] Leo L Pipino, Yang W Lee, and Richard Y Wang. Data quality assessment. Communications of the ACM, 45(4):211-218, 2002.

[191] Freddy Priyatna, Óscar Corcho, and Juan Sequeda. Formalisation and experiences of R2RML-based SPARQL to SQL query translation using morph. In $W W W ' 14,2014$. 
[192] Freddy Priyatna, Edna Ruckhaus, Nandana Mihindukulasooriya, Oscar Corcho, and Nelson Saturno. MappingPedia: A Collaborative Environment for R2RML Mappings. In The Semantic Web: ESWC 2017 Satellite Events, pages 114-119, Cham, 2017. Springer International Publishing. ISBN 978-3-319-70407-4.

[193] Eric Prud'hommeaux, Jose Emilio Labra Gayo, and Harold Solbrig. Shape expressions: an rdf validation and transformation language. In Proceedings of the 10th International Conference on Semantic Systems, pages 32-40. ACM, 2014.

[194] Eric Prud'hommeaux, Iovka Boneva, Jose Emilio Labra-Gayo, and Gregg Kellogg. Shape Expressions Language 2.0, July 2017. URL http://shex. io/ shex-semantics/.

[195] Ross J. Quinlan. C4. 5: programs for machine learning. Elsevier, 2014. ISBN $1-55860-238-0$.

[196] Filip Radulovic, Raúl García-Castro, and Asunción Gómez-Pérez. SemQuaRE - An extension of the SQuaRE quality model for the evaluation of semantic technologies. Computer Standards \& Interfaces, 38:101-112, 2015.

[197] Thomas Rebele, Fabian Suchanek, Johannes Hoffart, Joanna Biega, Erdal Kuzey, and Gerhard Weikum. Yago: A multilingual knowledge base from wikipedia, wordnet, and geonames. In International Semantic Web Conference, pages 177-185. Springer, 2016.

[198] Raymond Reiter. On integrity constraints. In Proceedings of the 2nd Conference on Theoretical Aspects of Reasoning about Knowledge, pages 97-111. Morgan Kaufmann Publishers Inc., 1988.

[199] Mariano Rico, Nandana Mihindukulasooriya, and Asunción Gómez-Pérez. DataDriven RDF Property Semantic-Equivalence Detection Using NLP Techniques. In EKAW Proceedings, LNCS 10024, pages 797-804. Springer International Publishing, 2016. ISBN 978-3-319-49004-5. doi: 10.1007/978-3-319-49004-5_51. URL http://dx.doi.org/10.1007/978-3-319-49004-5_51.

[200] Mariano Rico, Nandana Mihindukulasooriya, Dimitris Kontokostas, Heiko Paulheim, Sebastian Hellmann, and Asunción Gómez-Pérez. Predicting incorrect mappings: a data-driven approach applied to dbpedia. In Proceedings of the 33rd annual ACM symposium on applied computing, pages 323-330, 2018.

[201] Laurens Rietveld, Wouter Beek, and Stefan Schlobach. LOD Lab: Experiments at LOD Scale. In International Semantic Web Conference, pages 339-355. Springer, 2015. 
[202] Petar Ristoski and Heiko Paulheim. Feature selection in hierarchical feature spaces. In International Conference on Discovery Science, pages 288-300. Springer, 2014.

[203] Dominique Ritze, Oliver Lehmberg, and Christian Bizer. Matching html tables to dbpedia. In Proceedings of the 5th International Conference on Web Intelligence, Mining and Semantics, pages 1-6, 2015.

[204] Dominique Ritze, Oliver Lehmberg, Yaser Oulabi, and Christian Bizer. Profiling the potential of web tables for augmenting cross-domain knowledge bases. In Proceedings of the 25th International Conference on World Wide Web, pages 251-261. International World Wide Web Conferences Steering Committee, 2016.

[205] G. Rizzo, R. Troncy, O. Corcho, A. Jameson, J. Plu, J.C. Ballesteros Hermida, A. Assaf, C. Barbu, A. Spirescu, K. Kuhn, I. Celino, R. Agarwal, C.K. Nguyen, A. Pathak, C. Scanu, M. Valla, T. Haaker, E.S. Verga, M. Rossi, and J.L. Redondo Garcia. 3cixty@Expo Milano 2015: Enabling Visitors to Explore a Smart City. In $14^{\text {th }}$ International Semantic Web Conference (ISWC), Semantic Web Challenge, 2015.

[206] Edna Ruckhaus, Oriana Baldizán, and María-Esther Vidal. Analyzing Linked Data quality with LiQuate. In On the Move to Meaningful Internet Systems: OTM 2013 Workshops, Graz, Austria, pages 629-638. Springer, 2013.

[207] Anisa Rula, Matteo Palmonari, and Andrea Maurino. Capturing the age of linked open data: Towards a dataset-independent framework. In Semantic Computing (ICSC), 2012 IEEE Sixth International Conference on, pages 218225. IEEE, 2012.

[208] Anisa Rula, Andrea Maurino, and Carlo Batini. Data quality issues in linked open data. In Data and information quality, pages 87-112. Springer, 2016.

[209] Arthur Ryman. Resource Shape 2.0, February 2014. URL https://www.w3. org/Submission/shapes/.

[210] Arthur G Ryman, Arnaud Le Hors, and Steve Speicher. Oslc resource shape: A language for defining constraints on linked data. Linked Data on the Web (LDOW2013), 996, 2013.

[211] Michael Schneider. OWL2 Web Ontology Language - RDF-Based Semantics, December 2012. URL http://wWw.w3.org/TR/owl-rdf-based-semantics.

[212] Saeedeh Shekarpour and SD Katebi. Modeling and evaluation of trust with an extension in semantic web. Journal of Web Semantics, 8(1):26-36, 2010. 
[213] Blerina Spahiu. Profiling Linked Data. PhD thesis, UNIVERSITY OF MILANO - BICOCCA, MILANO, 72017.

[214] Blerina Spahiu, Riccardo Porrini, Matteo Palmonari, Anisa Rula, and Andrea Maurino. ABSTAT: Ontology-Driven Linked Data Summaries with Pattern Minimalization. In ESWC (Satellite Events) 2016, pages 381-395. Springer, 2016.

[215] René Speck, Diego Esteves, Jens Lehmann, and AC Ngonga Ngomo. Defacto-a multilingual fact validation interface. In 14th International Semantic Web Conference (ISWC 2015), 11-15 October 2015, Bethlehem, Pennsylvania, USA (Semantic Web Challenge Proceedings), page 51, 2015.

[216] Richard S Sutton and Andrew G Barto. Reinforcement learning: An introduction, volume 1. MIT press Cambridge, 1998.

[217] Aaron Swartz. Musicbrainz: A semantic web service. IEEE Intelligent Systems, 17(1):76-77, 2002.

[218] Yasufumi Takama and Shunichi Hattori. Mining association rules for adaptive search engine based on rdf technology. IEEE Transactions on Industrial Electronics, 54(2):790-796, 2007.

[219] Jiao Tao, Evren Sirin, Jie Bao, and Deborah L McGuinness. Extending owl with integrity constraints. Description Logics, 573, 2010.

[220] Darya Tarasowa, Christoph Lange, and Sören Auer. Measuring the quality of relational-to-rdf mappings. In International Conference on Knowledge Engineering and the Semantic Web, pages 210-224. Springer, 2015.

[221] Dominik Tomaszuk. Rdf validation: A brief survey. In International Conference: Beyond Databases, Architectures and Structures, pages 344-355. Springer, 2017.

[222] Raphael Troncy and Giuseppe Rizzo. 3cixty: Building Comprehensive Knowledge Bases for City Exploration. Web Semantics: Science, Services and Agents on the World Wide Web, 46-47(Supplement C):2 - 13, 2017. ISSN 1570-8268. doi: https://doi.org/10.1016/j.websem.2017.07.002.

[223] Grigorios Tsoumakas and Ioannis Vlahavas. Random k-labelsets: An ensemble method for multilabel classification. In European conference on machine learning, pages 406-417. Springer, 2007.

[224] Umair Ul Hassan, Amrapali Zaveri, Edgard Marx, Edward Curry, and Jens Lehmann. Acryliq: Leveraging dbpedia for adaptive crowdsourcing in linked 
data quality assessment. In European Knowledge Acquisition Workshop, pages 681-696. Springer, 2016.

[225] Daniel Vila-Suero, Boris Villazón-Terrazas, and Asunción Gómez-Pérez. datos.bne.es: a Library Linked Data Dataset. Semantic Web, 4(3):307-313, 2013.

[226] Boris Villazón-Terrazas, Luis M Vilches-Blázquez, Oscar Corcho, and Asunción Gómez-Pérez. Methodological Guidelines for Publishing Government Linked Data. In Linking government data, pages 27-49. Springer, 2011.

[227] Denny Vrandečić and Markus Krötzsch. Wikidata: a free collaborative knowledgebase. Communications of the ACM, 57(10):78-85, 2014.

[228] Quan Wang, Zhendong Mao, Bin Wang, and Li Guo. Knowledge graph embedding: A survey of approaches and applications. IEEE Transactions on Knowledge and Data Engineering, 29(12):2724-2743, 2017.

[229] Richard Y Wang, Henry B Kon, and Stuart E Madnick. Data quality requirements analysis and modeling. In Proceedings of IEEE 9th International Conference on Data Engineering, pages 670-677. IEEE, 1993.

[230] Zhen Wang, Jianwen Zhang, Jianlin Feng, and Zheng Chen. Knowledge graph embedding by translating on hyperplanes. In Twenty-Eighth AAAI conference on artificial intelligence, 2014.

[231] WEKA. Weka manual for version 3-7-8. Technical report, WEKA, 2013. URL https://pdfs.semanticscholar.org/d617/ d41097bdf97d994d1481adbcfe0c05a51696. pdf.

[232] Kyu-Young Whang, Brad T Vander-Zanden, and Howard M Taylor. A lineartime probabilistic counting algorithm for database applications. ACM Transactions on Database Systems (TODS), 15(2):208-229, 1990.

[233] Longzhi Yang, Daniel Neagu, Mark TD Cronin, Mark Hewitt, Steven J Enoch, Judith C Madden, and Katarzyna Przybylak. Towards a fuzzy expert system on toxicological data quality assessment. Molecular informatics, 32(1):65-78, 2013.

[234] Zhilin Yang, Zihang Dai, Yiming Yang, Jaime Carbonell, Russ R Salakhutdinov, and Quoc V Le. Xlnet: Generalized autoregressive pretraining for language understanding. In Advances in neural information processing systems, pages 5754-5764, 2019. 
[235] A. Zaveri, A. Rula, A. Maurino, R. Pietrobon, J. Lehmann, and S. Auer. Quality Assessment for Linked Data: A Survey. Semantic Web - Interoperability, Usability, Applicability, 2014.

[236] Amrapali Zaveri, Dimitris Kontokostas, Mohamed A Sherif, Lorenz Bühmann, Mohamed Morsey, Sören Auer, and Jens Lehmann. User-driven quality evaluation of DBpedia. In Proceedings of the 9th International Conference on Semantic Systems, pages 97-104. ACM, 2013.

[237] Amrapali Zaveri, Andrea Maurino, and Laure-Berti Equille. Web Data Quality: Current State and New Challenges. International Journal on Semantic Web and Information Systems (IJSWIS), 10(2):1-6, 2014.

[238] Amrapali Zaveri, Anisa Rula, Andrea Maurino, Ricardo Pietrobon, Jens Lehmann, and Sören Auer. Quality assessment for linked data: A survey. Semantic Web, 7(1):63-93, 2015.

[239] Min-Ling Zhang and Zhi-Hua Zhou. Multilabel neural networks with applications to functional genomics and text categorization. IEEE transactions on Knowledge and Data Engineering, 18(10):1338-1351, 2006.

[240] Jun Zhao, Graham Klyne, Matthew Gamble, and Carole A Goble. A checklistbased approach for quality assessment of scientific information. In LISC@ ISWC, pages 34-45, 2013.

[241] Qiankun Zhao and Sourav S Bhowmick. Sequential pattern mining: A survey. ITechnical Report CAIS Nayang Technological University Singapore, 1:26, 2003. 